\title{
Butterflies as Signal Receivers
}

A Thesis submitted for the degree of

Doctor of Philosophy

by

Alexandra L.J. Freeman

Linacre College

Oxford University

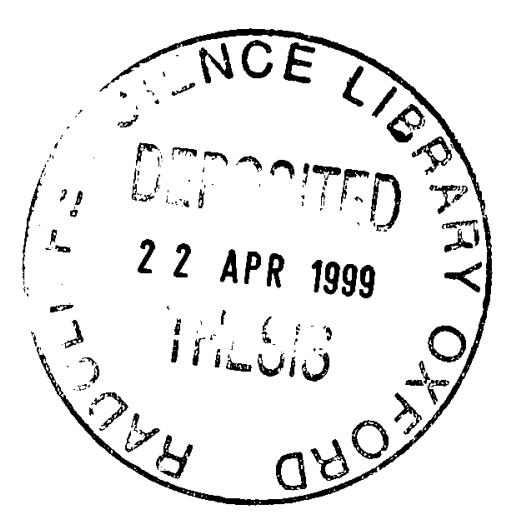

Michaelmas Term 1998 


\section{Butterflies as Signal Receivers}

\section{Abstract}

This thesis examines the existence of colour preferences in butterflies. Two polymorphic species - the Mocker Swallowtail (Papilio dardanus) and the Silver-Washed Fritillary (Argynnis paphia) are used as study species. Both the basis of colour preferences in mate choice and flower choice during feeding, and the implications of the preferences for the evolution of the species and morphs are investigated.

In the Silver Washed Fritillary a non-genetically determined preference exists for highly saturated orange coloration in both mate and flower choice. This is shown not to be due to a bias for orange in colour reception through the use of electroretinograms, measuring the electrical output of the retina when exposed to light of varying wavelengths. It has not been possible, however, to rule out the possibility that the preference for the most common, orange, female morph is learnt.

The flower colour preferences of the Mocker Swallowtail are investigated, and it is demonstrated that individuals show an initial preference for blue flowers, and also learn rapidly to feed off flowers of other colours that prove profitable. Their decision to try flowers of other colours is shown to be influenced by the behaviour of other individuals. In previous mate choice experiments, experienced males of the species have been shown to have a preference for the most common, black and white, morph. The colours of the morphs and the spectral sensitivities of the butterflies are analysed quantitatively. The initial and subsequent preferences of naive males are investigated in behavioural experiments, and a possible influence of learning on their subsequent choices is discovered. No influence of female choice is found. This information is then used to create a mathematical model of the population structure, for which it is also necessary to determine the relative palatabilities of the model and mimic, and the mating frequency of wild males. The model demonstrates how the observed population structures might arise through evolutionary time.

Measurements of the morphology of males and females of Papilio dardanus, and one of its putative models, Danaus chrysippus, shows that in Papilio dardanus females the centre of gravity is positioned significantly further back than in males and in Danaus chrysippus. This positioning far from the wing base has already been shown to handicap an individual escaping from a predator due to decreased aerobatic ability. It has also already been shown that mimetic species tend to have centres of mass positioned further back than non-mimetic species, and hence it is possible that the position of a centre of mass of a butterfly (and its effect on agility) may be a factor in the evolution of mimicry in a species or (where females carry a large egg load) in females of a species only. 


\section{Acknowledgements}

The work in this thesis would not have been possible without help from a great many people. I have included acknowledgements at the end of each chapter to thank those people who gave specific help, but there are many people who I would like to thank additionally for their help and support throughout the time I have been working on this project.

I would firstly like to thank my supervisor, Tim Guilford, for taking me on as a student, helping me design the project, and for all his help throughout the years I have been working on it. I am particularly grateful for his advice on writing up.

Without the financial assistance of the my aunt Molly Jewell - who will sadly never see this thesis - and the additional support of my parents I might never have undertaken to do this work, and I would especially like to thank my father for proof-reading the entire thesis.

I would also like to thank Sir Cyril Clarke, Alison Gill and Dick Vane Wright for all their encouragement and for sharing their mine of knowledge on Papilio dardanus, and especially for the loan of brood books. I am deeply indebted to Yan Wong for explaining statistics to me, and correcting my misuse of statistical tests! I would not have been able to get hold of butterflies without the help of many people in the Entomological Livestock Group, and particularly John Calvert at Stratford Butterfly Farm. I have also been kept (relatively) sane with the help of many friends - members of the ABRG, Linacre, the Arcadians, Merton Choir, and New College Development Office.

Finally I would like to thank especially John Alden, who has put up with me and the butterflies for over three years, and without whom this thesis would have been immeasurably worse. 


\section{Butterflies as Signal Receivers}

\section{Table of Contents}

\section{Acknowledgements}

Chapter 1 - Introduction $\quad 1$

Outline 2

Mimicry - The eye of the beholder 2

Perception and bias $\quad 4$

$\begin{array}{ll}\text { Learning } & 10\end{array}$

The study of Papilio dardanus 11

$\begin{array}{ll}\text { References } & 12\end{array}$

Chapter 2 - Mate choice and colour preferences in Argynnis paphia 15

$\begin{array}{ll}\text { Introduction } & 16\end{array}$

$\begin{array}{ll}\text { Aim } & 18\end{array}$

$\begin{array}{ll}\text { Behavioural observations } & 19\end{array}$

Experiment 2-1 Male mate preference: Male versus paphia female
Introduction

$\begin{array}{ll}\text { Aim } & 20\end{array}$

$\begin{array}{ll}\text { Method } & 20\end{array}$

Results $\quad 21$

Discussion $\quad 22$

Experiment 2-2 Male mate preference: Paphia versus valesina females
Introduction

$\begin{array}{ll}\text { Aim } & 24 \\ & 24\end{array}$

$\begin{array}{ll}\text { Method } & 24\end{array}$

Results $\quad 24$

Discussion $\quad 26$

Experiment 2-3 Reflectance spectra of Argynnis paphia

$\begin{array}{ll}\text { Introduction } & 27 \\ \text { Aim } & 27\end{array}$

Aim

$\begin{array}{ll}\text { Method } & 27 \\ \text { Results } & 28\end{array}$

Results

Experiment 2-4 Electroretinogram of Argynnis paphia

Introduction $\quad 30$

Aim $\quad 32$

Method $\quad 32$

Results $\quad 34$

Discussion $\quad 35$

Conclusions $\quad 36$

$\begin{array}{ll}\text { Acknowledgements } & 37\end{array}$

$\begin{array}{ll}\text { References } & 37\end{array}$

Chapter 3 - Analysis of the visual signals in Papilio dardanus 39

$\begin{array}{ll}\text { Introduction } & 40\end{array}$

Aims $\quad 42$

Experiment 3-1 Photography under UltraViolet light
Introduction

Aim 44 
Method \& Materials $\quad 45$

Results $\quad 46$

$\begin{array}{ll}\text { Discussion } & 49\end{array}$

Experiment 3-2 Analysis of the pigments using a Fluorescence Spectrometer Introduction $\quad 50$

Aim 51

Method \& Materials $\quad 51$

Results $\quad 52$

Discussion $\quad 54$

Experiment 3-3 Reflectance spectra of the pigments
Introduction

Aim $\quad 55$

Method \& Materials $\quad 55$

$\begin{array}{ll}\text { Results } & 57\end{array}$

$\begin{array}{ll}\text { Discussion } & 63\end{array}$

Experiment 3-4 Scanning Electron Microscopy of butterfly scales
Introduction

$\begin{array}{ll}\text { Aim } & 67\end{array}$

$\begin{array}{ll}\text { Method \& Materials } & 67\end{array}$

$\begin{array}{ll}\text { Results } & 68\end{array}$

Discussion $\quad 71$

Experiment 3-5 Transmission Electron Microscopy of butterfly scales
Introduction

Aim $\quad 72$

Method \& Materials $\quad 72$

$\begin{array}{ll}\text { Results } & 72\end{array}$

Discussion $\quad 77$

Experiment 3-6 Electroretinogram of Papilio dardanus
Introduction

$\begin{array}{ll}\text { Aim } & 79\end{array}$

$\begin{array}{lr}\text { Method \& Materials } & 80\end{array}$

$\begin{array}{ll}\text { Results } & 81\end{array}$

Discussion $\quad 84$

$\begin{array}{cr}\text { Experiment 3-7 Fading of Papiliochrome II } & 85 \\ \text { Introduction } & \end{array}$

Aim $\quad 86$

$\begin{array}{ll}\text { Method \& Materials } & 86\end{array}$

$\begin{array}{ll}\text { Results } & 87\end{array}$

$\begin{array}{ll}\text { Discussion } & 89\end{array}$

$\begin{array}{lr}\text { Conclusions } & 90\end{array}$

Acknowledgements $\quad$. $\quad 92$

$\begin{array}{ll}\text { References } & 93\end{array}$

Chapter 4 - Flower choice in Papilio dardanus $\quad 97$

\begin{tabular}{lr} 
Introduction & 98 \\
\hline
\end{tabular}

Aims $\quad 99$

Experiment 4-1 Flower colour preference and constancy in Papilio dardanus

$\begin{array}{ll}\text { Introduction } & 100\end{array}$

$\begin{array}{ll}\text { Aim } & 100\end{array}$

$\begin{array}{ll}\text { Method \& Materials } & 100\end{array}$

Results 101

$\begin{array}{ll}\text { Discussion } & 104\end{array}$

Experiment 4-2 Investigation of the effect of painted vs unpainted flowers on preference

$\begin{array}{ll}\text { Introduction } & 106\end{array}$

$\begin{array}{ll}\text { Aim } & 106\end{array}$

$\begin{array}{ll}\text { Method \& Materials } & 106\end{array}$ 
Results

Discussion

Experiment 4-3 The effect of matt/gloss finish and colour of flower centre on preference

Introduction

Aim

Method \& Materials

Results

Discussion

Experiment 4-4 The effect of another individual feeding on choice of colour Introduction

Aim

Method \& Materials

Results

Discussion

Experiment 4-5 Effect of another individual on flower choice Introduction

Aim

Method \& Materials

Results

Discussion

Conclusions

Chapter 5 - Mate choice in Papilio dardanus $\quad 124$

\begin{tabular}{ll} 
Introduction & 125 \\
\hline & 128
\end{tabular}

Aims

Behavioural Observations

Experiment 5-1 Initial morph preference of male Papilio dardanus Introduction

Aim

Method \& Materials

Results

Discussion

Experiment 5-2 Second morph choice of male Papilio dardanus

Introduction

Aim

Method \& Materials

Results

Discussion

Experiment 5-3 Genetics of males showing morph preferences

Introduction

Aim

Method \& Materials

Results

Discussion

Experiment 5-4 Female preferences for symmetry and swallowtails

Introduction

Method \& Materials

Results

Discussion 
Chapter 6 - The palatability of Papilio dardanus

Introduction

Aim

Experiment 6-1 Pilot test to determine the suitability of hen chicks for palatability experiments

Introduction

Aim

Method \& Materials

Results

Discussion

Experiment 6-2 Pilot test to determine the suitability of starlings (Sturnus vulgaris) for palatability experiments

Introduction

Aim

Method \& Materials

Results

Discussion

Experiment 6-3 A discrimination test using dried butterflies and starlings (Sturnus vulgaris)

Introduction

Aim

Method \& Materials

Results

Discussion

Experiment 6-4 A further discrimination test using dried butterflies and starlings (Sturnus vulgaris)

Introduction

Aim

Method \& Materials

Results

Discussion

Experiment 6-5 A discrimination test using fresh butterflies and starlings

(Sturnus vulgaris)

Introduction

Aim

Method \& Materials

Results

Discussion

Experiment 6-6 Comparison of the morphology of Papilio dardanus and its models

Introduction

Aim

Method \& Materials

Results

Discussion

Conclusion

Aim

Method

Factor 1 Survival without predation, fecundity, and survival of offspring Introduction 
Discussion

Factor 2 Mate choice

Introduction

Calculations

Results

Factor 3 Predation

Introduction

Method

Results

The mathematical model

Introduction

Method

Results

Discussion

Conclusions

Appendix 1 - The races and morphs of Papilio dardanus

Appendix 2 - Electroretinogram calibration

ix

Appendix 3 - The flight cage used in behavioural experiments

$\mathrm{xviii}$

Appendix 4 - Analysis of data from Ford (1936)

xix

Appendix 5 - Analysis of data from Cook et al. (1994)

xxvii

Appendix 6 - Program listing

xxxii 


\section{Chapter One}

\section{Introduction}

"As the laws of Nature must be the same for all beings, the conclusions furnished by this group of insects must be applicable to the whole organic world; therefore, the study of butterflies - creatures selected as the types of airiness and frivolity - instead of being despised, will some day be valued as one of the most important branches of Biological science."

H.W. Bates (1864) 


\section{Outline}

In this thesis I explore some of the most interesting aspects of biology - natural selection and mimicry; perceptual biases, learning and sexual selection leading to speciation. I investigate all these factors by studying and carrying out experiments on polymorphic butterfly species. One is the wellknown species Papilio dardanus (the Mocker Swallowtail), which has many geographic races in Africa, each with a different balance of mimetic and non-mimetic morphs. The other is Argynnis paphia (the Silver-Washed Fritillary), which has two morphs, the proportions of which vary across its range. The genetic control of the morphs in both species is quite well understood, and preliminary behavioural experiments have been carried out indicating that males show morph preferences in their choice of mates.

In this chapter, I introduce the background to each aspect of the thesis. Chapter 2 is an investigation of the mate colour preferences of Argynnis paphia, attempting to clarify the basis of the preferences previously found for bright orange flowers and mates. In Chapter 3, which introduces and provides background material on Papilio dardanus for the subsequent chapters, I analyse the colours used by the species and its models, and also the visual sensitivities of the butterflies, in order to gain an idea of how the butterflies may appear to each other and to their predators. In Chapter $4 \mathrm{I}$ determine the feeding colour preferences of Papilio dardanus, and also investigate the role of learning and social influences on their flower choice. Chapter 5 concerns the mate preferences of Papilio dardanus, following on from the fieldwork of Cook et al. (1994) in which wild males were shown to have a preference for black and white females over other morphs. I investigate the basis for this preference, including the role of learning. In Chapter 6 I describe work on the palatability of Papilio dardanus in comparison with its models. Finally, in Chapter 7, I construct a mathematical model of the balance of morphs in a population, taking into account the results of the previous chapters, to demonstrate how the balanced polymorphism may be maintained.

Each chapter is written to stand on its own, and includes a short introduction to the literature surrounding each topic. I have not attempted to review the literature exhaustively where good review articles already exist, but have recommended the reader to articles which do this where possible.

\section{Mimicry - the eve of the beholder}

The subject of mimicry has long been a matter of great debate among biologists. H. W. Bates formally introduced the concept of mimicry in 1861 (only two years after the publication of Darwin's 'Origin of Species') in a reading at the Linnean Society of London after 11 years studying butterflies in the Amazon. It has still to be satisfactorily defined. 
The problem with the definition of mimicry is the range of phenomena which it can include. One of the most controversial issues is the position of crypsis. Vane-Wright probably came closest to a satisfactory definition in 1980 when he said:

"Mimicry involves an organism (the mimic) which simulates signal properties of a second living organism (the model) which are perceived as signals of interest by a third living organism (the operator), such that the mimic gains fitness as a result of the operator identifying it as an example of the model."

In this definition, Vane-Wright demonstrates an important principle - that the message of the signal is determined by the psychology of the signal receiver (or 'operator' to use Vane-Wright's terminology). This view point makes a distinction between crypsis and mimicry since in crypsis an organism is trying to avoid the transmission of signals to a receiver who may be a predator whilst a mimetic organism is actively attempting to signal to any potential predator receivers (Silberglied, 1977). Different receivers, however, have different states-of-mind which affect the perceived information in a signal. This means that it is impossible to classify a species with regard to its mimicry or crypsis without taking into account the potential signal receivers. For example, Rothschild (1981) describes how burnet moths (Zygaena sp.), with their classic black and red warning colours, can easily be overlooked as seed pods of the nectar plant Vicia cracca. An entomologist can suddenly see individual moths which they had previously seen as seed pods from further away, and she also describes how, as the entomologist gets older, the moths are more frequently overlooked as pods. This demonstrates how small differences in the signal receiver's perception can alter the classification of a signal. This is an important theme in this thesis as I explore how butterflies receive and perceive various signals (specifically with regard to the role of colour in food and mate choice).

Mimicry is classically divided into two types: Batesian mimicry, in which the mimic is palatable; and Mullerian mimicry, in which the mimic is unpalatable. However, there is naturally a 'palatability spectrum' (Brower et al., 1968) which means that there is a considerable grey area in which species cannot be classified as either Batesian or Mullerian mimics, the extremes of the spectrum. Palatability varies between individuals of a species, often depending upon their foodplant (Brower et al., 1968), and the palatability also depends on the predator concerned. A good example of variable palatability is described again by Rothschild (1981): cuckoos (Cuculus canorus) easily recognise black-and-yellow hypsid caterpillars and similar mimics, but whilst as young fledglings they assiduously avoid them (as they prove highly indigestible and harmful to the young birds), as adults they actively seek them out and show no ill-effects of eating even very large numbers of them. 
Batesian and Mullerian mimics have different population dynamics. If the mimic is palatable, then the model's own protection is endangered. This means that the mimics must always be rare with respect to the models as the risk to them is positively density-dependent (as their density with respect to their model increases, so does the attack rate on them as predators find more of the similar-looking individuals palatable). If the mimic is as unpalatable as the model then they both gain equally from the similarity. The risk to unpalatable, aposematic morphs or species is negatively density-dependent (as their density decreases, the frequency of attacks on them increases as predators are less likely to recognise the signal of unpalatability). This difference theoretically allows a distinction between the two systems to be made in the field, and this has been tested by Benson (1972) who captured individuals from a natural population of the butterfly Heliconius erato and altered their patterns in one of two ways. Recapture data showed that the rarer of the two altered patterns had a lower survival rate, suggesting that the species was a Mullerian mimic. Gordon (1987) showed by standard capture/re-capture that the commonest forms of Hypolimnas misippus had a lower survival rate than the rarer forms, and other evidence showed that this was likely to be due to increased predation, suggesting that its mimicry is Batesian.

However, since a species may differ in palatability to different predators or across its range (due to changes in foodplants, which often provide the toxic chemicals which make a butterfly unpalatable), just as the signals an individual gives out may have different meanings to different individual predators, and so it may not be possible to classify a species' mimicry in such general terms. One of the species studied in this thesis, Papilio dardanus is often cited as a classic example of a polymorphic Batesian mimic. However, there is a little evidence to suggest that this may not be entirely true, and that the species may possess some chemical protection. This is the subject of Chapter 6.

\section{Perception and bias}

Mimicry is only one example of signalling, and the importance of signal receiver psychology holds for all signals. Throughout the natural world, aposematic coloration has converged on bold red and yellow (sometimes white) markings, often with black. These colours are very obvious against a wide range of backgrounds, and appear to exploit the senses of predators to achieve universal conspicuousness. The exact reason why defended prey are almost always conspicuous is debated, but they appear to be exploiting the psychology of the predators in some way to decrease the chance of attack (which would not be beneficial to either predator or prey). It may be that the colours are more readily associated with unpalatability (Rothschild, 1984), that the conspicuous colours are more easily remembered (Gittleman \& Harvey, 1980; Turner, 1984), that the novel pattern will be easier to form an association of unpalatability with (Turner, 1975), or that the conspicuousness reduces accidental 
attacks by increasing the 'detection distance' (Guilford, 1986). Other hypotheses are reviewed in Guilford, 1990. In fact, as in most natural systems, it is likely that many factors are acting at once, different ones being more important with different predator species and individuals. In each case, however, it is the way in which the predator perceives and interprets the environment which determines the signals used by the prey.

It is not just the psychology of animals which affects the meaning of a signal. The perceptual systems themselves can have 'inherent bias' (Arak \& Enquist, 1993) for certain colours and patterns. If a bias exists, it is easy to see how this is likely to be 'exploited' by a signal. Such biases could be very important in initiating a signal - which may subsequently become very important in sexual selection, being subject to Fisherian run-away selection, or, as recently suggested, acting as a means of driving sympatric speciation (Turner and Burrows, 1995). Arak and Enquist refer to these biases for forms which may not actually exist in nature as 'hidden preferences', and describe how these allow for the evolution of novel forms of stimulus which may prove even more striking to the receiver than the existing forms to which it reacts. They used simple artificial neural networks to demonstrate this phenomenon, although the method that they used was unrealistic and could not readily be applied to living systems. Such simple neural network designs have been criticised, for example by Dawkins and Guilford (1995), for being unrealistic in their interpretation.

There are many ways in which the whole area of using artificial neural networks can be misleading. Most of the papers claiming to simulate, in some way, a biological property of perceptual systems (e.g. Arak \& Enquist, 1993; Enquist \& Arak, 1993; Johnstone, 1994) use an input layer ('artificial retina'), a single hidden layer, and a single cell output layer neural network. They present the input layer with categorical data (each cell has the value of either 1 or 0 ), and train the network using only a few stimuli (either a positive or negative). They then present the network with numerous other stimuli and assess the response of the network to each. Artificial neural networks are not designed for this sort of task. They do not simulate neurones in any meaningful way, and are merely a means of non-linear regression on continuous data (fitting $a^{*}$ curved surface to a multi-dimensional dataset). In this case, the dataset may be seen as in multi-dimensional space (one dimension for each input), but the points being used are at the corners of a hypercube (since the input can be either 0 or 1 , but never anywhere along the continuum between these extremes). The neural network is only trained to interpolate from two data points (the two training stimuli), and is then asked to predict the values at corners of the hypercube (by presenting it with other datapoints at corners and recording the response). This explanation of the task presented demonstrates how misleading the results can be. The 'hidden preferences' which may appear to have arisen in the network are caused by the fact that the task is to regress a complex surface on the basis of only a few data points. However, the theory of 'hidden preferences' could be demonstrated by using artificial neural networks - the rest of the 
hypersurface which is untrained could represent the area of possible stimuli which is not under selection.

Apart from these fundamental problems with the experimental design, particular flaws in some cases have lead to false conclusions being drawn from the results. Enquist and Arak (1993) attempted to use an artificial neural network model to illustrate the evolution of exaggerated male traits (in this case long tails). They used a $6 \times 6$ node input layer, each node being connected to each of 10 hidden cells, which in turn were all connected to an output cell. It is therefore apparent that the way in which the 36 input cells are arranged (and thus the apparent 'pattern' projected onto them) is irrelevant. For the network, the 'pattern' is simply an array of 36 binary numbers (Enquist and Arak rather misleadingly describe this as 'a network representing the recognition system of a female bird'). The network is then trained in 'a procedure that mimics the process of natural selection of recognition systems over evolutionary time'. This is done by starting with a random series of weightings on all the connections, recording the output value (which is normalised between 0 and 1 ), and then 'mutating' a tenth of the weightings, and comparing the output value. The better network was retained and the process repeated. This was done until the probability of the network producing an output value of less than 0.5 for a 'correct' stimulus was less than $10^{-5}$. The result of such a 'threshold' in the training procedure means that there is no pressure on the network producing an output of 0.6 to a 'correct' stimulus to improve on this (and vice versa). Hence the training is weak, and the results at the end of the training period indicate this (response to 'correct' stimulus 0.60 and 0.61 , to 'incorrect' stimulus 0.42 and 0.41 for networks I and II respectively). In addition the 'incorrect' stimulus has 5 ' 1 ' inputs (out of the total of 36 ), whilst the 'correct' stimulus has 6 . Thus it only takes a majority of the weightings in the network to become positive for the network to become, effectively, a kind of counting machine - the greater the number of ' 1 ' inputs the greater the output, and given the weak training this could easily become selected for (as the output is only judged on whether or not it is greater than 0.5 ).

Enquist and Arak only published five of the test images together with the responses of their two networks. Four gave 'positive' responses, and these had 7,8,8, and 9 ' 1 ' inputs (two of them are shaped to represent increasingly long tails, and one is a shape which does not at all resemble the training pattern). One gave a 'negative' response, and had only 4 ' 1 ' inputs. Enquist and Arak interpret this as a demonstration of 'peak shift' behaviour (where a slight super-stimulus gives an exaggerated positive result and vice versa), claiming that "this proves that the recognition mechanism itself exerts selection pressure on the signal; in this case there is a 'bias' in the mechanism favouring males with longer tails". In fact it is entirely possible that the results they obtained were due to the network giving a greater output with an increased input (due to an increased number of positive weightings translating the increased number of positive inputs into a greater output value). 
The same training procedure was employed by Arak \& Enquist (1993) in which they attempted to illustrate the presence of two hidden preferences in two different neural networks representing "the recognition system of a hypothetical insect species faced with the problem of discriminating between flowers which differ in profitability". The networks in this case were trained with only two stimuli:

\begin{tabular}{|c|c|c|c|c|c|c|c|c|c|c|c|c|c|c|c|c|c|c|c|c|c|c|c|c|c|c|c|c|c|}
\hline Node & 1 & 2 & 3 & 4 & 5 & 6 & 7 & 8 & 9 & & & & & & 15 & & & 18 & & & & & & & & 26 & & & $29 \mid 30$ \\
\hline Correct & 0 & $\underline{0}$ & 1 & 0 & $\underline{0}$ & 0 & 1 & 0 & 1 & 0 & $\underline{0}$ & 0 & 1 & $\underline{0}$ & 0 & $\underline{0}$ & $\underline{0}$ & 1 & 0 & $\underline{0}$ & $\underline{0}$ & 0 & $\underline{0}$ & 0 & $\underline{0}$ & 0 & $\underline{0}$ & 0 & 0 \\
\hline Incorrect & 0 & 0 & 1 & 0 & 0 & 0 & 1 & 1 & 1 & 0 & 0 & 0 & 1 & 0 & 0 & 0 & 0 & 0 & 0 & 0 & 0 & 0 & 0 & 0 & 0 & 0 & 0 & 0 & 0 \\
\hline
\end{tabular}

It is obvious that the only differences between these two arrays are nodes 8 and 18 , and that the networks will therefore be trained to discriminate between the two arrays on the basis of these two nodes. Arak and Enquist discovered that two of their networks (in saying that "many other networks exist that can achieve the task just as well" they hint that these two were chosen from a greater number in order to illustrate their point) appeared to discriminate between the arrays in different ways - one on the basis of the status of node 8 and one on that of node 18. However, this result itself demonstrates the ineffectiveness of the training routine. Again, the threshold method was used (at the end of training the two networks gave output values of 0.61 and 0.62 for the 'correct' stimulus and 0.41 and 0.38 for the 'incorrect' stimulus), and this has resulted in the two networks becoming stuck at local maxima in their response, rather than finding the global maximum response (which would be to use the status of both nodes 8 and 18 as a discrimination mechanism).

Other training mechanisms, which use genetic algorithms (ranking a population of networks relative to each other, selecting at each generation from those which had the highest outputs to a 'correct' stimulus combined with the lowest outputs to an 'incorrect' stimulus, and using 'mutations' to move from local maxima) are much more stringent, and will eventually converge on a global maximum. The fact that two of Arak and Enquists networks show very different responses only serves to highlight the fact that they have not converged on the best discrimination mechanism, and therefore that the training has not been successful. The networks are only performing a mathematical curve-fitting procedure through the data and hence there should be a 'best-fit' which all networks would converge upon when correctly trained. Arak and Enquist conclude "Only if the network is initially trained using all possible input patterns as exemplars can a perfect recognition system evolve". Although this conclusion (illustrating how perceptual biases may arise in natural recognition systems) may be correct, the method they have employed to illustrate it seems weak. The choice of such a threshold training mechanism does not even simulate natural selection processes in any way. Natural selection is a continuous process, resulting from competition between individuals and not related to the ability to reach a threshold, or stopping at that point. 
By contrast, Johnstone (1994) used a similar artificial neural network structure ( 4 x 5 inputs, 5 hidden cells), but a different training method to illustrate female preference for symmetrical traits. He trained five networks (again "representing the recognition system of a female bird") to a set of either 4 or 5 patterns, mutating a twentieth of the connections each time. However, after the mutation each time he chose only one network from the population for 'replication', and he ranked them by "determining the mean value of its response to the patterns in the training set, and the mean value of its response to a set of randomly generated patterns, and subtracting the latter value from the former". The network with the highest score was then chosen to be mutated to form a new population of five competing networks. This was repeated 50 times. This training procedure appears to have been very successful since his results showed a clear pattern in preference emerging after training in 1000 networks which must have converged on the same 'result'.

His five training patterns were:

\begin{tabular}{|l|c|c|c|c|c|c|c|c|c|c|c|c|c|c|c|c|c|c|c|c|}
\hline Node & 1 & 2 & 3 & 4 & 5 & 6 & 7 & 8 & 9 & 10 & 11 & 12 & 13 & 14 & 15 & 16 & 17 & 18 & 19 & 20 \\
\hline Pattern 1 & 0 & $\mathbf{1}$ & $\mathbf{1}$ & 0 & 0 & $\mathbf{1}$ & 0 & 0 & 0 & $\mathbf{1}$ & 0 & 0 & 0 & $\mathbf{1}$ & 0 & 0 & 0 & $\mathbf{1}$ & 0 & 0 \\
\hline Pattern 2 & 0 & $\mathbf{1}$ & $\mathbf{1}$ & 0 & 0 & $\mathbf{1}$ & $\mathbf{1}$ & 0 & 0 & $\mathbf{1}$ & 0 & 0 & 0 & $\mathbf{1}$ & 0 & 0 & 0 & 0 & 0 & 0 \\
\hline Pattern 3 & 0 & $\mathbf{1}$ & $\mathbf{1}$ & 0 & 0 & $\mathbf{1}$ & $\mathbf{1}$ & 0 & 0 & $\mathbf{1}$ & $\mathbf{1}$ & 0 & 0 & 0 & 0 & 0 & 0 & 0 & 0 & 0 \\
\hline Pattern 4 & 0 & $\mathbf{1}$ & $\mathbf{1}$ & 0 & 0 & $\mathbf{1}$ & $\mathbf{1}$ & 0 & 0 & 0 & $\mathbf{1}$ & 0 & 0 & 0 & $\mathbf{1}$ & 0 & 0 & 0 & 0 & 0 \\
\hline Pattern 5 & 0 & $\mathbf{1}$ & $\mathbf{1}$ & 0 & 0 & 0 & $\mathbf{1}$ & 0 & 0 & 0 & $\mathbf{1}$ & 0 & 0 & 0 & $\mathbf{1}$ & 0 & 0 & 0 & $\mathbf{1}$ & 0 \\
\hline
\end{tabular}

Each had 6 ' 1 ' inputs, and it can be seen that the informative nodes are $6 \& 7,10 \& 11,14 \&$ 15 , and $18 \& 19$. Johnstone found that networks trained to respond to all 5 patterns, and those which were only trained on patterns $1,2,4$ and 5 , all showed a preference for pattern 3 , followed by patterns 2 $\& 4$, and then by patterns $1 \& 5$. This is probably the result of the networks converging on the same system of recognition. At the crucial nodes, the networks are using the best guess they can at the value. At nodes $6 \& 7$ three of the four training sets (ignoring 3) have a ' 1 ', so the networks are looking for a ' 1 ' here, at $18 \& 19$ three of the four have a ' 0 ' so a ' 0 ' is the best indicator of a positive stimulus. At $10 \& 11$ and $14 \& 15$ the four stimuli are half ' 1 ' and half ' 0 ', so without pattern 3 the network might be expected to ignore these nodes as uninformative. When tested on other patterns, the networks trained on all 5 patterns showed an even greater response to another pattern: 


\begin{tabular}{|l|c|c|c|c|c|c|c|c|c|c|c|c|c|c|c|c|c|c|c|c|}
\hline Node & 1 & 2 & 3 & 4 & 5 & 6 & 7 & 8 & 9 & 10 & 11 & 12 & 13 & 14 & 15 & 16 & 17 & 18 & 19 & 20 \\
\hline Pattern & 0 & $\mathbf{1}$ & $\mathbf{1}$ & 0 & 0 & $\mathbf{1}$ & $\mathbf{1}$ & 0 & 0 & $\mathbf{1}$ & $\mathbf{1}$ & 0 & 0 & $\mathbf{1}$ & $\mathbf{1}$ & 0 & 0 & 0 & 0 & 0 \\
\hline
\end{tabular}

Johnstone explained this preference as being caused by the untrained pattern overlapping more of the training set average. In real terms, more networks are using the information of a ' 1 ' in nodes 14 and 15 as indicators of a positive stimulus than might be expected given that $60 \%$ of the time the positive stimuli have a ' 0 ' in these positions. Perhaps this is due to the negative stimuli presented during training. Whatever the reason, Johnstone's statement that "the above results thus indicate that when males of a species possess paired ornaments, selection for female recognition of appropriate mates can lead to biases favouring symmetrical males" appears to be rather an unsubstantiated claim. There is no evidence to suggest that behaviour illustrated by artificial neural networks closely models that shown in real systems.

Despite these criticisms of the methods used to demonstrate the possible existence of 'perceptual biases' and 'hidden preferences' in natural recognition systems, the theory remains sound. Recently, 'hidden preferences' have been invoked as a possible cause of the dramatic speciation of the cichlid fish in African lakes (Galis and Metz, 1998), and some cases of biases appear to have been shown experimentally. Basolo $(1990,1991,1995)$ demonstrated a preference for swords in swordless platyfish (Xiophophorus maculatus and Xiophophorus variatus), whose genus also includes swordfish who possess such a sword. Phylogenies of the genus disagree as to whether the presence of a sword (and the preference for it) are a primitive characteristic, with the sword subsequently being lost by two platyfish clades; or whether the preference for swords is a pre-existing primitive condition, and that the two swordfish clades have subsequently evolved swords. Ryan \& Rand (1993) showed the existence of perceptual biases in Tungara frogs (Physalaemus spp.), where, when the 'chuck' portion of the male call of Physalaemus pustulosus (which stimulated one of the female inner ear receptors) was added to the call of other species, such as Physalaemus coloradorum, the latter was rendered more attractive to conspecific females. Thus it appears that Physalaemus females have a perceptual bias for the 'chuck' which has been exploited by male Physalaemus pustulosus, but which has not yet evolved in other species.

Another example of this seemed to be possible when Cook et al. (1994) described a preference in male Papilio dardanus (the African Mocker Swallowtail) for the black and white female morph over the black and yellow morph and the black, orange and yellow/white morph. Given that males show this preference, and yet only one part of the range of one race (the Western area of the range of race dardanus) can be considered to have gone to fixation, with all females being of the black and white (hippocoonides) morph, it must be assumed that the other morphs have some advantage so as to keep the population in a balanced polymorphism. Since the hippocoonides morph is considered to be 
a Batesian mimic, it could be positive frequency-dependent predator pressure which keeps this balance. Whatever the advantage to the other morphs, given that the polymorphism is balanced the males should theoretically have no morph preference. It appears, therefore, that the hippocoonides females are intrinsically more attractive to the wild males for some reason. I investigate the basis of this in Chapters $3 \& 5$.

\section{Learning}

The psychology of the receiver can be altered in many ways, all of which can affect how signals are perceived and interpreted. For example, the physiological state of the receiver, such as how hungry it is, may make some signals appear to be more obvious or demanding than others - a starving animal may not recognise a predator when it can also see food. One of the most important ways in which a receiver's perception can change is through learning. Predators learn to avoid aposematic animals and form a 'search image' for their prey. Butterflies have been shown to learn to recognise rewarding flowers (see Chapter 4 , Introduction). The ability to learn allows a much greater plasticity in response from receivers. Although flower preferences can be innate, the ever-changing range of flowers available to nectar-feeding animals means that a degree of flexibility in preference is highly advantageous. Such an ability may extend to the recognition of individual characteristics, and allow individual recognition in a species. This can be highly advantageous in species where the same individuals may be encountered again (such as in territorial and social animals) as it gives the receiver further, background information about the content of the signal and allows a more sophisticated interpretation of it. I investigate the role of learning in flower choice in Papilio dardanus in Chapter 4.

Returning to the case of Papilio dardanus and mate choice, the preferred hippocoonides morph is also the most common in the race studied. In a polymorphic species it may be advantageous for individuals to learn to recognise the patterns of potential mates so that they can avoid wasting resources chasing butterflies of the wrong species or sex. If this were the case, then a male may learn to recognise the pattern of his first mate and subsequently choose females of the same morph more frequently. Since hippocoonides is the most common morph, experienced males are most likely to have come across this morph as their first mate, and thus have acquired a preference for hippocoonides in their subsequent matings. I investigate the possibility of learning being involved in mate choice in Papilio dardanus and also in the British species Argynnis paphia (the Silver-washed Fritillary) which has two female morphs and in which there is a similar preference for the more common morph (Magnus, 1958). 


\section{The study of Papilio dardanus}

Papilio dardanus (Brown), the Mocker Swallowtail, is a classic example of a polymorphic and mimetic butterfly. It is found in about 13 geographically distinct races in sub-Saharan Africa, including Madagascar and the Comoro Islands, and throughout its range the males are yellow and black with characteristic 'swallowtails' on the hind wings, with only slight variations in the degree of black patterning. The females, however, are polymorphic, and display many morphs in differing proportions according to the geographical race. Many of these morphs are mimetic, and their distribution corresponds to the distribution of their model. See Appendix 1 for a summary of the races and morphs.

In the early part of the century there was great debate as to how mimicry could evolve according to Darwin's theory of natural selection. It was evident that forms intermediate between the original non-mimetic form and the perfect mimic would have no selective advantage. It also became evident that crosses between mimetic forms never appeared to produce intermediate forms, making it seem unlikely that there were many genes involved, each with small effects. Biologists such as Punnett (1915) and Goldschmidt (1945) argued that evolution proceeded in large jumps, due to mutation, and that natural selection only acted to perfect the mimicry after a likeness had been achieved by random change. Others (such as Fisher, 1927; Carpenter, 1946; and Ford, 1953) believed that random mutation could not have produced so many mimetic species and forms, and their ideas formed a 'two-phase' theory. According to this, a mutation causing any resemblance (particularly in colour) would spread in a population since predators often generalise, and even a slight increase in the chance of avoiding predators would be advantageous. Then there would be selection for 'modifier genes' which perfect the mimicry and which are closely linked, thus intermediate forms due to interbreeding of morphs is avoided. An article by Clarke et al. (1995) details the discussion in much greater detail.

With its many races and morphs, Papilio dardanus provided an excellent opportunity for further understanding of the genetics involved in mimicry. In the 1950s Sir Cyril Clarke and Philip Sheppard began to breed Papilio dardanus in Britain, after discovering that the butterflies can be mated easily by hand. Through many years of breeding and meticulous note-taking, they pieced together the genetics of many races, and published their results in a series of four papers $(1959,1960 \mathrm{a}$, 1960b, 1962). By making crosses between races (especially the non-mimetic races of Madagascar and the Comoro Islands) they not only discovered the main genes involved, but also investigated the presence of local modifier genes keeping the mimicry perfect. The system turned out to be relatively simple - the morphs were controlled by 'supergenes', made up of closely linked genes and their 
modifiers, and these were ranked in a dominance heirarchy (with some codominance and a few heterozygote forms).

This genetic work is now being followed up by a molecular genetic approach by Vane-Wright et al. at the Natural History Museum, London, where they are trying to understand the evolution of the various races and morphs of Papilio dardanus (Vane Wright et al., in press). There is still a great deal of debate about how the various races and morphs that are seen in the species today came about (see Chapter 7 for a discussion of this), and it is hoped that the molecular genetic analysis will shed light on this. The work in this thesis should complement this phylogenetic work, studying the way in which the evolution may have been driven. In a wider context, the results should illustrate some of the ways in which the psychology of the signal receiver can have a profound effect on the evolution of species and on the evolution of mimicry.

\section{$\underline{\text { References }}$}

Arak, A. \& Enquist, M. 1993. Hidden preferences and the evolution of signals. Phil. Trans. R. Soc. Lond. B., 340, 207-213.

Basolo, A.L. 1990. Female preference pre-dates the evolution of the sword in swordtail fish. Science, 250, 808-810.

Basolo, A.L. 1991. Male swords and female preferences. Science 253, 1426-1427.

Basolo, A.L. 1995. A further examination of a pre-existing bias favouring a sword in the genus Xiphophorus. Anim. Behav. 50, 365-375.

Bates, H.W. 1861. Contributions to an insect fauna of the Amazon Valley. Trans. of the Linn. Soc. [Offprint]

Bates, H.W. 1864. The Naturalist on the River Amazonas. J. Murray, London.

Benson, W.W. 1972. Natural selection for Mullerian mimicry in Heliconius erato in Costa Rica. Science, 176, 936-939.

Brower, L.P., Ryerson, W.N., Coppinger, L.L., \& Glazier, S.C. 1968. Ecological chemistry and the palatability spectrum. Science, 161, 1349-51.

Carpenter, G.D.H. 1946. Mimetic Polymorphism. Nature 158, 277-9.

Clarke, C.A., Clarke, F.M.M., Gordon, I.J. Mimicry and other controversial topics in East African Lepidoptera. 1995. J. East Afr. Nat. Hist. 84, 3-19.

Clarke, C.A. \& Sheppard, P.M. 1959. The genetics of Papilio dardanus, Brown. I race cenea from South Africa. Genetics 44, 1347-1358.

Clarke, C.A. \& Sheppard, P.M. 1960a. The genetics of Papilio dardanus, Brown. II races dardanus, polytrophus, meseres and tibullus. Genetics $\mathbf{4 5}, 439-457$. 
Clarke, C.A. \& Sheppard, P.M. 1960b. The genetics of Papilio dardanus, Brown. III race antinorii from Abyssinia and race meriones from Madagascar. Genetics 45, 683-698.

Clarke, C.A. \& Sheppard, P.M. 1962. The genetics of Papilio dardanus, Brown. IV data on race ochracea, race flavicornis, and further information on races polytrophus and dardanus. Genetics 47 , 909-920.

Cook, S.E., Vernon, J.G., Bateson, M., Guilford, T. 1994. Mate choice in the polymorphic African swallowtail butterfly, Papilio dardanus: male-like females may avoid sexual harassment. Anim.

Behav., 47, 389-397.

Dawkins, M. and Guilford, T. 1995. An exaggerated preference for simple neural network models of signal evolution? Proc. R. Soc. Lond. B., 261, 357-360.

Enquist, M. \& Arak, A. 1993. Selection of exaggerated male traits by female aesthetic senses. Nature, 361, 446-448.

Fisher, R.A. 1927. On some objections to mimicry theory; statistical and genetic. Trans. R. Ent. Soc. Lond. 75, 269-78.

Ford, E.B. 1953. The genetics of polymorphism in the Lepidoptera. Advanc. Genet. 5, 43-87.

Galis, F. \& Metz, J.A.J. 1998. Why are there so many cichlid species?. TREE, 13(1), 1-2.

Gittleman, J.L. \& Harvey, P.H. 1980. Why are distasteful prey not cryptic? Nature, 286, 149-150.

Goldschmidt, R. 1945. Mimetic Polymorphism, a controversial chapter of Darwinism. Quarterly Review of Biology, 20, 147-164.

Gordon, I.J. 1987. Natural selection for rare and mimetic colour pattern combinations in wild populations of the diadem butterfly, Hypolimnas misippus L.. Biol. J. Linn. Soc., 31, 1-23.

Guilford, T. 1986. How do warning colours work: Conspicuousness may reduce recognition errors in experienced predators. Anim. Behav., 34, 286-288.

Guilford, T. 1990. The evolution of aposematism; in D.L. Evans and J.O. Schmidt (eds), Insect Defenses - Adaptive mechanisms and strategies of prey and predators, 23-63. State University of New York Press.

Johnstone, R.A. 1994. Female preference for symmetrical males as a by-product of selection for mate recognition. Nature, 372, 172-5.

Magnus, D. 1958. Experimentelle Untersuchungen zur Bionomie und Ethologie des Kaisermantels. Z. Tierpsychol. 15, 397-426.

Poulton, E.B. 1890. The colours of animals: their meaning and use especially considered in the case of insects. London, England.

Punnett, R.C. 1915. Mimicry in Butterflies. Cambridge, England.

Rothschild, M. 1981. The mimicrats must move with the times. Biol. J. Linn. Soc., 16 (1), 21-23.

Rothschild, M. 1984. Aide memoire mimicry; Ecol. Entomol., 9, 311-319.

Ryan, M.J. \& Rand, A.S. 1993. Sexual selection and signal evolution: the ghost of biases past, Phil. Trans. R. Soc. Lond. B, 340, 187-195. 
Silberglied, R.E. 1977. Communication in the Lepidoptera. In How animals communicate, ed. Sebeok, T.A. Bloomington, Indiana Univ. 362-402.

Turner, J.R.G. 1975. A tale of two butterflies. Nat. Hist., 84, 28-37.

Turner, J.R.G. 1984. Mimicry: the palatability spectrum and its consequences. in R.I. Vane-Wright and P.R. Ackery (eds), The Biology of Butterflies. Symp. Roy. Ent. Soc. Lond., 11, 141-161. Academic Press, London.

Turner, G. F. \& Burrows, M. T. 1995. A model of sympatric speciation. Proc. R. Soc. Lond. B, 260, 287-292.

Vane-Wright, R.I. 1980. On the definition of mimicry. Biol. J. Linn. Soc., 13(1), 1-6.

Vane-Wright, R.I., Raheem, D.C., Cieslak, A., Vogler, A.P. In press. Evolution of the mimetic African swallowtail butterfly Papilio dardanus: molecular data confirm relationships with $P$. phorcas and P. constantinus. Biol. J. Linn. Soc. 


\section{Chapter Two}

\section{Mate choice and colour preferences in Argynnis paphia}
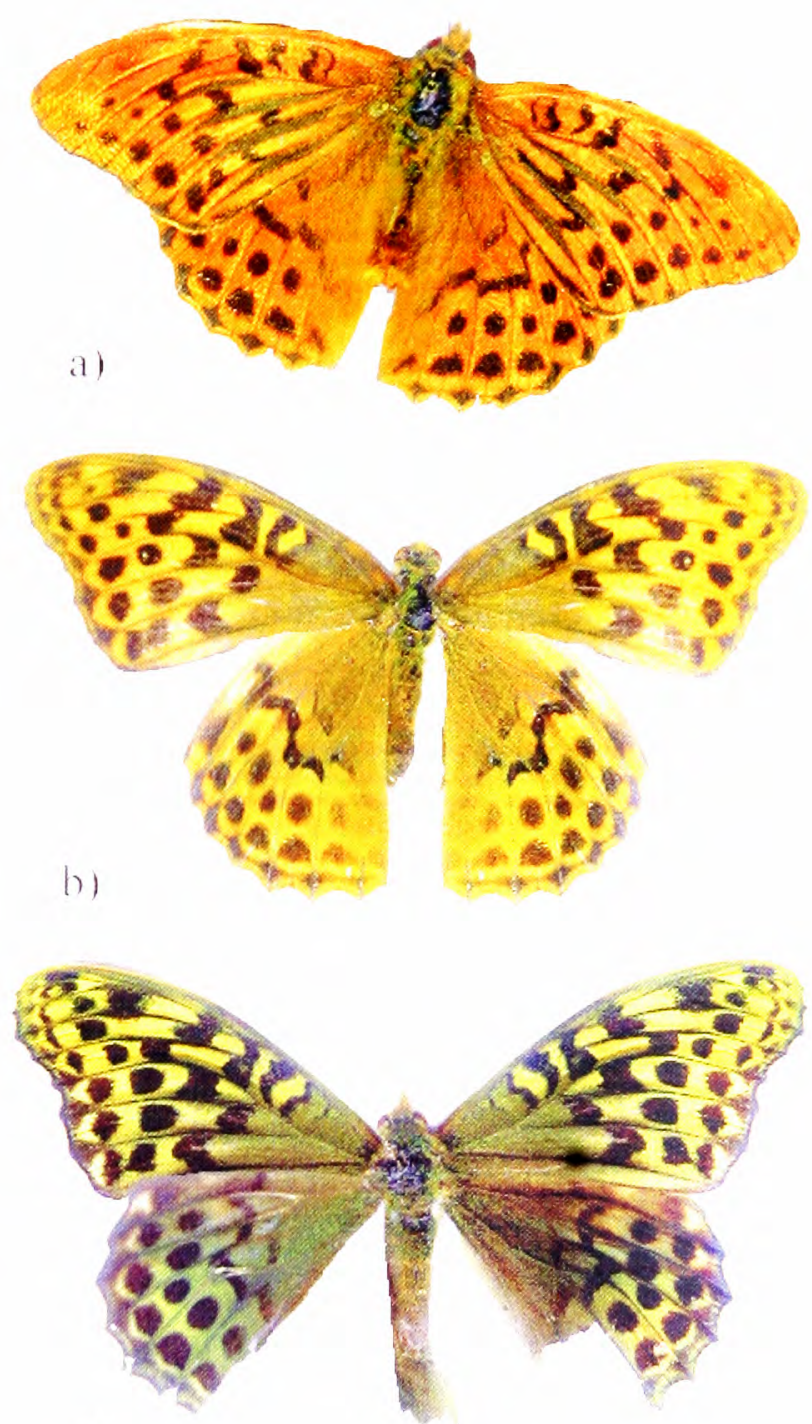

()

Figure 2-1 The Silver Washed Fritillary (Argynnis paphia): a) Male, b) Paphia female, c) Valesina female 


\section{Introduction}

This chapter investigates the basis of some interesting preferences found in a polymorphic butterfly species which appears to show a colour preference when choosing a mate and also the same colour preference when feeding from flowers. This species occurs in Britain with two population structures - one monomorphic and one polymorphic - thus presenting the opportunity to test the preferences of wild males in the two populations to see whether or not the males might be learning from their experience with the morph to which they are apparently not innately attracted. Also the presence of the same colour preference for mates and flowers is intriguing, suggesting that the males may possibly have a pre-existing bias for a colour which could have been exploited by the favoured female morph.

The Silver Washed Fritillary, Argynnis paphia, is a woodland butterfly which occurs in Britain, Europe, and across the Middle East to China (see Figure 2-1). The males and male-like females (morph paphia) are orange with black chequered markings. The sexes can be distinguished easily by the presence of black stripes on the males' forewings, which carry scent scales. The undersides of the wings are silvery green, making the butterflies well camouflaged when settled with their wings closed. There is a second female morph, valesina, which lacks the orange coloration. Instead, the upper surfaces of the wings are pale yellow or cream on the front of the forewings, changing to a dark green across the surface of the wings towards the abdomen. The frequency of valesina compared to the male-like morph, paphia, varies across the butterfly's range. In Britain valesina only occurs in central southern England, and then only at low frequency (about 5\%). This frequency increases towards the Mediterranean and Far East, until the frequency of valesina apparently reaches $100 \%$ in Southern China (Ford, 1979 p168). Genetically, the alleles are autosomal: valesina is dominant, but sperm which carry the valesina gene appear to be at some disadvantage (Goldschmidt \& Fischer, 1922).

Brunton et al. (1990) described valesina females in Corsica as approaching males, behaviour which was never seen from paphia females. This may be in order to allow the males to detect their sex pheromones if they are not visually attractive to males. It may also be normal for virgin females to show this behaviour, and that paphia females are usually mated quickly and so rarely demonstrate the approach behaviour, usually having been mated already. Brunton et al. also described how valesina females apparently had a higher heat tolerance, remaining at feeding sites during the midday heat whilst paphia females departed to the shade. This increased heat tolerance, and increased feeding times, may give the valesina females an advantage in hot countries. This increased advantage with increased temperature may explain, at least in part, the distribution of valesina, where the proportion of valesina increases approximately with increasing temperature, although the causal link is so far unexplained. 


\section{Chapter 2 Mate choice and colour preferences in Argynnis paphia}

Magnus (1958) carried out an important and extensive series of experiments on the preferences of Argynnis paphia. Since these were originally published in German and have not been discussed widely in the English-language literature, I will report his findings here.

Using inexperienced butterflies he recorded their visits to paper flowers (repeating experiments carried out by Kühn and Ilse, 1925). He described carrying out these experiments at relatively low temperatures (between 15 and $20^{\circ} \mathrm{C}$ ), and this meant that there was very little sexual activity (as measured by the frequency of the zig-zag flight characteristic of male mate-searching behaviour and approaches to other butterflies). Therefore he concluded that his results can safely be considered as flower colour preferences.

His results showed that both males and females show a preference for very bright orange/yellow (about PA3 in the Ostwald colour series), although the male peak is slightly higher and smoother, and pale blue (GA15 in the Ostwald series), where the female peak is higher. There is a lesser peak at pale green, which Magnus explained as being due to the butterflies searching for leaves on which to land in the cold.

Magnus carried out experiments on mate preference with wild butterflies. He found that there were no shape preferences and that males chose larger models over smaller models. He then investigated the attraction of moving models using an apparatus with a rotating arm in the field. He found a peak preference for orange (PA3-5), although there is also a lesser peak in the blue at PA13. Again he found that shape was not an important factor for the males and that attractiveness increased with size of the model. He then tested the effect of the model taking a flight path which fluctuated upwards and downwards (which he records as being characteristic of butterflies in 'feeding mode'), and found that models taking a straight path were preferred. Finally he tested the preferences of males for different frequencies of fluttering in the models. He found that higher frequencies of flutter were always preferred, up to the limit of the mechanism ( 40 cycles per second). He then continued the experiment with a new mechanism that spun a model around its axis of symmetry instead of trying to simulate actual fluttering. With this he recorded that males were increasingly attracted up to 50 revolutions per second. Above this they were not attracted, and he assumed this to be due to their flicker fusion frequency. Interestingly he noted that Hesperidae continued to be attracted at very high rates of revolution (sadly killing large numbers of them as they collided with the apparatus!)

Magnus then investigated the role of patterning in attracting males. He compared the normal female patterning to a model with no black at all, and one with denser, coarser, black markings and found that there was no significant difference between the attractiveness of the normal pattern and the model with no black at all, but that the model with more black was less favoured. He suggested that 
the males do not resolve the pattern at a distance, and that they rely on an 'overall effect' of colour, which was disrupted by the coarser black markings. He also tested the attractiveness of valesina against paphia females, and found that valesina was preferred only $10 \%$ of the time. However, he pointed out that the females release a pheromone which will trigger mating behaviour in the male in the absence of any colour stimulus, and so he concluded that the possession of this pheromone is what causes valesina females to be mated in the wild.

Interestingly, he then bred his own Argynnis paphia and tested the effects of genetics on the male preference for paphia females, and found that males homozygous and heterozygous for the valesina gene were indistinguishable from those homozygous for the paphia gene. This indicates that the gene, or gene complex, controlling male preference for either paphia or valesina females is not linked to the gene which causes the coloration itself. Therefore if a difference in the percentage of males with a preference for valesina is found between populations which varies in proportion to the percentage of valesina females in those populations, it cannot be ascribed to linkage disequalibrium between a gene complex controlling the coloration and one causing a preference for the coloration.

The results of the flower choice experiments, which showed that both inexperienced males and females had a preference for orange flowers (slightly greater in males than females) together with the results of the mate choice experiments, in which experienced males had a preference for orange mates (of any shape, preference increasing with size) suggests that both sexes have an innate preference for orange, and that this is being expressed in both flower choice and mate choice. However, since the males used in the mate choice experiment were wild, and therefore may well have experienced orange, paphia, females, it is also possible that they had learnt to recognise the common paphia females as mates. The $10 \%$ of males which preferred valesina in the choice experiments may correspond to those which had previously encountered valesina females (the proportion of valesina in the experimental population is not reported, although they were taken from Southern Germany where the proportion is said to be low (Brunton et al., 1990)). One way of testing the hypothesis that the preference is learnt is to repeat the mate choice experiments in two populations with varying proportions of valesina and paphia, such as in two areas of England, where it might be expected that the preference for valesina would vary with the proportion of valesina in the population.

\section{$\underline{\text { Aim }}$}

The overall aim of the experiments is to determine whether male Argynnis paphia are innately attracted to orange, paphia, females - as they appear to be to orange flowers - or whether they learn a preference between the two morphs from their previous experience of females. Once this has been determined, the aim is to understand the basis for the mechanism and its implications for the evolution and maintenance of the valesina morph. 


\section{Behavioural Observations}

All experiments were carried out in Crab Wood, near Winchester, between the $10^{\text {th }}$ July and $9^{\text {th }}$ August, 1997. The butterflies were observed for about 30 hours during this period.

The butterflies spent the nights and cloudy periods resting up in trees, and when the sun emerged, would drop down like falling leaves into the sunny clearings and rides. Both sexes fly early in the morning (before 9am BST) if sunny. Females found bramble or thistle flowers (they were also seen on cow parsley), and fed whilst males patrolled the area, flying at the height of the bushy vegetation (mostly brambles and long grass). The males flew at other butterflies on the flowers, but appeared to be repelled by a sharp flash of the wings, at which they moved on. If a female didn't react in this way, the male would get underneath her and eventually push her off the flower. She then flew in a straight line with fluttering wings, with the male circling around her from front to back. If she then accepted him, the two started a 'wheeling' flight, both continually circling each other. Both sexes flew throughout the day whilst the weather remained very sunny, although the number of females seemed to decrease between about 11 am and 3pm BST. Pairs were seen 'wheeling' throughout the day, until about 3:30pm BST, by which time there were very few Argynnis paphia around. Valesinas were seen at all times of the day, more often in the open, sunny coppiced areas. They were never seen to approach males as reported by Brunton et al. (1990), and one was seen to reject a male's advances. They were often approached by gatekeeper butterflies (Pyronia tithonus), which are about the same colour, but smaller. The proportion of valesinas was estimated to be about $7 \%$ of females through observation of females throughout the experimental period. About 150 females in total were seen during the 30 hours of observations, of which approximately 10 were valesinas. These butterflies were all thought to be different individuals, and the valesina females almost certainly were as seven of them were caught for use in the experiments and the variability in their coloration also made them individually recognisable.

Attempts were made to breed the species in captivity. The females laid eggs and small larvae emerged. In the wild these overwinter in the soil, but in captivity none re-emerged the following spring. In addition the adults proved difficult to keep alive, so all experiments were planned as fieldwork. The butterfly also appeared to be non-territorial, so repeated experiments on the same individual in the wild are not practical. 


\section{Experiment 2-1 Male mate preference: Male versus paphia female}

\section{Introduction}

If the males of Argynnis paphia are initially attracted simply by colour, they might be expected to approach pinned-out males at least as often as paphia females. The males actually appear a brighter orange than the females, so they may approach the males more often than the females. If they approach the females more often, it is likely that they are detecting the female sex pheromone over a large enough distance to affect the results (since Magnus showed that the males showed no preferences between wing patternings). If they can detect the pheromones over such a distance, this would have to be taken into account when designing choice experiments. It is also possible that the males may approach other males in an aggressive manner (especially if a female is apparently present), so the behaviour of the males to both the pinned-out males and the paphia females was observed closely.

\section{Aim}

The aim of this experiment is to determine whether or not experienced, wild, males show a difference in their behaviour to pinned-out dead paphia females and the apparently brighter males, and also to establish the distance over which the female pheromones might be acting.

\section{Method}

In order to test whether or not males discriminate between pinned-out males and pinned-out paphia females in their approach, a simultaneous presentation method was used, forcing the males to make a direct choice between a male and a female.

One dead male specimen and one dead paphia female specimen were presented in each experiment. The specimens were pinned through the thorax, with wings open, onto two corks held $50 \mathrm{~cm}$ apart with wire, elevated to the height of the surrounding vegetation to maximise the chances of them being seen by free-flying males. These were placed in feeding areas where males were seen to be flying at the time of the experiment, and the specimens were positioned so as to be obvious from all angles of approach to avoid bias in the results due to the approach direction of the males. The apparatus was observed from a distance of a few metres away so as not to disturb the butterflies, and if a wild male was seen to make a deviation in his flight-path towards one of the pinned-out specimens it was recorded as a 'choice'. When the males landed on pinned-out specimens, attempts were sometimes made to mate, with both females and males. These were interrupted, however, by the removal of the male from the pinned-out specimen, as the fluttering of a male tended to attract other individuals in the area to investigate. 
Once a male had been seen to make a choice between the two specimens, he was caught and kept in a mesh carrying-net for the rest of the day. If a male was not caught, the apparatus was moved $30-50 \mathrm{~m}$ away for the next experiment to ensure that the data points were from different males and could thus be treated as independent as males might spend some time in each feeding-patch and thus their choices be recorded more than once. The time of each encounter, the first specimen the male approached, from which direction, and whether the male landed or attempted to copulate were all recorded.

Four male specimens and four paphia female specimens (collected from the area) were used in the experiment, and each possible combination of specimens was used twice - once with the male on the left and once with the female on the left All experiments were carried out in sunny weather between the hours of $9 \mathrm{am}$ and $4 \mathrm{pm}$ BST on $10^{\text {th }}$ June, $22^{\text {nd }}$ June, $23^{\text {rd }}$ June, and $28^{\text {th }}$ June 1997.

\section{Results}

In most cases when a data point was recorded, the approaching male simply diverted his flight to fly around a pinned-out specimen briefly. In some cases, however, the male landed and showed considerable interest in the pinned-out specimen, which was noted.

\begin{tabular}{|l|c|c|}
\hline & Male & Paphia female \\
\hline number of approaches & 24 & 8 \\
\hline number of landings & 4 & 6 \\
\hline percentage of approaches resulting in landing & 16.7 & 75 \\
\hline
\end{tabular}

Table 2-1: The responses of wild Argynnis paphia males to simultaneously presented dead males and paphia females

These results indicate that significantly more males approach pinned-out males than approach pinned-out paphia females in a simultaneous choice test $(\mathrm{p}<0.0035$; binomial test; $n=32)$.

A contingency table constructed for the result of the approaches (see Table 2-2) reveals that the 'expected landings' for the paphia female are too small to allow an accurate assessment of the difference between observed and expected for this cell, making a chi-squared test liable to overpredict the difference. However, when a chi-squared test is carried out, it is revealed that the chi-squared value (4.602) is large enough to give a p-value of less than 0.05 even when the value from this cell is entirely left out of the calculation (see Table 2-2). Therefore it can be concluded safely that the wild males landed significantly more often on the paphia females than the males once they had made an approach. 


\begin{tabular}{|cc|c|c|}
\hline \multicolumn{2}{|c|}{ approach to: } & Male & Paphia female \\
\hline \multirow{2}{*}{ no landing } & observed & 20 & 2 \\
\cline { 2 - 4 } & expected & 16.5 & 5.5 \\
\hline \multirow{2}{*}{ landing } & observed & 4 & 6 \\
\cline { 2 - 4 } & expected & 7.5 & $\mathbf{2 . 5}$ \\
\hline
\end{tabular}

$$
\chi^{2}=0.742+2.227+1.633+4.9=9.503
$$

ignoring value from cell with expected value less than $5, \chi^{2}=0.742+2.227+1.633=4.602$

Table 2-2 Contingency table constructed for observed and expected approaches and landing to dead males and paphia females

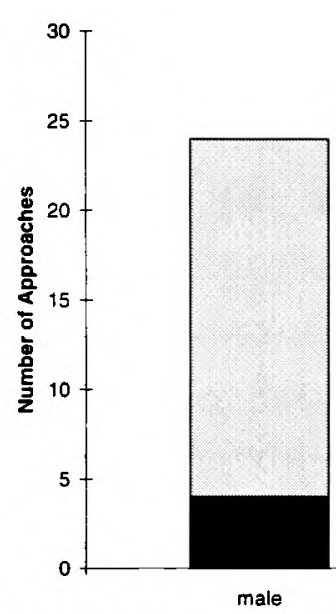

Figure 2-2 Number of approaches by wild male Argynnis paphia to both pinned-out male and paphia female specimens. The number of approaches resulting in landing is shown in black.
Many of the males approached from between the two pinned specimens, or it was not possible to determine from exactly which direction they approached. Therefore, only a small amount of data on approach direction was collected. However, as the figures in Table 2-3 suggest, the direction from which males approached appeared not to affect their choice.

\begin{tabular}{|l|l|l|l|}
\hline & $\begin{array}{l}\text { Approached from } \\
\text { right }\end{array}$ & Approached from left & $\begin{array}{l}\text { Approached } \\
\text { perpendicular to } \\
\text { experiment }\end{array}$ \\
\hline $\begin{array}{l}\text { Chose right hand } \\
\text { specimen }\end{array}$ & 3 & 5 & 4 \\
\hline $\begin{array}{l}\text { Chose left hand } \\
\text { specimen }\end{array}$ & 1 & 2 & 2 \\
\hline
\end{tabular}

Table 2-3: The direction of approach of free-flying males to pinned-out specimens

\section{Discussion}

In general, then, it can be concluded that males are more frequently attracted to pinned-out male specimens than pinned-out paphia females, but that their reaction once they had approached was different. When females were approached, the males landed (presumably to attempt copulation) significantly more often than when they approached males. Males were attracted to other patches of bright orange (such as car indicator lights), but when attempts were made to pin out painted paper models, not one male showed any response. This supports the results of Magnus (1958), that males are attracted to very bright orange, but that this attraction is lost as the orange colour pales (the painted 


\section{Chapter 2 Mate choice and colour preferences in Argynnis paphia}

models were made with water colour paint on white paper, so the orange colour had a relatively low saturation). However, at close quarters the males appear to be able to differentiate between the sexes, as they land on the females significantly more frequently. This again accords with Magnus' results, as at very close range the males can detect residual pheromones on the females, which causes the males to attempt copulation. Magnus found the colour PA4 in the Ostwald colour series to be the most attractive to the males, and showed that saturation of the colour was very important (less saturated oranges quickly lost their attractiveness). PA4 was the most saturated orange colour. Although it has not been possible to determine the spectrum of the PA4 paper, spectra could be obtained for the ground orange colour in the males and females of Argynnis paphia to confirm the apparent greater brightness (saturation) of the male colouring. This is done in Experiment 2-3.

Since Experiment 2-1 demonstrated that (outside a very close distance) males follow visual rather than pheromonal attraction clues, the experimental procedure was considered suitable for testing the relative attractiveness of paphia and valesina females to males of differing experiences.

\section{Experiment 2-2 Male mate preference: Paphia versus valesina females}

\section{Introduction}

Magnus' results (1958) together with Experiment 2-1 suggested that males might be attracted to potential mates simply through their orange coloration. However, Experiment 2-1 also indicated that at close range males can recognise mates; this is probably through the detection of pheromones, which would allow males to recognise valesina females as mates despite their coloration lacking the attractiveness of orange. Thus valesina females are usually mated in the wild, and it is possible that once a male has mated with a valesina female he might learn to recognise the valesina coloration despite the lack of an innate preference. If males are learning to recognise mates through their previous experience, then experienced males might be expected to show a preference for valesina related to the frequency of valesina in the population. The possibility that the same effect might be due to a genetic factor has already been ruled out by Magnus.

In order to test this it should be possible to carry out a choice experiment in both an area where valesina is present, and an area where valesina is absent. If a preference for valesina in some males is found in the area where they are present which is not found in the area where valesina is absent, it seems likely that learning might be occurring. The experiment could be carried out in England because valesina is present (in a low proportion) in central southern regions, and completely absent from other populations elsewhere in the country. 
The aim of this experiment is to determine whether or not males show a preference for valesina females related to the frequency of valesina in the area.

\section{Method}

The same simultaneous choice procedure was used as in Experiment 2-1, with one dead paphia female specimen and one dead valesina female specimen being presented pinned out on corks held $50 \mathrm{~cm}$ apart. The specimens were again raised to the level of the surrounding vegetation and placed in feeding areas where males were most likely to notice them. The choices of approaching males was recorded in the same way, recording the time of approach and the male's behaviour (whether he landed or attempted to copulate). As before, males were caught after each approach, and kept in carrying-nets to prevent the choice of a male being recorded more than once. If a male was not caught, the apparatus was moved $30-50 \mathrm{~m}$ away from the previous site.

Five dead specimens each of paphia and valesina females, caught in the area, were used in rotation. The females displayed varying wing conditions, but there was no difference in general between the conditions of the paphia females and that of the valesina females. Experiments 2-1 and 22 were run concurrently, with both sets of apparatus being set up and observed in the same area on some days. The trials were all carried out in Crab Wood, near Winchester, on $22^{\text {nd }} \mathrm{July}, 23^{\text {rd }} \mathrm{July}, 28^{\text {th }}$ July, and $9^{\text {th }}$ August.

\section{Results}

\begin{tabular}{|c|c|}
\hline Number of approaches to paphia female & Number of approaches to valesina female \\
\hline 25 & 8 \\
\hline
\end{tabular}

Table 2-4: The number of approaches made by wild male Argynnis paphia to simultaneously presented dead paphia and valesina females

These results indicate that males significantly prefer paphia females to valesina females ( $\mathrm{p}=0.0023$; binomial test, $\mathrm{n}=33$ ). When the results were examined closely, however, those relating to one individual valesina appeared anomalous. Seven out of the eight approaches to valesina females were to this one particular individual (number 3), and this female was preferred in 7 out of 10 of the trials in which she was used. Moreover, in all but one case, the males who chose this female landed on her, and this behaviour was similar to that seen when a female which had been freshly killed by being squeezed tightly was pinned out (which was done in the initial pilot tests). It seems likely, therefore, that this individual carried a particularly large amount of attractive pheromone (possibly she was a virgin) and that this affected the results of the experiment, whereas the other females had all been mated and carried lesser amounts of pheromone which wore off between their being killed and 


\section{Chapter 2 Mate choice and colour preferences in Argynnis paphia}

being used in the experiments. It might be argued that the five paphia females might also be carrying large amounts of pheromones, thus explaining their attractiveness to males compared with the other valesina specimens. However, firstly they were all found to be less attractive than valesina 3 in pairwise tests. Secondly, if one 'particularly attractive' valesina was found out of five, and the probability of finding an equally attractive paphia was the same, then the probability of all five paphia specimens used being 'particularly attractive' is 0.00032 . Thus this can be seen to be very unlikely.

Table 2-5 shows the data if all the results of experiments involving valesina specimen 3 are removed:

\begin{tabular}{|c|c|}
\hline Number of approaches to paphia female & Number of approaches to valesina female \\
\hline 22 & 1 \\
\hline
\end{tabular}

Table 2-5: Approaches by wild male Argynnis paphia to pinned-out paphia and valesina females with results of experiments involving valesina specimen 3 removed.

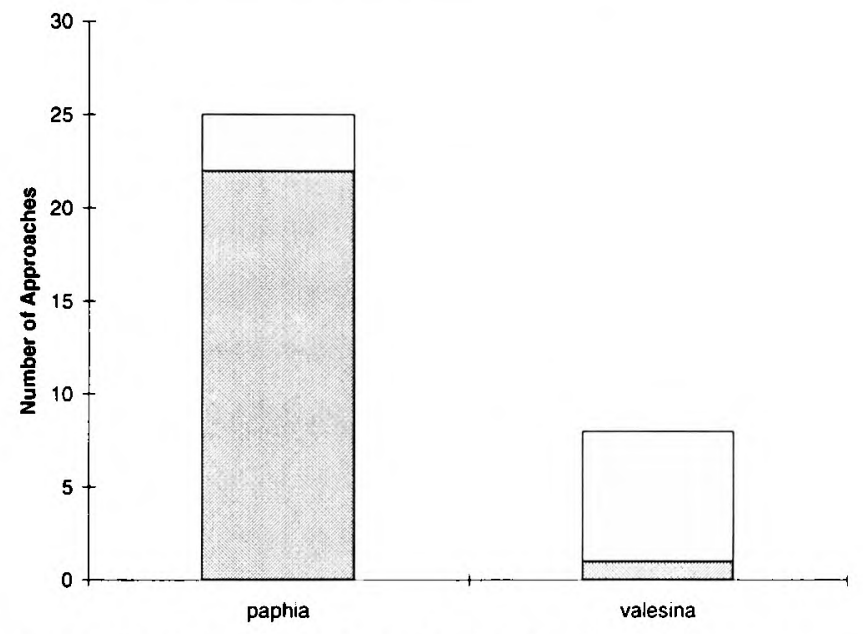

Figure 2-3 Graph showing the number of approaches by wild male Argynnis paphia to pinned-out specimens of paphia and valesina females, with the results of experiments involving valesina 3 in white.

This data gives an even stronger bias towards paphia $\left(\mathrm{p}=3 \times 10^{-6}\right.$, binomial test, $n=23$ ), showing that the males approach paphia females significantly more frequently than valesina females.

In addition, the data for the experiment involving two female morphs was obtained much more slowly than that for the experiment involving a male and a female. Since the two experiments were run concurrently in the same area for some of the time, the rate of acquisition per hour can be analysed:

\begin{tabular}{|c|c|c|c|}
\hline day & hour (BST) & male vs. female & valesina vs. paphia \\
\hline 1 & $12-1$ & 1 & 2 \\
\hline \multirow{4}{*}{2} & $10-11$ & 3 & 0 \\
\cline { 2 - 4 } & $11-12$ & 3 & 1 \\
\cline { 2 - 4 } & $12-1$ & 6 & 3 \\
\cline { 2 - 4 } & $1-2$ & 3 & 0 \\
\cline { 2 - 4 } & $2-3$ & 1 & 0 \\
\hline \multirow{5}{*}{3} & $3-4$ & 5 & 1 \\
\cline { 2 - 4 } & $9-10$ & 1 & 0 \\
\cline { 2 - 4 } & $10-11$ & 4 & 2 \\
\hline TOTAL & $11-11: 30$ & 1 & $\mathbf{1 0}$ \\
\hline
\end{tabular}

Table 2-6: The rate of acquisition of data in Experiments 2-1 and 2-2. 


\section{Chapter 2 Mate choice and colour preferences in Argynnis paphia}

The difference between the total amount of data recorded for each experiment during these time periods indicates that the male vs. paphia experiment attracted significantly more males than did the valesina vs. paphia experiment ( $\mathrm{p}=0.0025$, binomial test, $\mathrm{n}=38$ ).

\section{Discussion}

The results of Experiments 2-1 and 2-2 accord with those of Magnus (1958), showing that males seem to be attracted to the bright orange of pinned-out males and to other orange objects, although they do recognise females at close quarters as they land more often on the females than on the males, probably due to residual pheromones rather than visual recognition. This attraction for orange would explain why paphia females seem significantly more attractive to the males than the valesina females, which they rarely appear to recognise as mates at a distance. However, the data from valesina 3 might suggest that the valesina females carry the normal sex pheromone which makes them attractive to the males when the males detect the pheromone (as was postulated by Magnus). The lack of visual recognition may put the valesina at a disadvantage when they are at a distance from the males, but where males are finding females at relatively close quarters, such as at a feeding site (or if valesina females actively approach males), the pheromones are more important (as Magnus describes mating attempts occurring in the absence of any visual signals when the pheromone is present). Cook et al. (1994) suggested that female morphs which appeared not to be attractive to males at a distance may actually be at an advantage in avoiding 'sexual harassment' which could result in wing damage and lost feeding time etc. Female Argynnis paphia however were never seen to be 'harassed' unduly in the wild, and seemed to be able to feed alongside males without trouble. It does not appear, therefore, that valesina females would be at an advantage by avoiding male attentions.

Experiment 2-2 (after the removal of the anomalous data from valesina specimen 3) showed only one male out of 23 approaching a valesina female in a choice between valesina and paphia. The hypothesis that males may learn to recognise (and even prefer) valesina females as mates after an initial mating with a valesina female remains tenable, but could not be demonstrated by fieldwork in this country. Experiment 2-2, carried out in an area where valesina occurs, showed such a small preference for valesina that if the experiment were to be repeated in an area where valesina did not occur and no males approached the valesina (as would be the extreme of the hypothesis - that males only recognise valesina females as a mate after first mating with one) there would be no significant difference between the data sets. Therefore to test the hypothesis, the experiment would have to be repeated in an area where valesina occurs at a much higher frequency to determine whether or not the preference for valesina illustrated by the males increases with it. Thus it has not been possible to test for the affect of learning on the males' choice of mates. 
It is interesting that that the males appear to show identical colour preferences when searching for food and mates (the difference in their flight patterns recorded by Magnus indicating that they were showing two different behaviours). Such a preference could be due to a peak in their visual sensitivity, or it could be an innate preference which has either evolved to allow the males to recognise their mates (and/or food) or which is just the result of random drift resulting in a 'perceptual bias'. In order to clarify this area in Experiment 2-3 the colours of the male and females are compared, and in Experiment 2-4, the visual sensitivities of the butterflies are explored.

\section{Experiment 2-3 Reflectance spectra of Argvnnis paphia}

\section{Introduction}

Male Argynnis paphia show a preference for males over paphia females, and paphia females over valesina females in choice tests (Experiments 2-1 and 2-2). This preference ranking appears to be due to the differing amount of orange coloration found in the males and two morphs (Magnus 1958). The paphia females of Argynnis paphia appear to have a slightly less saturated orange background colour than the males (and the valesina females are greenish rather than orange), although it is not possible to quantify this without measuring the reflectance spectra of both male and female wings. Magnus' experiments indicated that the increased black patterning on the females' wings did not make a difference to the preferences of the males, so Experiment 2-3 is designed to quantify the orange ground colour in between the black markings of either sex.

\section{Aims}

The aim of this experiment is to quantify the apparent difference in saturation between the orange colour of the male and paphia females of Argynnis paphia.

\section{Method}

A Zeiss MC5-501 UV-Vis spectroradiometer was used to measure the reflectance spectrum of the butterfly wings. The wing was illuminated with light from a xenon bulb at $45^{\circ}$ to the normal, with measurement of the spectrum taken normal to the surface (with no integration of the scattered angular reflection). The measurements were taken of a circular area approximately $1 \mathrm{~mm}$ in diameter.

Measurements were taken of the background colour of the forewings of two specimens of either sex, and of the hindwings of two specimens of either sex. For comparison, the background colour of the forewing of one specimen of the valesina morph was also measured. The area measured was the same in each individual, and was chosen to include as little as possible of the black markings. The instrument was calibrated on an artificial true-white Spectralon ${ }^{\mathrm{TM}}$ disc, against which all the reflectivities were measured. 


\section{Results}

a) 100

90

80

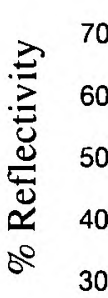

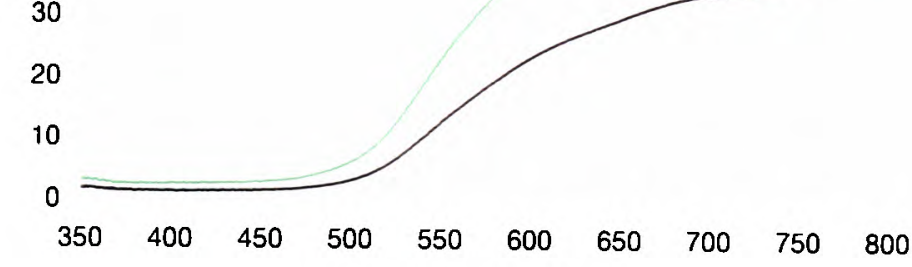

Wavelength $(\mathrm{nm})$

b) 100

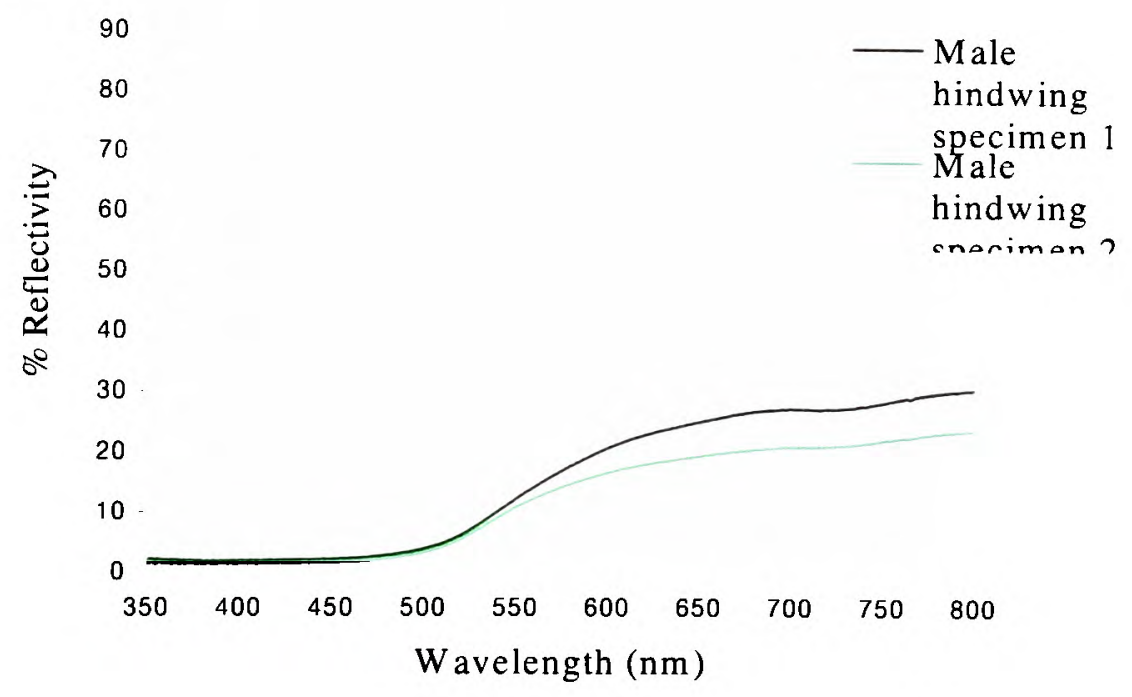

c) 100

80

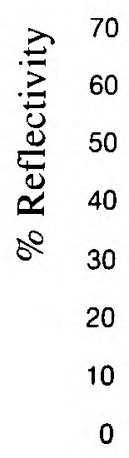

$\begin{array}{llllllllll}350 & 400 & 450 & 500 & 550 & 600 & 650 & 700 & 750 & 800\end{array}$

Wavelength $(\mathrm{nm})$ 
d) 100

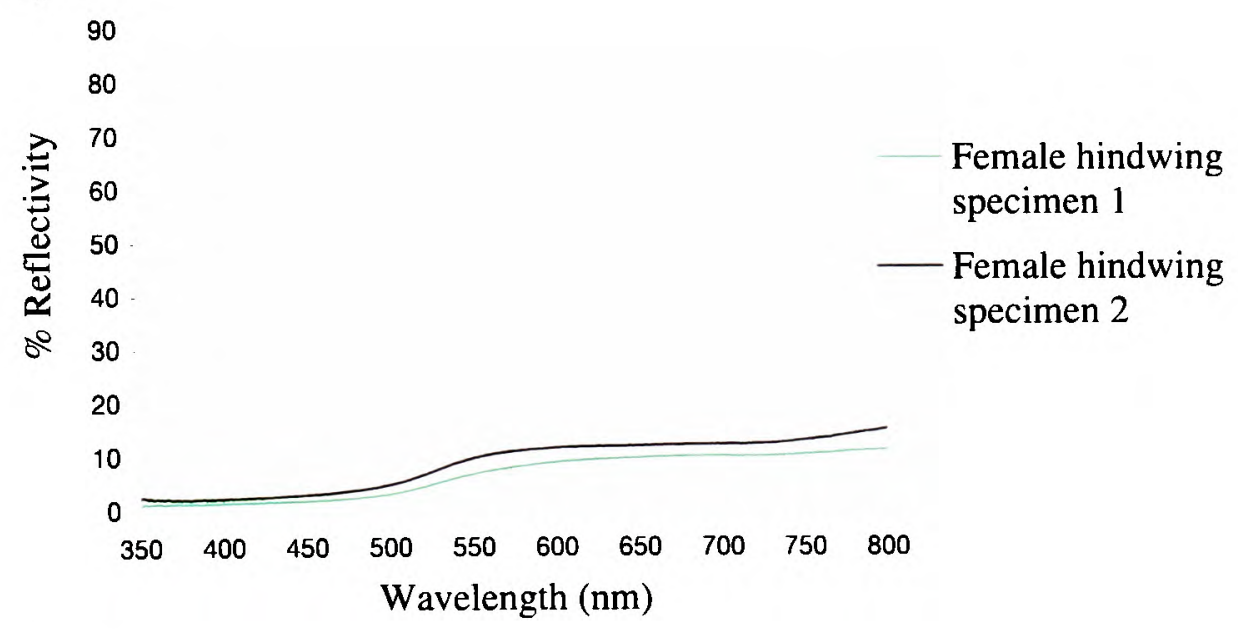

e) $100-$
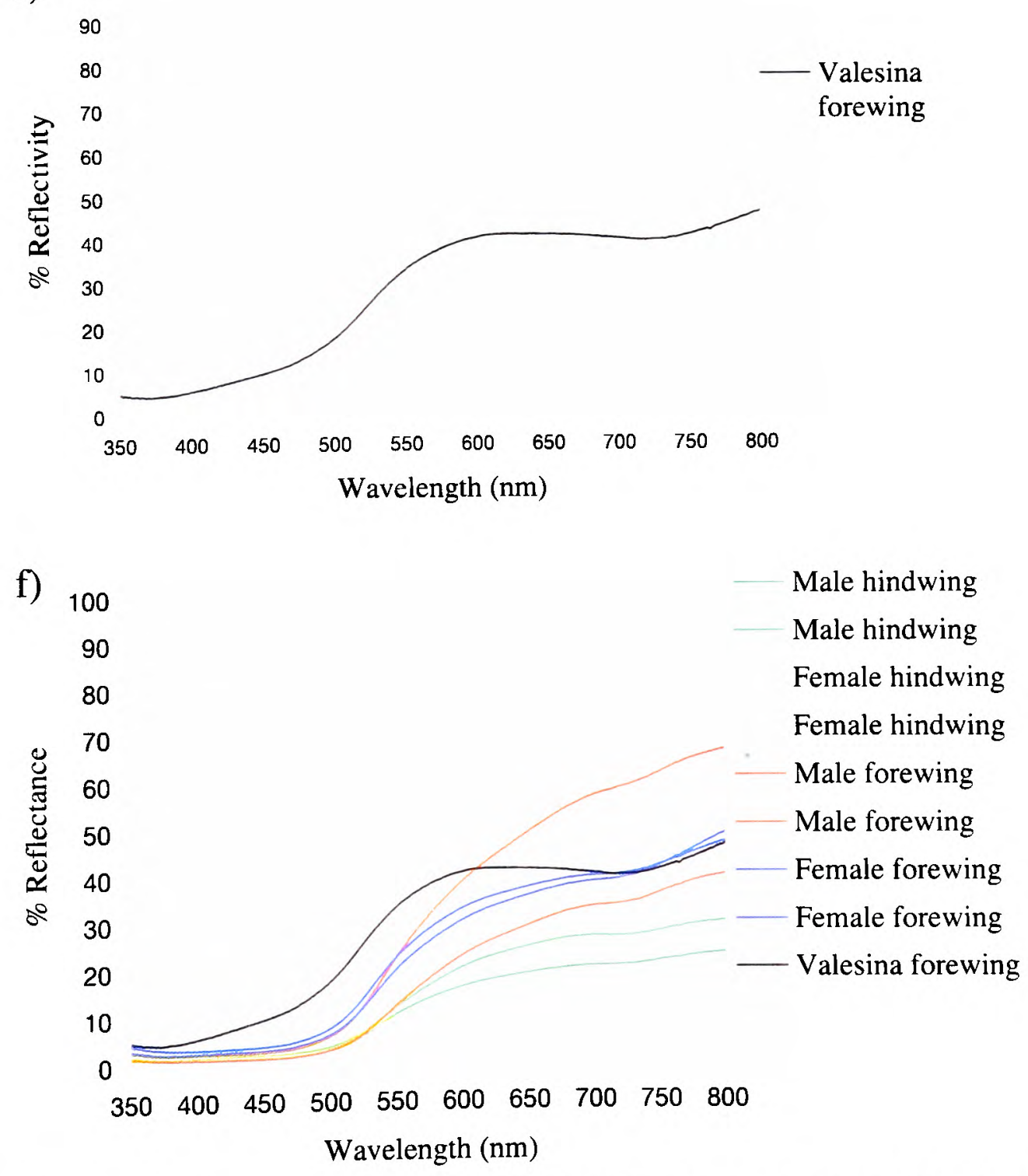

Figure 2-4 Reflectance spectra of the background colour of the wings of Argynnis paphia: a) Male forewing, b) Male hindwing, c) Paphia female forewing, d) Paphia female hindwing, e) Valesina female forewing, f) All the spectra plotted on the same chart for comparison. 


\section{Discussion}

The spectra clearly show several things. Firstly, the male and paphia female wings all show the same shape spectrum, indicating that they are essentially the same colour (hue). They all show a smooth curved decline in reflectance from $750 \mathrm{~nm}$ to around $500 \mathrm{~nm}$, resulting in an orange coloration (with no UV reflectance). The spectrum of the valesina female forewing is a slightly different shape, showing a relatively constant level of reflectance from $750 \mathrm{~nm}$ to $550 \mathrm{~nm}$, followed by a sharper decline in reflectance to $500 \mathrm{~nm}$. The reflectance between $500 \mathrm{~nm}$ and $350 \mathrm{~nm}$ in the valesina female is higher than in the males and paphia females.

Secondly, the forewings of males and paphia females show a higher reflectance than their respective hindwings, indicating a brighter colour (higher saturation). Thirdly, both the forewings and hindwings of the males are more reflectant than the forewings and hindwings of paphia females respectively, showing that the males are indeed a brighter (more saturated) orange than the females. Although one of the male forewings appears to be less saturated than both the female forewings, the two are, on average, more saturated than the two female forewings. It is possible that this uncharacteristic difference in the reflectivities of two individuals is due to a small amount of black patterning being included in the area analysed, decreasing the overall intensity.

These results therefore confirm that the males are a brighter orange than the paphia females, but shows that they are fundamentally the same colour (hue). Magnus (1958) showed that male Argynnis paphia were most attracted to the colour PA4 in the Ostwald colour system - the most saturated orange colour - and were less attracted to the less saturated oranges of the same hue (LA4, GA4). This is consistent, therefore with the results of this experiment and those of Experiment 2-1, where males were shown to be more visually attracted to other males than to paphia females.

\section{Experiment 2-4 Electroretinogram of Arqunnis paphia}

\section{Introduction}

Experiments 2-1 and 2-2, and the work of Magnus (1958) indicate that the males have a preference for bright orange coloration, and this appears to be manifested in both their mate choice and choice of flowers for feeding. This preference could be due to an increased visual sensitivity for these wavelengths of light, or a perceptual bias for this colour (which itself could either have evolved in order to allow males to detect females easily, or could be selectively neutral). It is possible to assess objectively the spectral sensitivities of animals, and thus determine whether a preference is due to the actual reception of the signal or to a bias in the processing of the signal received. 
Electroretinograms (ERGs) can be used to measure the neural response of an individual to visual stimuli, and have been used to assess the colour sensitivities of many Lepidoptera by recording the magnitude of the electrical response of the retina to flashes of light of differing wavelengths:

\begin{tabular}{|c|c|c|c|c|c|}
\hline Species & $\begin{array}{l}1^{\circ} \text { peak } \\
(\mathrm{nm})\end{array}$ & $\begin{array}{l}2^{\circ} \text { peak } \\
(\mathrm{nm})\end{array}$ & $\begin{array}{l}3^{\circ} \text { peak } \\
(\mathrm{nm})\end{array}$ & $\begin{array}{l}4^{\circ} \text { peak } \\
(\mathrm{nm})\end{array}$ & Reference \\
\hline Atrophaneura alcinous & 460 & 420 & 520 & 600 & Eguchi et al (1981) \\
\hline Papilio machaon & $<380$ & 460 & 520 & 580 & Eguchi et al (1981) \\
\hline Papilio xuthus & $\begin{array}{c}<380 \\
400 \\
\end{array}$ & $\begin{array}{l}480 \\
460 \\
\end{array}$ & $\begin{array}{l}520 \\
360 \\
\end{array}$ & $\begin{array}{l}580 \\
520 \\
\end{array}$ & $\begin{array}{l}\text { Eguchi et al (1981) } \\
\text { Arikawa et al. (1987) }\end{array}$ \\
\hline Papilio protenor & 420 & 460 & 520 & & Eguchi et al (1981) \\
\hline Papilio bianor & 460 & 520 & 420 & 580 & Eguchi et al (1981) \\
\hline Papilio maaki & $<380$ & 460 & 520 & 580 & Eguchi et al (1981) \\
\hline Papilio troilus & 483 & 578 & \multicolumn{2}{|c|}{ (UV not studied) } & Swihart (1970) \\
\hline Graphium sarpedon & 560 & 600 & $<380$ & 460 & Eguchi et al (1981) \\
\hline Colias erate & 400 & 520 & 560 & & Eguchi et al (1981) \\
\hline Gonepteryx aspasia & $<380$ & 460 & 560 & 620 & Eguchi et al (1981) \\
\hline Pieris melete & 400 & 480 & 600 & 540 & Eguchi et al (1981) \\
\hline Lycaena phlaea & 400 & 600 & 540 & & Eguchi et al (1981) \\
\hline $\begin{array}{l}\text { Lycaena rubidus \& } \\
\text { Lycaena heteronea }\end{array}$ & 360 & 437 & 500 & 568 & $\begin{array}{l}\text { Bernard \& Remington } \\
\text { (1991) - distribution } \\
\text { varies throughout eye. }\end{array}$ \\
\hline Pseudozizeeria maha & 400 & 580 & 520 & & Eguchi et al (1981) \\
\hline Celastrina argiolus & $<380$ & 440 & 560 & & Eguchi et al (1981) \\
\hline Parantica sita & 520 & $<380$ & & & Eguchi et al (1981) \\
\hline Argyronome ruslana & 560 & $<380$ & 620 & 440 & Eguchi et al (1981) \\
\hline Polygonia c-album & 560 & 600 & $<380$ & 520 & Eguchi et al (1981) \\
\hline Inachis io & 560 & $<380$ & 520 & 460 & Eguchi et al (1981) \\
\hline Aglais urticae & 360 & 460 & 530 & & Scherer \& Kolb (1987) \\
\hline Parage aegeria & 360 & 460 & 530 & & Scherer \& Kolb (1987) \\
\hline Fabriciana adippe & 580 & 520 & 460 & $<380$ & Eguchi et al (1981) \\
\hline Nymphalis xanthomelos & 560 & $<380$ & 500 & 460 & Eguchi et al (1981) \\
\hline Minois dryas & 520 & $<380$ & 460 & & Eguchi et al (1981) \\
\hline Neope goschkevitschii & $<380$ & 520 & 460 & & Eguchi et al (1981) \\
\hline Ochlodes venata & 520 & 460 & 380 & & Eguchi et al (1981) \\
\hline Parnara guttata & 520 & 460 & $<380$ & & Eguchi et al (1981) \\
\hline Cephonodes hylas hylas & 520 & 460 & $<380$ & & Eguchi et al (1981) \\
\hline Marumba sperchius sperchius & 540 & 460 & $600^{\bullet}$ & & Eguchi et al (1981) \\
\hline $\begin{array}{l}\text { Ampelophaga rubiginosa } \\
\text { rubiginosa }\end{array}$ & 540 & 460 & 580 & & Eguchi et al (1981) \\
\hline $\begin{array}{l}\text { Callambulyx tatarinovii } \\
\text { japonica }\end{array}$ & 540 & $<380$ & 460 & 580 & Eguchi et al (1981) \\
\hline Macroglossum pyrrhostictum & 500 & 460 & & & Eguchi et al (1981) \\
\hline Anadevidia peponis & 460 & 500 & 420 & & Eguchi et al (1981) \\
\hline Mamestra brassicae & 460 & 580 & 560 & $<380$ & Eguchi et al (1981) \\
\hline Samia cynthia ricini & 530 & 480 & 580 & $<380$ & Eguchi et al (1981) \\
\hline Actias artemis aliena & 540 & $<380$ & 460 & 580 & Eguchi et al (1981) \\
\hline Phassus excrescens & 440 & 400 & 520 & $<380$ & Eguchi et al (1981) \\
\hline $\begin{array}{l}\text { Arichanna gaschkevitschii } \\
\text { gaschkevitschii }\end{array}$ & 540 & 500 & $<380$ & & Eguchi et al (1981) \\
\hline Epicopeia hainesii hainesii & $<380$ & 420 & 500 & & Eguchi et al (1981) \\
\hline
\end{tabular}


These measurements were all taken with light of the same intensity (quantum flux), and the resulting graphs of electrical response of the retina against wavelength of light are known as spectral efficiency functions (Menzel, 1979 p507). However, as is pointed out by Menzel, the responseintensity function at different wavelengths can be very variable, and so an animal may seem to have a peak sensitivity to one wavelength at one intensity level may have a different peak sensitivity at another intensity level. Hence, a 'surface' of sensitivity to different wavelengths at different intensities is most meaningful, although the values at the lowest end of the intensity range are probably the most representative of the intensities reflected off surfaces (such as flowers and other butterfly wings) so the values in the table above probably give a reasonable view of the visual capabilities of the insects.

Most ERG experiments have used a 500W xenon lamp as a light source (the beam of which is then passed through a monochromator). Xenon lamps have a relatively even but fairly low intensity across the spectrum, and the quantum flux in most experiments was brought down to the level of the lowest intensity wavelength by using filters. This ensures an equal but low intensity from which to make the response measurements. Measurements of responses to higher intensities of light appear not to have been made, and a 'response surface' has not been constructed.

\section{Aim}

The aim of the electroretinogram is to determine whether the colour preferences of the butterflies can be explained by peaks in their visual sensitivities.

\section{Method}

The wings of the butterfly were removed, and the butterfly was placed in a tightly fitting plastic tube, with only the head protruding. The legs and antennae were prevented from moving by the tightness of the tube. Two thin silver wire electrodes were placed just under the surface of one of the eyes, one near the dorsal surface of the eye and one nearer the ventral surface. The impulses from the eye were amplified using an Isleworth preamplifier (amplified x 1000, no low or high pass filtering), and recorded on a Tektronix Type 502A dual-beam oscilloscope and a Lloyd Instruments PL3 chart recorder. The tube containing the butterfly was placed in an earthed metal Faraday cage to shield the electrodes from surrounding electrical appliances. The light source used was a $200 \mathrm{~W}$ mercury lamp (with an infra-red filter in the form of a lens containing continuously flowing water to prevent overheating) and a Spectral Energy GM252 monochromator, focused by a silicon lens onto the eye, and this was the only light incident on the eye within the metal box (see Figure 2-5). The light beam was interrupted by card between the IR filter and the monochromator until a steady background 
signal from the eye was obtained, also dark-adapting the eyes. The card was then sharply removed and immediately replaced, producing a spike of electrical activity from the retina.

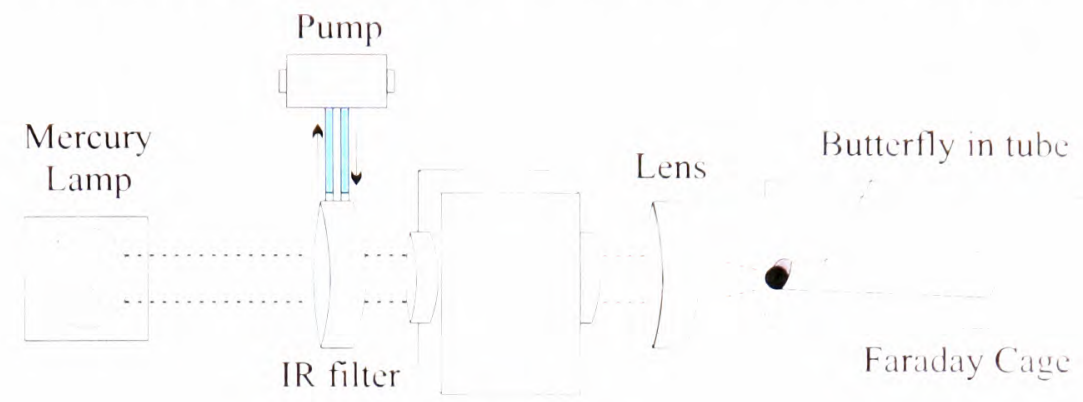

Monochromator

Figure 2-5 The experimental apparatus for measuring the ERG of a butterfly

Single measurements were taken in this way at $25 \mathrm{~nm}$ intervals through the spectrum from $250 \mathrm{~nm}$ (where no response was found) to $850 \mathrm{~nm}$ or vice versa. These 'runs' were repeated about 5 or 6 times on each individual butterfly, and the procedure was repeated for 6 individuals. Four females and two males were used (no more male specimens were available). However, no sex differences have been reported previously, and so a difference in the visual sensitivities of the sexes was not expected.

This procedure gave the responses of the retina to light of the highest intensity which could be produced by the mercury lamp (much higher than a corresponding xenon lamp). However, the intensity of a mercury lamp is by no means constant across the spectrum, so this was measured using a photodiode, RS stock number 305-462 (RS Electronics) (see Appendix 2 for further details). A set of filters (numerical swatch set, Lee Filters ${ }^{* *}$ ) was then calibrated using the photodiode at the peak wavelengths of the lamp $(375 \mathrm{~nm}, 450 \mathrm{~nm}, 550 \mathrm{~nm})$, which also correspond roughly to the peak sensitivities of the three photopigments found in Hymenoptera (Chittka \& Menzel, 1992; Backhaus \& Menzel, 1992), Manduca sexta (Bennett et al., 1997), three of the four pigments found in Lycaena butterflies (Bernard \& Remington, 1991), and three of the five pigments found in Papilio xuthus (Arikawa et al., 1987). The response of four individual butterflies was thus recorded in the same way to differing intensities of these three wavelengths. Since these showed that there was little or no variation in the intensity functions of different individuals, and that the data formed smooth curves with little noise, the surface was constructed using multiple readings from only one individual. The advantage of this was that the butterfly was not moved throughout the series of readings, and so the same proportion of the beam was falling on the eye at all times from the lamp. If different individuals had been used and placed in the beam, then inevitably some would have received slightly more or slightly less of the beam's intensity, and therefore the readings from different individuals would all be slightly shifted up or down the intensity curve relative to each other (see Appendix 2 for full details). 


\section{Results}

The wave-form of a typical lepidopteran ERG is described as a monophasic negative response (Eguchi et al., 1982). This is seen as a characteristic 'spike' above background noise in response to light stimulation (see Figure 2-6). The spikes of activity from the eye are quite difficult to analyse as occasionally an initial positive response is seen which is a response from the optical ganglion layers (Swihart \& Gordon, 1971; Eguchi et al., 1982). Where this occurred, the positive response was ignored.

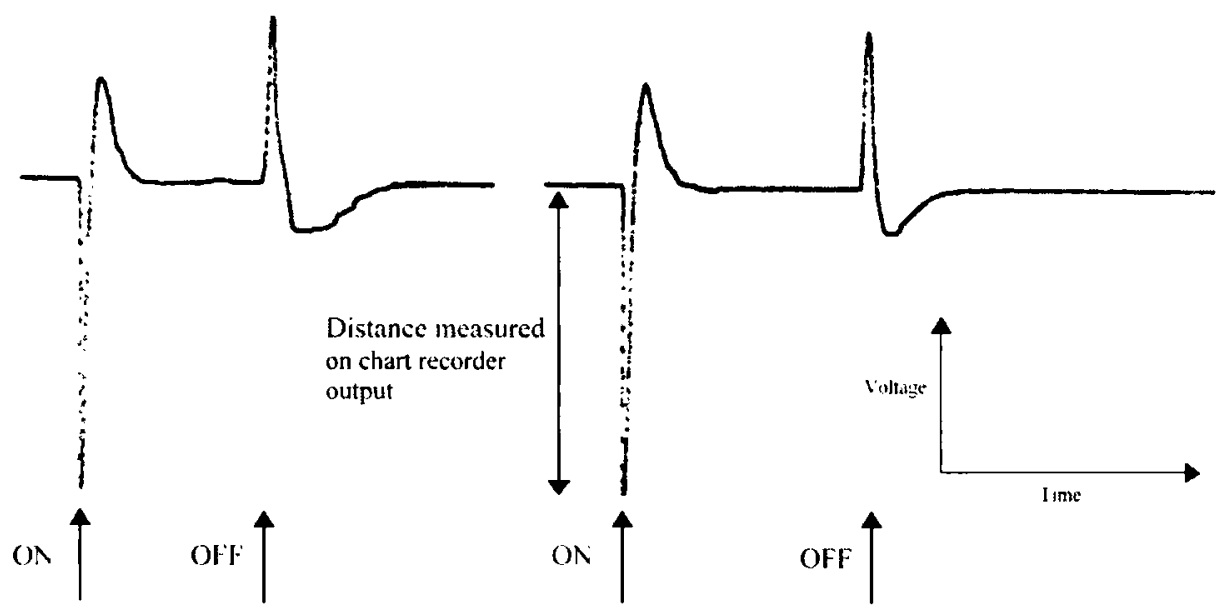

\section{Figure 2-6 Examples of the spikes produced by exposure of the eye to a flash of light}

All the individuals showed a very similar spectrum of sensitivity to wavelength, but on varying scales of amplitude. The individual spectra were therefore normalised with respect to each other. This was done by selecting one spectrum (the one in which the component 'runs' were most similar), and calculating the ratio of each point in an individual spectrum to the respective point in the selected spectrum. The mean ratio between each spectrum and the selected spectrum could then be calculated. Each point in each individual spectrum was then multiplied by this mean ratio for the particular spectrum to scale all the spectra with respect to that selected. Then a mean spectrum for all individuals was calculated. To avoid biasing the result due to the initial selection of one spectrum to which to normalise all the others, this mean spectrum was then used as the 'selected spectrum' and the process iterated until a converged mean spectrum was achieved (see Figure 2-7). 


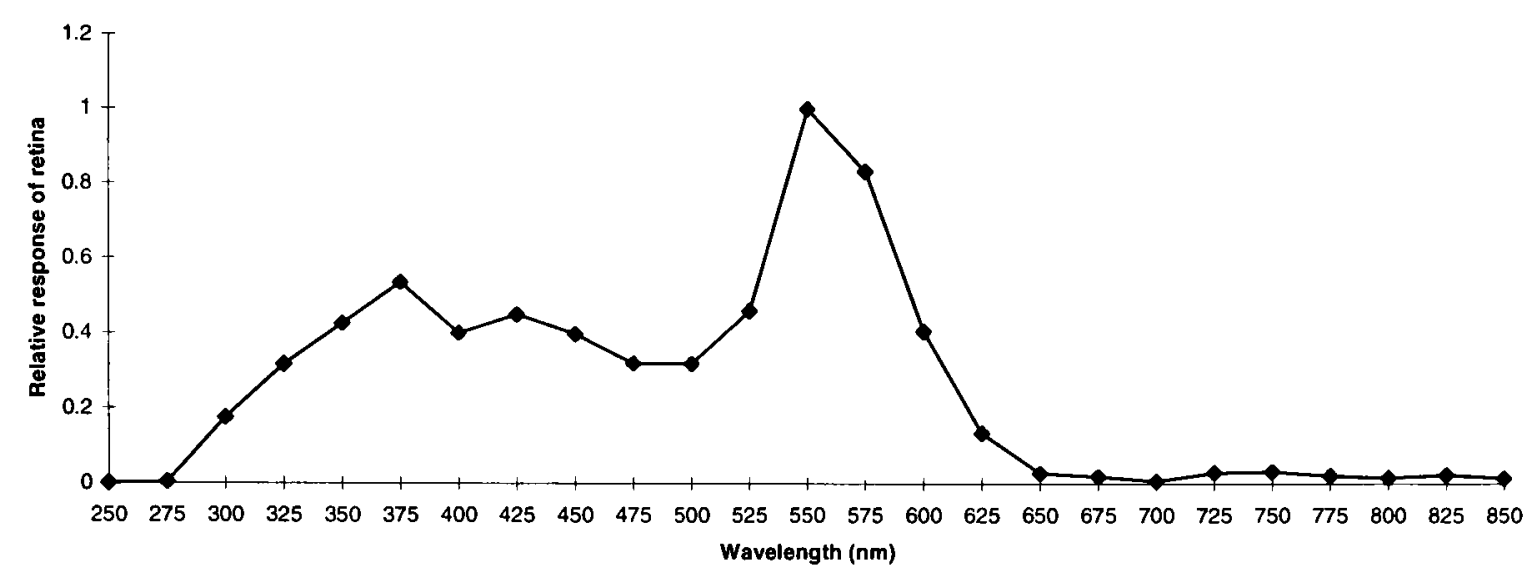

Figure 2-7 The mean response curve for Argynnis paphia belore calibration for equal intensities at each wavelength

This spectrum was then calibrated for lamp intensity. For this, measurements from another species, Papilio dardanus, had to be used due to the unavailability of further Argynnis paphia.

Although this is not ideal it is justifiable, as the similarity between the two uncalibrated response curves (see Experiment 3.5 for efficiency spectrum of Papilio dardanus) suggests that the two species are similar in their responses to different wavelengths and intensities. See Appendix 2 for further details on the calibration of the spectrum.

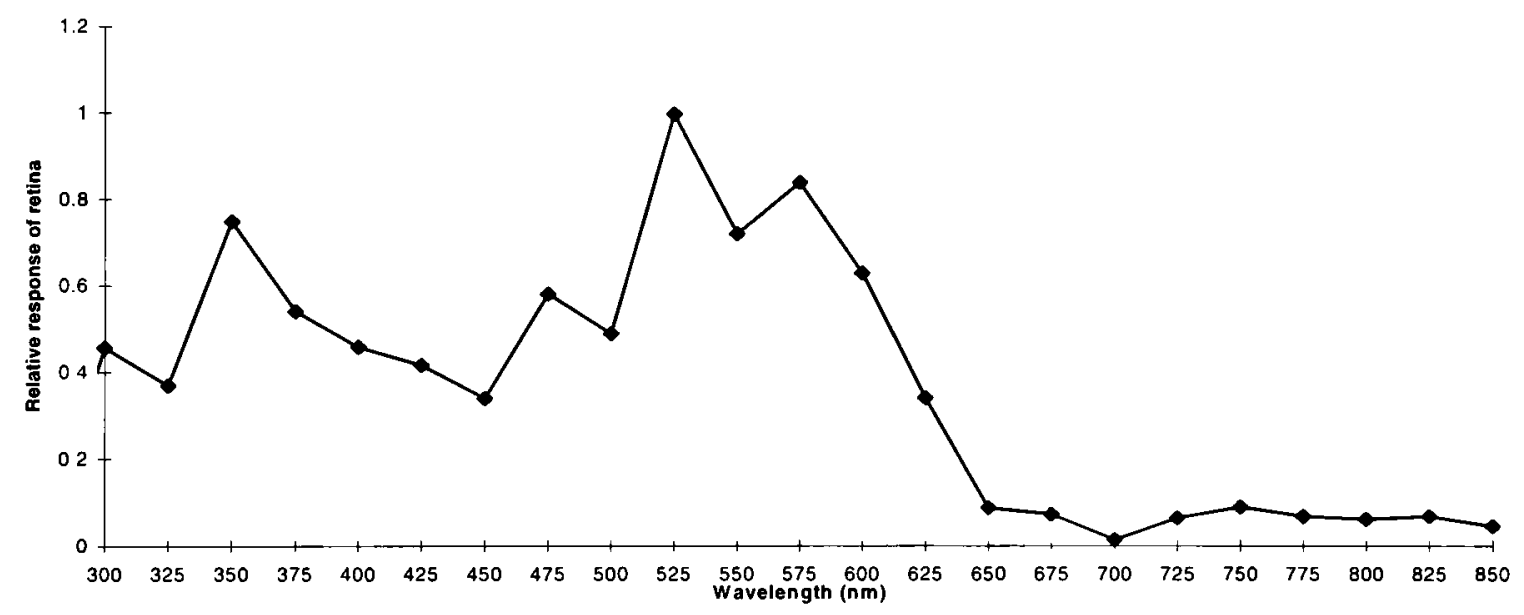

Figure 2-8 The spectral efficiency function of Argynnis paphia

\section{Discussion}

Although the spectral efficiency function may be slightly biased by the fact that the intensity spectra used were measured from Papilio dardanus, it is probably a fair estimate of the visual capabilities of the species. The three main visual pigments found in insects are present, at approximately $360 \mathrm{~nm}$ (350nm peak), $460 \mathrm{~nm}(475 \mathrm{~nm}$ peak), and $520 \mathrm{~nm}$ ( $525 \mathrm{~nm}$ peak) (Chittka \& Menzel, 1992; Backhaus \& Menzel, 1992; Bennett et al., 1997; Bernard \& Remington, 1991), with another, slightly broader peak at 575nm similar to that found in Papilio dardanus (Experiment 5-3), Papilio xuthus (Arikawa et al., 1987), Papilio aegeus (Matic, 1983), Papilio troilus (Swihart, 1970) and others in Table 2-7). 
The presence of response to wavelengths above $700 \mathrm{~nm}$ is intriguing. The response is very small compared with that found between 300 and $650 \mathrm{~nm}$, but all the same it was quite marked in many individuals, whilst extinction of the response was achieved at $250 \mathrm{~nm}$ and below (see Figure 2-7 - the response below $300 \mathrm{~nm}$ is not shown in Figure $2-8$ as the sensitivity of the light meter was too low at these wavelengths to produce a meaningful calibration). Above $700 \mathrm{~nm}$, the structure of the eye should filter out most if not all of the incident light, and thus render detection by the animal nearly impossible. In addition, photopigments that respond to such wavelengths are very rare (although Douglas et al. 1998 have identified some chlorophyll derivatives such as pheophorbide a and pyropheophorbide a which have absorption spectra around 700nm and above, and have shown their use in the dragon fish Malacosteus niger). It seems unlikely that the butterflies would indeed be sensitive to this region of the spectrum, and this result could simply be due to a fault in the particular monochromator used which let light of wavelengths other than 750-850nm through, but no others were available to repeat the experiment. It should also be noted that the when the monochromator was set to wavelengths between $750-850 \mathrm{~nm}$ no light of other wavelengths visible to the human eye were seen to be passing through.

Comparing this spectrum of sensitivity with the reflectance spectra of the wing colours of the species it can be seen that the preference for the orange of males and paphia females over the greener coloration of the valesina females cannot be ascribed to a visual bias for the colour orange. In fact, the valesina females might be expected to be more attractive as they have a higher reflectance around $525 \mathrm{~nm}$, the peak sensitivity for the species. Therefore the preferences shown by the males must be due to higher neural processing.

\section{Conclusions}

Male Argynnis paphia appear to be attracted to highly saturated orange coloration, both when choosing mates and flowers (Magnus, 1958). Their preference for the most highly saturated orange accords with them being more attracted to the brighter orange males than the less highly saturated orange paphia females (results of Experiment 2-1), and not visually attracted to the greener valesina females (results of Experiment 2-2). The apparent difference in saturation between the males and the paphia females has been confirmed by reflectance spectroscopy (Experiment 2-3).

This preference appears not to be due simply to a visual sensitivity bias in the retina of the butterfly (results of Experiment 2-4), and so seems to be due to the perception rather than the reception of the signal. This preference must either have been selected for in evolution or be due to random, unselected drift.

If the preference were initially due to random drift, it could have then been exploited by the paphia females in order to increase their visual attractiveness to males. The fact that they are not as 
bright (and visually attractive to males) as the males could be due to a trade-off between this and the need to be cryptic, in order to lay as many eggs as possible, although it is unclear why in this case the males should have evolved an orange coloration (and one which is slightly different from that of the females). The fact that Magnus (1958) showed a lesser reaction to the bright orange in the females indicates that the roles are unlikely to be reversed - with the males becoming brighter in order to attract females. The sex difference in perception also suggests that the preference may have been selected for in the males. If this were the case, the bias would have evolved as those individuals who reacted to orange stimuli particularly strongly achieved more successful matings with the orange females and left more offspring than those individuals who did not.

It is not easy to differentiate between the adaptive and random drift possibilities, nor to determine whether or not the females are showing sensory exploitation of the males. It would be very interesting to repeat the colour preference experiment with males from an area where valesina is the most common form of the female (such as in Southern China) to determine whether or not the males retain their preference for orange paphia females, or whether they have an innate preference for the valesina coloration. If they still retain the, now maladaptive, preference for orange mates then it would seem more likely that the preference is due to random drift. It would also be interesting to look at the phylogeny of the colour preference, to determine whether it is shown in other related fritillaries (especially those where the ground colour is not the same bright orange).

Since Magnus' results show that the males show some interest in blue models, it might be possible for a blue female morph to exploit this attraction. In this species it seems unlikely that this would occur as the females must remain cryptic. However, it is be possible that such a bias might be exploited in a polymorphic mimetic butterfly such as Papilio dardanus. Therefore the flower colour preferences of Papilio dardanus are investigated in Chapter 4, in order to compare these with the mate colour preferences found by Cook et al. (1994), and also further investigated in Chapter 5.

\section{$\underline{\text { Acknowledgements }}$}

I would like to thank Hampshire County Council for allowing me to carry out the field work in Crab Wood. 1 would also like to thank Innes Cuthill for the use of the spectroradiometer, and Richard Compton for the use of the xenon lamp, monochromator and chart recorder. I am deeply indebted to Frank Marken for help with translations from German, and to Steve Simpson and John Alden for help with the ERG.

\section{References}

Arikawa, K., Inokuma, K., Eguchi, E. 1987. Pentachromatic visual system in a butterfly.

Naturwissenschaften 74, 297-298. 
Backhaus, W. \& Menzel, R. 1992. Conclusions from the colour vision of insects. Behavioral and Brain Sciences 15 (1), 28-30.

Bennett, R.R., White, R.H., Meadows, J. 1997. Regional specialisation in the eye of the sphingid moth Manduca sexta: blue sensitivity of the ventral retina. Visual Neuroscience 14, 523-526.

Bernard, G.D. \& Remington, C.L. 1991. Color vision in Lycaena butterflies: spectral tuning of receptor arrays in relation to behavioural ecology. Proc. Natl. Acad. Sci. USA 88, 2783-2787.

Brunton, C.F.A., Baxter, J.D., Quartson, J.A.S., \& Panchen, A.L. 1990. The valesina morph of the butterfly Argynnis paphia (L.) in Corsica, 1988. Ent. Rec. 102 (1-2), 31-7.

Chittka, L. \& Menzel, R. 1992. The evolutionary adaptation of flower colours and the insect pollinators' colour vision. J. Comp. Physiol. A 171, 171-181.

Cook, S.E., Vernon, J.G., Bateson, M. \& Guilford, T. 1994. Mate choice in the polymorphic African swallowtail butterfly, Papilio dardanus: male-like females may avoid sexual harassment. Anim. Behav. 47, 389-397.

Douglas, R.H., Partridge, J.C., Dulai, K., Hunt, D., Mullineaux, C.W., Tauber, A.Y., and Hynninen, P.H. 1998. Dragon fish see using chlorophyll. Nature 393, 423-4.

Eguchi, E., Watanabe, K., Hariyama, T., Yamamoto, K. 1981. A comparison of electrophysiologically determined spectral responses in 35 species of Lepidoptera. J. Insect Physiol. $28(8), 675-682$.

Ford, E.B. 1979. Ecological Genetics. Chapman \& Hall, 166-8.

Goldschmidt, R. \& Fischer, E. 1922. Argynnis paphia-valesina, ein fall geschlechtskotrollierter Verebung bei Schmetterlingen. Genetica 4, 247-278.

Kühn, A. \& Ilse, D. 1925. Die Anlockung von Tagfaltern durch Pigmentfarben. Biol. Ztrbl. 45, 144149.

Magnus, D.B.E. 1958. Experimentelle Untersuchungen zur Bionomie und Ethologie des Kaisermantels. Z. Tierpsychol. 15, 397-426.

Mati , T. 1983. Electrical inhibition in the retina of the butterfly Papilio. Four types of photoreceptors. J. Comp. Physiol. 152, 169-182.

Menzel, R. 1979. Spectral sensitivity and color vision in invertebrates in Comparative physiology and evolution of vision in invertebrates ed. Autrum, H. \& Myrick, E. (Handbook of sensory physiology v. VII/6A), Springer-Verlag, Berlin, 503-580.

Menzel, R. 1985. Colour pathways and colour vision in the honeybee in Central and peripheral mechanisms of colour vision ed. David Ottoson \& Semir Zeki (Wenner-Gren Center international symposium series; v.43), 211-233.

Scherer, C. \& Kolb, G. 1987. The influence of color stimuli on visually controlled behavior in Aglais urticae (L) and Parage aegeria (L) (Lepidoptera). J. Comp. Physiol. A 161 (6), 891-898.

Swihart, S.L. 1970. The neural basis of colour vision in the butterfly Papilio troilus. J. Insect Physiol. 16, 1623-1636.

Swihart, S.L. \& Gordon, W.C. 1971. Red photoreceptor in butterflies. Nature 231, 126-127. 


\section{Chapter Three}

\section{Analysis of the visual signals in Papilio dardanus}
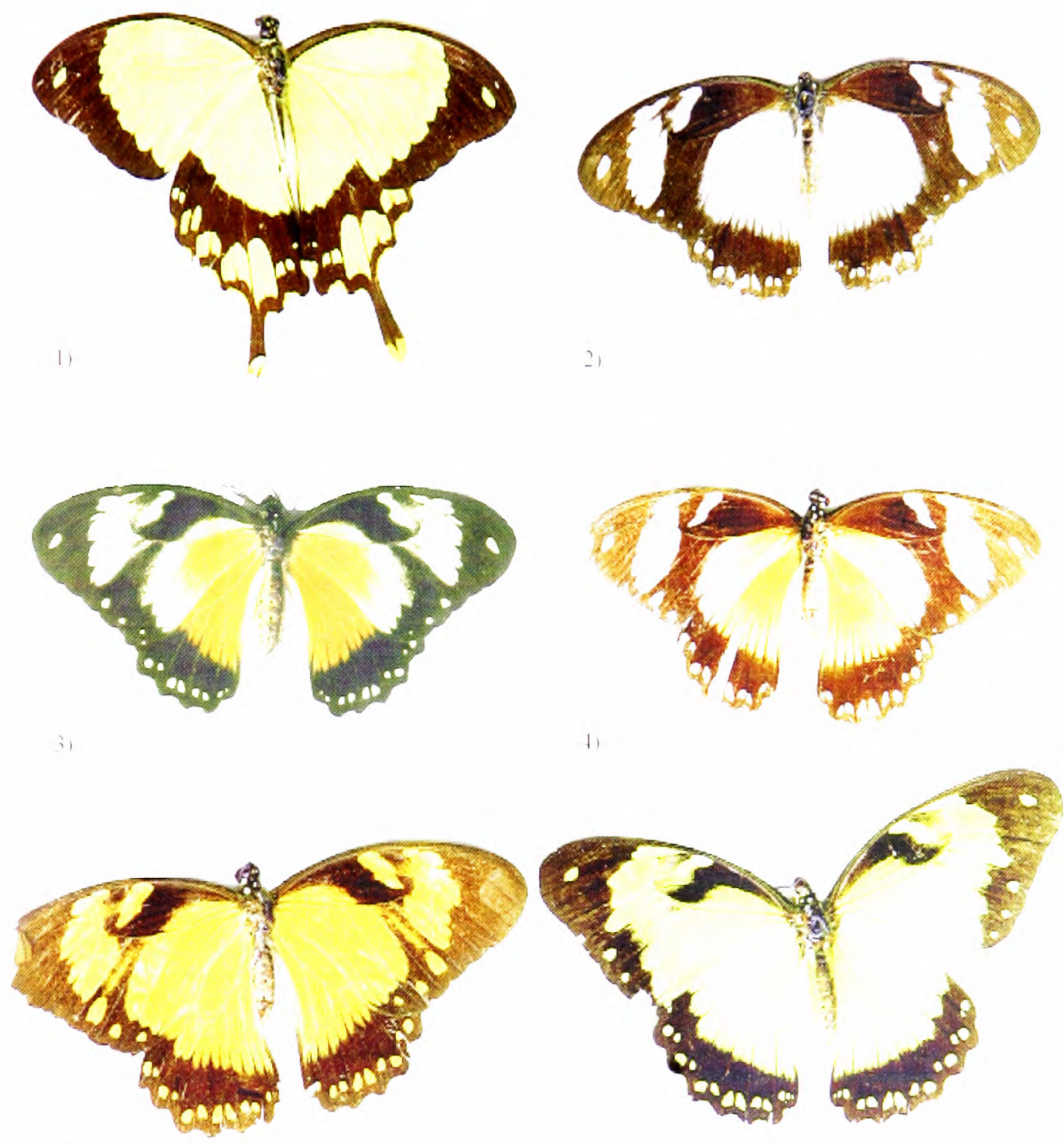

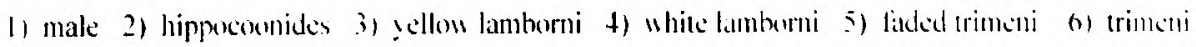

Figure 3-1 The different morphs of Papilio dardanus (race tibullus from Pemba, collected by Cook et al., 1994)

"It may be said, therefore, that on these expanded membranes Nature writes, as on a tablet, the story of the modification of species, so truly do all changes of organisation register themselves thereon. Moreover, the same colour-patterns of the wings generally show, with great regularity, the degrees of blood-relationship of the species." 


\section{Introduction}

When studying visual signalling and perception in a species it is important to understand what visual signals they may be receiving from each other and from their surroundings in general.

Butterflies have the greatest range of spectral sensitivity known for any animal. Some range from approximately 200nm (Ultra Violet) to 700nm (near Infra Red) (Bernard, 1979), as they often possess four visual pigments - sensitive in the red, green, blue, and UV regions. The 200-400nm UV range has been shown to be particularly important for butterflies, and UV markings overlay patterns in the Visible spectrum in many species (e.g. Silberglied, 1979; Brunton and Majerus, 1995). This is due to the two ways in which colours can be formed in butterflies - pigmental and structural.

Butterfly wings are made up of a lower and upper membrane, both of which are covered in scales overlapping like roof tiles. Each scale can be coloured, and the overall colour of the wing is determined by the mosaic of coloured scales. These scales can contain different pigment chemicals which cause the colours that we can see in the Visible spectrum, and also occasionally in the UV range. UV light can also induce fluorescence in the Visible range, although this is usually comparatively very much weaker than colours produced by reflectance (Cockayne, 1924). In addition to the pigment which it contains, each scale can also have a 'structural colour' caused by the way its surface scatters incident light. Scale structure was first thoroughly studied by Mason (1926, 1927a, 1927b), and more recently has been studied in great detail by Ghiradella (Ghiradella et al., 1972; Ghiradella, 1974; Ghiradella \& Radigan, 1976; Ghiradella, 1984, 1985, 1989, 1991, 1994). The scales are remarkably complex in design (see Figure 3-2), consisting of a smooth basement lamina and an upper lamina which is thrown into a series of ridges. The ridges are made up of stacks of lamellae, and these stacks are fringed by microribs.

Structural colours can be formed in a variety of ways. Ghiradella $(1984,1985$, summarized in 1991) details six methods which have been identified. Most butterfly groups use only one or two of these methods, but the Papilionidae are unique in displaying all six (Ghiradella 1985). The most common method of producing a structural colour is for either the lamellae forming the ridges, or the microribs joining them, to be exaggerated, forming a stack of layers of chitin alternating with air. The exaggerated lamellae in the first of these methods give the ridges a characteristic 'Christmas tree' structure in cross-section (see Figure 3-2a). Light incident on these structures is reflected off each one in turn, giving rise to multiple thin-film interference (see introduction to Experiment 3-4 and also Land, 1972 for a description of this). The overall wavelength reflected depends on the number and thickness of the layers and the air-spaces between them. Examples of this form of iridescence are seen most spectacularly in the South American blue Morpho species (Mason, 1927a; Bingham et al., 1995); but with very fine lamellar spacing, UV reflectance can be achieved, as seen in Colias and Eurema 
species (Ghiradella et al., 1972). In some cases a pigmental coloration and an overlying structural coloration can be combined to form a different hue, such as metallic violets - blue iridescence combined with a red pigment (Nijhout, 1985).

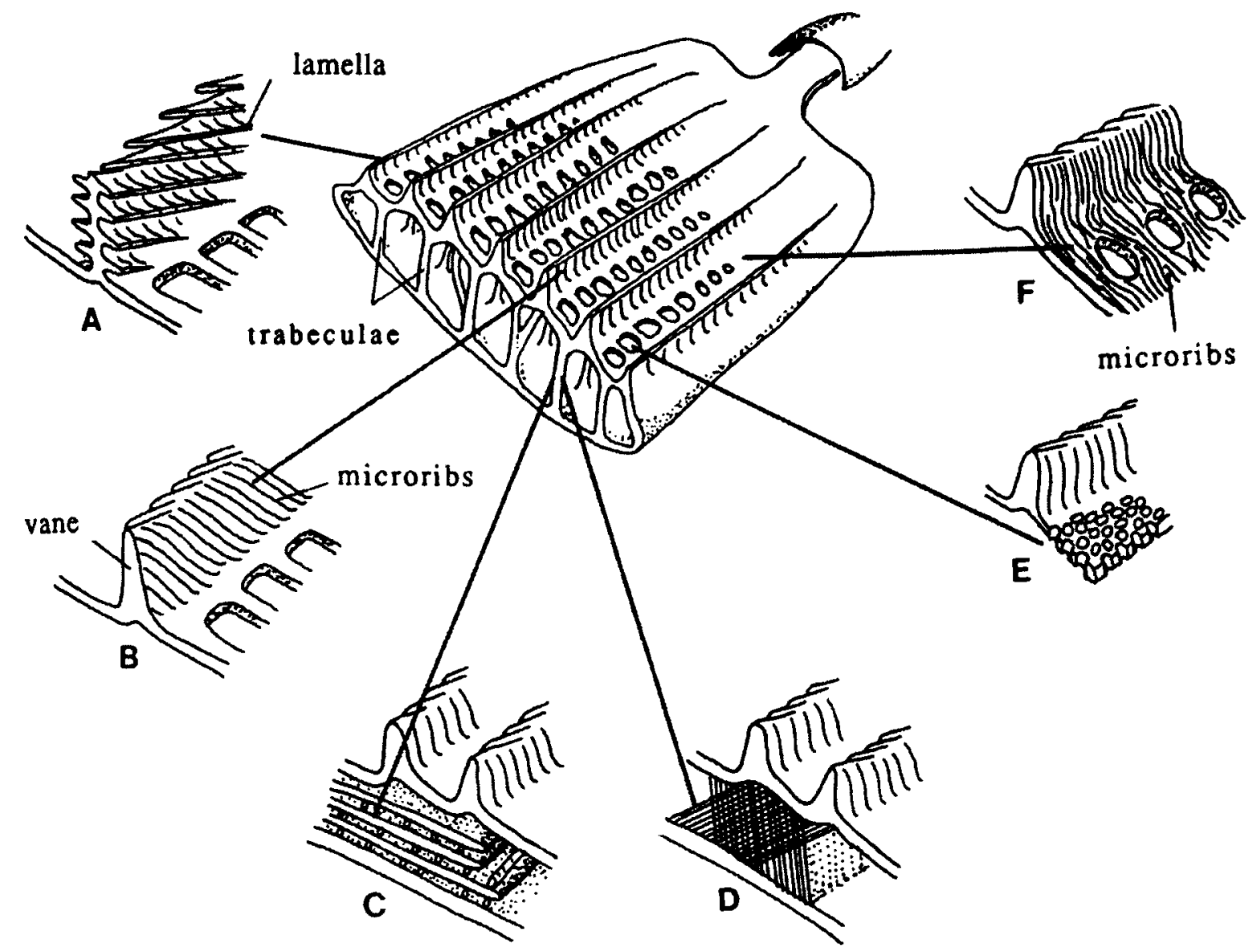

Figure 3-2 The structure of a scale and different methods of producing structural colours (from Nijhout, 1991, p13; after Ghiradella 1984, 1985). A - Lamellar thin-film interference, B - microrib thin-film interference, C - laminar thin-film interference, D diffraction lattice interference, $\mathrm{E}$ - Tyndall blue, $\mathrm{F}$ - microrib satin iridescence.

The yellow pigment seen in males (and some females) of Papilio dardanus, Papiliochrome II (Umebachi \& Yoshida, 1970), is known to fluoresce in the Visible region of the spectrum when illuminated with UV light (Cockayne, 1924). Other than this pigment, the colours found in this species appear not to have been studied in detail. Cook et al. (1994) reported that older males in the field became increasingly orange rather than yellow, and this accords with Cockayne's description $(1924$, p6) "This yellow readily changes to a browner tint and becomes non-fluorescent", but this potentially important age cue appears not to have been studied further at all. In Chapters $4 \& 5$, the colour perception of the butterflies is investigated with respect to their choice of flowers and mates, and so in this chapter their signal reception is studied - what signals are they actually likely to receive from each other?

In addition, in order to speculate which butterflies are mimics of which it is especially important to have an idea of how the butterflies may appear to their main predators. In this case the main predators are assumed to be birds, which also have a colour reception system which allows them to perceive UV coloration (e.g. see Chen \& Goldsmith, 1986). Thus, for an assessment of the visual 
signals made by the butterflies to conspecifics and to predators, a quantitative measure of their patterns and coloration is required throughout their visual range (approximately $200-700 \mathrm{~nm}$ ). It is possible to do this in an accurate and quantitative manner using a spectrophotometer, but the method is complicated by the directional nature of the structural colours which occur in Papilio dardanus. These colours may be lost or inaccurately assessed if the spectrum is measured at only one angle to the direction of illumination (as was done in the analysis of the colours in Argynnis paphia in Experiment 2-3 where pigmental colours only were being studied). This can be overcome in one of two ways. The first is to measure the reflectance off the wings at a wide range of angles. This is practically quite difficult, and very time-consuming. It also produces a very large amount of data which is difficult to interpret. The second method is to use an integrating sphere, which captures and sums the reflection at every angle. Although the latter is a more robust method, it fails to resolve the directional component of the structural colours. If the structural colours are caused by the mechanism of thin-film stack interference, in which the light hits a stack of lamellae and is scattered by reflecting off each surface in turn (see Experiment 3-5 for a more detailed discussion of this) it should be possible to predict from measurements of the thicknesses and spacing of the lamellae how the structural colours would appear.

In order to understand butterflies' perception of colours it is necessary to gather quantitative data not only on the colours of the various areas of their wings, but also on their colour reception capabilities. The electrical response of retinal neurones to light of different wavelengths can be measured quantitatively (Autrum, 1958), and this allows an objective assessment of what the butterflies actually see. Only when this is known can real conclusions be drawn about any colour preferences they may demonstrate in behavioural experiments and therefore about their perception of colours.

\section{$\underline{\text { Aims }}$}

The main aims of this investigation are:

- To measure the spectra of the different colours present in various morphs of Papilio dardanus.

- To investigate how the structural colours are produced, and from this to attempt to predict them using a mathematical model of the mechanism.

- To measure the sensitivity of Papilio dardanus to a range of wavelengths of light. 
From these results it is hoped to be able to:

- Assess how the butterflies might see different colours (important for the flower choice experiments in Chapter 4).

- Assess how the males might see the different female morphs (important for the mate choice experiments in Chapter 5).

- Understand more fully the change in coloration of the yellow pigmented areas with exposure to sunlight reported by Cook et al. (1994), and how this might be seen by conspecifics (again important for the mate choice experiments in Chapter 5).

- Gauge the similarity in colour of some mimetic morphs of Papilio dardanus (race tibullus) to their models (or putative models) across the whole spectrum visible to their main predators, birds (important for assessing the mimetic status of the morphs and thus building a population model, done in Chapter 7).

- Determine whether or not the white and black hippocoonides morph has evolved from a yellow and black morph merely by the loss of the yellow pigment (important for understanding the evolution of the various morphs and the pressures which drove that evolution).

In order to achieve these aims, the investigation was split into several parts. Specimens were firstly photographed under UV light to look for areas of UV reflectivity (Experiment 3-1). Then the fluorescence of the yellow pigment was quantified, and the other pigments checked for fluorescence with a fluorescence spectrometer (Experiment 3-2). In Experiment 3-3, the reflectance spectra of the pigments were measured using a spectrophotometer with an integrating sphere attachment. The structure of the scales was then investigated using both scanning (Experiment 3-4) and transmission (Experiment 3-5) electron microscopes and measurements of the lamellae and air spaces used to model the structural colours of the scales. Experiment 3-6 attempted to measure the colour reception capabilities of the butterflies in order to assess how they might see the spectra measured. Finally, Experiment 3-7 investigated the fading process which causes the yellow pigment in Papilio dardanus to become more orange with exposure to light. 


\section{Experiment 3-1 Photography under UltraViolet light}

\section{Introduction}

This first part of the investigation of the visual signals presented by the butterflies is designed to determine the patterns present on the upper surfaces of the wings. It concentrates on determining whether there are any areas of the wings which reflect UV light, since in many species these are reported to have little or no correlation with the Visible pigmentation patterns (Silberglied, 1979). Hence there may be patterns, caused by UV reflectance, which are not visible to the human eye.

Cockayne (1924, p7) described the appearance of Papilio dardanus and its morphs under UV light, and reported that the yellow pigment in males and trimeni females fluoresced blue-green, that the white areas of hippocoonides fluoresced violet, and the other colours showed no fluorescence. The yellow pigment in the males has subsequently been identified and named as Papiliochrome II (Umebachi 1961). Cockayne does not report in any more detail the appearance of the different forms under UV light, and he did not photograph the specimens in order to assess UV reflection.

Since our own eyes cannot see into the UV region of the spectrum we are unable to assess whether or not there is a UV component to any coloration. However, monochrome film is sensitive to UV wavelengths, and hence by photographing a specimen lit purely by UV light using monochrome film it is possible to see which patches reflect UV light as they show up as bright areas on the print. Normal glass photographic lenses may filter out some UV light, but in view of the considerable cost of UV-transparent plastic lenses, the experiment was carried out using a glass lens. Since some UV does pass through the lens, long exposure times were used to compensate for the degree of filtering.

\section{Aim}

The aim of this experiment is to determine whether or not there are UV reflecting scales present in Papilio dardanus, and if so whether or not they form patterns other than those seen in the Visible spectrum. 


\section{Method and Materials}

Dead specimens of Papilio dardanus collected by Cook et al. (1994) from the island of Pemba, Tanzania, were used in this study. The specimens had been kept in dark conditions, protected from sunlight. Cook et al. describe this population of race tibullus as having three female morphs (see Figure 31): the yellow and black 'male-like female' trimeni, the black and white Batesian mimic of Amauris niavius (Trimen, 1868), hippocoonides, and the rare orange-tinged lamborni (also known as trophonius). Within the classification of lamborni there are

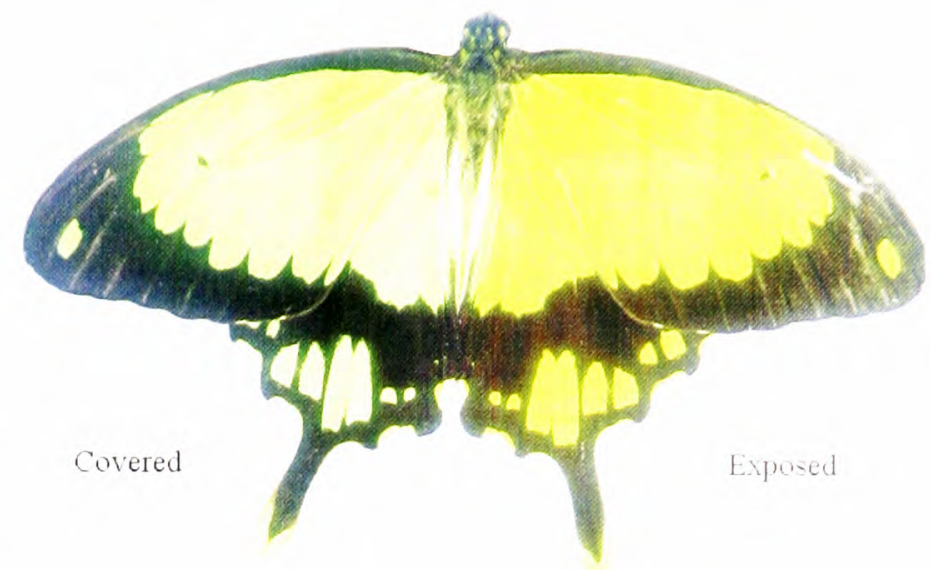

Figure 3-3 Demonstration of the fading of yellow pigment in Papilio dardanus by sunlight. Only the right wing was exposed. See Experiment 3-7 for a further investigation of this phenomenon.

two types - those which are similar to an orange-tinged trimeni ('yellow lamborni', or prototrophonius), and those similar to hippocoonides ('white lamborni', or trophonius) which is even rarer. In some races trophonius is a convincing mimic of Danaus chrysippus (Trimen, 1868) which is present on Pemba but on the island it is debatable whether the morph is a mimic or not. In addition, Cook et al. reported that the yellow pigment seen in the males and trimeni females, Papiliochrome II (Umebachi 1961), fades in sunlight to an orange colour (see Figure 3-3), and these 'faded' specimens were also studied.

A Canon EOS 650 camera with a 55-200mm Sigma lens was set up on a tripod in a dark room, loaded with Kodak T-Max 3200 black and white film. The camera was mounted directly above the specimens, and photographs were taken of the butterflies placed on black velvet under three different lighting conditions: a fluorescent tube lighting the specimen from directly above, $254 \mathrm{~nm}$ wavelength UV, and $356 \mathrm{~nm}$ wavelength UV light, both supplied by a UV fluorescent tube with two alternative wavelength outputs placed adjacent to camera, illuminating the specimens from above.

Photographs of the morphs and of Amauris niavius and Danaus chrysippus taken against a black background were also captured in 32 bit colour using a Silicon Graphics IndyCam ${ }^{\text {TM }}$ digital camera (Model CMB006C) under fluorescent tubes (illuminating from above) and 356nm UV light (placed above the specimens as before). Both the upper wing surfaces and the undersides of the wings of the Papilio dardanus specimens were photographed. 


\section{Results}

1) Using monochrome film

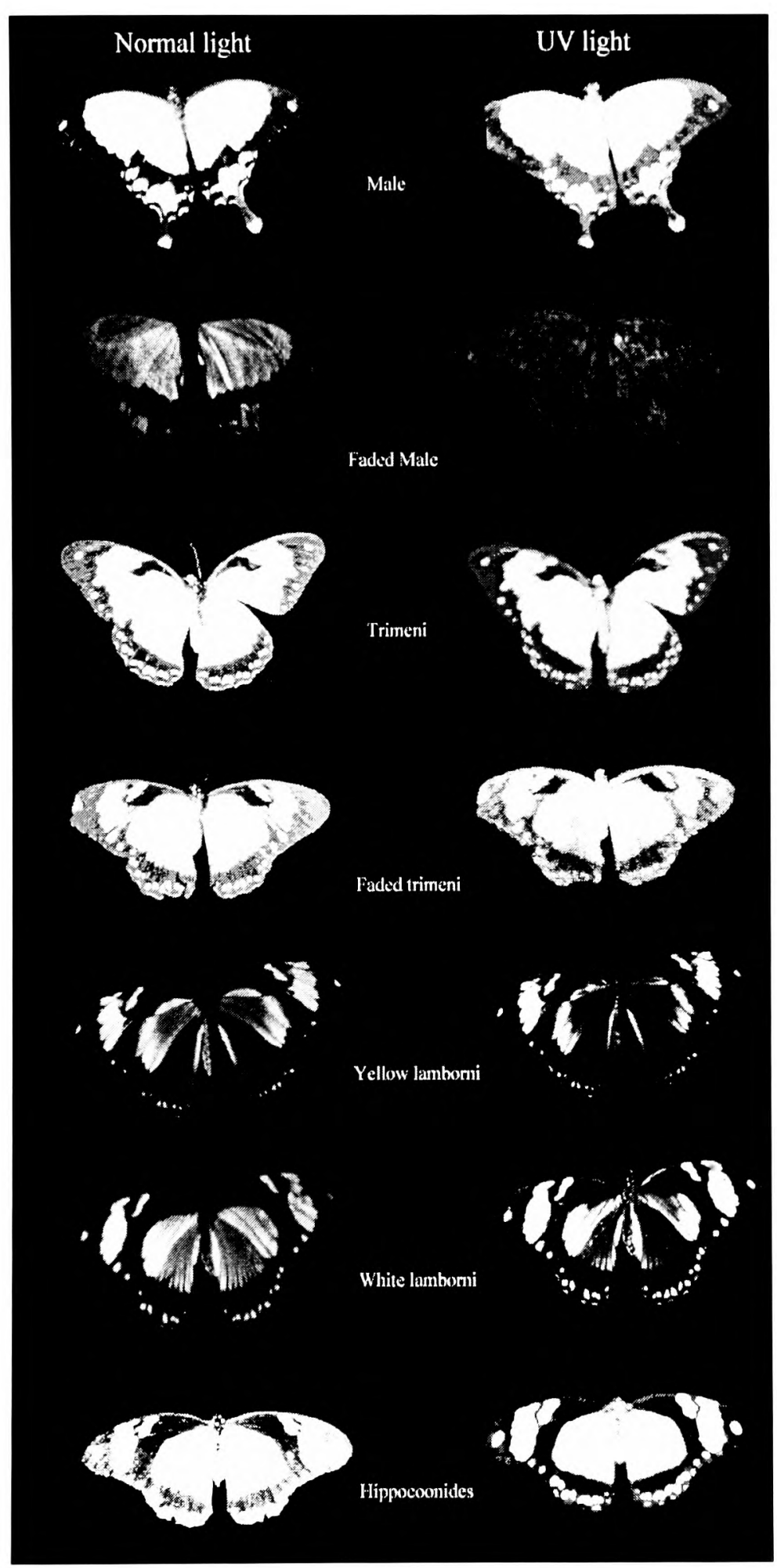

Figure 3-4 Photographs of the different morphs of Papilio dardanus taken under normal and UV $(356 \mathrm{~nm})$ light using monochrome film.
The photographs taken under $254 \mathrm{~nm}$ light were identical to those taken under $356 \mathrm{~nm}$ light except that the reflectance from them showed less intensely on the film. Only those under $356 \mathrm{~nm}$ light are shown in Figure 3-4.

The yellow pigment of the males (Papiliochrome II) fluoresced a bright greenish yellow under both wavelengths of UV light, showing the same pattern as when illuminated with normal light. The tips of the tails, which are slightly orange coloured, did not fluoresce. Essentially the unfaded males looked very similar under the two light wavelengths.

The orange, faded males looked very different under UV light, hardly fluorescing at all, and gaining a 'speckled' appearance.

The obvious black markings also faded, becoming hardly visible.

The trimeni females fluoresced similarly, although the forewings did not appear as bright. The black 'epaulette' markings near the base of the 
leading edge of the front wings became particularly obvious. It may be of significance that these epaulettes are present in the tailed, andromorphic females (but not the males) of races meriones, humbloti, and antinorii (Trimen, 1868).

The faded trimeni females lost a lot of their fluorescence. The black markings became less obvious, except for the epaulettes which remained dark. Around them some fluorescence remained, providing quite a lot of contrast, and the veins (particularly those on the hind wings) also fluoresced, unlike those on any of the other morphs (including the faded male). This could be due to scales around the veins being slightly more protected from damage than the others on the wing.

The yellow lamborni females appear to have the same Papiliochrome II pigment and markings as trimeni females. They also have a bright orange pigment on the hind wings and the lower fore wings. This orange pigment absorbs UV light, appearing as dark as the black markings in the photographs.

The white lamborni females have white patches in the place of the yellow on the yellow lambornis, and the black epaulette markings are more continuous with the wing edgings and sharply defined as in hippocoonides. The orange pigment is slightly paler than that in yellow lamborni, and the white patches have a pale pinkish sheen to them. Under UV light the orange pigment absorbs (although possibly not as much as that in yellow lamborni), and the white scales were not observed to fluoresce as described by Cockayne (1924). However, on the film, the white patches appear as bright as the yellow pigment on the other morphs, indicating that it is reflecting, but in the UV spectrum where it could not be observed. Another piece of evidence that these bright regions are due to UV reflectance is the blur on their edges - particularly noticeable on the image of hippocoonides (see Figure 3-4). This is due to the fact that the camera was focused using Visible light, whilst UV light has a shorter wavelength. It is possible that the UV light which Cockayne used included some visible wavelengths, and it was these violet wavelengths being reflected by the white patches that he described as violet fluorescence. He describes this violet fluorescence in a lot of species with white markings, and it seems likely that these are in fact reflective to all wavelengths rather than being fluorescent.

Hippocoonides females have similar markings to the other females, although the black patterning tends to be less variable, and resembles that of their model, Amauris niavius. The background colour is white, with a pinkish sheen (as is Amauris niavius). Under UV light neither the model (see Figure 3-5) nor the females themselves appear to fluoresce, but on film the white appears bright, again suggesting that the scales reflect UV light. 


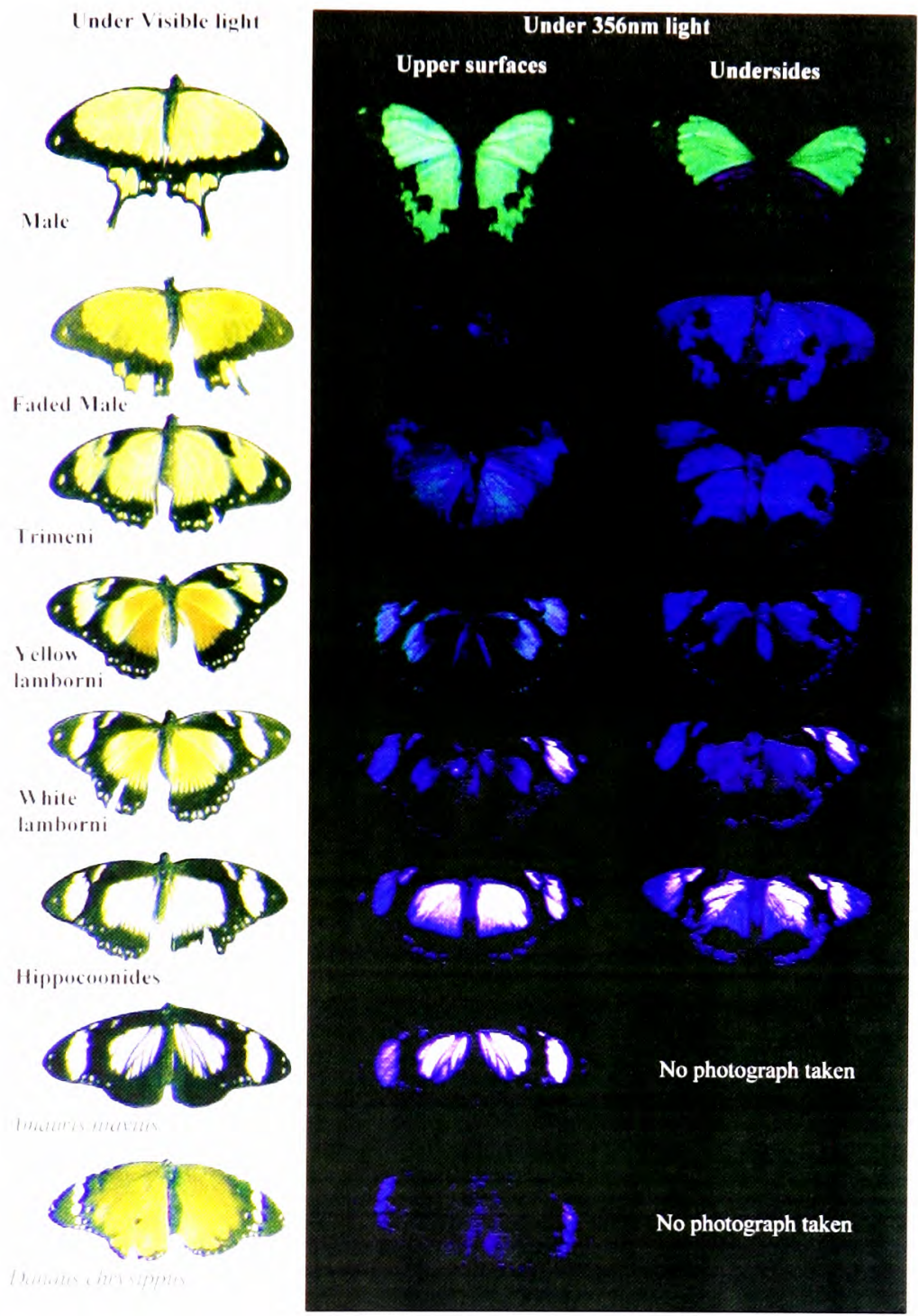

Figure 3-5 Colour digital photographs of the different morphs of Papilio dardanus and its models taken under fluorescent strip lighting and UV (356nm) light.
2) Using a digital camera

Before undertaking this experiment, it was not known whether or not the digital camera would be sensitive to UV light, but the images obtained (see Figure 3$5)$ show the white patches on hippocoonides and white lamborni even more strongly than the yellowish fluorescence from Papiliochrome II. All the photographs taken under UV light have had their luminescence increased by $200 \%$ (using Paint Shop Pro version 4 on a PC). As before, the white patches did not fluoresce visibly, and so the camera must have been sensitive to some UV light reflected, and displays this as a purple coloration. These images are probably similar to what Cockayne (1924) observed. It is noticeable, however, that the purple coloration

of the white patches under UV light is 'shiny', rather than being of a uniform colour and intensity as seen in the fluorescence of the male. This indicates that the coloration observed is due to reflection rather than fluorescence. Experiment 3-2 should confirm whether or not this is indeed reflection rather than fluorescence. It is also interesting that the trimeni female appeared not to fluoresce as strongly as the male. Cockayne $(1924, \mathrm{p} 7)$ described the trimeni females as fluorescing "with the same brilliant blue-green colour as the male" so it could be that this particular individual was beginning to fade and lose fluorescence. This is investigated further in Experiment 3-2.

The photographs of the undersides of the wings reveal that white patches on the underside of the wings of hippocoonides reflect UV just as those on the upper surfaces do. The same is true of the white patches on the undersides of the forewings of the white lamborni morph. Both the yellow patches on the forewings and the orange cryptic hindwings of trimeni and yellow lamborni, appear to reflect the UV but not to fluoresce. The faded male also appears to show UV-reflecting hindwings, 
whilst the unfaded male appears very different - with the yellow forewings fluorescing and the hindwings neither fluorescing or reflecting UV. However, photographs taken of other male specimens (at slightly different angles of incidence of light due to the fact that the specimens were not entirely flat, and inclined to rock slightly when placed on their upper surfaces) reveal that sometimes reflectance is observed on both fore and hindwings, and sometimes only fluorescence on the forewings is seen(see Figure 3-6). This is probably due to the fact that the reflectance is only visible at some angles, and that at some angles its presence on the forewings makes the fluorescence invisible. This suggests that the fluorescence is much less intense than the reflectance, which will be tested in Experiment 3-3. It is interesting to note that although the yellowy orange undersides of the hindwings of the males and trimeni females do not fluoresce, they do reflect UV light. This will alter their appearance to predators with UV vision compared with how we perceive them.

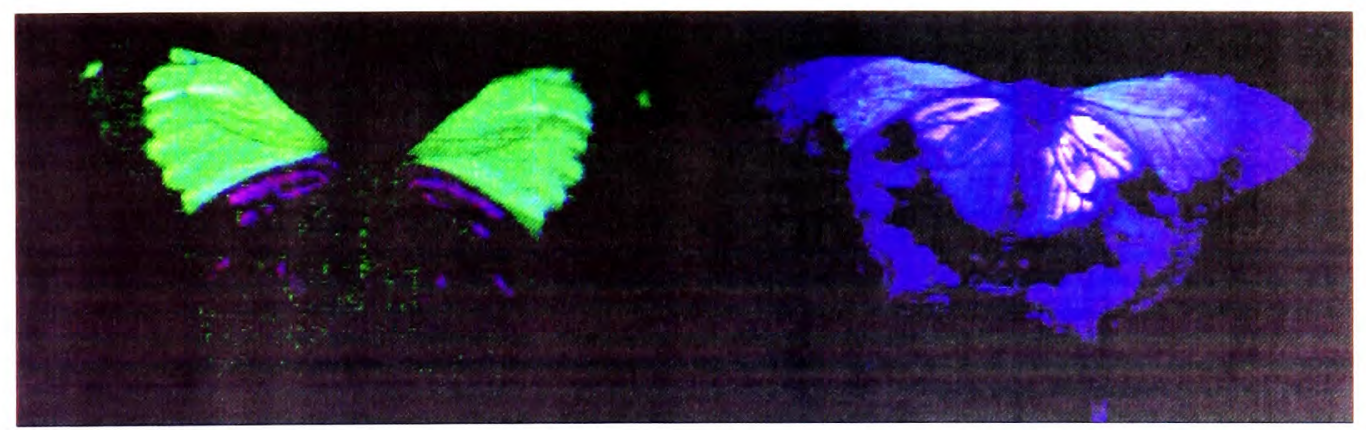

Figure 3-6 The undersides of two male Papilio dardanus photographed under $365 \mathrm{~nm}$ light at two angles (left - normal to surface, right - slightly oblique).

\section{Discussion}

It can be seen that the white scales in hippocoonides and white lamborni females reflect UV light and that the Papiliochrome II pigment found in males, which fluoresces a greenish yellow under UV light, also appears to be found in both trimeni females and yellow lamborni females. The orange and black pigments do not fluoresce and the scales do not reflect UV light. It is difficult to tell whether or not the scales containing the yellow fluorescent pigment also reflect UV light, since such a reflectance might be masked in the pictures by the fluorescence. The monochrome film only records the intensity and not the wavelength of the light involved. In the colour photographs, the fluorescent patches appear yellow when the photograph is taken from directly above. However, the evidence from the photographs of the undersides of the wings taken at a slightly oblique angle indicates that directional UV reflectance may be present in the yellow patches, but merely masked at that particular angle by the fluorescence. Experiment 3-3 investigates the presence of UV reflectance further. 


\begin{tabular}{|c|c|c|c|}
\hline 'Human colour' & Morphs found in & UV reflectant? & Fluorescent under UV? \\
\hline Black & All & No & No \\
\hline White & $\begin{array}{c}\text { Hippocoonides, White } \\
\text { lamborni }\end{array}$ & Yes & Yes \\
\hline Yellow & $\begin{array}{c}\text { Male, Trimeni, Yellow } \\
\text { lamborni }\end{array}$ & Probably & No \\
\hline Bright Orange & $\begin{array}{c}\text { White lamborni, } \\
\text { Yellow lamborni }\end{array}$ & No & No \\
Yellowy orange & $\begin{array}{c}\text { Underside of } \\
\text { hippocoonides }\end{array}$ & Yes & \\
\hline
\end{tabular}

Table 3-1 Summary of the properties of the colours of the different morphs of Papilio dardanus investigated

Given that the UV reflectance is almost certainly achieved by structural means, from these pictures it would be predicted that the scales in the black and orange areas would have a different structure from those in white areas, lacking reflective structures. It is not possible to predict from these pictures whether or not the scales containing yellow pigment also possess UV reflective lamellae.

These photographs also indicate that there are no patterns due to UV reflectance which are uncorrelated with the Visible patternings. This means that the butterflies should appear similar in patterning to each other and to their predators as they do to us, but that their coloration is likely to be seen very much differently due to the presence of UV reflectance which is invisible to humans. Experiment 3-3 quantifies the colours used by these forms, and Experiment 3-6 quantifies the visual sensitivities of the butterflies in order to assess how the colours might appear to them.

\section{Experiment 3-2 Analysis of the pigments using a Fluorescence Spectrometer}

\section{Introduction}

Since Papiliochrome II, the yellow pigment in Papilio dardanus, fluoresces in the Visible spectrum under UV light, any spectra produced of this pigment must take this into account as in daylight, the fluorescence will add to the colour produced by the yellow patches. For example, if a spectrophotometer emits each wavelength of light separately and then measures only how much of that particular wavelength returns from the wing surface, this fluorescent component will be missed. In order to add the component back in, therefore, it is necessary to measure the wavelength emitted and its intensity at each wavelength of incident UV light. This can be achieved using a fluorescence spectrometer, which, unlike a traditional spectrometer, has two monochromators - one to produce the 
incident beam and the second to monochromate the reflected light. Thus the wavelengths of emitted light are recorded as well as their intensity.

This method should also clarify whether or not the violet 'fluorescence' recorded by Cockayne (1924) as being emitted from the white patches in hippocoonides under UV light was fluorescence or reflectance of wavelengths in the Visible region of the spectrum not filtered out by his apparatus.

\section{Aim}

The aim of this experiment is to determine the wavelengths of light which produce fluorescence in Papiliochrome II, and both the wavelengths and approximate intensities of the fluorescence produced in order to assess the impact of the fluorescence on the overall reflectance spectrum of the coloured patches. It is also the aim to determine whether or not the white patches in hippocoonides fluoresce.

\section{Method and Materials}

A Perkin Elmer LS50 fluorescence spectrometer was set up so as to allow a mounted butterfly to be placed into the excitation beam, and a spectrum of the resulting scattered light to be recorded and plotted on a PC. Whole specimens were pinned onto non-reflective, matt black polystyrene, and then placed so that the beam of incident light formed a focused band (approximately $1 \mathrm{~mm} \mathrm{x} 1 \mathrm{~cm}$ ) on the appropriate area of the wing. Two types of scan were used:

1. Emission scan: The excitation wavelength applied to the pigment is fixed, and a plot is drawn of the intensity of light of different wavelengths being emitted. In the case of pure reflection, peaks will appear only at the wavelength applied and its subharmonics (at double the wavelength etc.). Where fluorescence is also occurring, lower, more spread out peaks will be seen at longer wavelengths (not at shorter wavelengths, as this would require more energy than is applied).

2. Excitation scan: The excitation wavelength is varied, and a plot is drawn of the resulting changes in intensity of the emission at a particular wavelength. This can be used to determine the excitation wavelength which causes the maximum fluorescence, and check whether one or more pigments are fluorescing (causing more than one peak in the excitation plot).

Emission scans were performed on the main pigments in each morph, and also the black from a male. Where a pigment was found to fluoresce, an excitation scan was done at several wavelengths throughout the fluorescence band. 


\section{Results}

1) Emission scans (see Figure 3-7 and Figure 3-8).

Emission scans

a)

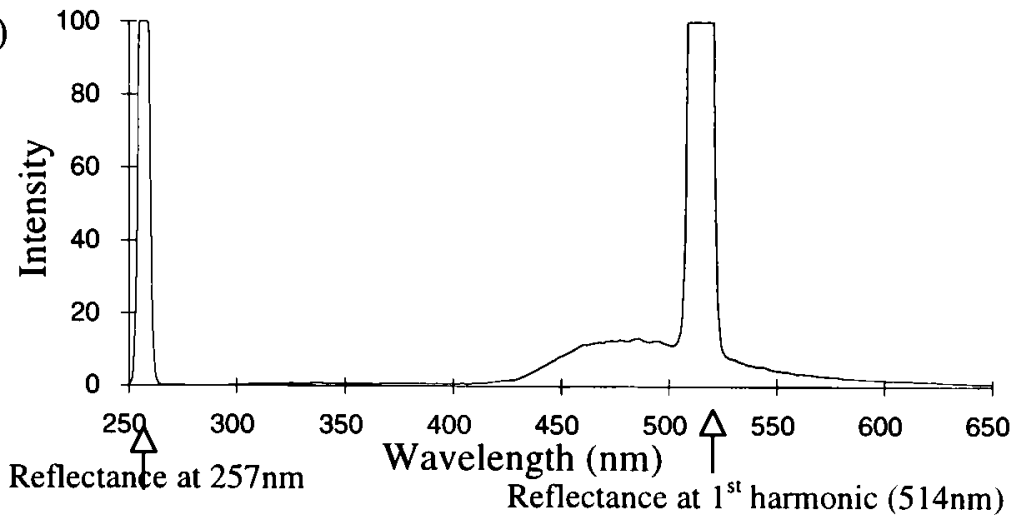

b)

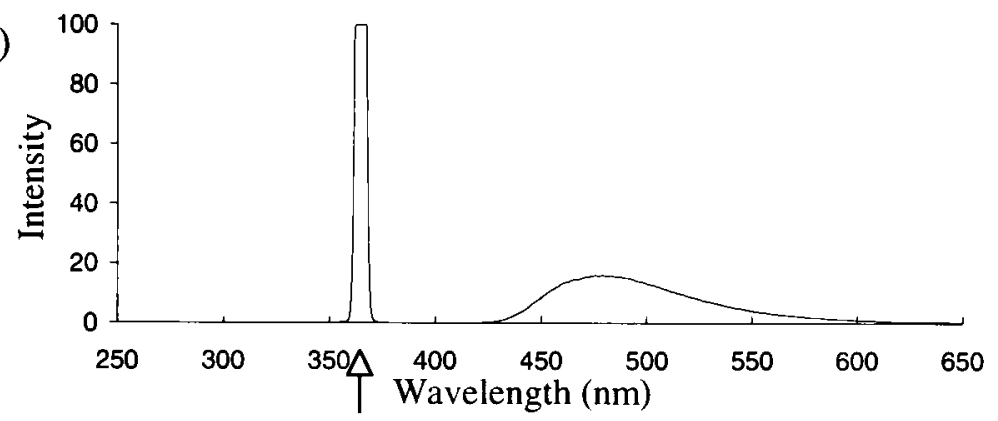

Reflectance at $356 \mathrm{~nm}$
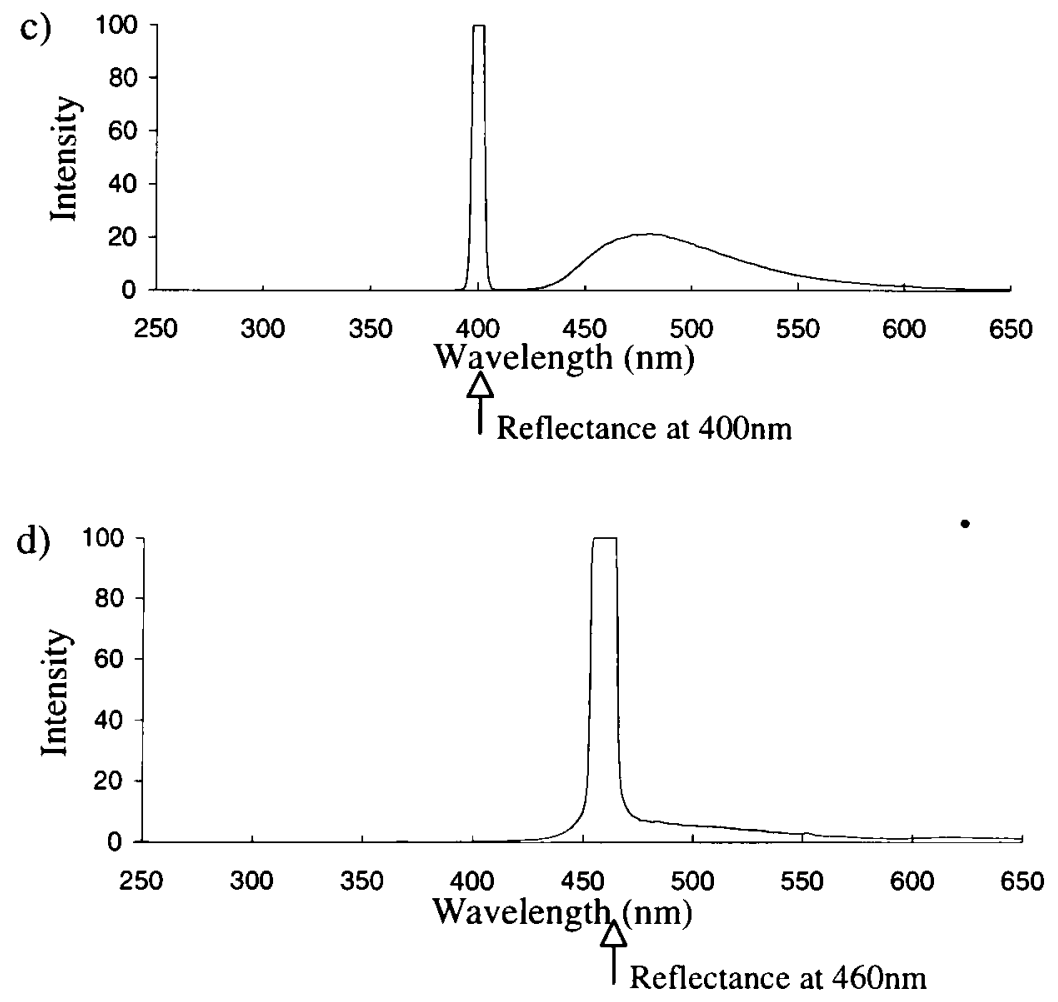

Figure 3-7 Emission scans of yellow pigments found in Papilio dardanus: Intensity of response at various wavelengths when illuminated with a) $257 \mathrm{~nm}$, b) $356 \mathrm{~nm}$, c) $400 \mathrm{~nm}$, d) $460 \mathrm{~nm}$ of the Papiliochrome II pigment found in males were done at $257 \mathrm{~nm}$, $356 \mathrm{~nm}, 400 \mathrm{~nm}$, and $460 \mathrm{~nm}$. At all these wavelengths a broad fluorescence band was seen between $425 \mathrm{~nm}$ and $630 \mathrm{~nm}$, with a peak at $483 \mathrm{~nm}$. In each graph the high peak is the scatter from the wavelength applied or its $1^{\text {st }}$ harmonic (at double the wavelength).

The same scans were done on the yellow pigment in trimeni and yellow lamborni females, and the same fluorescence was seen, and at the same intensity. 
Emission scans at

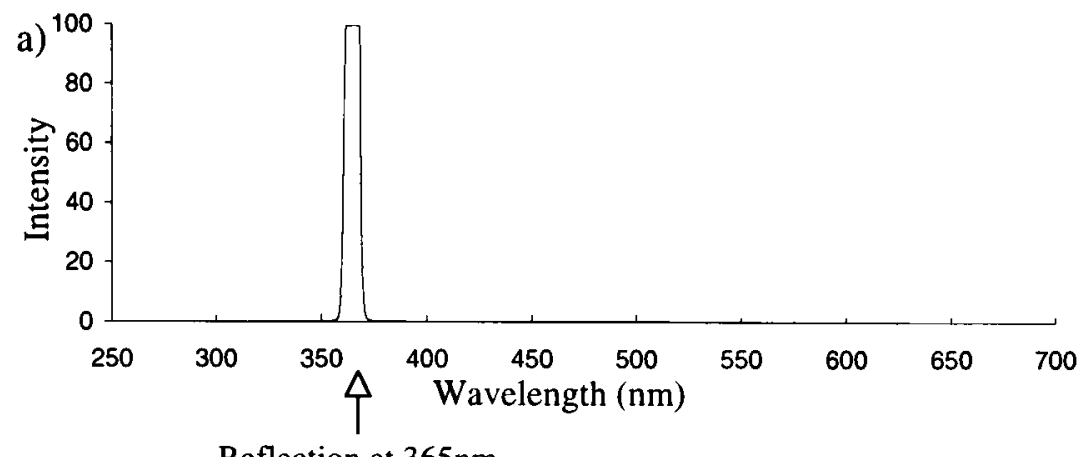

Reflection at $365 \mathrm{~nm}$

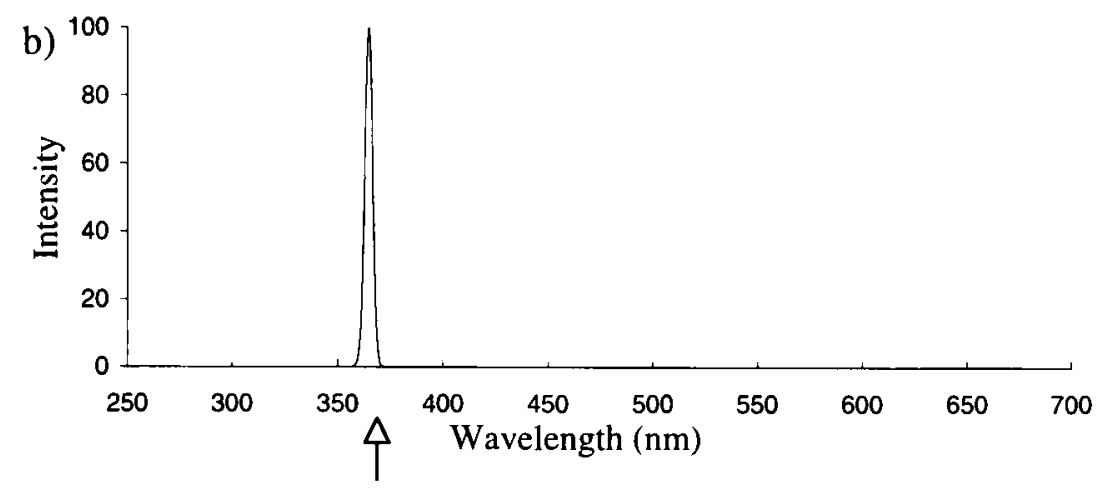

Reflection at $365 \mathrm{~nm}$

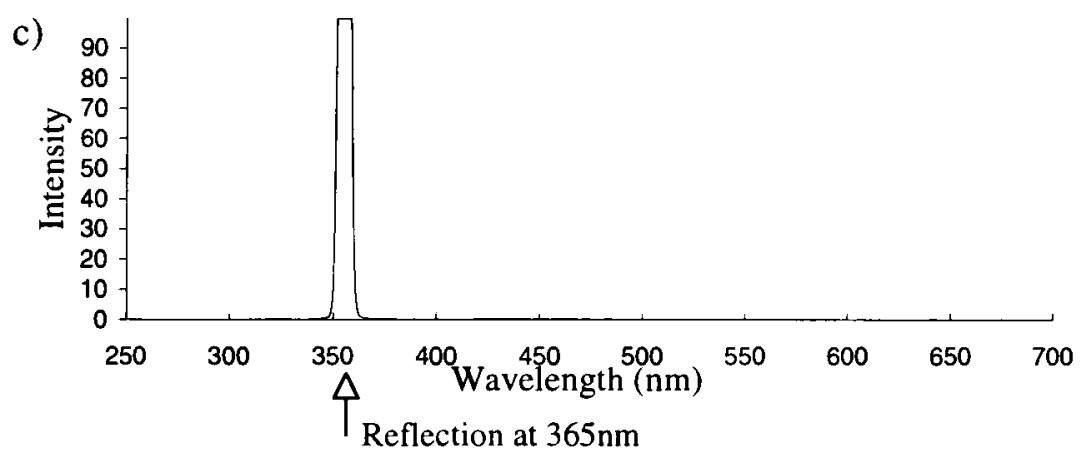

Figure 3-8 Emission scans of a) faded yellow, b) orange, and c) white pigments found in Papilio dardanus illuminated with $365 \mathrm{~nm}$ light. the same wavelengths of the sunlight-faded

Papiliochrome II of males showed no fluorescence at all, the only peaks being those due to scatter of the incident light (Figure 3-8a).

The orange pigment in yellow lamborni showed no fluorescence (Figure 3$8 b)$. There is also a suggestion that it reflects this wavelength less well as the reflectance peak appears not to be as large, but this is investigated fully in Experiment 3-3.

Emission scans at the same wavelengths of the white scales in hippocoonides also showed no fluorescence (Figure 3$8 \mathrm{c}$ ), and the same was true of its model, Amauris niavius.

2) Excitation scans (see Figure 3-9)

Excitation scans of the yellow pigment showed that at the peak wavelength of fluorescence $(483 \mathrm{~nm})$ the excitation wavelengths formed a smooth, shallow curve from $340 \mathrm{~nm}$ to a peak at around $400 \mathrm{~nm}$ and then dropped off more rapidly from 420 to $440 \mathrm{~nm}$. 


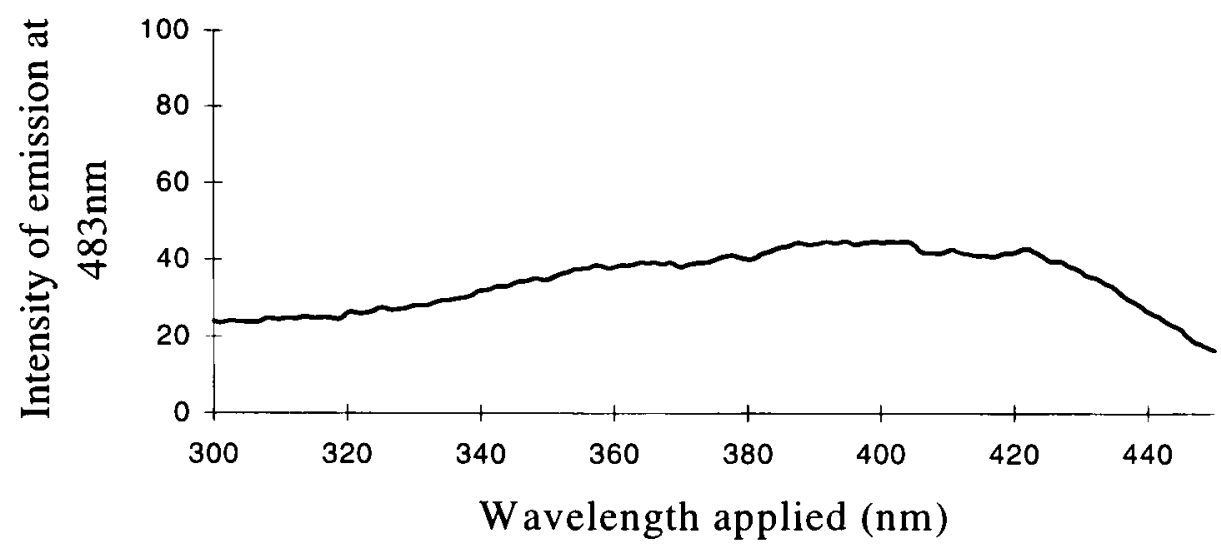

Figure 3-9 Excitation scan of yellow pigment found in Papilio dardanus.

\section{Discussion}

The fluorescence spectra of the yellow pigment were found to be the same in males and the trimeni and yellow lamborni females. This pigment, Papiliochrome 11 (Umebachi, 1961) is reported to have a peak of absorption at $380 \mathrm{~nm}$ (Umebachi \& Yoshida, 1970). Here it is found to fluoresce at a range of wavelengths around $483 \mathrm{~nm}$ when exposed to UV light of wavelengths between $340 \mathrm{~nm}$ and $400 \mathrm{~nm}$ (the wavelengths which it absorbs most readily). The fluorescence is, however, very weak when compared with the reflectance of the wings (seen in Figure 3-7), so the fluorescence of the pigment will have a negligible effect on the overall colour of the yellow wing patches. This fluorescence is lost in older specimens where the yellow pigment has faded to orange, but the weakness of the fluorescence means that this loss will not significantly contribute to the colour change of the patches. The breakdown of Papiliochrome II in sunlight is examined in greater detail in Experiment 3-7.

The white patches of hippocoonides were found not to fluoresce when exposed to UV light of any wavelength. Thus it can be concluded that the violet 'fluorescence' reported by Cockayne (1924), and the colours noted in Experiment 3-1 were due to reflectance of the incident light rather than fluorescence of a pigment.

\section{Experiment 3-3 Reflectance spectra of the pigments}

\section{Introduction}

Reflectance spectra are an objective measurement of the colour of an object. This is very important in the study of visual signalling as different species have different spectral sensitivities. For example, two butterflies may be indistinguishable to humans, but easily separable by animals which see in the UV region of the spectrum due to a UV component in the colour of one of the butterflies. 
Measuring the reflectance spectra of the colours allows humans to measure exactly what visual signals are being produced and received by the butterflies, and thus to assess the similarity of species, especially in the case of comparing potential model and mimic pairs. As discussed in the introduction to this chapter, the directionality of structural colours in butterflies complicates the process of measurement. Simply measuring the reflectance at one angle relative to the angle of illumination can misrepresent the total reflectivity of the scales, as their structure reflects different wavelengths at different angles relative to the illumination. Although it is technically possible to measure the reflectance from the wing at every angle, this is very laborious and produces a very large amount of data which is difficult to interpret. In this case the problem may be simplified by taking measurements using an integrating sphere attachment to the spectrophotometer, which collects reflected light from every angle and records only the total reflectance. Microscopy of the scales can reveal how the structural colours are formed, and some of these methods can be modelled mathematically to predict the exact form of the structural colours.

\section{Aim}

The aim of this experiment is to achieve an objective measurement of the coloration of the various morphs of Papilio dardanus in order to assess how they might appear to each other and to their predators.

\section{Method and Materials}

A Shimadzu UV- 2100 Spectrophotometer with integrating sphere attachment was used to measure the reflectance spectra of the colours. The wing samples were held against the $18 \mathrm{~mm}$ diameter circular sample hole in the integrating sphere by a matt black, spring-loaded mount. The central area of the sample $(5.5 \mathrm{~mm} \times 3 \mathrm{~mm})$ is used.

Two beams enter the sphere alternately, one from either side. One is directed at a totally reflective $\mathrm{BaSO}_{4}$ control, the other at the sample. The beams hit the surfaces at $8^{\circ}$ to the normal, and all the scattered light is then gathered by the internally reflective sphere and measured by a photomultiplier at the base. The intensity of the reflection of the sample is plotted at each wavelength as a percentage relative to the reflectance of the control.

The spectrophotometer measures the reflectance at each wavelength in turn, at $0.5 \mathrm{~nm}$ intervals, from $240 \mathrm{~nm}$ to $800 \mathrm{~nm}$. A different bulb is used from $360 \mathrm{~nm}$ downwards into the UV, sometimes causing a slight discontinuity in the spectrum at $360 \mathrm{~nm}$. The results are plotted on a PC, and can then be exported in ASCII format to a spreadsheet for analysis.

Scans were done of the yellow coloration on the upper surface of the forewing, lower surface of the forewing, and upper surface of the hindwing of three male specimens of races tibullus, cenea, 
humbloti, and one male of meriones of Papilio dardanus. This allows a comparison of the different areas of the wing, all of which are thought to contain the same pigment, but which might have differing structural colours. It also compares the different geographic races of the species (See Appendix 1 for a summary of the races). Little is known about the inter-relatedness of the races, and particularly it is not clear which most represents the primitive condition (either the polymorphic land races, or the monomorphic island races meriones and humbloti). Any racial differences in the colour may therefore be phylogenetically useful.

Scans were next done of the yellow coloration on the upper forewing, lower forewing, and upper hindwing of three specimens of the trimeni female morph from race tibullus, and a female specimen of race meriones of Papilio dardanus. Any differences between the spectra of the andromorphic females, and between them and the males may shed light on whether the tailed females in meriones are ancestral or derived, and how closely the tail-less andromorphic females resemble the males in colour.

Scans were next done on the yellow patches on the upper forewings of three specimens of the yellow lamborni female morph from the tibullus race of Papilio dardanus. It is likely that this morph has evolved from the trimeni morph, and if this is so, the colour of the yellow patches on the wings should match that of the trimeni females.

To contrast these results, scans were done on the upper forewing of three specimens of Mylothris ngaziya, a butterfly from Grande Comore. This butterfly appears, to human observers, very similarly coloured to the Papilio dardanus of race humbloti that fly in the same areas. Since Mylothris ngaziya is generally considered unpalatable (since Carpenter, 1941), it is possible that the Papilio dardanus may be gaining some protection from mimicry of it. These scans should reveal whether or not the two species have genuinely similar spectral colours.

Scans were made of the upper surface of the forewings of three old and faded specimens of the female morph trimeni and one faded male from the tibullus race of Papilio dardanus. The colour change of the yellow patches reported by Cook et al. (1994) has not been quantified before, so these scans should reveal exactly what changes are taking place.

The spectra were obtained for the orange pigment from the upper surfaces of the hindwings of three specimens of each of the white and yellow lamborni females from the tibullus race of Papilio dardanus, and also for the orange pigment from one specimen of Danaus chrysippus. Cook et al. (1994) hypothesised that the lamborni females could be mimicking the old, faded males (which appear orange) in order to avoid sexual harassment from males, whilst others (originally from Trimen, 1868) 
conclude that lamborni is a mimic of the butterfly Danaus chrysippus. The analysis of the spectra of the orange pigments may clarify this issue.

The white coloration on the upper surfaces of the forewings and hindwings of 3 specimens of the hippocoonides female morph from race tibullus and 2 specimens from race cenea of Papilio dardanus were analysed, and compared with the white pigment from the undersides of the hindwings of 3 specimens of hippocoonides from tibullus and 2 from cenea race of Papilio dardanus. This should give a cross-race comparison of any variation in colour, and also determine whether there is any difference in the colour of the upper and lower surfaces of the forewings. Scans were also done of the white patches on the forewings of the white lamborni morphs from race tibullus. Comparison of these with the white patches in hippocoonides may shed some light on whether the white lamborni morph is likely to have evolved from a hippocoonides morph through the addition of orange pigment to the hindwings, or from a trimeni morph via yellow lamborni.

To compare with the data from the hippocoonides females, scans were done on the white coloration of the upper surfaces of the forewings of 3 specimens of Amauris niavius, and the bottom surface of one of the specimens (the others were too delicate to allow this). Hippocoonides is generally regarded as a mimic of Amauris niavius (Trimen, 1868), and these scans should indicate whether the two truly resemble each other in colour as well as pattern.

\section{Results}

a) Spectra of yellow in males of race tibullus

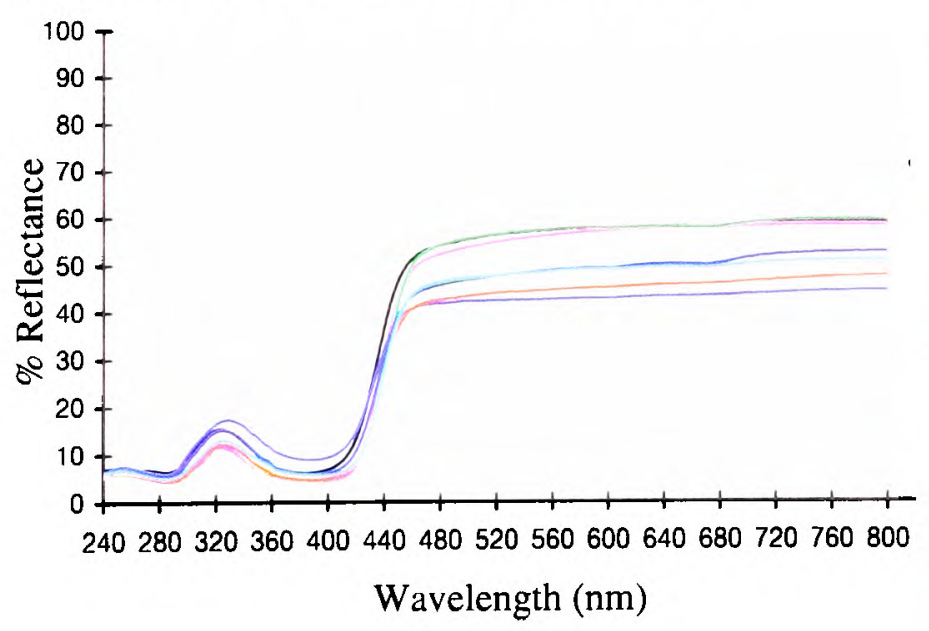

Lower surface forewing (Specimen 1)

Upper surface hindwing (Specimen 1)

Upper surface forewing (Specimen 1)

Lower surface forewing (Specimen 2)

Upper surface forewing (Specimen 2)

Upper surface hindwing (Specimen 2)

Lower surface forewing (Specimen 3)

Upper surface hindwing (Specimen 3)

Upper surface forewing (Specimen 3) 


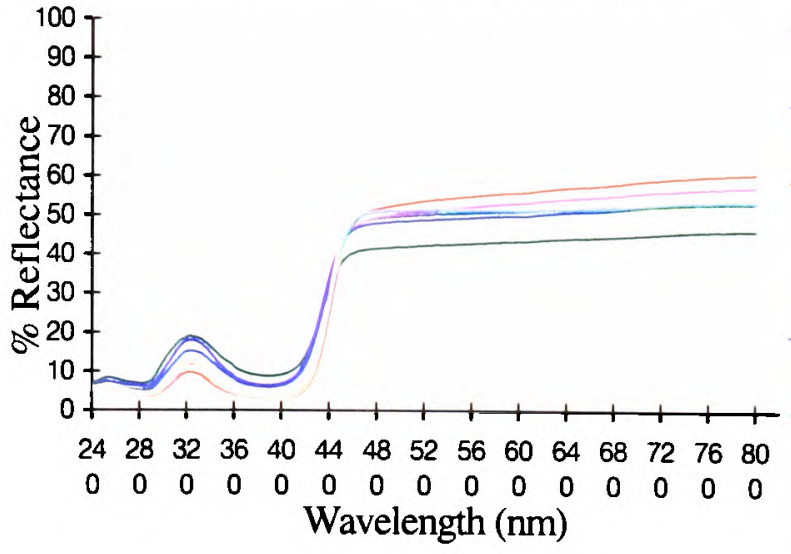

b) Spectra of yellow in males of race cenea

- Lower surface forewing
(Specimen 1)
- Upper surface hindwing
(Specimen 1)
- Lower surface forewing
(Specimen 2)
- Upper surface hindwing
(Specimen 2)
Upper surface forcwing
(Specimen 2)
- Lower surface forewing
(Specimen 3)
- Upper surface forewing
(Specimen 3)
Upper surface hindwing
(Specimen 3)

c) Spectra of yellow in race meriones

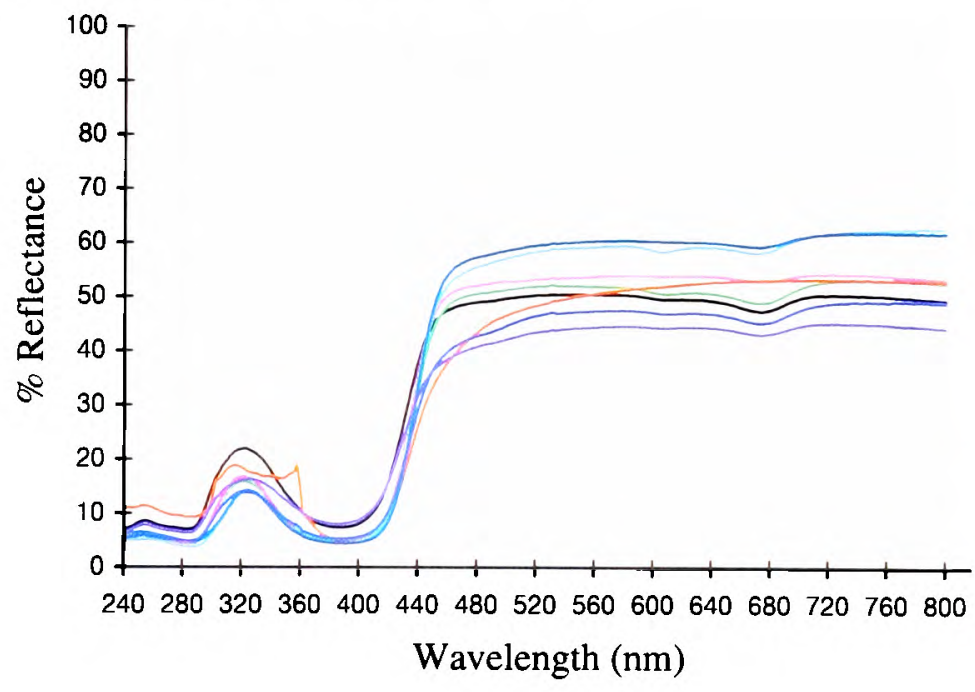

d) Spectra of yellow in race humbloti

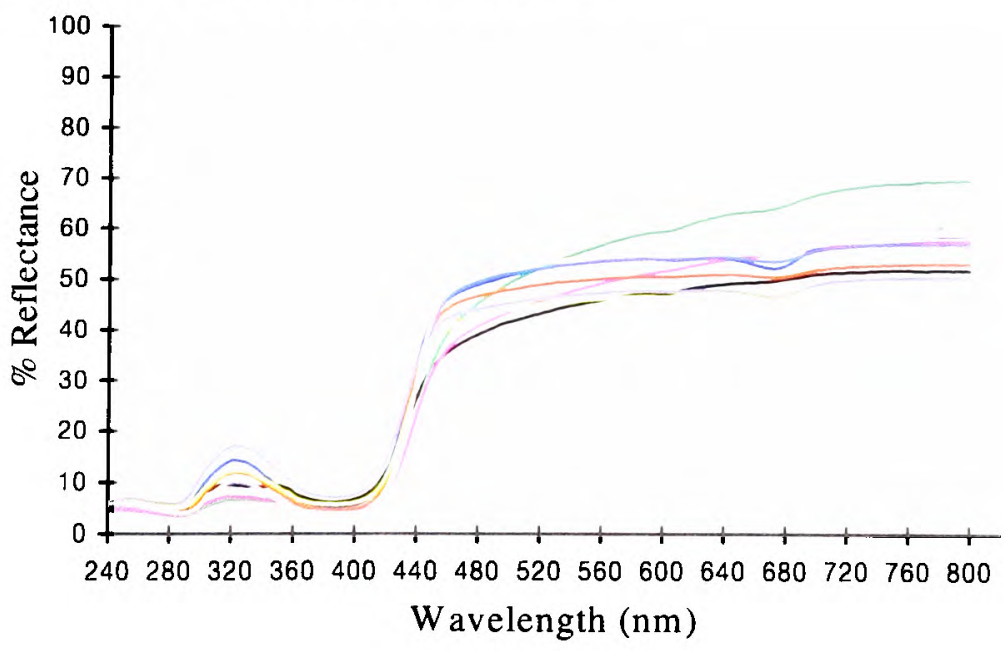

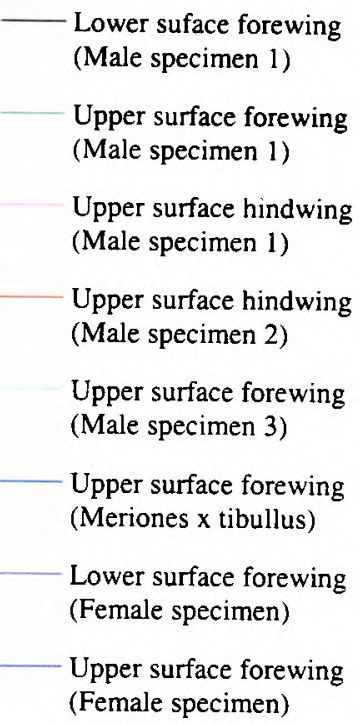

\footnotetext{
Lower surface forewing (Specimen 1)

Upper surface forewing (Specimen 1)

Upper surface hindwing (Specimen 1)

Lower surface forewing (Specimen 2)

Upper surface hindwing (Specimen 2)

Upper surface forewing (Specimen 2)

Lower surface forewing (Specimen 3)

Upper surface forewing (Specimen 3)

Upper surface hindwing (Specimen 3)
} 
e) Spectra of yellow in females of race tibullus
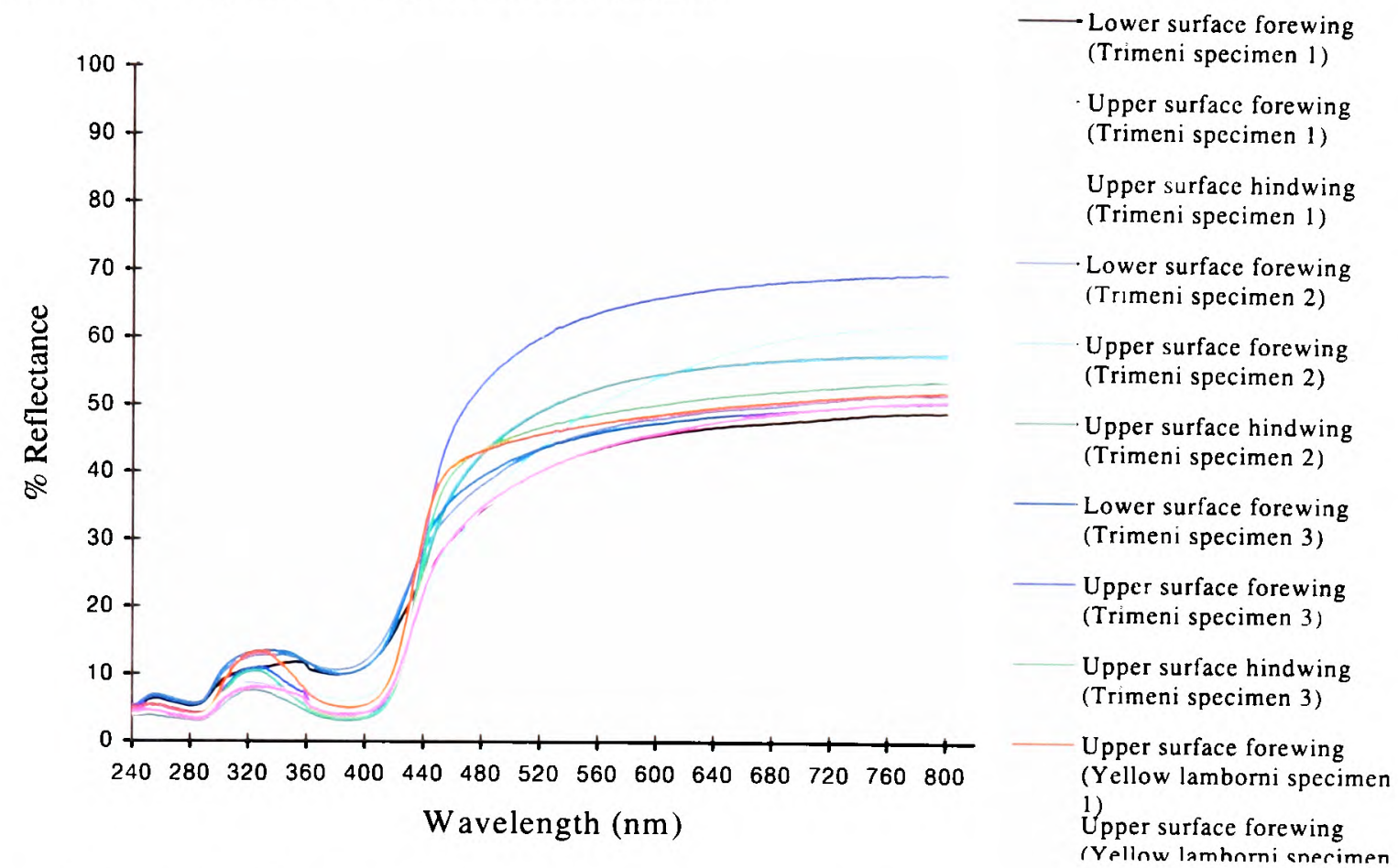

f) Spectra of yellow in Miliothrice ngazidja

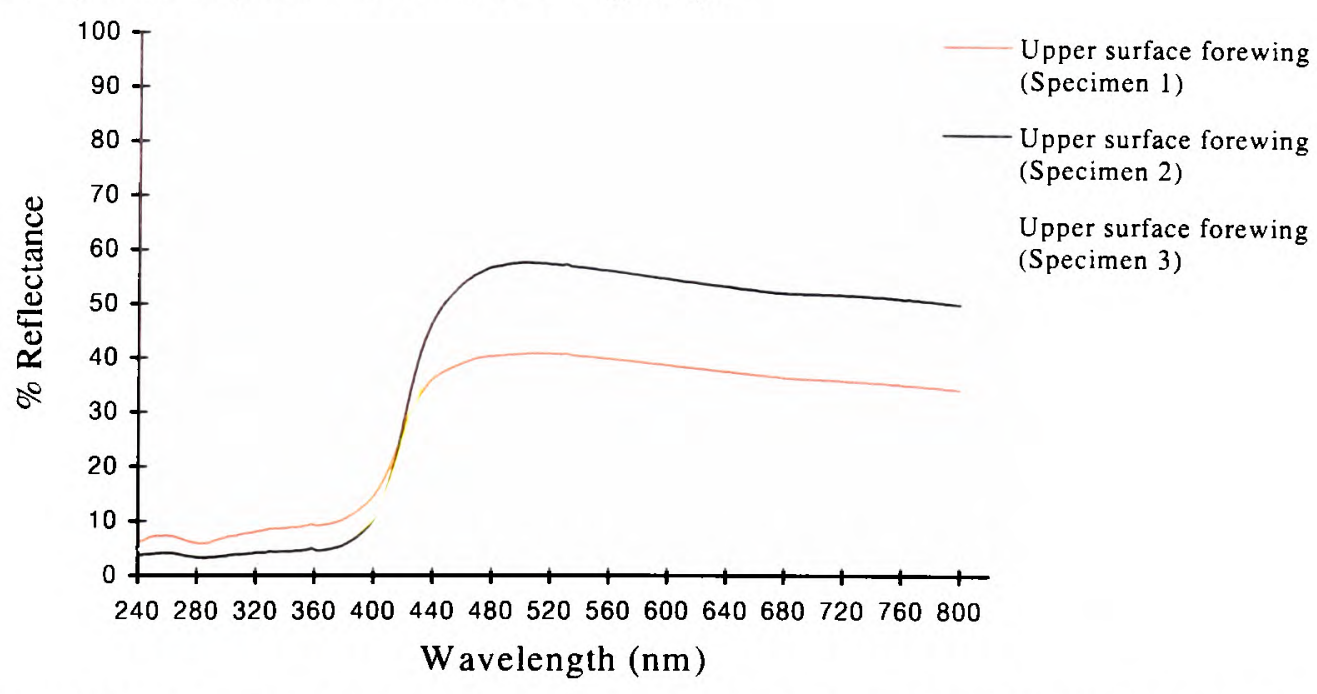

Figure 3-10 Reflectance spectra of yellow pigments found in Papilio dardanus: ${ }^{\circ}$ a) males, race tibullus, b) males, race cenea, c) both sexes, race meriones, d) males, race humbloti, e) females, race tibullus, f) Miliothris ngazidja for comparison.

The reflectance spectra of the yellow pigments in all males (Figure 3-10a, b, c, and d), trimeni females (Figure 3-10e, blue-green lines), and yellow lamborni females (Figure 3-10e, red-pink lines) of Papilio dardanus were all very similar. In each case the reflectance was high (around 50\%) from $800 \mathrm{~nm}$ to about $445 \mathrm{~nm}$ (red, yellow, and green). They all then show a sharp decrease in reflectance (to under 10\%) over the blue/violet region of the spectrum until about $370 \mathrm{~nm}$, where the reflectance slowly increases to a small peak (under $20 \%$ reflectance) at $320 \mathrm{~nm}$ in the UV region. The reflectance then returns to under $10 \%$ from about $290 \mathrm{~nm}$ onwards (a slight fluctuation in the reflectance below this was also present in the control, empty, scan). The specimens (including the female) from the meriones (Figure 3-10c) and humbloti (Figure 3-10d) races show a slight dip in the reflectivity at 
about 680nm which was not seen in the races tibullus (Figure 3-10a) and cenea (Figure 3-10b). This might be a clue that these two races are closely related, and it is possible that an analysis of the pigments in the two sister species (Papilio constantinus and Papilio phorcas, Clarke et al., 1991; Vane-Wright \& Smith 1991) might indicate whether this spectral feature is primitive to the species. If this is so then it would add further weight to the argument that these two monomorphic races represent the primitive condition for the species (Trimen, 1869 and subsequently Poulton, 1924; Ford, 1936; Clarke \& Sheppard, 1963; Turner, 1963; O’Donald \& Barrett, 1973; Clarke et al., 1985).

The spectra from the lower surfaces of the wings (even in trimeni females, Figure 3-10e) show the same reflectivity (although tending to be a little less reflectant) over the Visible spectrum, but the UV peaks are very slightly higher, showing a slightly higher reflectivity to UV light than the upper surfaces (see Figure 3-11). This would make them appear a very slightly different colour (more shifted to the blue end of the spectrum) than the upper wing surfaces to an animal with colour vision which included UV.

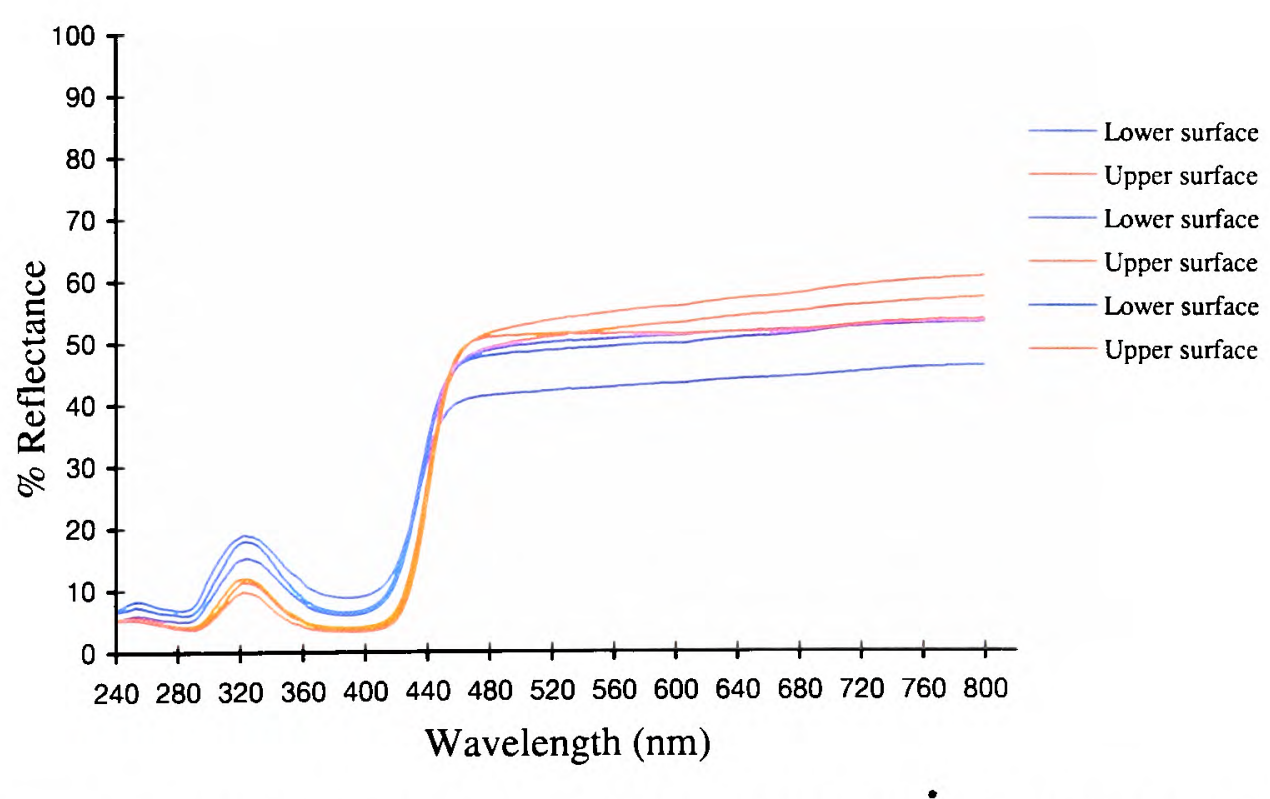

Figure 3-11 Comparison of the reflectance spectra from the upper and lower wing surfaces of males from race cenea

The spectra of Mylothris ngaziya (see Figure 3-10f) show that the pigment would easily be distinguished from that in Papilio dardanus by animals with UV vision. The reflectance rises slightly from about $40-50 \%$ at $800 \mathrm{~nm}$ until about $480 \mathrm{~nm}$, when it starts to decline, steeply from $440 \mathrm{~nm}$ until $400 \mathrm{~nm}$. From $400 \mathrm{~nm}$ downwards the reflectance is very low (under $10 \%$ ), markedly different from all the Papilio dardanus yellow spectra. This indicates that Papilio dardanus is very unlikely to be gaining protection from predators with colour vision including the UV through mimicry of Mylothris. 
a) Spectra of various orange colours

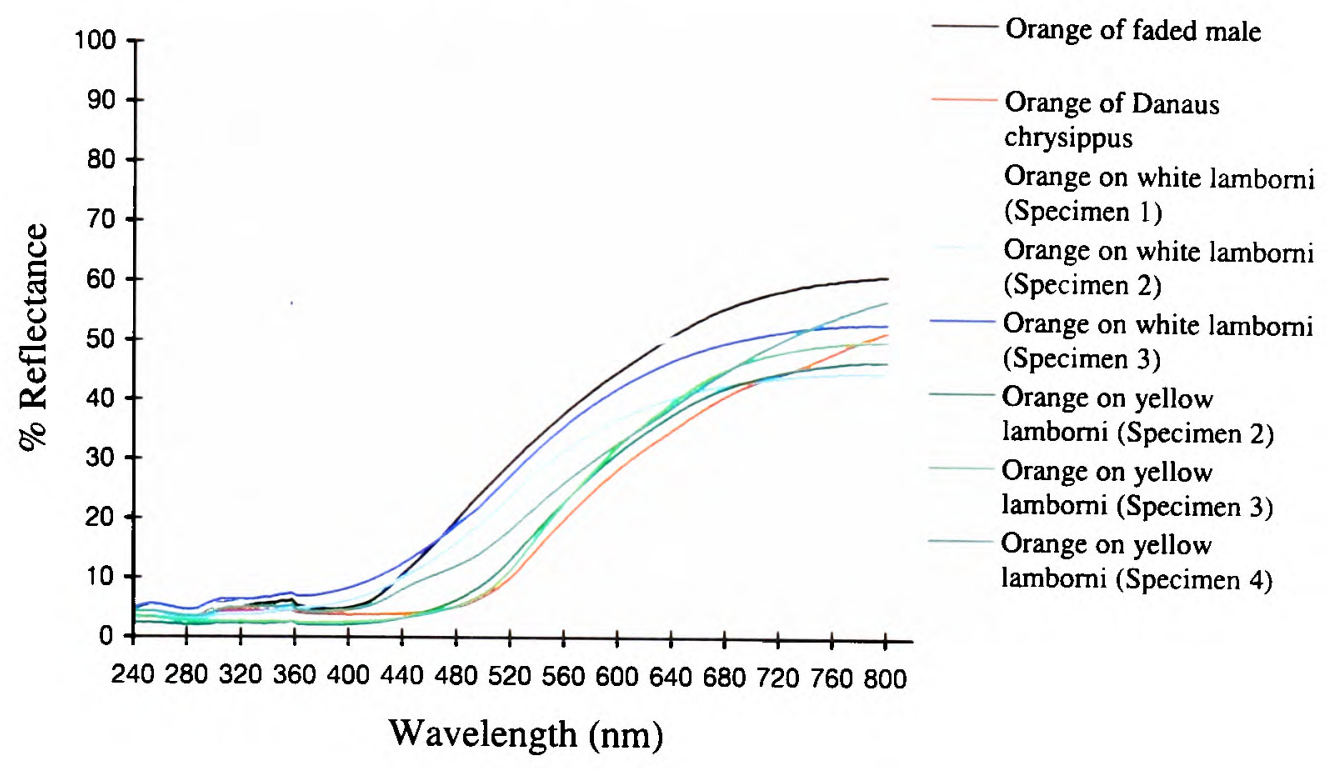

b) Comparison of faded and unfaded yellow

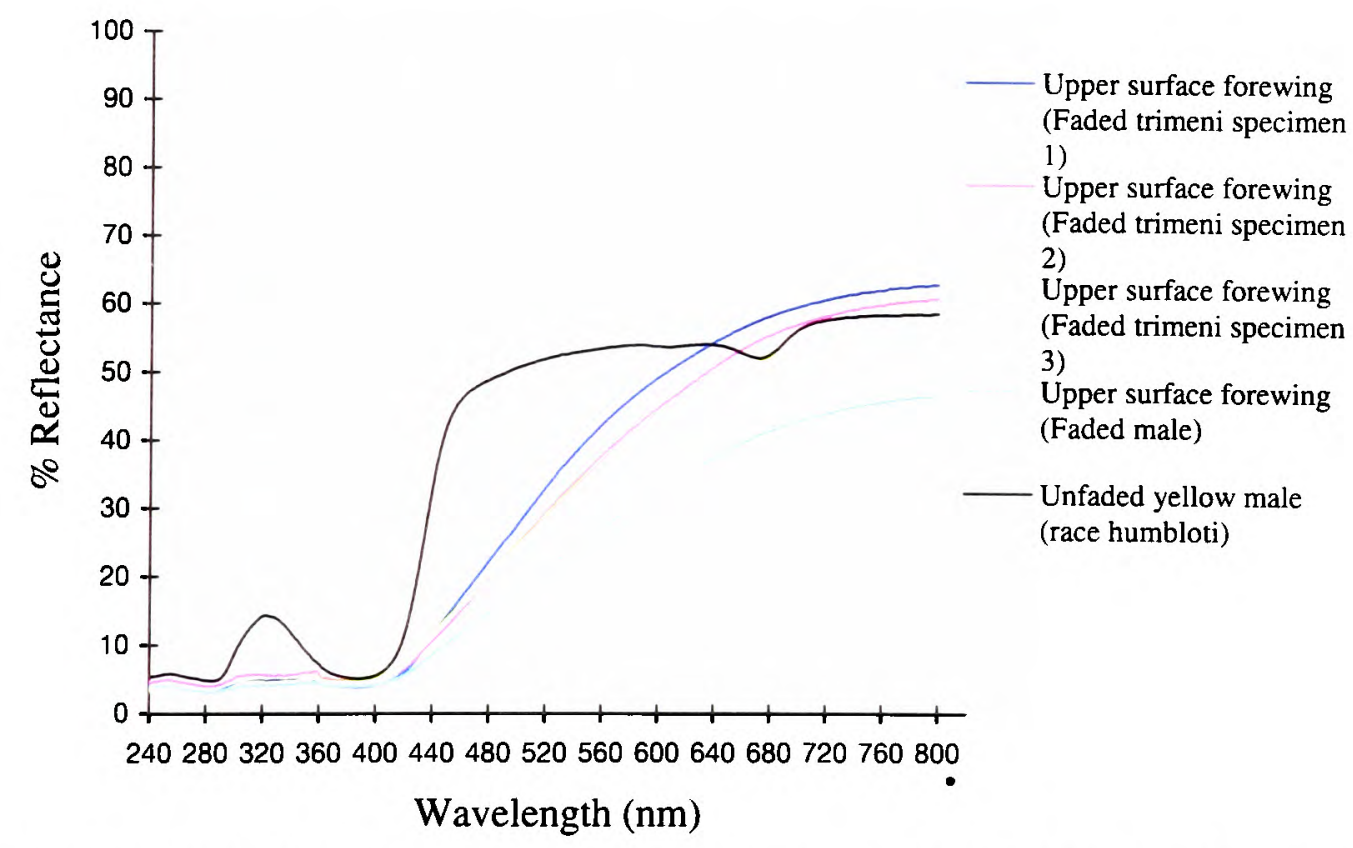

Figure 3-12 Reflectance spectra of a) the orange patches and b) a comparison of the faded yellow patches found in Papilio dardanus

The spectra of the faded yellow pigment show a dramatic change from those of the pigment when fresh (see Figure 3-12b). The reflectance at $800 \mathrm{~nm}$ is the same (around 50\%), but this slowly decreases to about $5 \%$ at $410 \mathrm{~nm}$, and remains this low throughout the UV range. Thus the reflectance is high through the red and quite high in the yellow, but is increasingly low through the green part of the spectrum, and lacks any reflectance in the blue and UV. This explains why we see a gradual reddening of the pigment, as it loses reflectancy of green (which, combined with red causes us to perceive yellow), and to a lesser degree reflectancy of yellow. As Figure 3-12b demonstrates, the faded yellow shows a dramatically different spectrum from that of the unfaded yellow (shown in 
black), losing the green/yellow and UV reflectancy which gives the unfaded yellow such a characteristic shape.

The spectra of the orange patches in yellow and white lamborni specimens from the tibullus race of Papilio dardanus are very similar to each other, and show essentially the same sort of smooth curve as in those of the faded yellow pigment (see Figure 3-12a). The curve of the orange in yellow

a) Spectra of the white patches from different races

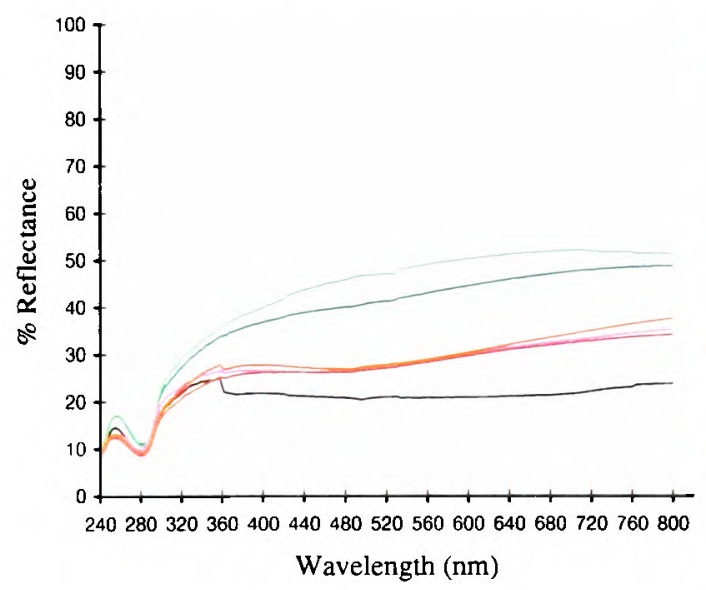

b) Spectra of white patches in hippocoonides from race tibullus

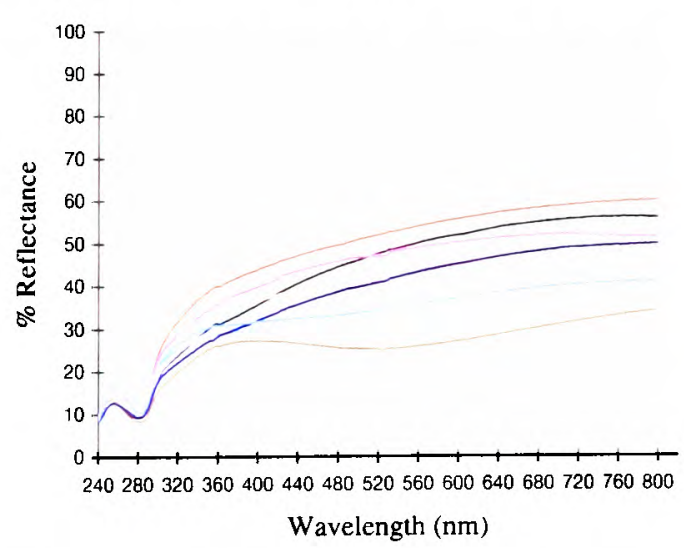

c) Spectra of the white patches in Amauris niavius

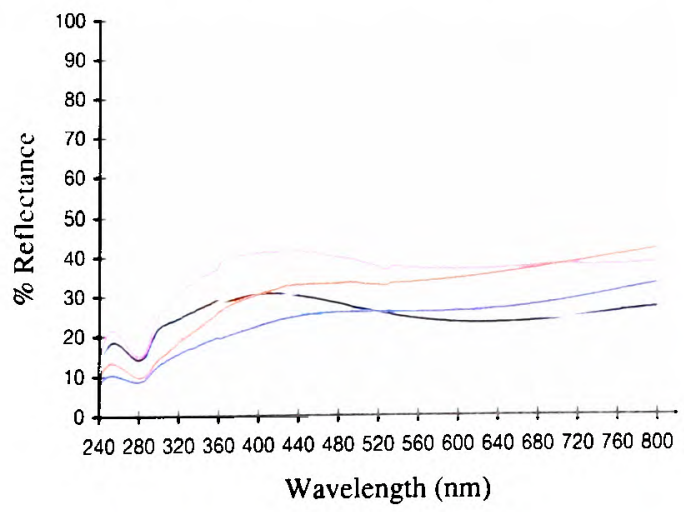

Figure 3-13 Reflectance spectra of the white patches found in a) Papilio dardanus specimens from different races, b) Papilio dardanus race tibullus hippocoonides specimens, and c) Amauris niavius. lamborni however (shown in green in Figure 3-12a), is a little more sigmoidal, with even less reflectance in the green part of the spectrum. This makes them a little redder in appearance to us. They all lack reflectance in the UV range (as was also demonstrated in Experiment 3$1)$.

The spectrum of the orange pigment in Danaus chrysippus is again a similar smooth curve (see Figure 3-12a) and is almost indistinguishable from the spectra of the orange patches in yellow lamborni.

The reflectance spectra of the white patches in the

- hippocoonides and white lamborni morphs of Papilio dardanus show a basically high level of reflection throughout the Visible spectrum, as would be expected, but the hindwings of hippocoonides have a lower reflectance over the Visible range (see Figure $3-13 \mathrm{~b}$, the hindwings are plotted in blues, and the forewings in reds/pinks). All individuals show a considerable drop 
in reflectivity between about $360 \mathrm{~nm}$ and $280 \mathrm{~nm}$, where the reflectivity is consistently $10 \%( \pm 2 \%)$. From here it again rises sharply to a small peak (between 10 and $20 \%$ reflectivity) at $250 \mathrm{~nm}$, and again drops off. Although the intensity of the reflection is variable, the shape of the curves in the UV region is extremely consistent. In the Visible spectrum there appear to be two possible patterns. Most specimens start with a high reflectivity at $800 \mathrm{~nm}(30-60 \%)$ which slowly decreases in a steady curve until around $360 \mathrm{~nm}$, where the drop-off becomes steeper. A few others start between $20-30 \%$ reflective at $800 \mathrm{~nm}$ and then decrease only very slightly, before either dropping off at $360 \mathrm{~nm}$, or rising from about $440 \mathrm{~nm}$ to $360 \mathrm{~nm}$ and then dropping. The specimens which tend to show this pattern are those from race cenea, or the white lambornis from race tibullus (see Figure 3-13a) although this difference could merely be due to the poor condition of these specimens (note in Figure 3-13b that the hindwing of specimen 2 , from race tibullus, also shows this pattern).

The lower surfaces of the wings show the same reflectance from $800 \mathrm{~nm}$ until $360 \mathrm{~nm}$, but from then on the reflectance tends to be slightly lower than that of the upper surfaces, although the reflectance follows exactly the same pattern (see Figure 3-13b where the lower surfaces are shown in dark colours). This difference is very slight, and probably falls within the range of individual variation.

The spectra obtained from specimens of Amauris niavius are very similar to those of the white patches in Papilio dardanus (see Figure 3-13c). They tend to follow the second pattern described above in the Visible region, with a slight decrease in reflectivity in the middle of the region and then a slight rise followed by a sharper drop in reflectance from $360 \mathrm{~nm}$ until $280 \mathrm{~nm}$. In the UV region the pattern of reflectivity is exactly the same. Again, there is no discernible difference between the upper and lower surfaces of the wings.

\section{Discussion}

The yellow pigment in males, trimeni females, and the yellow patches of yellow lamborni (prototrophonius) females all appear to be the same (Umebachi \& Yoshida's Papiliochrome II), although the slight difference in spectrum found from the island races humbloti and meriones may suggest that these two races are more closely related to each other than they are to the mainland races. The similarity of the yellow patches in all morphs which possess them means that little can be inferred from these results about the evolution of the various forms.

The spectra of the pale yellow wings of Mylothris ngaziya lacked the UV component found in the yellow patches of Papilio dardanus, indicating that to a predator with colour vision including UV the two species would not appear similar. 
Papiliochrome II was shown in Experiment 3-2 to fluoresce weakly in the Visible spectrum (between 420 and $630 \mathrm{~nm}$ ) under a wide range of UV and violet wavelengths (from $340 \mathrm{~nm}$ to $440 \mathrm{~nm}$ with a peak at about $400 \mathrm{~nm}$ ). Its reflectance spectrum shows that it is highly reflective to red, yellow and green light $(800-450 \mathrm{~nm})$, and the scales containing Papiliochrome 11 are also reflective to UV light with a wavelength around $320 \mathrm{~nm}$. The absorptive region from $360-420 \mathrm{~nm}$ is in accordance with the maximum absorption wavelength of the pigment, which is described by Umebachi and Yoshida (1970) to be at $380 \mathrm{~nm}$. This also matches the wavelengths which most excite fluorescence, seen in the excitation scan of the pigment with fluorescence at $483 \mathrm{~nm}$ in Figure 3-9, as demonstrated in Experiment 3-2. After prolonged exposure to sunlight, the yellow pigment loses its fluorescence and reflectancy to green light, appearing orange. In addition, the UV reflectancy of these scales is lost. The bases for both these phenomena are investigated further in Experiments 3-4, 3-5, and 3-7.

Experiment 3-2 demonstrated that there was no pigment present in the white patches of hippocoonides which fluoresced under UV light, but the patches are highly reflective of Visible and near UV wavelength light $(800 \mathrm{~nm}$ - nearly $300 \mathrm{~nm})$ and also reflective of UV light with a wavelength of about $255 \mathrm{~nm}$. The white patches in Amauris niavius show very similar properties. The spectra indicate that the hippocoonides morph of Papilio dardanus is indeed likely to be a convincing mimic of Amauris niavius although it is worth remembering that the integrating sphere collected light reflecting from all angles, and thus the two apparently similar spectra may have had very different angular components. Technically (Mason, 1926), true whites are all structural colours - an unpigmented cuticle will appear white due to the scattering of light by surface irregularities and air bubbles within the structure, and pigmental whites can be argued to be structural because they result from the scattering of light by colourless pigment granules. The small UV peak in both species is likely to be a structural colour caused by a more regular structural arrangement, and if this is so, then the fact that the peak in hippocoonides so closely matches that in Amauris niavius and yet is different from that in the yellow trimeni females suggests that hippocoonides has not evolved as a mimic simply through the loss of pigmentation in the yellow scales, but has actually changed the structure of the scales themselves to produce a sophisticated mimicry of the structural colours in Amauris niavius. Experiments 3-4 and 3-5 study the microstructure of the scales to investigate this further. It is interesting, however, that the small observed peak is so far into the UV range of the spectrum, as this is likely to be on the edge of the visual range of most animals, including the butterflies' presumed major predators, birds (see Chen \& Goldsmith, 1986). The fact that the hippocoonides spectrum so closely matches that of Amauris niavius in this region suggests that it is either under selection by predators in this region, or that this small peak is simply a consequence of similarities in the more general structure of the scales (e.g. that all unpigmented scales have a similar spectrum). This should also be clarified by microscopy in Experiments 3-4 and 3-5. 
The orange pigment found in the lamborni morphs does not fluoresce (as shown in Experiments 3-1 and 3-2), and shows a steady decrease in reflectancy across the Visible spectrum, and no reflectance of UV light. There is a very slight difference in the shape of the spectra from white and yellow lamborni females, with the latter showing a slightly sharper drop-off in reflectancy in the green region of the spectrum. The spectra from the orange in white lamborni specimens appears to be closer to that of the faded Papiliochrome II pigment, and that of the yellow lamborni specimens to be more similar to the properties of the orange pigment in Danaus chrysippus. It is thus very difficult to say from this evidence whether the lamborni morphs are convincing mimics of either.

The similarity between the white patches in hippocoonides and those on the forewings of the white lamborni morphs is intriguing. If indeed the hippocoonides morph has changed the structure of its scales in order to mimic Amauris niavius closely, then either the white lamborni morph has secondarily also changed the structure of its scales from that found in trimeni and yellow lamborni in order to mimic the white spots on the forewings of Danaus chrysippus, or it has evolved directly from the hippocoonides morph. It is unfortunate that the small size of the white spots in Danaus chrysippus made it impossible for their spectra to be analysed, as it is not known whether or not they share the same spectral shape as the whites in Papilio dardanus and Amauris niavius, and hence whether the white lamboni morph is indeed mimicking the colour of the spots. Again, a study of the microstructure of the scales should help clarify whether the similarity in the spectra of the whites is important or not.

In general, then, the white of hippocoonides and the white lamborni (and also of Amauris niavius) has been shown to be a 'true white' - reflectant to both light in our Visible spectrum and UV light. It is not yet known to what extent Papilio dardanus can detect light as far into the UV region as $200-300 \mathrm{~nm}$, so it is not yet known whether the reflectancy in this area will affect their perception of the colour. This is investigated in Experiment 3-6.

Most vegetation does not reflect UV wavelengths, and is dominated by wavelengths greater than 450nm (Klein, 1978; Menzel, 1979), and hence appears to us as it would to animals with a visual spectrum including both the Visible spectrum and extending into the UV. The spectral sensitivities of Papilio dardanus are investigated in Experiment 3-6 to determine how the morphs might appear. However, the white morph will be distinct from the background by nature of its 'brightness'. Although it has been argued (Kevan et al., 1996) that insects cannot detect white against a vegetation background due to the fact that they cannot detect differences in brightness, this argument is negated by experiments which clearly show that butterflies can (Swihart, 1971; Scherer and Kolb, 1987).

However, the yellow trimeni females might not be so obvious, as their UV reflectance would cause their overall perceived colour to an animal with UV sensitivity to be pulled down slightly towards the blue end of the spectrum, probably into the green region. Their brightness may also be comparable to 
that of the background. It is possible, therefore, that the intense yellow that we perceive is actually far less obvious against a green vegetation background to animals with UV sensitivity than might be expected.

\section{Experiment 3-4 Scanning Electron Microscopy of butterfly scales}

\section{Introduction}

Butterfly scales have been studied extensively using electron microscopy, and their general structure is well known (see Figure 3-2, and also Ghiradella 1991 for a summary). The ridges described in the introduction to this chapter are separated by horizontal crossribs in most species, but in Papilionids this is replaced by a network of fine tubes. Ghiradella has described six mechanisms by which structural colours may be formed, all of which are illustrated by Papilionid species (Ghiradella, 1985, and again see Figure 3-2), but most of these rely on the general method of structural colour formation of thin-film interference. The optical effect of four of the six methods (methods A-D in Figure 3-2) can be approximated as a multiple thin-film interference filter (Land, 1972), in which light is reflected off each surface in turn through a stack of layers with differing optical thickness, and interference between the reflected wavelengths gives rise to an iridescent structural colour (see Figure 3-14 and Land, 1972 for greater detail). Thus spectral components arising from structural colours may be predicted using this model (eg. Anderson \& Richards, 1942; Lippert \& Gentil, 1959; Ghiradella et al., 1972) when the number, thickness and spacing of the layer-forming structures is known.

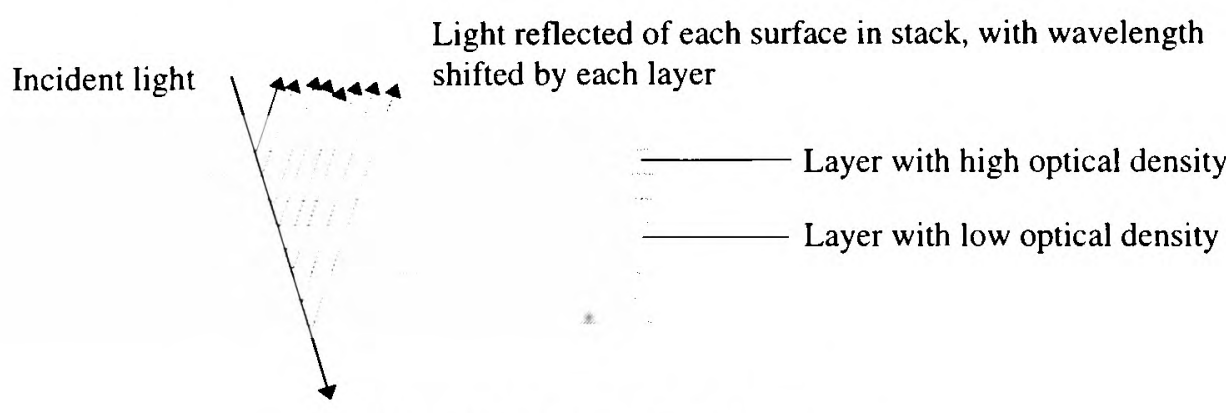

Light transmitted through the layers

Figure 3-14 The theory of multiple thin-film interference (after Land, 1972)

In Experiments 3-1 and 3-3, UV reflectance was demonstrated from the white and yellow scales of Papilio dardanus, and also from the white scales of Amauris niavius. By investigating the structure of the scales using Scanning Electron Microscopy (SEM) it should be possible to compare the microstructures of the scales of different colours, and also compare scales of Papilio dardanus with those of Amauris niavius and Danaus chrysippus, two of its putative models (Trimen, 1868). It is hoped that this study of the microstructure of the scales will confirm that the UV reflectance is 
indeed due to structural reflectance. SEMs have been obtained at a high enough resolution to measure the widths and spacings of lamellae (for example see Bingham et al., 1995), and so if thin-film interference is the mechanism producing structural colours in any of the scales studied, it may be possible to predict these using a mathematical model of this mechanism, and compare these predictions with the measured UV reflectance from the scales to confirm that this reflectance has a structural origin.

If the UV reflectance does indeed have a structural origin, it is hoped that the microstructure of the scales might elucidate whether the hippocoonides morph has truly evolved to mimic the UV reflectance of Amauris niavius so closely (see Experiment 3-3) or whether this is merely a coincidental similarity arising from general similarities in the structure of the scale, and to compare the methods of reflectance in the two species. It is also hoped that the structure might give a clue as to how the sunlight-faded individuals have come to lose their UV reflectance so dramatically, as was also shown in Experiment 3-3.

\section{Aim}

The aim of this experiment is to determine whether or not the UV reflectance seen in white and yellow scales of Papilio dardanus is likely to have a structural origin, and if so to determine the mechanism by which the colours are produced, and how the reflectance may be lost with exposure to sunlight.

\section{Method and Materials}

Small portions of wing were mounted flat in the electroconductive substance DAG on SEM stubs and sputter coated with gold. They were viewed in a Phillips 515 SEM. Individual scales were also sliced in half perpendicular to the longitudinal ridges under a microscope using a razor blade. These were then mounted on end, with the cut edge uppermost. The fine structure of the scales was photographed on monochrome Kodak T-Max 3200 film.

The following scales were mounted:

- White scales from Amauris niavius.

- White scales from the hippocoonides morph of Papilio dardanus.

- Orange scales from a lamborni morph of Papilio dardanus.

- $\quad$ Orange scales from Danaus chrysippus.

- $\quad$ Faded yellow/orange scales from an old male Papilio dardanus.

- Yellow scales from a male Papilio dardanus race cenea (upper and lower wing surfaces).

- Yellow scales from a male Papilio dardanus race tibullus. 
- Yellow scales from a male Papilio dardanus race humbloti.

- Black scales from a male Papilio dardanus.

\section{Results}

1) Coarse structure of the scales, as visible under magnification of approximately $\times 200$ to $\times 500$ :

Figure 3-15 clearly shows the two types of scales present in Papilio dardanus - cover scales and ground scales. The cover scales are long and thin with two rounded lobes at the tip. These are interspersed amongst the slightly shorter and wider ground scales, which have three rounded lobes at the tip. Figure 3-16 demonstrates the same cover and ground scales in Danaus chrysippus. The cover scales are again slightly longer than the ground scales, although not as long as the scales in Papilio dardanus, and are simply rounded at the tips (there are no lobes present). The ground scales are also shorter than those of Papilio dardanus, and have four lobes at the tip (occasionally the central two lobes merge into one). In the top right hand corner, some scales have been displaced, and their sockets on the upper wing membrane are visible.

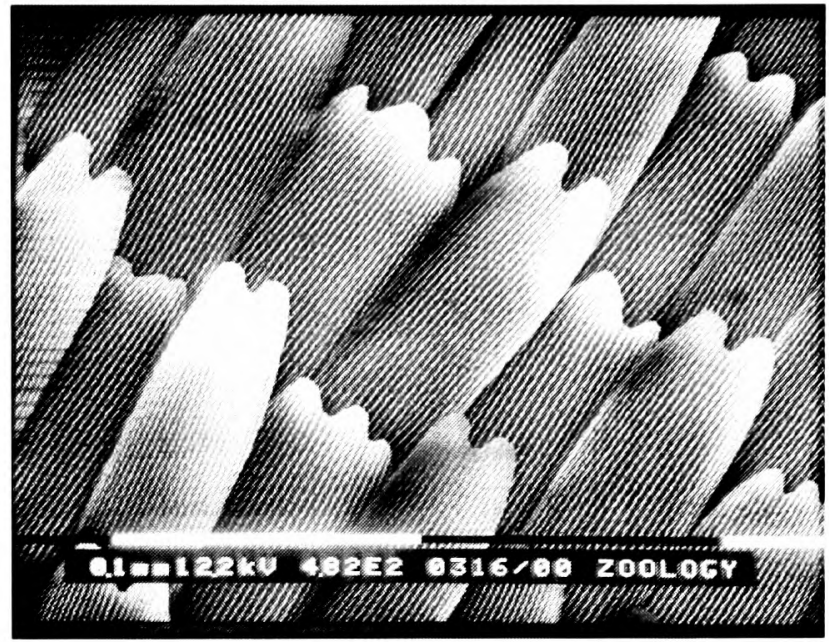

Figure 3-15 Low power SEM of scales on wing of Papilio dardanus. (orange scales from lamborni morph) showing two-lobed cover and three-lobed ground scales. (black \& white bars $=100 \mu \mathrm{m}$ )

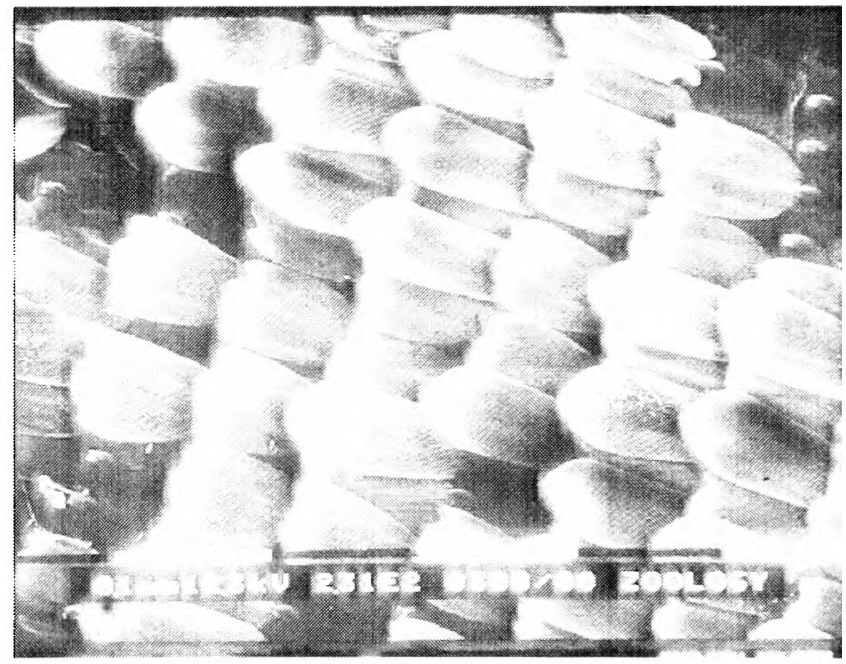

Figure 3-16 Low power SEM of Danaus chrysippus scales showing unlobed cover scales and four-lobed ground scales. (black \& white bars $=100 \mu \mathrm{m}$ ) 
2) Fine structure, as visible with a magnification of $x 3500$ :

a)

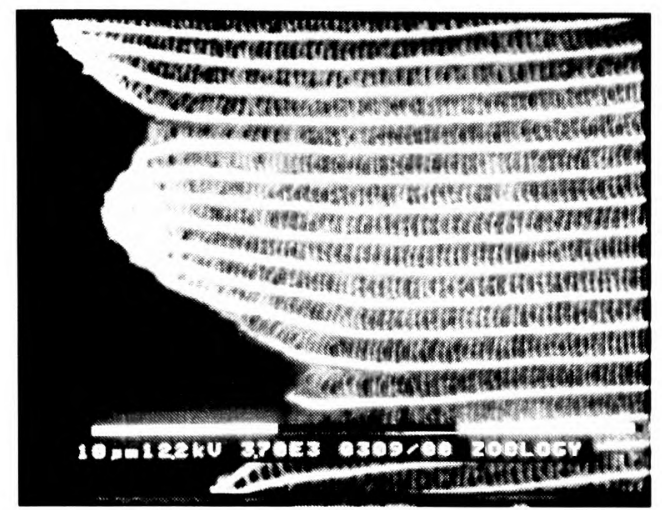

b)

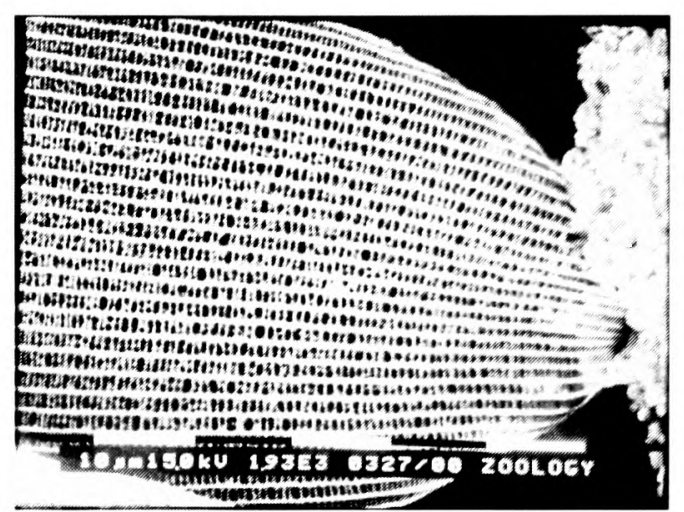

Figure 3-17 High power SEM of scales from a) Danaus chrysippus, and b) Amauris niavius (black \& white bars $=10 \mu \mathrm{m}$ )

The longitudinal ridges on the scales of Danaus chrysippus and Amauris niavius can be seen to be joined by simple crossribs (see Figure 3-17). In Danaus chrysippus the ridges (measured using Manostat calipers accurate to $0.05 \mathrm{~mm}$ ) are approximately $1.59 \mu \mathrm{m}$ apart (all exactly the same within the accuracy of the measuring technique). In Amauris niavius the spacing between the ridges is approximately $2.00 \mu \mathrm{m}$ (again all exactly the same).

a)

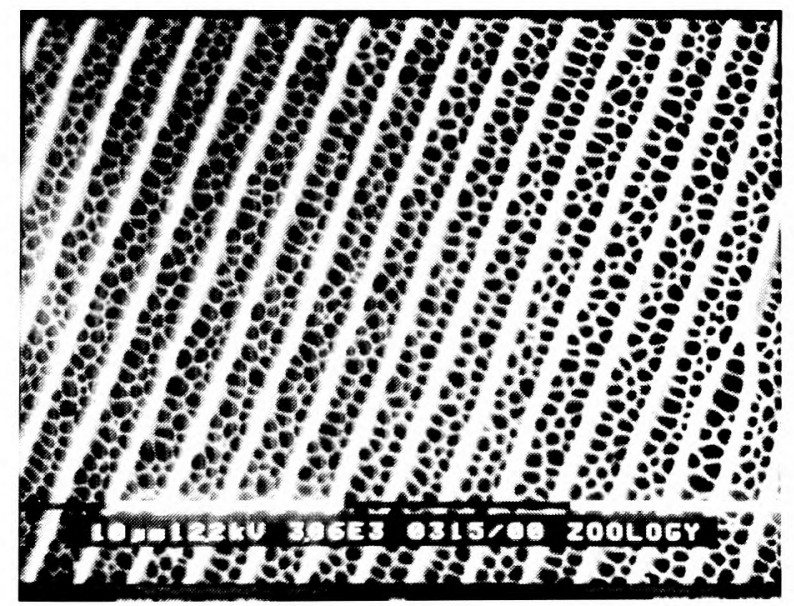

b)

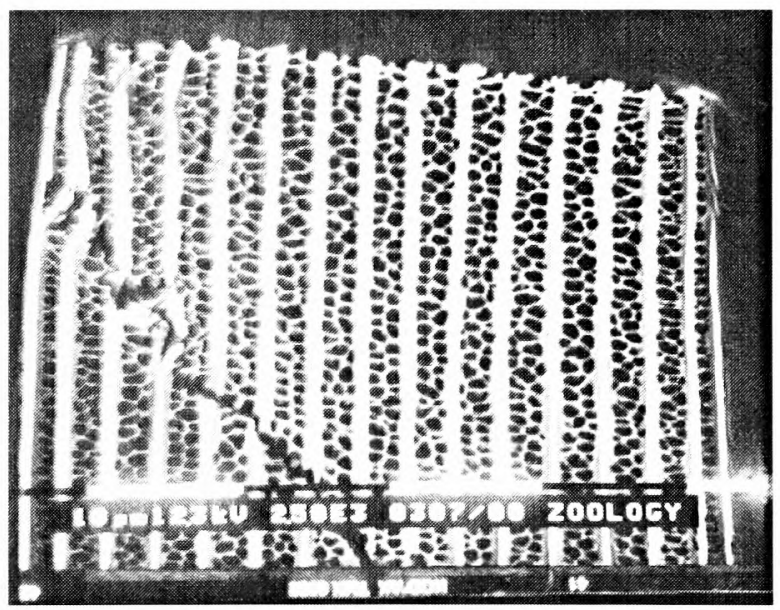

Figure 3-18 High power SEM of scales from Papillio dardanus: a) orange scale from lamborni female (magnification $\times 3860$ ), b) white scale from hippocoonides female (black \& white bars $=10 \mu \mathrm{m}$ ).

The ridges on the scales of Papilio dardanus can be seen to be joined by a net-like reticulum (see Figure 3-18), as would be expected in a Papilionid butterfly (Ghiradella, 1985). In the orange scales the ridges are all approximately $2.09 \mu \mathrm{m}$ apart, and the diameter of the holes in the net is relatively large. In the white scales the ridges are all about $3.12 \mu \mathrm{m}$ apart, and the holes are similarly sized. 
a)

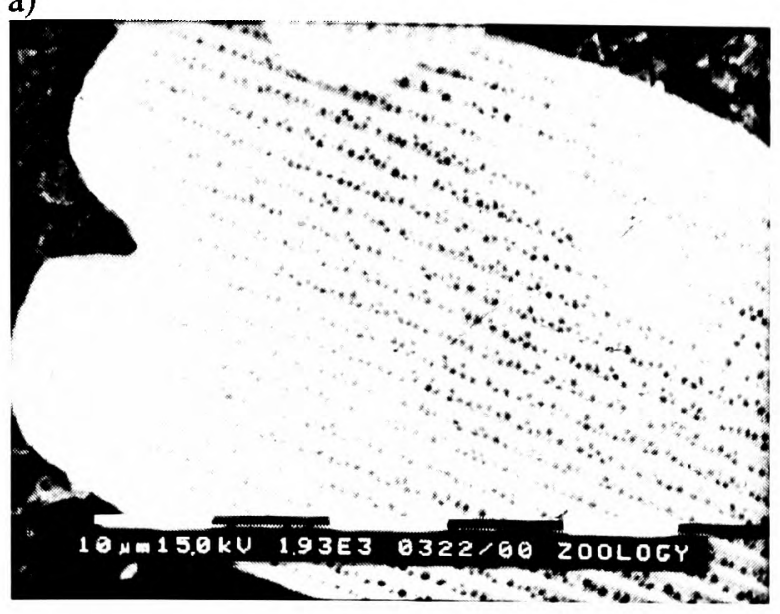

c)

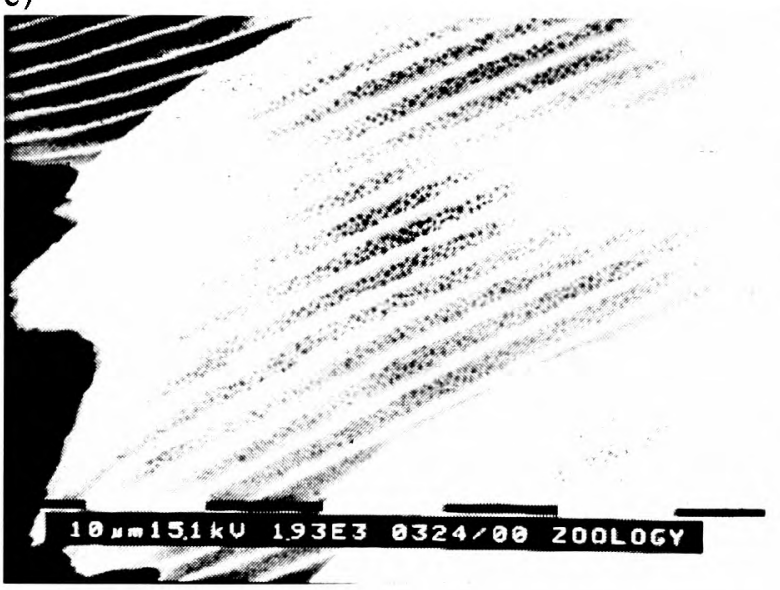

b)

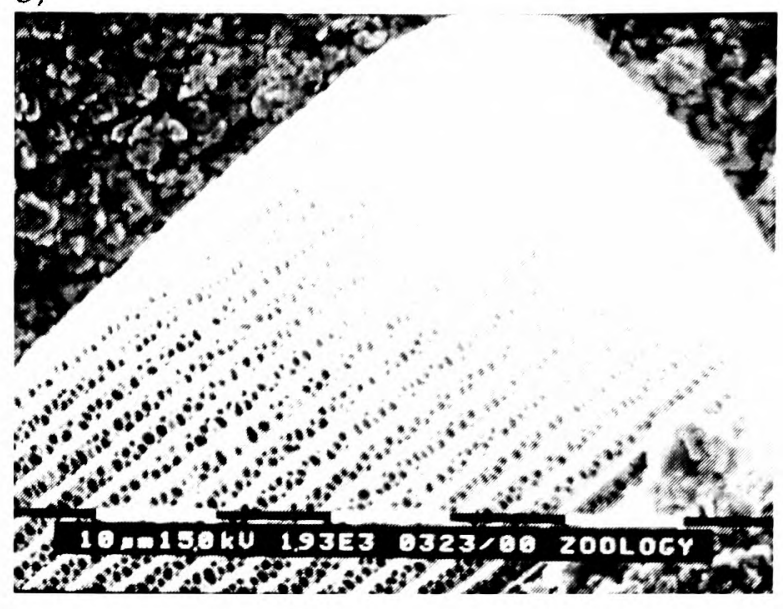

d)

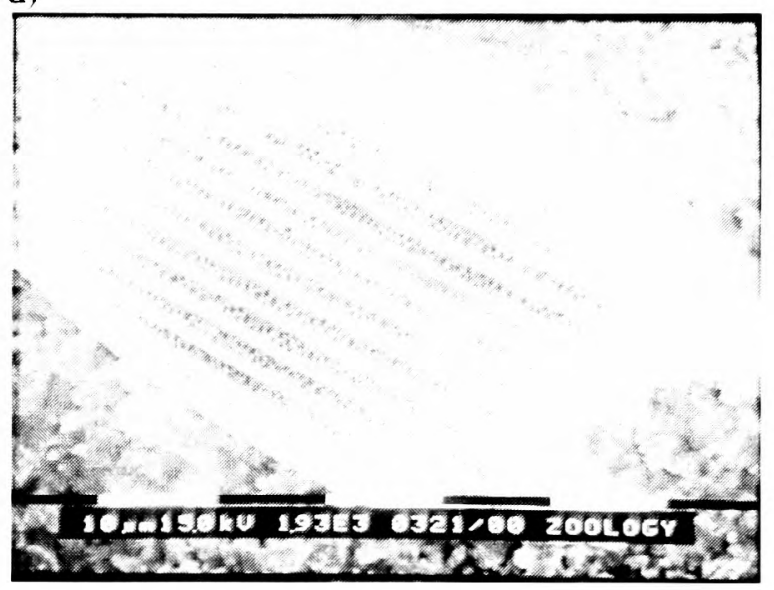

Figure 3-19 High power SEM of scales from Papilio dardanus: a) yellow scale from male Papilio dardanus (race humbloti), b) yellow scale from the underside of the wing of male Papilio dardanus (race cenea), c) faded yellow/orange scale from old male Papilio dardanus d) black scale from male Papilio dardanus (black \& white bars $=10 \mu \mathrm{m}$ )

In yellow scales from any race the ridges all have a spacing of about $2.73 \mu \mathrm{m}$, on both upper and lower wing surfaces (see Figure 3-19). The holes in the net appear to be smaller than in the other coloured scales. There was no obvious difference between the yellow scales from young Papilio dardanus individuals and the faded yellow scale from an old individual. In the black scales the ridges are approximately $2.46 \mu \mathrm{m}$ apart, and the net holes appear to be similar to those on the yellow scales.

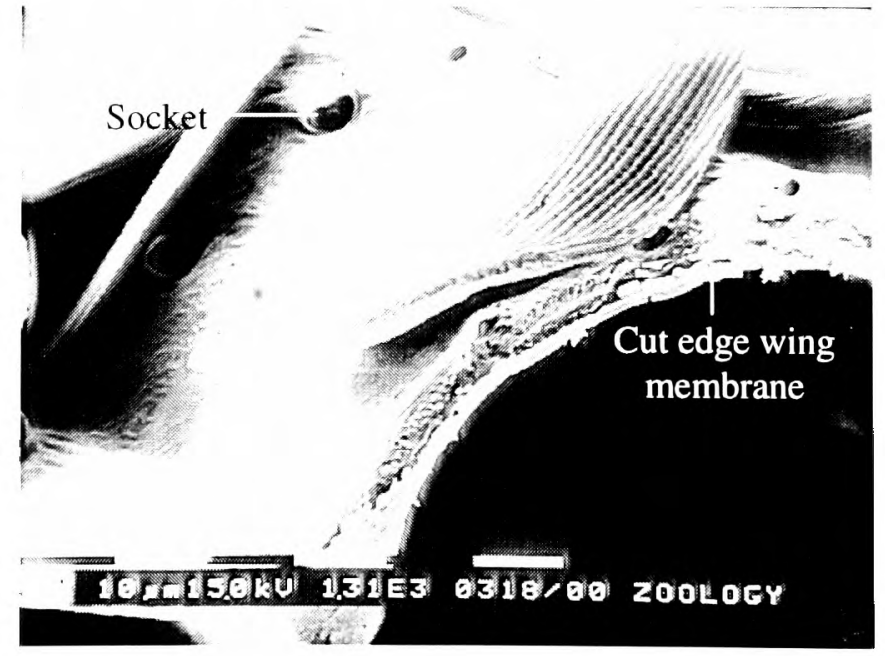

Figure 3-20 High power SEM of a scale from Papilio dardanus cut in half, still mounted on the wing membrane, but displaced from its socket (visible at the top of the picture, to the left). Magnification x 1310 . 
The mounting of scales with their cut ends visible proved to be very difficult, and of those that were successfully mounted, no focused image could be obtained of the cut end, owing to the vibration of such a thin sample in the beam of electrons (see Figure 3-20).

\section{Discussion}

Although the SEM technique did not allow a focused image of the lamellae to be obtained, the general scale structures of Papilio dardanus, Amauris niavius and Danaus chrysippus could be compared. Papilio dardanus, as expected, showed a different scale structure from the other two species, illustrating the typical Papilionid reticulum form (Ghiradella, 1985). Differences between scales of different colours were also noted, although it is very unlikely that these differences will have any effect on the structural colours, as the variable distances - between the ridges, and the diameter of the holes in the reticulum - are far larger than the wavelength of light in the near UV and Visible regions of the spectrum (200-700nm), and thus will not cause any interference effects.

Therefore, to answer the questions posed in the introduction to this experiment, Transmission Electron Microscopy, in which the sample is fixed in resin and then sliced very thinly, is necessary as this prevents the sample from vibrating in the electron beam.

\section{Experiment 3-5 Transmission Electron Microscopy of butterfly scales}

\section{Introduction}

Transmission Electron Microscopy (TEM) allows ultrathin slices of material, fixed in resin, to be viewed at very high magnification. The technique has already been used successfully to investigate butterfly scale structure (e.g. Ghiradella et al., 1972). It can give a very clear image of a slice through the scale, and will reveal whether or not structures are present which would lead to the production of structural colours. This study should therefore reveal whether or not the UV reflectance measured in Experiment 3-3 can be attributed to structural reflectance or not.

In addition, if the structural colours are formed by the method of thin-film interference, as outlined in the introduction to Experiment 3-4, then it should be possible to measure the widths and spacings of the structures forming the layers from the TEM images, and thus use a mathematical model of thin-film interference (see introduction to Experiment 3-4) to predict whether or not the structure seen is consistent with the reflectance measured in Experiment 3-3 (as done by Ghiradella et al., 1972). However, the slices are very difficult to prepare and cut, and if measurements are to be taken of the layer-forming structures, care needs to be taken that the slices are exactly perpendicular to 
the basal membrane of the scale (a diagonal cut will give artificially enlarge measurements, importantly those of the width and spacings of the layer-forming structures).

TEM images of the yellow/orange scales of an old individual of Papilio dardanus may also suggest how the loss of UV reflectance seen in Experiment 3-3 arises because if the reflectance is structural in origin then its disappearance is likely to be due to a degradation of the reflecting structures.

\section{Aim}

The aim of this experiment is to determine the method of structural colour formation in Papilio dardanus, using a mathematical model, if possible, to test whether or not the structure observed is likely to result in the colours produced.

\section{Method and Materials}

Small sections of wing were cut and placed in $1 \%$ osmium to stain for 1 hour. They were then put through a series of alcohol concentrations $(70 \%, 80 \%, 95 \%$ and absolute), being in each for 30 minutes, to desiccate them. After that they were placed in 'absolute dry alcohol' (absolute alcohol over sodium sulphate), followed by a 50:50 solution of absolute alcohol and epoxypropane (propylene oxyde) and then one hour in pure epoxypropane. The sections were left overnight in a 50:50 mixture of araldite and epoxypropane with a lid on. The next day the mixture was allowed to vaporise before the sections were embedded in araldite. The araldite blocks were left in an oven overnight at $60^{\circ} \mathrm{C}$, and then left to cool. Ultrathin slices were taken with a diamond Reichert-Jung Ultracut onto 400 bar grids. The slices were left to stain in saturated uranyl acetate for 10 minutes and then lead citrate for 5 minutes, and then viewed using a Philips EM 400 transmission electron microscope.

The following scales were examined:

- White scales from a hippocoonides female of Papilio dardanus.

- White scales from Amauris niavius.

- Yellow scales from a male Papilio dardanus.

- $\quad$ Orange scales from a faded male Papilio dardanus.

- Orange scales from a yellow lamborni female of Papilio dardanus.

- Black scales from a male Papilio dardanus.

\section{Results}

It was interesting to see that the ridges both Papilio dardanus and Amauris niavius had the same reflective structure, despite the differences in the gross structure of the scales as seen under the SEM in Experiment 3-4. Photographs were taken under a magnification of 28,000 and measurements 
of the lamellae and air spaces taken off prints enlarged 5 times. A piece of software called Multilayer (Version 1.1, produced by Randy Geels ${ }^{*}$ ) was then used to model thin-film interference (see Land, 1972 for a mathematical explanation of the process). The accuracy of the software was tested by predicting the structural colours in Eurema lisa and Morpho rhetenor using data from papers by Ghiradella et al. (1972) and Bingham et al. (1995) respectively, and found to be reasonable.

A cross section of a white

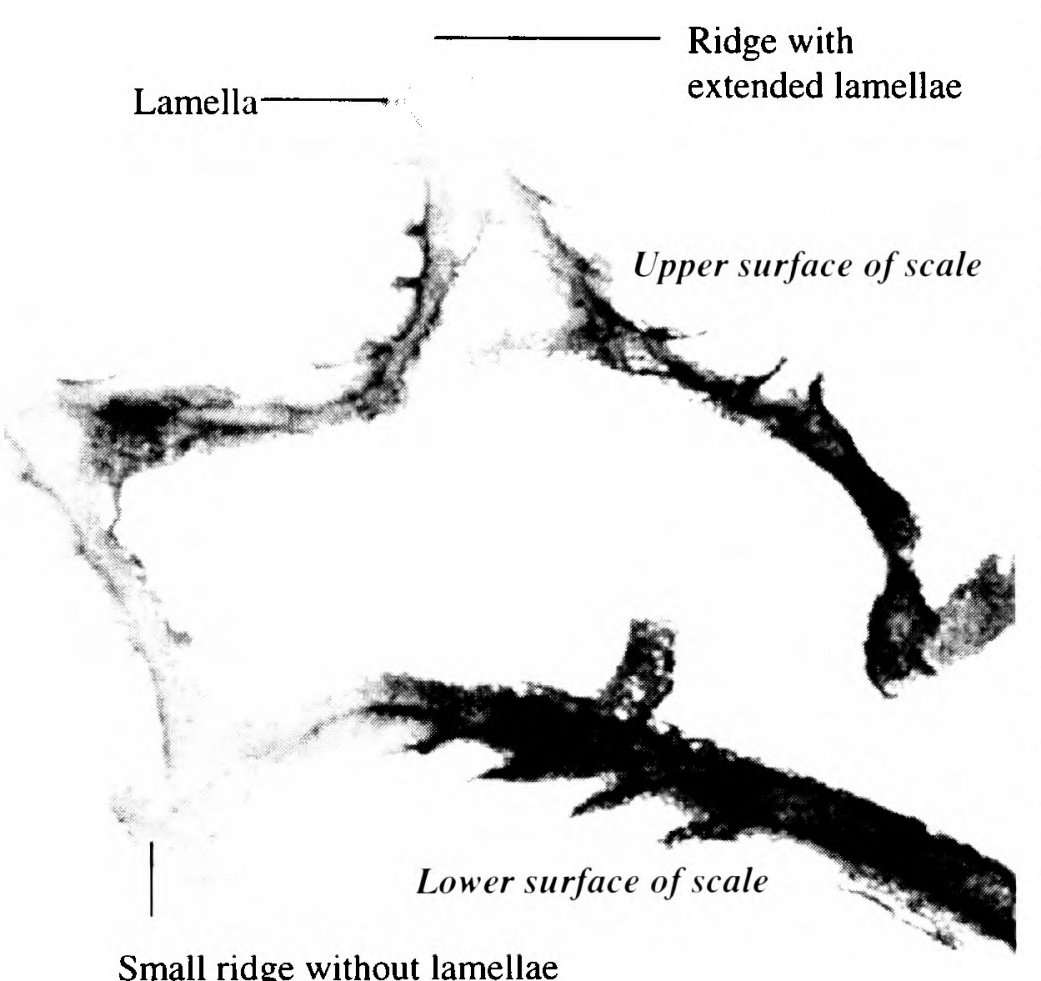

Small ridge without lamellae

Figure 3-21 TEM of the cross-section of the end of a white hippocoonides scale

Papilio dardanus hippocoonides scale clearly shows the tall ridges exhibiting the classic 'Christmas Tree' structure of ridges causing lamellar thin-film interference (see Figure 3-2) on the upper surface in contrast to the small, mushroom-shaped ridges carrying no structural reflectors on the lower surface (see Figure 3-21). The inside of the scale appears completely hollow, indicating that the colour of these scales is entirely due to reflectance, and that no pigment is present. Measurements of the widths of the lamellae and air spaces were taken off one side of one ridge which was best in focus. There are, on average, 5 complete lamellae on each side of the ridges (average taken from counting the lamellae on 6 sides), each with an mean width of $0.0297 \mu \mathrm{m} \pm 0.0052 \mu \mathrm{m}(\mathrm{n}=5)$ and with an average air space between them of $0.0821 \mu \mathrm{m} \pm$ $0.0097 \mu \mathrm{m}(\mathrm{n}=4)$. These values give a predicted major reflectance peak of $252 \mathrm{~nm}$, using Multilayer.
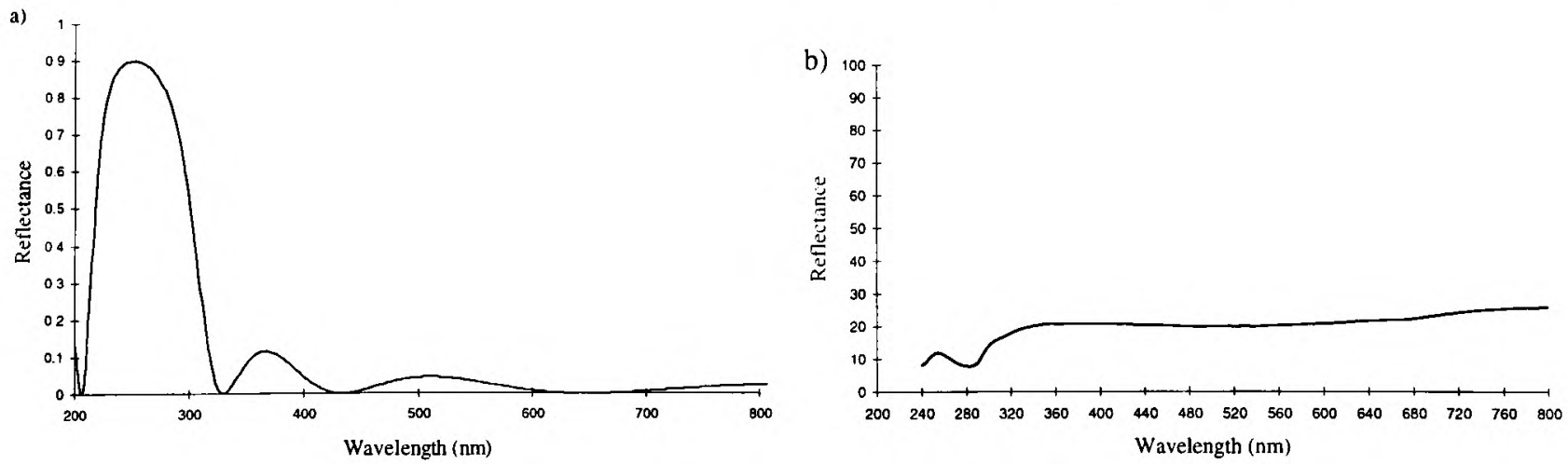

Figure 3-22 a) Multilayer prediction (number lamellae $=5$, width $=0.030 \mu \mathrm{m}$, air spaces $=0.082 \mu \mathrm{m}$ ) compared with b) experimentally obtained spectra of white patches in hippocoonides

" e-mail: info@filmetrics.com 
Figure 3-22a shows the Multilayer prediction, with the very large primary peak indicating the structural colour predicted (the smaller peaks show negligible reflectance at other wavelengths). Figure 3-22b shows a typical spectrum obtained in Experiment 3-3 for the white patches in hippocoonides, illustrating the small UV peak at $255 \mathrm{~nm}$ (the rest of the spectrum being due to pigmental reflection). The Multilayer prediction from the measurements of the lamellae matches this almost exactly, suggesting that this small peak visible in the reflectance spectrum is indeed caused by the lamellae acting as a thin-film interference filter.

The cross sections of white scales from Amauris niavius (see Figure 3-23) show essentially the same structure as those of Papilio dardanus, with tall ridges and a series of exaggerated lamellae. It is interesting to note, however, that the interior of the scale is not hollow, as seen in hippocoonides (Figure 3-21). It is therefore possible that there is some pigmentation in the white scales of Amauris niavius. There are about 5 complete lamellae on each side of the ridges (average taken from counting the lamellae on seven complete sides of ridges), with an mean width of $0.039 \mu \mathrm{m} \pm 0.006 \mu \mathrm{m}(\mathrm{n}=33)$. The air spaces are an average of $0.083 \mu \mathrm{m} \pm 0.017 \mu \mathrm{m}$ wide $(\mathrm{n}=28)$. Multilayer predicts a maximum reflectance of $286 \mathrm{~nm}$ using these figures, which is slightly higher than that obtained experimentally (around $255 \mathrm{~nm}$ ). This difference would be caused by a slight overestimate of the width of the lamellae in the model. In the case of Amauris niavius the lamellae were very difficult to measure as the photographs taken were slightly out of focus in all cases. The resulting blur around the edges of the lamellae therefore probably caused the lamellae to be recorded as slightly wider than they actually are. However, it is still close to that obtained for hippocoonides (see Figure 3-24 and Figure 3-22) and it seems very likely that in both cases the measured UV reflectance peak is caused by the lamellae on the ridges acting as thin-film interference filters.

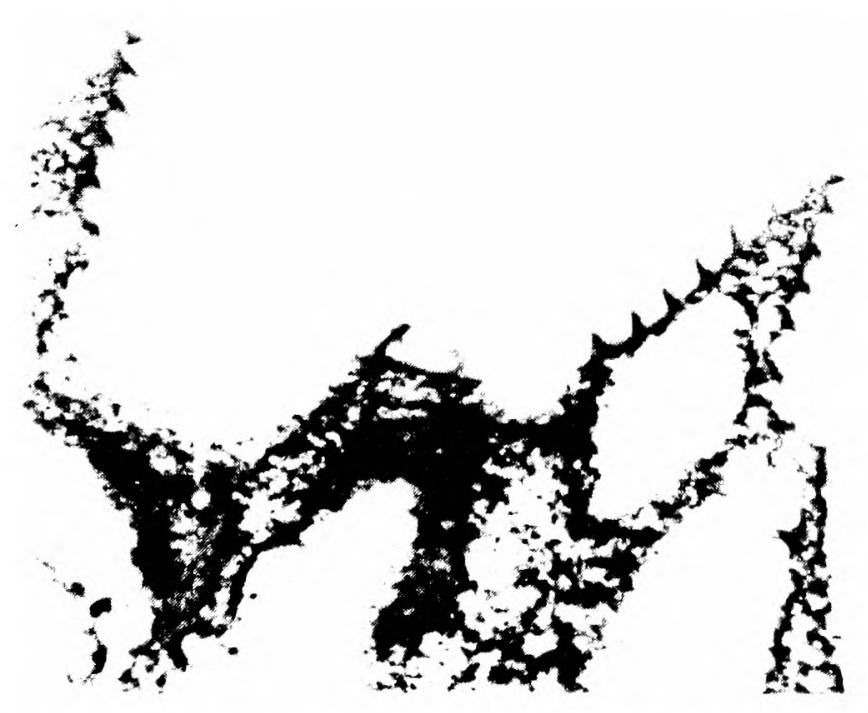

Figure 3-23 TEM of the cross section through two ridges on a scale of Amauris niavius 

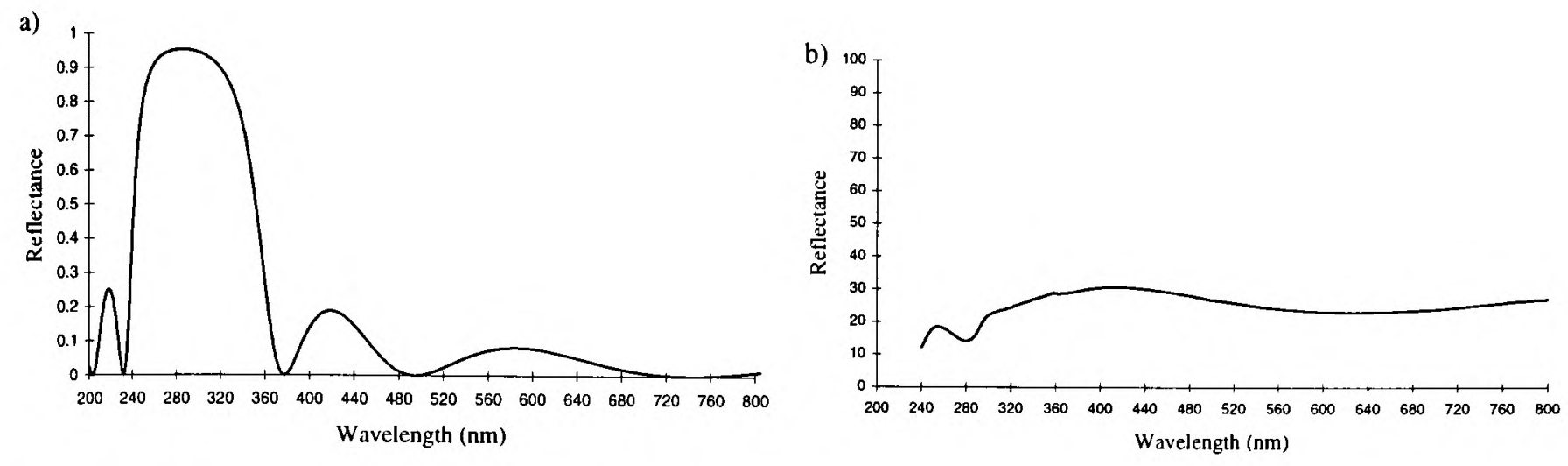

Figure 3-24 a) Multilayer prediction (number lamellae $=5$, width $=0.039 \mu \mathrm{m}$, air spaces $=0.083 \mu \mathrm{m}$ ) compared with b) experimentally obtained spectrum of white patches in Amauris niavius.

Figure 3-25 TEM of the cross sections through the edge of a yellow scale from male Papilio dardanus.

accurate average measurement of the widths, an average was taken of the measurements of all the lamellae and air spaces from two complete sides. Although the sample size is therefore small, measuring the lamellae from only the top or bottom of the other ridges would bias the result one way or the other. The lamellae have an average width of $0.042 \mu \mathrm{m} \pm 0.015 \mu \mathrm{m}(\mathrm{n}=13)$ and the air spaces between them an average width of $0.084 \mu \mathrm{m} \pm$

The yellow scales from a male Papilio dardanus (see Figure 3-25) are very similar to the white scales, with about 6 complete lamellae on each side of the ridges. Since the lamellae and the spaces between them increase down the ridge, for an

a)

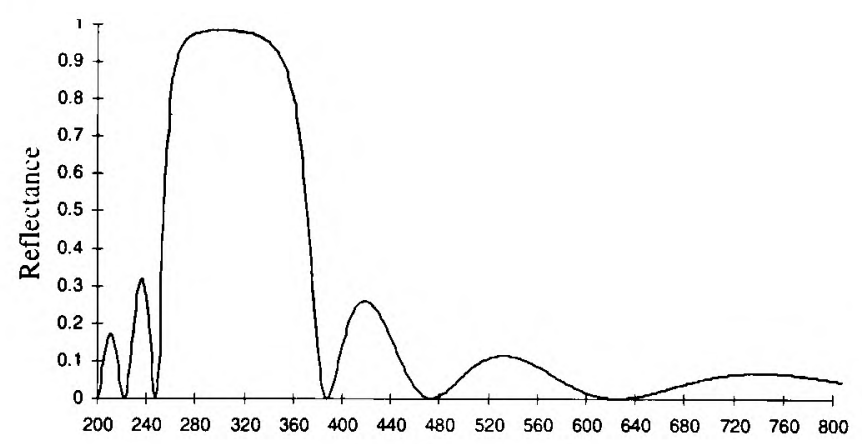
Wavelength (nm)

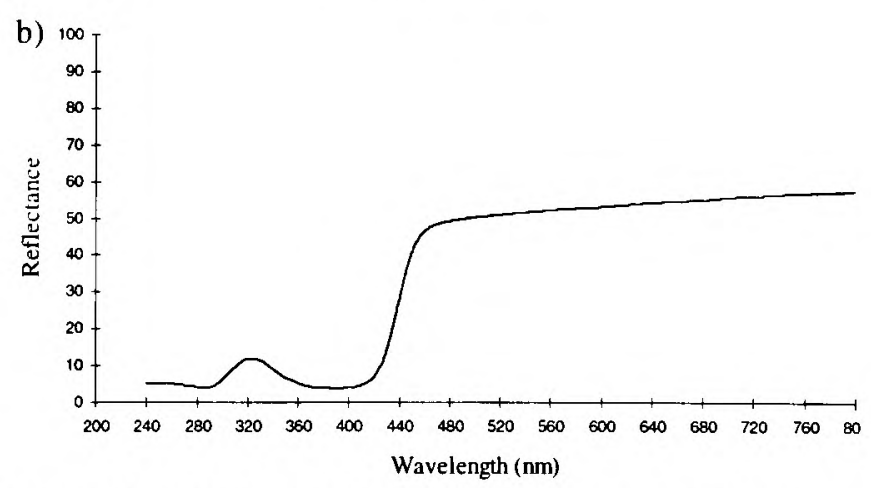

Figure 3-26 a) Multilayer prediction (number lamellae $=6$, width $=$ $0.042 \mu \mathrm{m}$, air spaces $=0.084 \mu \mathrm{m}$ ) compared with b) experimentally obtained spectrum for yellow patches in Papilio dardanus. 
$0.018 \mu \mathrm{m}(\mathrm{n}=12)$. Using Multilayer, this gives a prediction of a maximum reflectance around $300 \mathrm{~nm}$, which is close to that measured experimentally, around $322 \mathrm{~nm}$ (see Figure 3-26). The interior of the scale is also not hollow as in hippocoonides (see Figure 3-21), but instead filled with what are presumably pigment granules containing Papiliochrome II. Again it is possible to see small ridges on the underside of the scale without structural reflectors. This difference between the yellow scales and white scales of hippocoonides means that the scales in hippocoonides have been structurally altered in order to produce the UV reflectance, which matches that found in Amauris niavius.

One scale from a faded yellow male Papilio dardanus shows possible damage to the lamellae on one side (see Figure 3-27). The lamellae are bent or broken on one side of the ridge, and this is seen on most of the ridges on the scale viewed. Although the evidence from only one scale is weak, this damage to the lamellae may be the explanation for the loss of UV reflectance in the faded specimens.

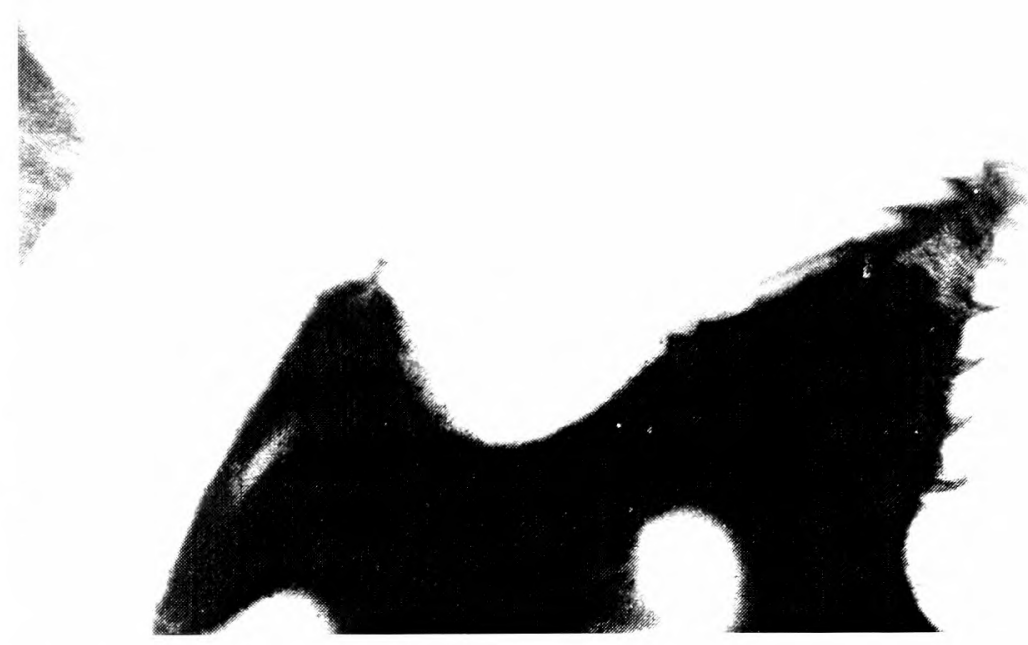

Figure 3-27 Cross section through a ridge on a faded yellow scale

The orange scales of the yellow lamborni morph of Papilio dardanus have ridges which are markedly different from those in the white and yellow scales (see Figure 3-28 compared with Figure 3-21 and Figure 3-25). They are much shorter, and more widely spaced, with only an average of 3 lamellae, and these lamellae are much thicker - averaging $0.114 \mu \mathrm{m} \pm 0.039 \mu \mathrm{m}$ wide $(n=10)$, with air spaces of $0.133 \mu \mathrm{m}$ $\pm 0.030 \mu \mathrm{m}(\mathrm{n}=7)$. These measurements produce a Multilayer prediction of no UV

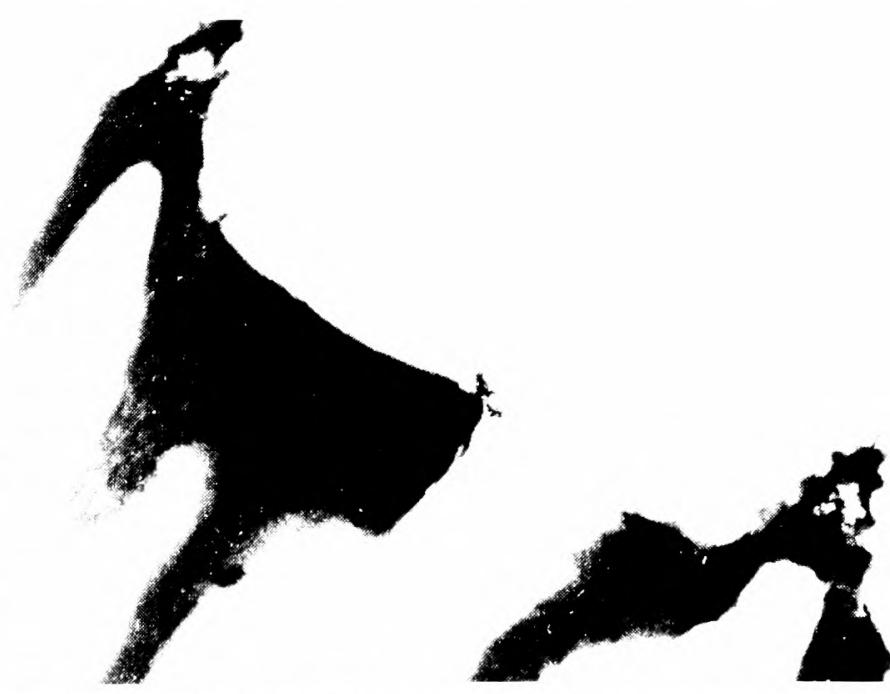

Figure 3-28 TEM of cross section through a ridge on an orange scale from Papilio dardanus. reflectance (see Figure 3-29) - the predicted peak in the visible spectrum is relatively low and shallow, rather than being sharply defined and with a high intensity as seen in the other predictions, and so is unlikely to cause any measurable reflection even in this region of the spectrum. 

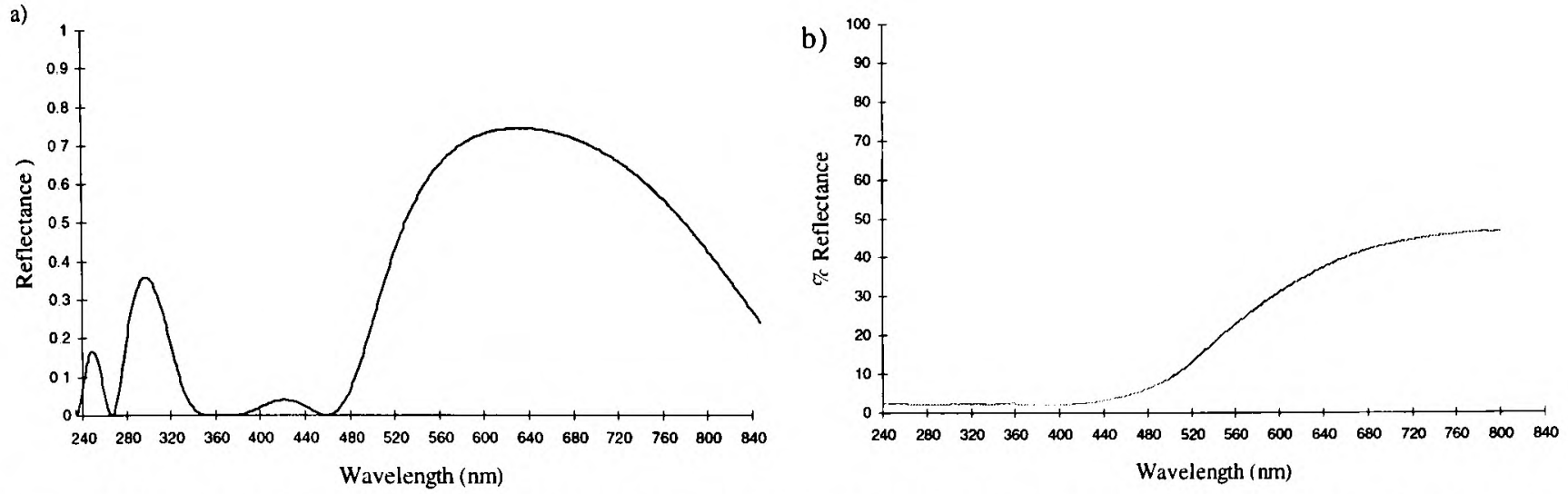

Figure 3-29 a) Multilayer prediction (number lamellae $=3$, width $=0.114 \mu \mathrm{m}$, air spaces $=0.133 \mu \mathrm{m}$ ) compared with b) experimentally obtained spectrum for orange patches in Papilio dardanus.

The black scales (taken from a young male Papilio dardanus) (see Ridge (with one lamella)

Figure 3-28) show a great difference in the structure of the ridges from the other scales investigated. The ridges are identical on both the upper and lower surfaces of the scale. They are all squat and mushroom-shaped with only the occasional protruding lamella. This means that they cannot act as thin-film stack reflectors. The interior of the scale is hollow, as melanin is usually distributed

Lower surface of scale

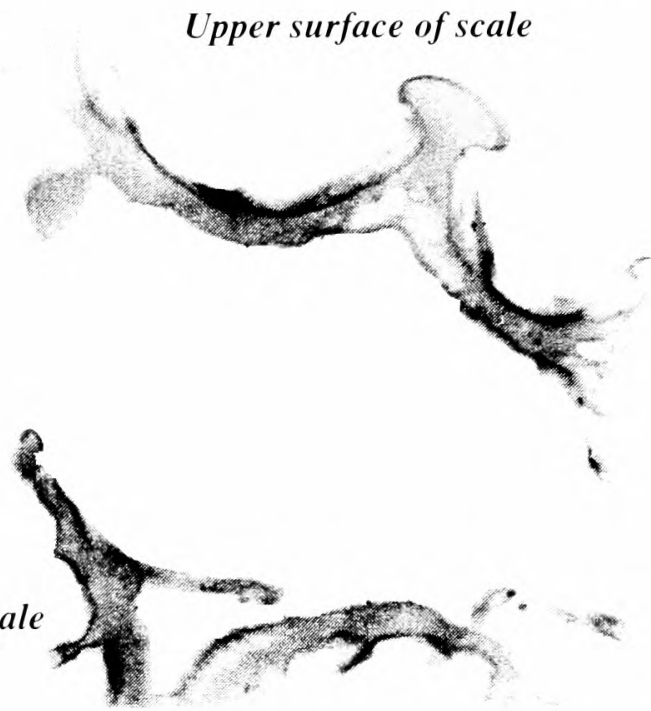
throughout the structure, rather than being sequestered into granules

Figure 3-30 TEM of the cross section through a black scale from Papilio dardanus (Ghiradella, 1984).

\section{Discussion}

The microstructure of the scales gives an interesting insight into the evolution of the species and its mimicry. The measurements of the number and widths of the lamellae allow a good prediction of the structural colours of the scales to be made when included in a thin-film interference model of reflectance (slight differences between the predicted and measured wavelengths are almost certainly due to the difficulties of accurate measurement of the lamellae and air spaces). Therefore it can be concluded that it is indeed these lamellae which cause the peaks seen in the UV region of the reflectance spectra. The yellow scales in Papilio dardanus have a different UV peak (322nm) from the white scales in the female hippocoonides morph $(255 \mathrm{~nm})$, whilst the UV peak in hippocoonides 
exactly matches that measured in its model, Amauris niavius, and this can be seen to be due to a slight narrowing of the widths of the lamellae on the scale ridges.

There is a lively debate as to how the different morphs evolved, and from what primitive condition. Trimen's original (1868) suggestion that the species was originally monomorphic, with both males and females showing the pattern now illustrated by males (as is the case in the monomorphic island races humbloti and meriones) has been adopted by one camp (including Poulton, 1924; Ford, 1936; Clarke \& Sheppard, 1963; Turner, 1963; O’Donald \& Barrett, 1973; Clarke et al., 1985). Another group (including van Bemmelen, 1922; Bernardi, 1963; Vane-Wright \& Smith, 1991), however, have not been happy with this view as the male pattern appears to be so far derived from the traditional swallowtail pattern, whilst the mimetic females illustrate many more of the primitive swallowtail elements (see Nijhout, 1991 for further details on the patterning). Recent mitochondrial DNA analyses comparing Papilio dardanus races tibullus, meriones, humbloti, and also Papilio phorcas, Papilio constantinus (thought to be its two sister species) and Papilio nobilis (a Papilio species with a superficially similar appearance to the males, not thought to be closely related)(VaneWright et al., in press) appear to indicate that humbloti is indeed the most primitive race, although it is stressed that this result is only tentative, and further analyses, including more races, are ongoing. However, if the yellow patterning is indeed ancestral to the hippocoonides morph, then the white coloration has evolved not simply through the loss of Papiliochrome II in the yellow areas, but also by an adjustment of the size of the lamellae to produce an exact mimicry of the reflectance spectrum of its model Amauris niavius. The fact that the white in white lamborni has exactly the same reflectance spectrum of those in hippocoonides also appears either to have involved the same alteration of the scale structure from the yellow scales found in yellow lamborni (if the white lamborni morph is derived from yellow lamborni), or, as appears more likely from this evidence, the white lamborni morph evolved directly from hippocoonides, inheriting the scale structure of the white patches. If the latter scenario is correct then the evolution of the yellow lamborni morph requires an explanation.

It is interesting that the orange scales have lamellae, bit these do not produce any reflection. In this way they are very different from the black scales. It is possible that they have evolved relatively recently from yellow scales (or white scales), and have not yet lost their lamellae completely. Whether they will or not depends on the cost of producing these lamellae which do not reflect.

The faded yellow scale shows the possibility that lamellae become damaged when exposed to sunlight, thus causing the loss in UV reflectancy seen in Experiment 3-3. If this is so, then we would also expect the white scales to lose their UV reflectance. This has yet to be studied. However, a larger number of scales of each colour need to be investigated to allow any firm conclusions to be drawn. The accuracy of measurements would also be greatly improved if an electron microscope with 
a digital display were used. This would allow accurate measurements to be taken directly from the sample. The difficulty of preparation precluded a greater range of individuals and scales being investigated in this study.

\section{Experiment 3-6 Electroretinogram of Papilio dardanus}

\section{Introduction}

The previous experiments in this chapter have provided an objective assessment of what colours are actually present on the wings of Papilio dardanus (and its models). However, it is now necessary to measure what colour information the butterflies actually receive, before behavioural colour choice experiments can be interpreted.

Electroretinograms (ERGs) have been used to ascertain the visual sensitivities of many lepidoptera (see Experiment 2-4). The usual way to represent these is by plotting the spectral efficiency function - a graph of the electrical response of the retina to different wavelengths of light at constant intensity (quantum flux). By ascertaining the visual range of Papilio dardanus, and to which colours the butterfly is particularly sensitive, it should be possible to assess how the different morphs might appear to the butterflies themselves. This may also may help to explain any preferences for particular morphs the males might show (investigated in Chapter 5), as some morphs may appear brighter and more obvious to them, or preferences shown for particular flower colours (studied in Chapter 4). As discussed in Experiment 2-4, ERGs are usually carried out using a 500W xenon lamp, and using filters to bring the quantum flux for each wavelength down to the level of the lowest intensity wavelength. However, as pointed out by Menzel $(1979, \mathrm{p} 507)$, the intensity function at each wavelength may be very different (i.e. the neuronal response could be non-linear with respect to intensity and different at each wavelength), and therefore the efficiency spectra usually plotted are only meaningful for the intensity at which they are recorded. In order to produce a more useful 'response surface' to assess the butterflies visual sensitivities a range of light intensities, the response of butterflies would have to be measured at different intensities for each wavelength.

\section{Aim}

The aim of this experiment is to measure, by use of an ERG, the visual sensitivities of Papilio dardanus to different wavelengths and intensities of light, and to use this, together with the reflectance spectra of the morphs obtained previously, to assess the relative colours of the different morphs as seen by other individuals. The information will also be used in Chapter 4 , which is a study of the colour preferences of Papilio dardanus with respect to flowers. 


\section{Method}

The wings were removed from an adult specimen of Papilio dardanus, and the insect was inserted into a tight plastic tube to prevent any movement of the limbs or antennae. Only the eyes protruded from the end of the tube. Two thin silver wire electrodes were then inserted just below the surface of one eye - one near the dorsal surface of the eye and the other nearer the ventral surface. The tube containing the butterfly was placed within a metal Faraday cage to shield the electrodes from electrical noise. A single beam of light could be allowed to enter the cage from a $200 \mathrm{~W}$ mercury lamp. The light was passed through a lens containing continuously flowing water to remove the far Infra Red wavelengths and prevent overheating. The light was then passed through a Spectral Energy GM252 monochromator and finally focused onto the butterfly's eye with a silicon lens. The potentials from the recording electrode were passed through a Isleworth preamplifier (amplified x 1000 with no frequency cuts) and to both a Tektronix Type 502A dual-beam oscilloscope and a Lloyd Instruments PL3 chart recorder.

The insect was dark-adapted for at least 5 minutes, and was then given brief flashes of monochromated light at $25 \mathrm{~nm}$ intervals $(+/-2 \mathrm{~nm})$ between 200 and $850 \mathrm{~nm}$. 'Runs' of flashes between these wavelengths were repeated 5 or 6 times for each individual, and the procedure was repeated for 8 individuals in total ( 5 males and 3 females). Since the wavelength responses of the butterfly are due to a complex interaction between the response of the light-sensitive pigments and the fine structure of the eye (Menzel 1979, p518), and are therefore not predictable, it was important to repeat the procedure on a number of individuals and take an average result to obtain as accurate a result as possible.

This procedure gave the responses of the retina to light of the highest intensity which could be produced by the mercury lamp (much higher than a corresponding xenon lamp). However, the intensity of a mercury lamp is by no means constant across the spectrum, so this was measured using a photodiode, RS stock number 305-462 (RS Electronics ') (see Appendix 2 for further details). A set of filters (numerical swatch set, Lee Filters**) was then calibrated using the photodiode at the peak wavelengths of the lamp (375nm, 450nm, 550nm), as discussed in Experiment 2-4. The response of four individual butterflies was thus recorded in the same way to differing intensities of these three wavelengths. Since these showed that there was little or no variation in the intensity functions of different individuals, and that the data formed smooth curves with little noise, the surface was constructed using multiple readings from only one individual. This had the advantage that the butterfly was not moved throughout the series of readings, and so the same proportion of the beam was falling on the eye at all times from the lamp. If different individuals had been used, inevitable

\footnotetext{
"RS Electronics, 34443 Schoolcraft, Livonia, Michigan 48150, USA

${ }^{* *}$ Lee Filters: Central Way, Walworth Industrial Estate, Andover, Hampshire SP10 5AN
} 
variations in the way they were mounted in the beam would have caused differences in the illumination of the eye by the beam and hence of the received intensities, and the readings from different individuals would have been shifted up or down the intensity curve slightly relative to one another by unknown amounts (see Appendix 2 for full details).

\section{Results}

The wave-form of a lepidopteran ERG is described as a monophasic negative response (Eguchi et al. 1982). This is seen as a characteristic 'spike' above background noise in response to light stimulation:

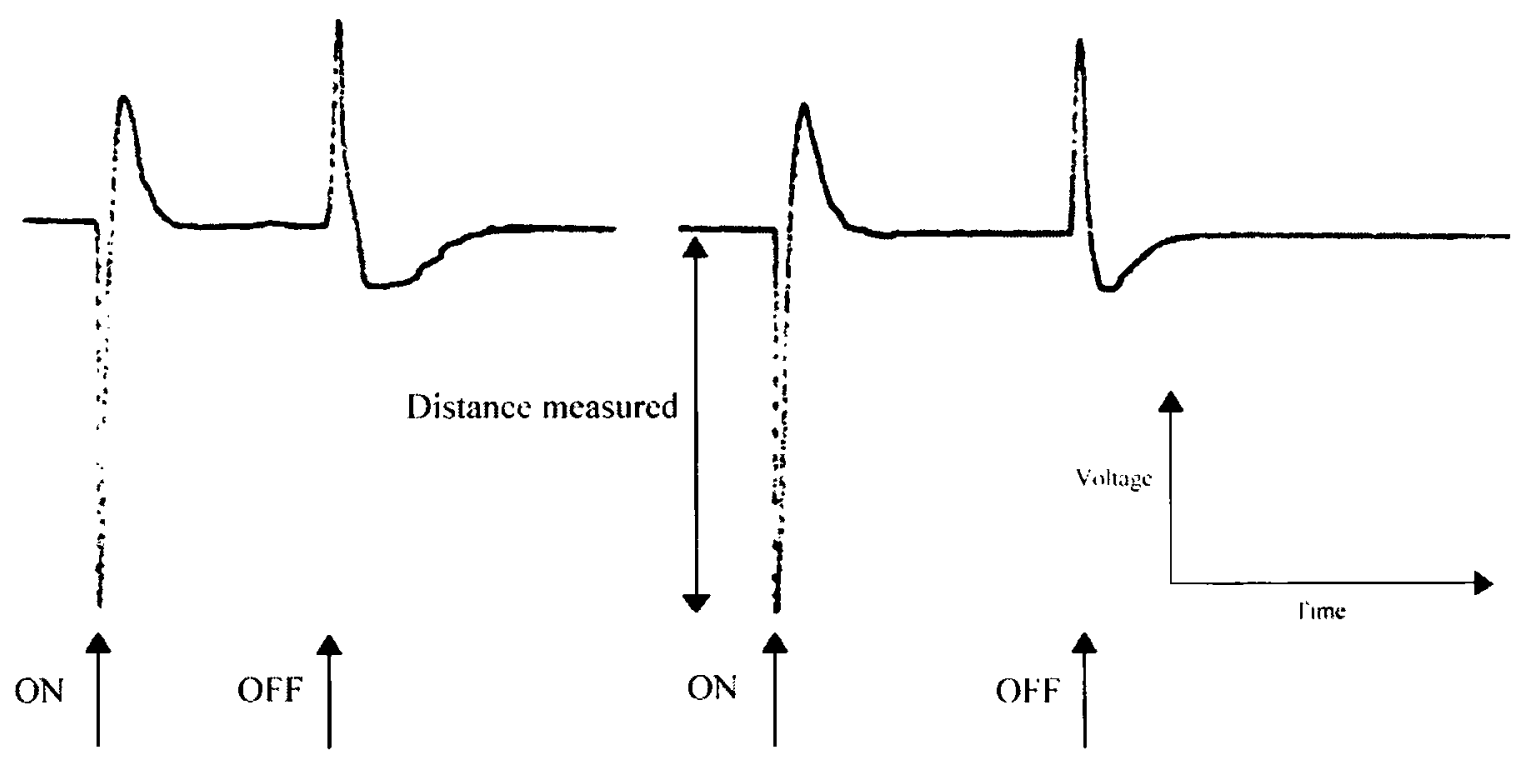

Figure 3-31 The recorded spike of electrical activity from the retina in response to a flash of light.

Occasionally an initial positive response is seen, which is a response from the optic ganglion layers (Swihart \& Gordon 1971, Eguchi et al. 1982), but this was ignored. The mean response of each individual insect to each wavelength was calculated (over the number of runs carried out).

All the individuals, both males and females, showed a very similar spectrum of sensitivity to wavelength, but on varying scales of amplitude due to the differing placements of the electrodes and of the illuminating beam relative to the eye. The individual spectra were therefore normalised with respect to each other. This was done by selecting one spectrum (the one with the largest amplitude), and calculating the ratio of each point in an individual spectrum to the respective point in the selected spectrum. The mean ratio between each spectrum and the selected spectrum could then be calculated. Each point in each individual spectrum was then multiplied by this mean ratio for the particular spectrum to scale all the spectra with respect to that selected. Then a mean spectrum for all individuals was calculated. To avoid biasing the result due to the initial selection of one spectrum to 
which to normalise all the others, this mean spectrum was then used as the 'selected spectrum' and the process iterated until a true mean spectrum was achieved.

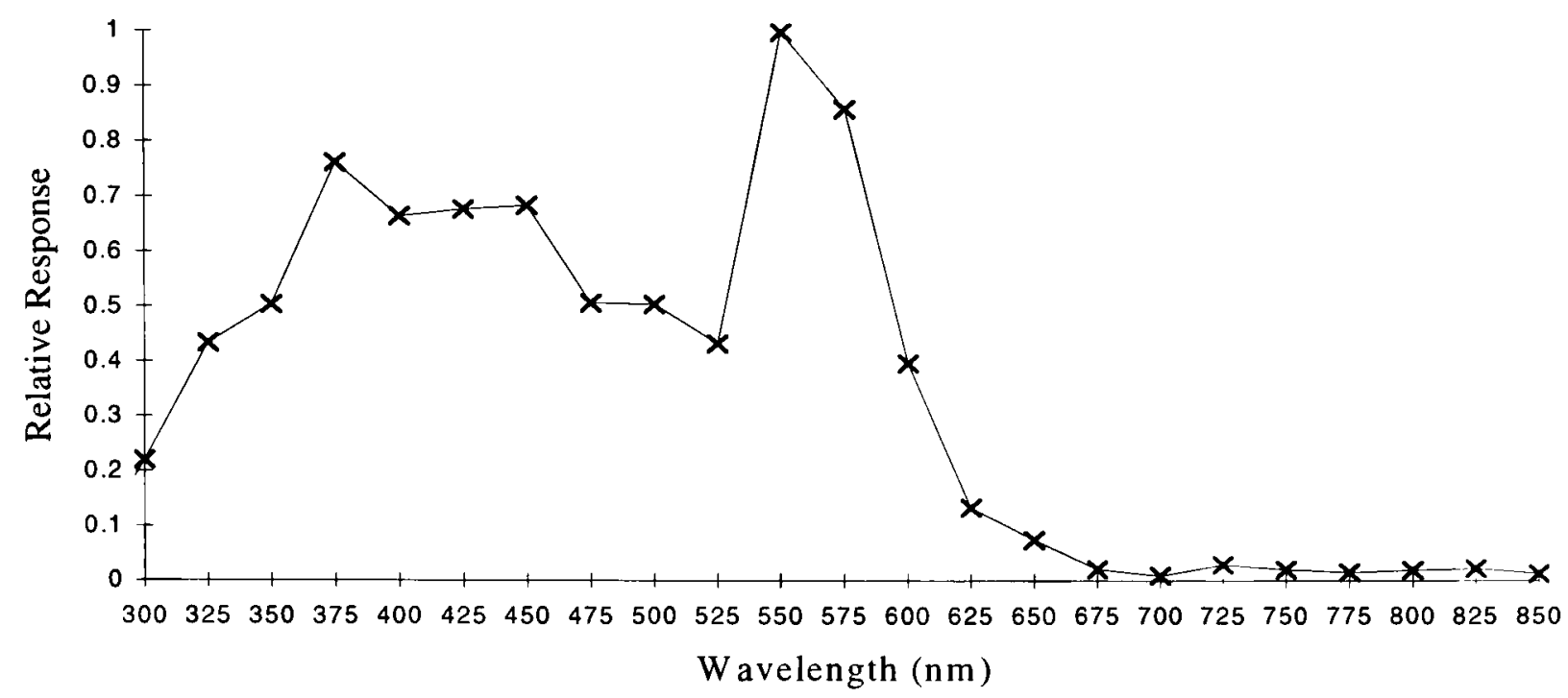

Figure 3-32 The mean wavelength sensitivity function for Papilio dardanus before calibration for lamp intensity.

The intensity sensitivity functions were all relatively smooth, gentle curves when plotted on a $\log$ scale (see Appendix 2). It was thus relatively easy to calculate the spectral efficiency function for Papilio dardanus at a constant (low) intensity (see Appendix 2 for details) - see Figure 3-33. It was also possible to plot the raw data as a 'sensitivity surface', plotting the relative response at varying intensities for each wavelength (see Figure 3-34), and to plot the intensity sensitivity functions (calculated in Appendix 2) for each wavelength to create an apparent surface (see Figure 3-35).

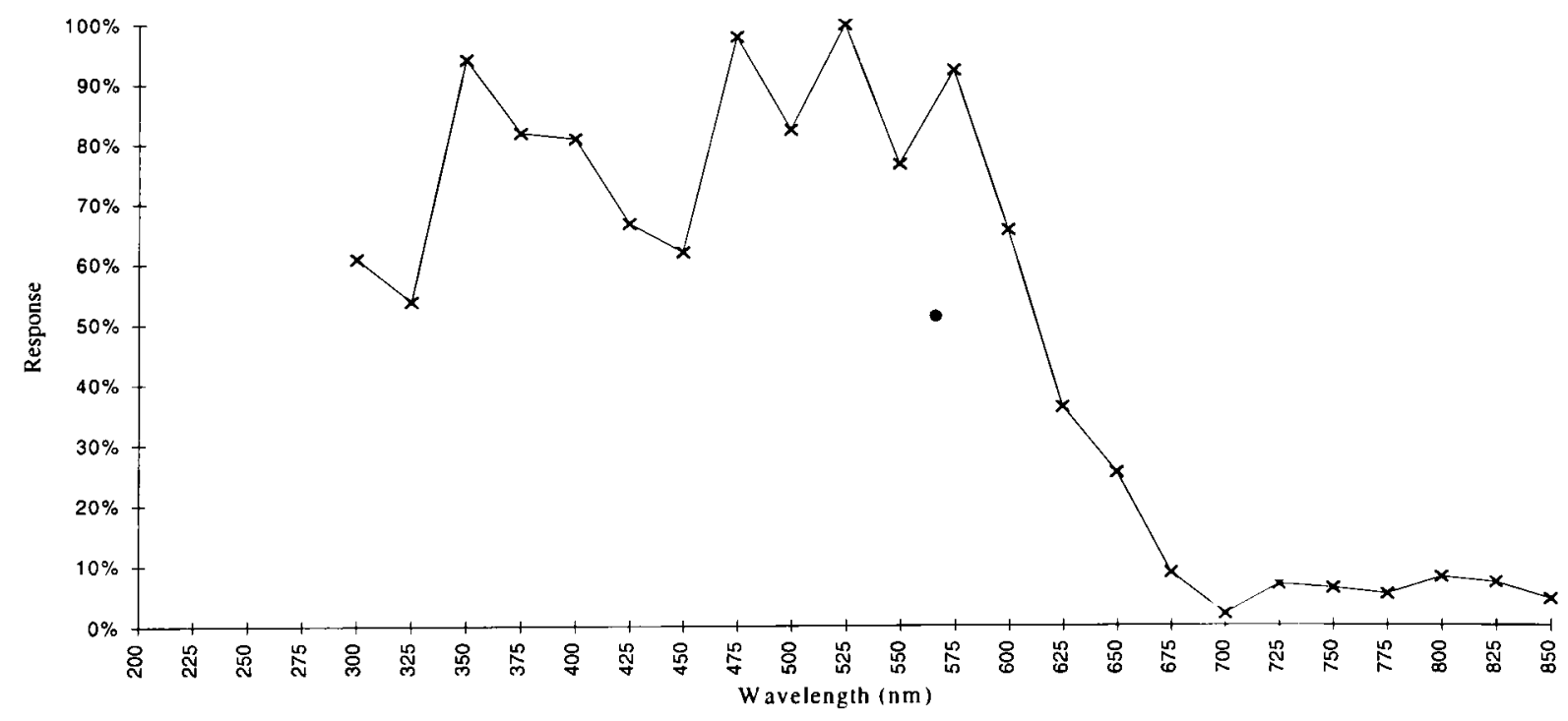

Figure 3-33 The spectral efticiency function for Papilio dardanus. 


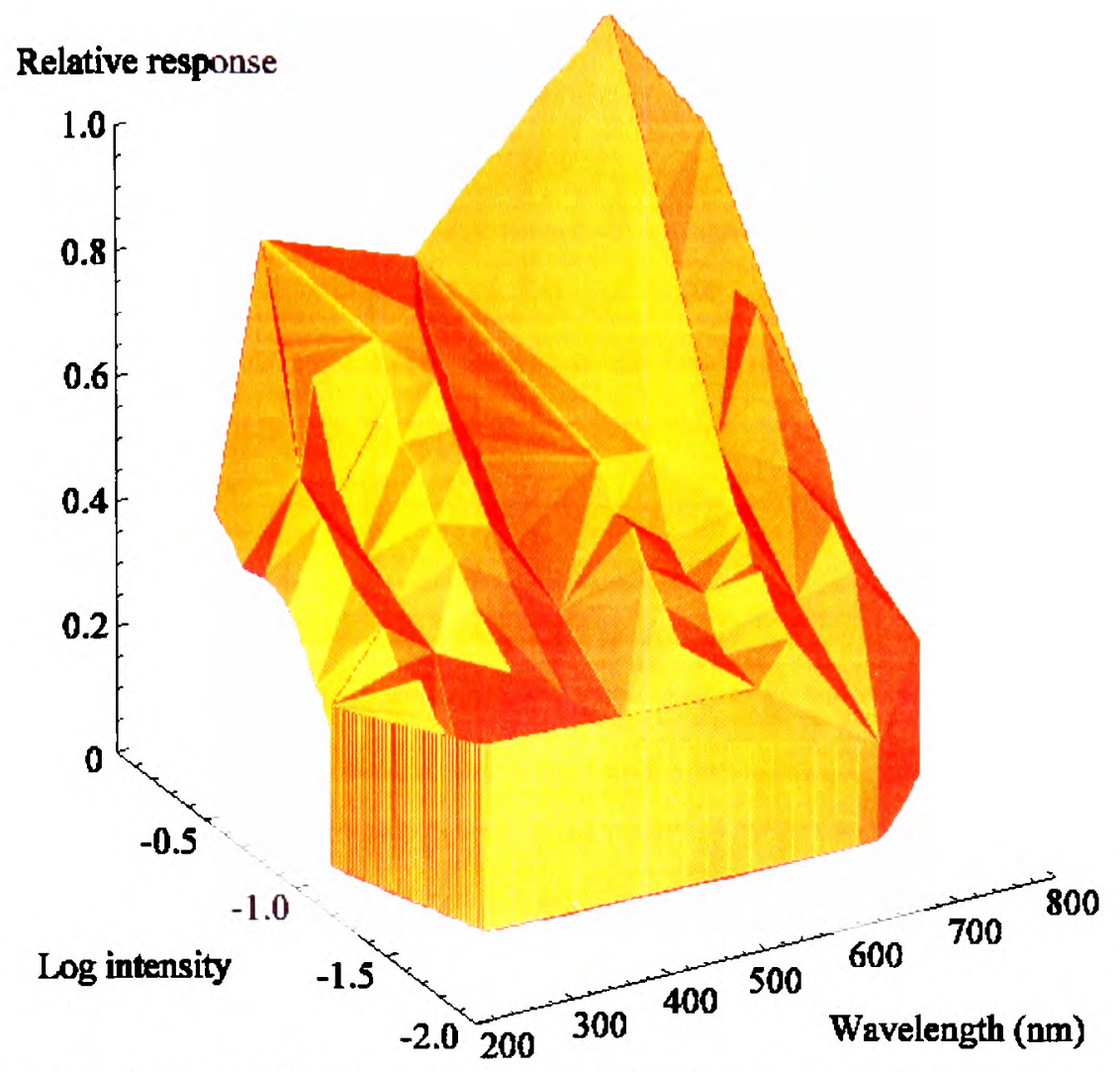

Figure 3-34 The raw ERG data plotted as a surface of relative response as a function of wavelength and intensity

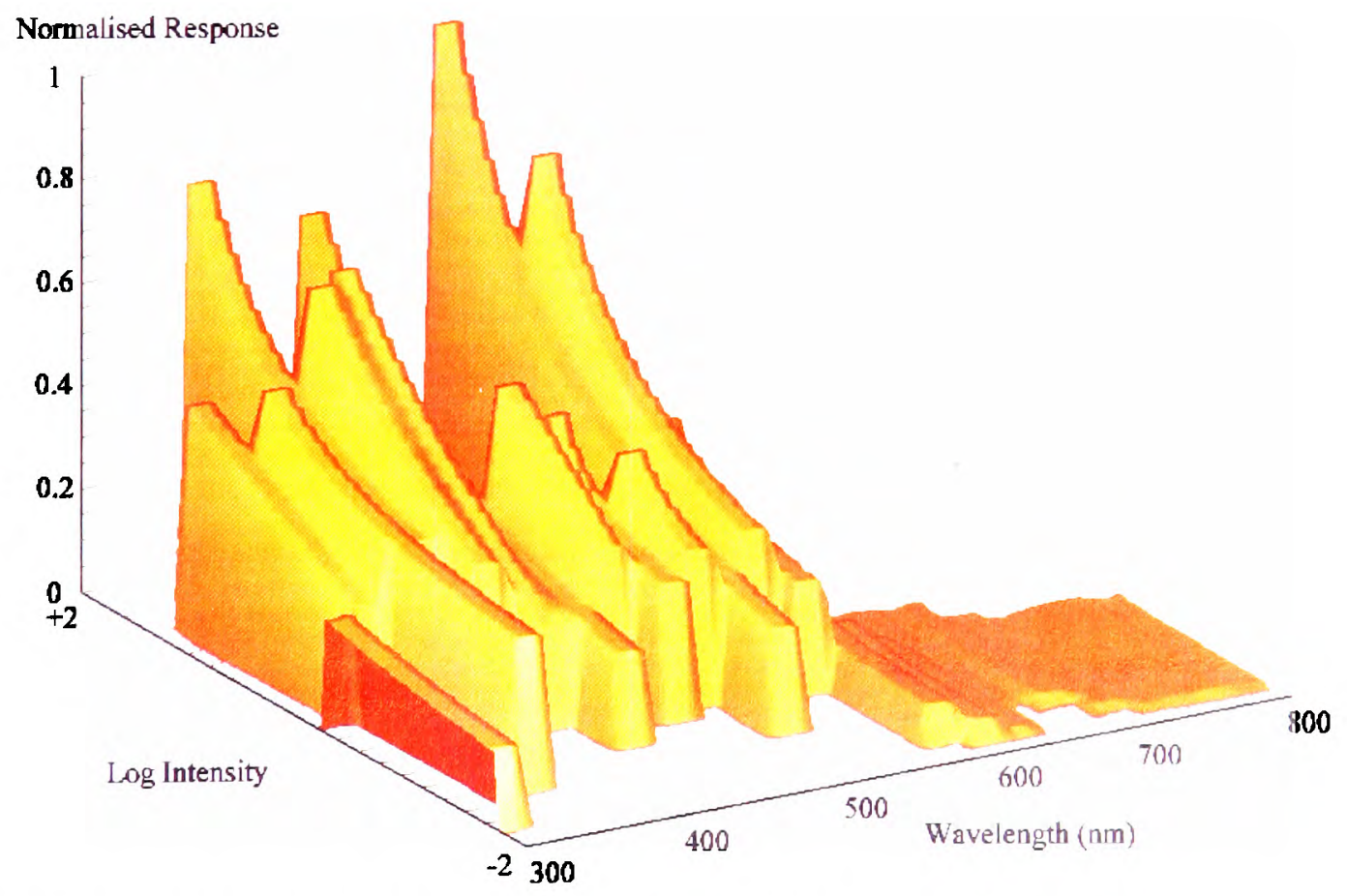

Figure 3-35 A plot of the interpolated intensity functions (from Appendix 2) for each wavelength 


\section{Discussion}

The spectral efficiency function for Papilio dardanus (at a constant intensity) shows peaks at $350 \mathrm{~nm}$ (UV), $475 \mathrm{~nm}$ (blue), 525nm (green), and 575nm (orange). These compare well with those reported for Papilio xuthus (Arikawa et al., 1987) which were found at $360 \mathrm{~nm}, 400 \mathrm{~nm}$ (no corresponding pigment found in Papilio dardanus), $460 \mathrm{~nm}, 520 \mathrm{~nm}$, and $600 \mathrm{~nm}$ (the Papilio dardanus peak in this area appears to be shifted slightly toward orange). The three main visual pigments found in insects are present, at approximately 360nm, 460nm, and 520nm (Chittka \& Menzel, 1992; Backhaus \& Menzel, 1992; Bennett et al., 1997; Bernard \& Remington, 1991).

How this measured output from the retina is interpreted by the butterflies is a matter of debate. In true 'colour vision', the output from the receptors containing visual pigments would be compared with each other, and the perceived colour would be a weighted average of the intensity received by each. Hence humans perceive purple when red and blue receptors are stimulated, and the perceived purple tends to red as comparatively more red or less blue is received. The alternative method of interpreting the output of the receptors would be for the butterflies to show 'wavelength-specific behaviour', where the receptor outputs are independently assessed, and each simply triggers a certain behaviour once it reaches a threshold of stimulation. Scherer \& Kolb (1987) performed experiments designed to test for the presence of true colour vision in the butterflies Aglais urticae and Pararge aegeria. They found that the butterflies did indeed appear to have true colour vision (although they also showed wavelength-specific behaviour). Therefore it seems likely that Papilio dardanus would have similar colour information processing, and this experiment indicates that they would have at least as good a colour vision as humans in the Visible range of the spectrum, and also possess good UV sensitivity.

The peaks in the Visible spectrum correspond to the wavelengths reflected well by Papiliochrome II (445-800 nm), but the UV peak does not quite correspond to the peak reflectance by Papiliochrome II (which is about 320nm) (See Figure 3-10). The butterflies will, however, still be able to detect the UV component well, and so the overall colour of the pigment will appear slightly shifted toward the blue end of the spectrum to them, compared with how we perceive it which make make it less obvious against the vegetation, as discussed in Experiment 3-3. The reflectance spectra of the white patches on hippocoonides (and Amauris niavius) show high reflectivity over all the spectrum between $360 \mathrm{~nm}-800 \mathrm{~nm}$, and therefore will appear true white to the butterflies, and distinguishable from the background vegetation by the presence of reflectance in the red/orange region of the spectrum as well as by a difference in brightness. The orange pigment has a reflectance spectrum which corresponds very well with the upper peaks in sensitivity, and has no UV component, so will appear reddish to the butterflies, also contrasting in hue from the background. 
The intensity-wavelength sensitivity surfaces (plotted in Figure 3-34 and Figure 3-35) show the general trend of greater response to greater intensities, but as the data is very incomplete (due to the need for higher intensities at certain wavelengths than the lamp was able to produce) it is difficult to interpret.

The results of this experiment will be very important in interpreting any colour preferences indicated by work in Chapter 4 (on the colour preferences of Papilio dardanus whilst feeding) and Chapter 5 (on mate choice in Papilio dardanus).

\section{Experiment 3-7 Fading of Papiliochrome II}

\section{Introduction}

Cook et al. (1994) described how the older males and trimeni females start to fade to an orange colour, apparently due to the action of sunlight, and Cockayne (1924) described how the yellow pigment in Papilionids "readily changes to a browner tint and becomes non-fluorescent". In Experiment 3-3 the colour change was demonstrated to involve a loss of green reflectance as well as fluorescence, and in addition the scales tended to lose their UV reflectance (which was shown to be a structural phenomenon in Experiment 3-5). Umebachi (1961) named the yellow pigment Papiliochrome II (which occurs together with very small quantities of a pigment he named Papiliochrome III), one of the Papiliochrome pigments unique to the Papilionid butterflies. The pigment is said to be broken down by heat and weak ammonia solution (Umebachi \& Yoshida, 1970), but there is no description of chemical change under the action of light.

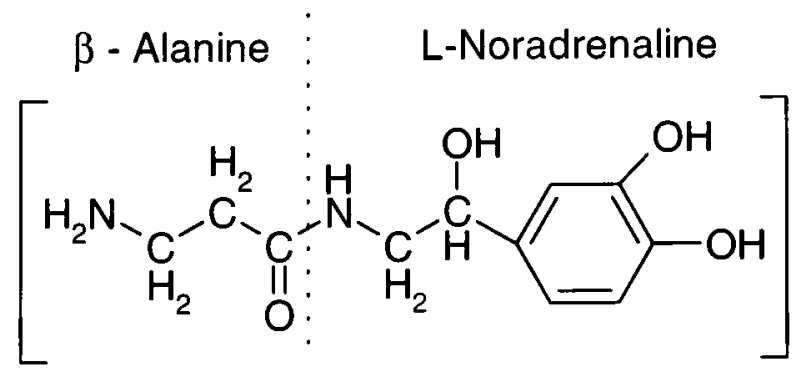
- L-Kynurenine<smiles>Nc1ccccc1C(=O)CC(N)C(=O)O</smiles>

Figure 3-36 Partial chemical structure of Papiliochrome II (after Kayser, 1985)

The fact that older individuals may gradually change colour introduces the interesting possibility of an age-cue in the species (Cook et al., 1994). This might be used by females in choosing a mate. A preference for younger males, judged by the extent of wing damage, has been found in 
Colias eurytheme (Rutowski, 1985), and this could be explained as a way of choosing more fertile partners as it has been shown in swallowtails that ejaculate mass decreases very rapidly in males (Svärd \& Wicklund, 1986).

It would be very interesting to do mate choice experiments to investigate whether or not females discriminate between males on the basis of their colour, and in order to do this with live males, it would be necessary to accelerate the fading process to compare males which differ in their colour but not in their actual age (so as not to give any other cues to the females which might affect their choice). If the fading of the pigment is caused by UV light, of which there is comparatively little in sunlight (Robinson, 1966) it might be possible to accelerate this process using an Ultraviolet lamp which produces a much higher intensity of the wavelengths responsible for the fading.

\section{Aim}

The aim of this experiment is to identify the wavelengths which cause the colour change of Papiliochrome II.

\section{Method \& Materials}

The wings of freshly dead male Papilio dardanus were pinned out in a glass greenhouse (to protect them from rain and wind). In each case one wing was completely protected from light, and another wing was half covered by a filter. The wings were left until the area completely exposed to the sunlight showed distinct fading (38 days between the $18^{\text {th }}$ May and $25^{\text {th }}$ June 1998). Both wings were then scanned together, using a HP Deskscan II, to ensure the same brightness and contrast settings.

Five different filters were used:

1) A UV filter (Image Optics Components ${ }^{*}$ ) which removed all wavelengths shorter than $400 \mathrm{~nm}$ (see Figure 3-37).

2) A red filter (Lee Filters ${ }^{* *}$ ) which removed all wavelengths shorter than $575 \mathrm{~nm}$ (see Figure 3-39).

3) A yellow filter (Lee Filters) which removed all wavelengths shorter than $475 \mathrm{~nm}$ (see Figure 3-38).

4) A green filter (Lee Filters) which removed wavelengths between 600 and $700 \mathrm{~nm}$ and had limited transmission to other wavelengths (see Figure 3-41).

5) A blue filter (Lee Filters) with a similar spectrum, but slightly higher transmission to other wavelengths (see Figure 3-40). 
(Spectra for red, yellow, green and blue filters obtained from manufacturer, spectra for UV filter obtained using UV-Vis spectrometer)

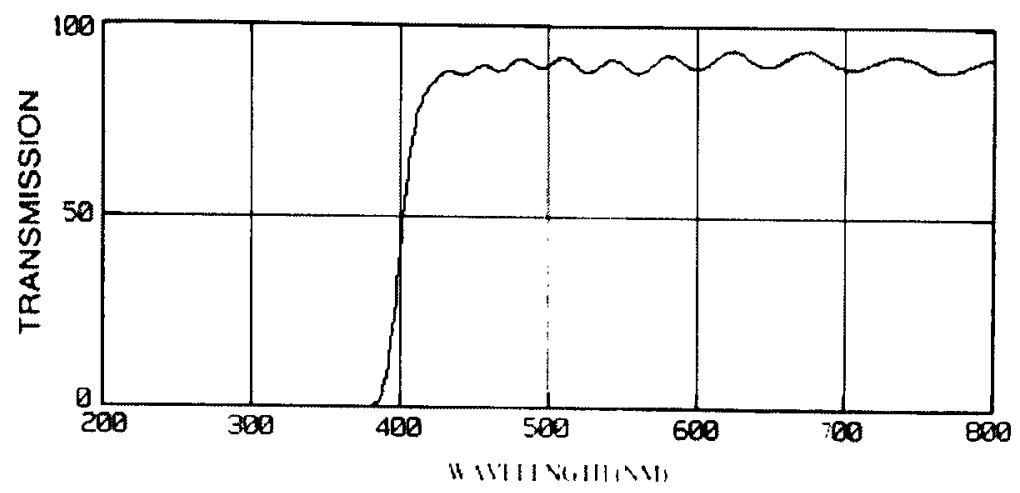

Figure 3-37 The transmission spectrum of the UV filter

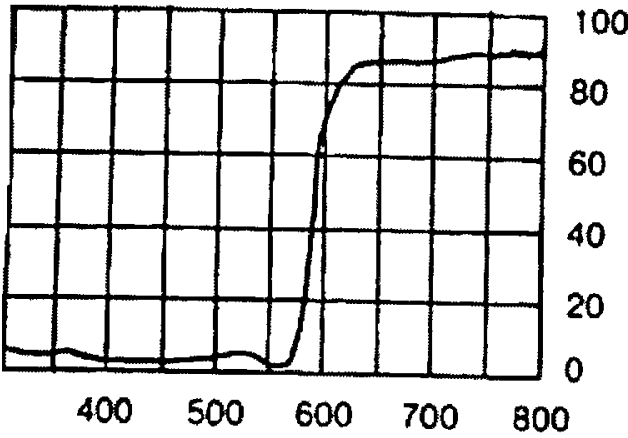

WHELEVGTHINH

Figure 3-39 The transmission spectrum of the red filter

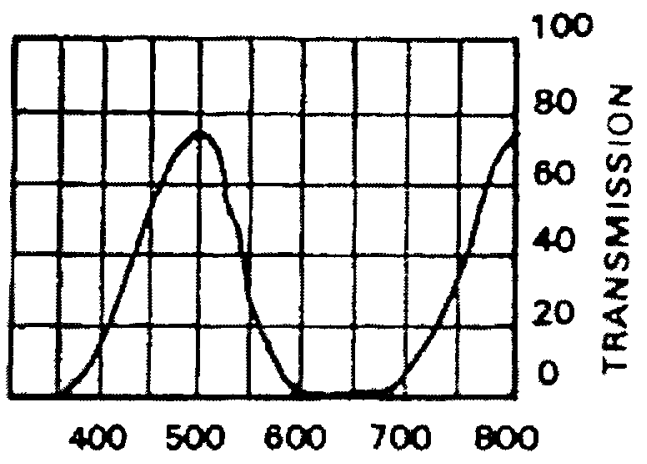

16. AVELENGTH(NWH
Figure 3-41 The transmission spectrum of the green filter

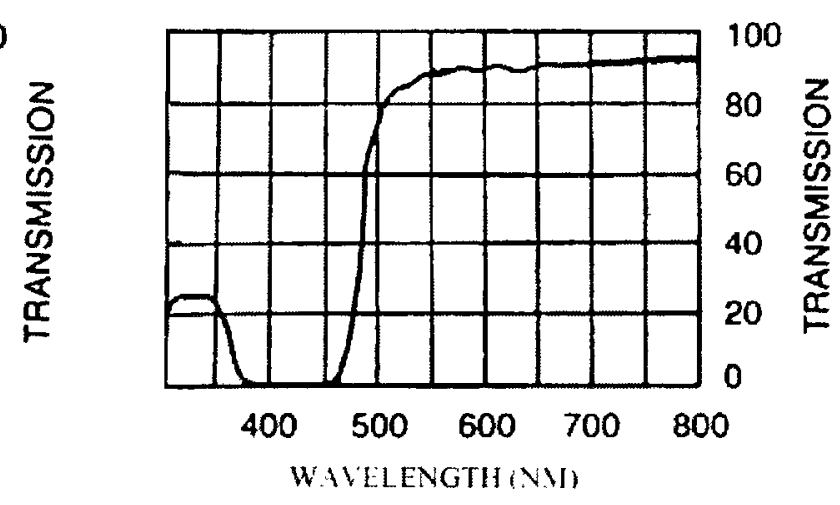

Figure 3-38 The transmission spectrum of the yellow filter

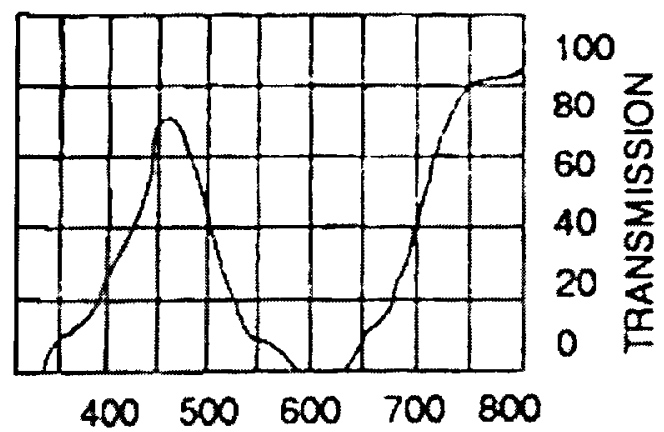

II IVFIE $\backslash$ GTHINI)

Figure 3-40 The transmission spectrum of the blue filter

\section{Results}

1) The UV filter did not prevent fading of the pigment (see Figure 3-42, filtered region of right wing), therefore the UV component of sunlight cannot be responsible for the process. The very small difference in colour between the exposed and filtered parts of the right hand wing could be due to the fact that the filter also removes about $5-10 \%$ of the intensity of all other wavelengths (see Figure 337). 


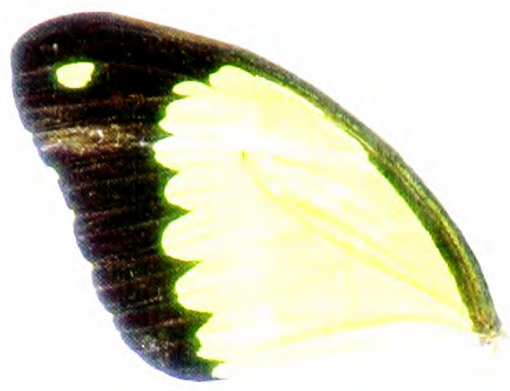

Covered

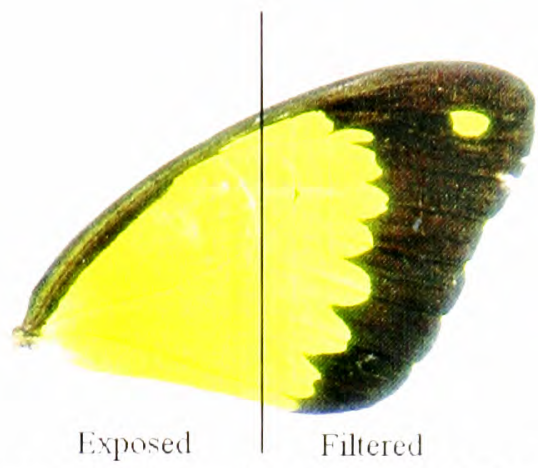

Figure 3-42 The effect of removing the UV component of sunlight, using a filter, on the fading of Papiliochrome II.

2) The filter removing wavelengths shorter than $575 \mathrm{~nm}$ appeared to prevent fading altogether (see Figure 3-43). This indicates that the wavelengths responsible for the fading are shorter than $575 \mathrm{~nm}$, but the result from the UV filter indicate that they are longer than $400 \mathrm{~nm}$.
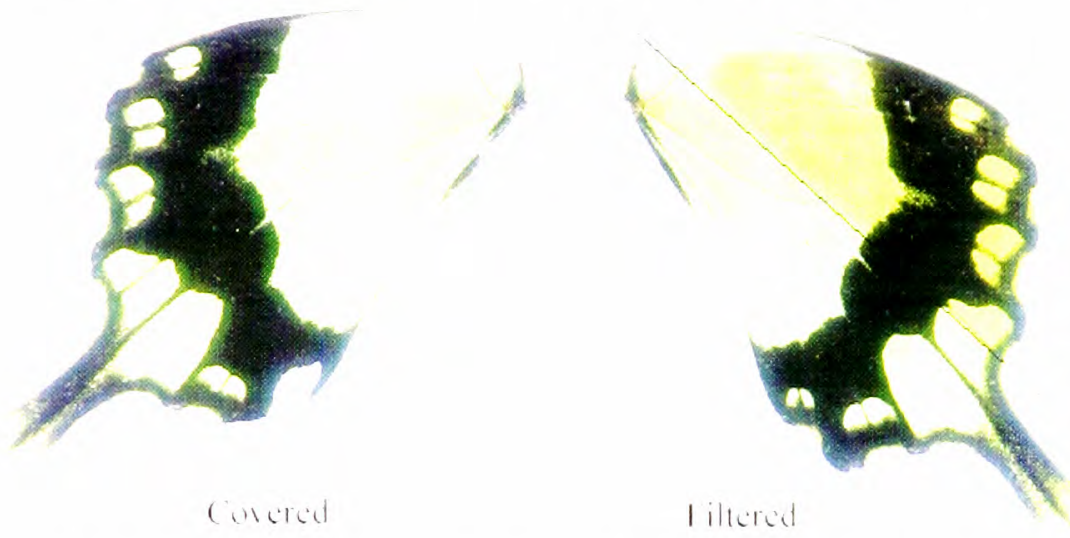

Figure 3-43 The effect of removing wavelengths shorter than $575 \mathrm{~nm}$ on the fading of Papiliochrome II

3) The filter removing wavelengths shorter than $475 \mathrm{~nm}$ also prevented any fading from occurring (see Figure 3-44). This indicates that the wavelengths responsible for the change are shorter than $475 \mathrm{~nm}$, and it is already known from the result of applying a UV filter that they are longer than $400 \mathrm{~nm}$.
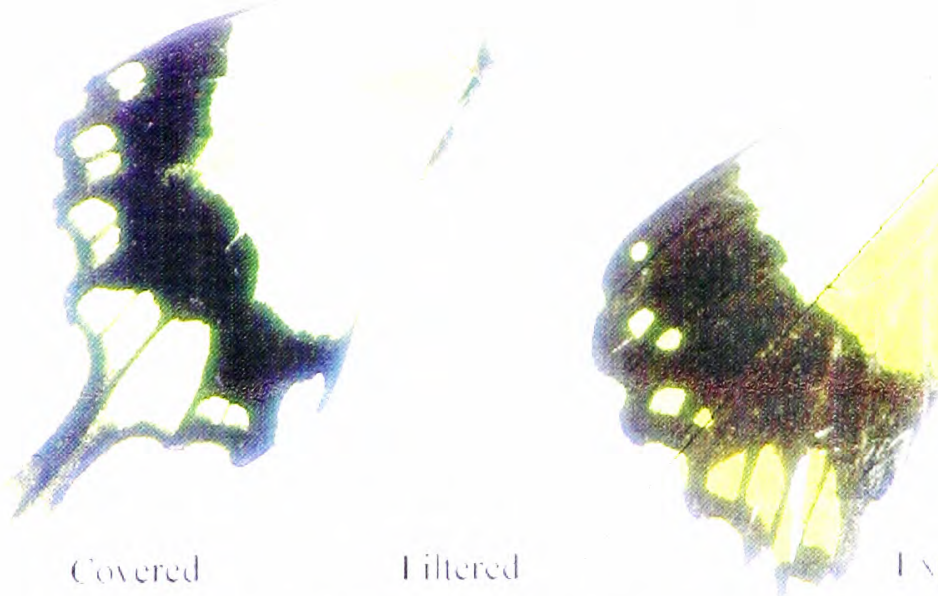

Figure 3-44 The effect of removing wavelengths shorter than $475 \mathrm{~nm}$ on the fading of Papiliochrome II 
4) The green filter, as would be expected from the results above, allowed some fading of the pigment (see Figure 3-45)

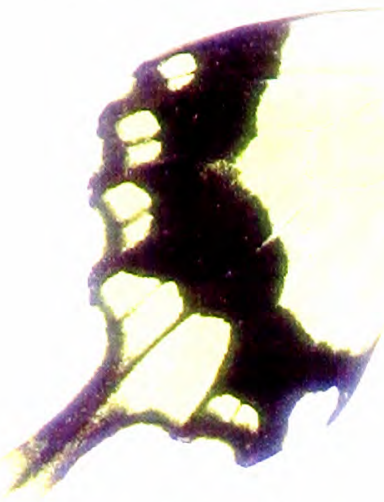

Covered

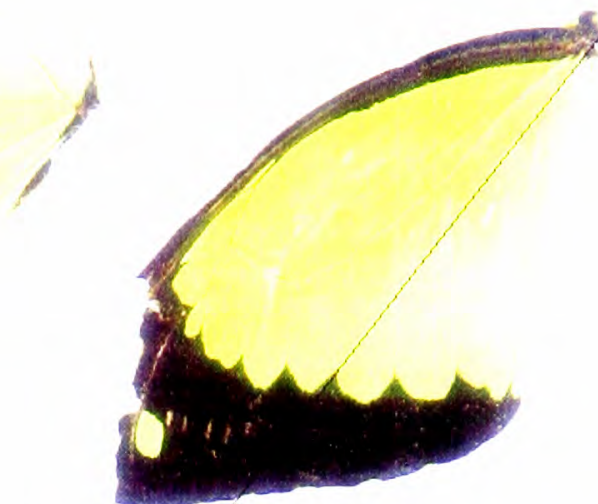

Lxposed

filtered

Figure 3-45 The effect of a green filter on the fading of Papiliochrome II

5) The blue filter, with a transmission spectrum which was virtually identical to the green filter in the region $400-475 \mathrm{~nm}$, gave a very similar result to the green filter as might be expected, and the two are not distinguishable (see Figure 3-46).

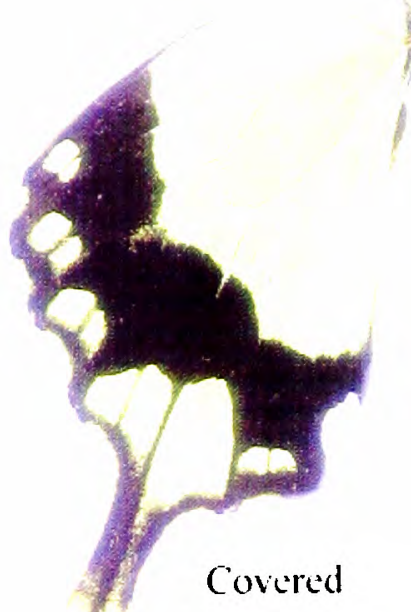

Covered

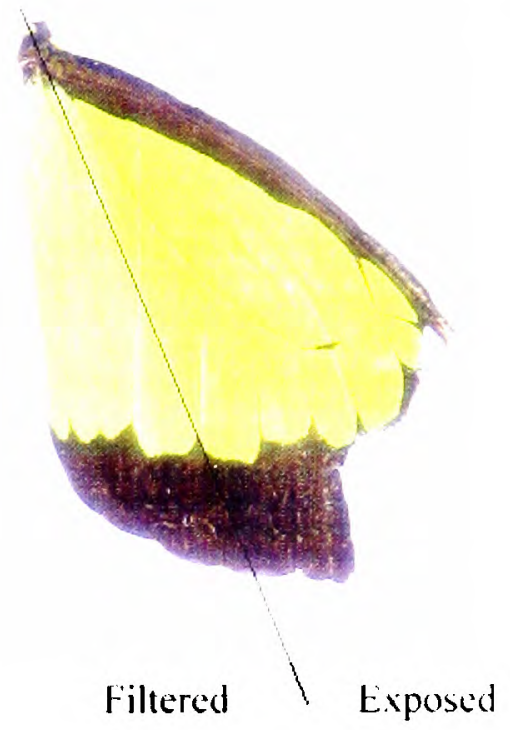

Figure 3-46 The effect of a blue filter on the fading of Papiliochrome II

It can therefore be seen that removing the wavelengths shorter than $400 \mathrm{~nm}$ (UV) has no effect on the fading of Papiliochrome II, while removing the wavelengths shorter than $475 \mathrm{~nm}$ (green/blue/UV) prevents its fading. It can therefore be concluded that the wavelengths responsible are between 400 and $475 \mathrm{~nm}$. This results in some fading being seen with the blue and green filters which allow some limited transmission in these regions.

\section{Discussion}

This experiment indicates that the wavelengths responsible for the colour change in the yellow Papiliochrome II pigment of Papilio dardanus lie between 400 and $475 \mathrm{~nm}$, and are therefore in the 
blue/violet rather that the UV region of the spectrum. It is therefore not possible to cause artificially rapid fading in live butterflies by using a UV bulb in order to create 'apparently old' males for use in mate choice experiments. If a bulb with a suitably strong illumination in the $400-500 \mathrm{~nm}$ region of the spectrum could be used to fade live specimens, mate choice experiments using them could provide very interesting data on whether or not the females use the colour change of older males as an age-cue when selecting mates. Similar experiments could be carried out on the effect that such illumination of andromorphic females had on male choice. Since such a bulb could not be found, these experiments could not be carried out.

\section{Conclusions}

The photographs from Experiment 3-1 demonstrate that the patterning of the morphs of Papilio dardanus is likely to be unchanged by different spectral sensitivities of the signal receivers. The reflectance spectra from Experiment 3-3 (and the knowledge, from Experiment 3-2, that the fluorescence of the yellow pigment is so relatively weak) indicate how the colours might be seen by receivers with varying sensitivities, and the ERG data from Experiment 3-6 gives an idea of the sensitivities of the butterflies themselves, and hence allows an assessment of the signals they might be receiving from each other. If the butterflies have true colour vision, as has been shown in other species (Scherer \& Kolb, 1987) the UV reflectancy of the yellow scales would shift the perceived colour of the patches slightly toward the blue end of the spectrum for the butterflies compared with how they appear to us, and thus the spectral contrast between the wings and the non-UV reflectant vegetation (Klein, 1978) could be much lower than we perceive it to be. This means that the yellow need not necessarily be obvious to the butterflies themselves. The true-white patches of hippocoonides, however, are likely to be very obvious against the background vegetation. The orange colour, having no UV component, should appear in relatively the same area of the spectrum to the butterflies as it does to us.

The reflectance spectra show that the white patches in the hippocoonides morph appear to have exactly the same reflective properties (including the UV range) as those in Amauris niavius, increasing the close resemblance of the model and mimic. The UV peak of the white patches in both species is $255 \mathrm{~nm}$, as opposed to one of $320 \mathrm{~nm}$ found in the yellow patches of Papilio dardanus (assumed to be the ancestral colour). The transmission electron microscopy has shown that this is due to a structural change in the width and spacing of the lamellae on the scale ridges. This demonstrates that hippocoonides is quite a sophisticated mimic of Amauris niavius, and that the mimicry was not due simply to a loss of yellow pigment from a male-like trimeni form. The fact that there appears to be evolutionary pressure for the hippocoonides to mimic Amauris niavius in this extreme range of the 
spectrum indicates that the main predators probably have spectral sensitivity in this region, and this should be investigated further.

The role of the orange pigment is a matter for debate. It seems that it has either appeared independently in both hippocoonides- and trimeni-like forms, or that it occurred firstly in a trimenilike form, and that the white, trophonius morph is subsequently derived from the yellow, prototrophonius morph which has not yet been totally excluded by the presence of the trophonius form.

There are two hypotheses to explain the presence of the lamborni morphs. Cook et al. (1994) suggested that for females trying to avoid 'sexual harassment' (which they suggest as a reason for the evolution of a male-mimicking trimeni form), there might be a selection pressure for loss of fluorescence and reflectance in order to mimic the older males (which they demonstrated to be approached even less often by males than younger males). However, this hypothesis does not explain why the orange pigmentation is so strictly limited to the hindwings and lower forewings - fully orange forms such as pale poultoni (found in race polytrophus from Kenya) would be much more likely mimics of faded males. It also does not explain the presence of white lamborni (trophonius) - why should a hippocoonides-like morph evolve an orange pigmentation over part of its wings, leaving white flashes on the forewings, disrupting its mimicry of Amauris niavius, and not being a good mimic of a faded male Papilio dardanus? The spectral similarity of the white patches in white lamborni and hippocoonides and the fact that this appears to be due to an alteration of the structure of the scales suggests that the white lamborni form is derived directly from hippocoonides, although without studying the spectrum of the white patches in Danaus chrysippus it is not possible to say that hippocoonides and white lamborni have not independently evolved the same coloration.

Some mainland trophonius morphs do resemble Danaus chrysippus very closely (Trimen, 1869), and it seems very likely that these are indeed mimics of Danaus chrysippus. According to the second hypothesis (Trimen, 1869 and subsequent authors) the yellow lamborni (prototrophonius) evolved first from a trimeni-like morph due to predator pressure causing a resemblance of Danaus chrysippus (although this first stage could have been due to a 'sexual harassment' pressure as suggested by Cook et al.). Following the evolution of yellow lamborni (prototrophonius), the evolution of white lamborni (trophonius) would simply be due to a pressure for a closer mimicry of Danaus chrysippus. The similarity of the spectra of hippocoonides and white lamborni appears to run counter to this proposed sequence of events, but as has been mentioned previously, the spectra of Danaus chrysippus need to be studied before all the evidence can be assessed.

Whatever the pressures involved, the presence of apparently non-functional lamellae in the orange scales of lamborni suggest that the orange pigment may have evolved relatively recently, and 
the fact that the lamborni or trophonius/prototrophonius morphs are dominant over all other morphs in the races in which they occur also suggest that they might have appeared most recently. It has been suggested that dominance over existing mimetic forms evolves in new mimetic forms (Sheppard, 1967), and a mathematical analysis of the situation has confirmed that in many cases this will be true, as a poorer mimic (as the first individuals of a new mimetic form will almost certainly be) will tend to become dominant over a better mimic of another model (Charlesworth \& Charlesworth, 1976). This result, however, is based on many assumptions, and cannot be held as a strong prediction, so the patterns of dominance within Papilio dardanus can only be regarded as suggestive of the patterns of evolution of the morphs.

The fact that the faded individuals (which would be older in life than those which had not had a chance to fade) had become very much less fluorescent and, more importantly, less UV reflectant (shifting their apparent colour towards red), means that they are likely to be clearly perceptible as older to potential mates. This may therefore be a mechanism by which females can choose between potential partners (see the introduction to Experiment 3-7). However, any male mutant which managed to retain its reflectance longer would leave more offspring (by obtaining matings later in life, even given decreased fertility), so it would seem likely that some means of retaining the original coloration would evolve if at all possible if the selection pressure is large enough. It is also conceivable that females may choose an older partner, who has proved his survival ability (despite the fact that he may have a lower viability due to previous matings). Again, however, it might be expected that males would have 'cheated' and evolved an orange pigment, possibly similar to that found in the female pale poultoni morphs if females preferred such orange coloration.

Mate choice experiments in this area are required, but as discussed in Experiment 3-7 it has proved very difficult to fade the males artificially, and hence males which are genuinely older than others would have to be used in order to gauge any preference. This will be discussed further in Chapter 5 . However, the information gained from this chapter on the signals actually produced by the different morphs of Papilio dardanus, and its visual sensitivities will prove invaluable in interpreting the results of the next two chapters, on the flower colour preferences and mate choices of the species.

\section{Acknowledgements}

I would like to thank Sandor Ertz at the British Antarctic Survey, Cambridge for the use of the spectrophotometer; Richard Compton for the use of the fluorescence spectrometer, chart recorder, mercury lamp and monochromator; Jenny Corrigan and Martin Lomas for help with the preparation and viewing of the electron microscopy samples; and the Zoology Department Photographic Unit for 
the development and printing of the photographs. I am also very grateful to Steve Simpson and John Alden for help with the ERG measurements; and to Pauline Rigby for introducing me to the Multilayer software.

\section{References}

Anderson, T.F. \& Richards, A.G. 1942. An electron microscope study of some structural colours of insects. J. Appl. Phys. 13, 748-58.

Arikawa, K., Inokuma, K., Eguchi, E. 1987. Pentachromatic visual system in a butterfly. Naturwissenschaften 74, 297-298.

Autrum, H. 1958. Electrophysiological analysis of the visual systems in insects. Expl. Cell Res. (suppl.) 5, 426-439.

Backhaus, W. \& Menzel, R. 1992. Conclusions from the colour vision of insects. Behavioural and Brain Sciences 15 (1), 28-30.

Bennett, R.R., White, R.H., Meadows, J. 1997. Regional specialisation in the eye of the sphingid moth Manduca sexta: blue sensitivity of the ventral retina. Visual Neuroscience 14, 523-526.

Bernard, G.D. 1979. Red-absorbing visual pigment of butterflies. Science 203 (1), 1125-7.

Bernard, G.D. \& Remington, C.L. 1991. Color vision in Lycaena butterflies: spectral tuning of receptor arrays in relation to behavioural ecology. Proc. Natl. Acad. Sci. USA 88, 2783-2787.

Bernardi, G. 1963. Quelques aspects zoogéographiques du mimétisme chez les Lépidpidoptères. Proceedings, $16^{\text {th }}$ International Congress of Zoology, Washington 4, 161-166.

Bingham, L., Bingham, S., Geary, S., Tanner, J., Driscoll, C., Cluff, B., Gardner, J.S. 1995. SEM Comparison of Morpho butterfly dorsal and ventral scales. Microscopy Research and Technique 31, 93-4.

Brunton, C.F.A. \& Majerus, M.E.N. 1995. Ultraviolet colours in butterflies - intraspecific or interspecific communication? Proc. Roy. Soc. Lond. B 260 (1358), 199-204.

Carpenter, G.D.H. 1941. Observations and experiments in Africa by the late C.F.M. Swynnerton on wild birds eating butterflies and the preference shown. Proc. Linn. Soc. Lond. 154, 10-46.

Charlesworth, D. \& Charlesworth, B. 1976. Theoretical genetics of Batesian mimicry III Evolution of dominance. J. Theor. Biol. 55, 325-337.

Chen, D-M. \& Goldsmith, T.H. 1986. Four spectral classes of cone in the retinas of birds. J. Comp. Physiol. A 159, 473-479.

Chittka, L. \& Menzel, R. 1992. The evolutionary adaptation of flower colours and the insect pollinators' colour vision. J. Comp. Physiol. A 171, 171-181.

Clarke, C.A., Clarke, F.M.M., Collins, S.C., Gill, A.C.L., Turner, J.R.G. 1985. Male-like females, mimicry and transvestism in swallowtail butterflies. Systematic Entomology 10, 257-283. 
Clarke, C.A., Gordon, I.J., Vane-Wright, R.I., Smith, C.R. 1991. Phylogenetic relationships of three African swallowtail butterflies, Papilio dardanus, $P$. phorcas and $P$. constantinus: new data from hybrids (Lepidoptera: Papilionidae). Systematic Entomology 16, 257-273.

Clarke, C.A. \& Sheppard, P.M. 1963. Interactions between major genes and polygenes in the determination of the mimetic patterns of Papilio dardanus. Evolution 17, 404-413.

Cook, S.E., Vernon, J.G., Bateson, M., Guilford, T. 1994. Mate choice in the polymorphic African swallowtail butterfly, Papilio dardanus - male-like females may avoid sexual harassment. Animal Behaviour 47 (2), 389-397.

Cockayne, E.A. 1924. The distribution of fluorescent pigments in the Lepidoptera. Trans. Entomol. Soc. London, 1-19.

Eguchi, E., Watanabe, K., Hariyama, T., Yamamoto, K. 1981. A comparison of electrophysiologically determined spectral responses in 35 species of Lepidoptera. J. Insect Physiol. $28(8), 675-682$.

Ford, E.B. 1936. The genetics of Papilio dardanus Brown (Lep.). Transactions of the Royal Entomological Society of London 85. 435-466.

Ghiradella, H., Aneshansley, D., Eisner, T., Silberglied, R.E., Hinton, H.E. 1972. Ultraviolet reflection of a male butterfly: Interference color caused by thin-layer elaboration of wing scales. Science 178, 1214-1217.

Ghiradella, H. 1974. Development of ultraviolet-reflecting butterfly scales: How to make an interference filter. Journal of Morphology 142, 395-410.

Ghiradella, H. \& Radigan, W. 1976. Development of butterfly scales, 2: Struts, lattices, and surface tension. Journal of Morphology 150, 279-298.

Ghiradella, H. 1984. Structure of iridescent lepidopteran scales: Variations on several themes. Annals of the Entomological Society of America 77, 637-645.

Ghiradella, H. 1985. Structure and development of iridescent lepidopteran scales: The Papilionidae as a showcase family. Annals of the Entomological Society of America 78, 252-264.

Ghiradella, H. 1989. Structure and development of iridescent butterfly scales: Lattices and laminae. Journal of Morphology 202, 69-88.

Ghiradella, H. 1991. Light and colour on the wing - structural colours in butterflies and moths. Applied Optics 30 (24), 3492-3500.

Ghiradella, H. 1994. Structure of butterfly scales: Patterning in an insect cuticle. Microscopy Research and Technique 27, 429-438.

Kayser, H. 1985. Pigments. In Comprehensive insect physiology, biochemistry and pharmacology Vol. 10 ed. Kerkut, G.A. \& Gilbert, L.I., 367-415. Pergamon, Oxford.

Kevan, P., Giurfa, M., Chittka, L. 1996. Why are there so many and so few white flowers? Trends in Plant Sciences 1(8), 280-284.

Klein, R.M. 1978. Plants and near-ultraviolet radiation. Bot. Rev. 44, 1-127.

Land, M.F. 1972. The physics and biology of animal reflectors. Progress in Biophysics and Molecular Biology 24, 77-123. 
Lippert, W. \& Gentil, K. 1959. Über lamellare Feinstrukturen bei den Schillerschuppen der Schmetterlinge vom Urania and Morpho-Typ. Z. Morph. Ökol. Tiere 48, 115-122.

Mason, C. 1926. Structural colours in insects, 1. J. Phys. Chem. 30, 383-395.

Mason, C. 1927a. Structural colours in insects, 2. J. Phys. Chem. 31, 320-354.

Mason, C. 1927b. Structural colours in insects, 3. J. Phys. Chem. 31, 1856-1872.

Menzel, R. 1979. Spectral sensitivity and colour vision in invertebrates. In Handbook of Sensory Physiology Vol. VIV/6A "Comparative physiology and evolution of vision in invertebrates" ed. Autrum, H., 503-580. Springer, Berlin.

Nijhout, H.F. 1985. The developmental physiology of colour patterns in Lepidoptera. Advances in Insect Physiology 18, 181-247.

Nijhout, H.F. 1991. The development and evolution of butterfly wing patterns. Smithsonian Institute Press.

O'Donald, P. \& Barrett, J.A. 1973. Evolution of dominance in polymorphic Batesian mimicry. Theoretical Population Biology 4, 173-192.

Poulton, E.B. 1924. Papilio dardanus. The most interesting butterfly in the world. Journal of the East African and Ugandan Natural History Society 20, 4-22.

Robinson, N. 1966. Solar Radiation. Amsterdam, Elsevier.

Rutowski, R.L. 1985. Evidence for mate choice in a sulphur butterfly (Colias eurytheme). $Z$. Tierpsychol. 70, 103-114.

Scherer, C \& Kolb, G. 1987. The influence of color stimuli on visually controlled behavior in Aglais urticae L. and Pararge aegeria L. (Lepidoptera). J. Comp. Physiol. A 161, 891-898.

Sheppard, P.M. 1967. Natural selection and heredity ( $3^{\text {rd }}$ edn.). Hutchinson, London.

Silberglied R.E. 1979. Communication in the Ultraviolet. Ann. Rev. Ecol. Syst. 10, 373-398.

Svärd, L. \& Wiklund, C. 1986. Different ejaculate delivery strategies in first versus subsequent matings in the swallowtail butterfly Papilio machaon L.. Behav. Ecol. Sociobiol. 18, 325-330.

Swihart, C.A. 1971. Colour discrimination by the butterfly, Heliconius charitonius Linn. Anim. Behav. 19, 156-164.

Swihart, S.L. \& Gordon, W.C. 1971. Red photoreceptor in butterflies. Nature 231, 126-127.

Trimen, R. 1869. On some remarkable mimetic analogies among African butterflies. Transactions of the Linnean Society of London 26, 497-522.

Turner, J.R.G. 1963. Geographical variation and evolution in the males of the butterfly Papilio dardanus Brown (Lepidoptera: Papilionidae). Transactions of the Royal EntomologicalSociety of London 115, 239-259.

Umebachi, Y. 1961. Yellow pigments in the wings of the papilionid butterflies - V. Some chemical properties of the yellow pigments of Papilio xuthus. Sci. Rep. Kanazaw'a Univ. 7, 139-150. 
Umebachi, Y. \& Yoshida, K. 1970. Some chemical and physical properties of Papiliochrome II in the wings of Papilio xuthus. J. Insect Physiol. 16, 1203-1228.

Van Bemmelen, J.F. 1922. The wing-design of mimetic butterflies. Proceedings, Section of Sciences, Koninklijke Nederlandse Akademie Wetensschappen 23, 877-886.

Vane-Wright, R.I., Raheem, D.C., Cieslak, A., Vogler, A.P. In press. Evolution of the mimetic African swallowtail butterfly Papilio dardanus: molecular data confirm relationships with $P$. phorcas and P. constantinus. Biol. J. Linn. Soc.

Vane-Wright, R.I. \& Smith, C.R. 1991. Phylogenetic relationships of three African swallowtail butterflies, Papilio dardanus, $P$. phorcas, and $P$. constantinus: a cladistic analysis (Lepidoptera: Papilionidae). Systematic Entomology 16: 275-291. 


\section{Chapter Four}

\section{Flower choice in Papilio dardanus}

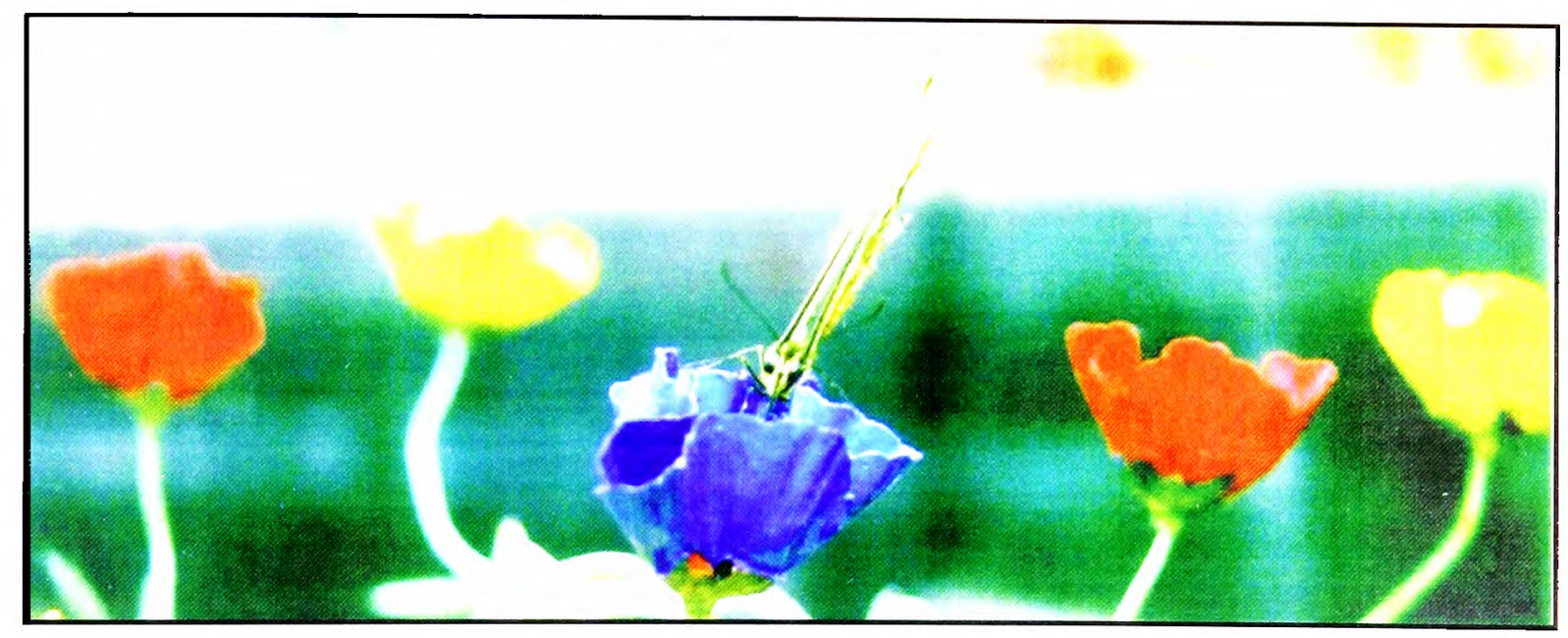

Figure 4-1 A male Papilio dardanus feeding off a blue artificial flower in Experiment 4-1

"Bees and various other insects must be directed by instinct to search flowers for nectar and pollen, as they act in this manner without instruction as soon as they emerge from the pupa state"

Charles Darwin (1877, Chap XI, p.425) 


\section{Introduction}

There is a wide range of flowers available to nectar-feeding insects, and the range changes with time. Studies on bees (von Frisch, 1914) first showed that such insects can show innate colour preferences which can subsequently be changed by learning, and similar experiments on various butterfly species have shown that they also have innate preferences (see Table 4-1), and show a considerable degree of flower constancy (Goulson \& Cory, 1993).

\begin{tabular}{|c|c|c|}
\hline Species & Colour preference & Reference \\
\hline Heliconius charitonius & $\begin{array}{l}\text { primary: orange/red } \\
\text { secondary: blue/green }\end{array}$ & $\begin{array}{l}\text { Swihart \& Swihart (1970) } \\
\text { Swihart (1971) }\end{array}$ \\
\hline Battus philenor & $\begin{array}{l}\text { primary: yellow } \\
\text { secondary: blue/purple }\end{array}$ & Weiss (1997) \\
\hline Aglais urticae & $\begin{array}{l}\text { blue/green \& yellow/red } \\
\text { primary: } 460 \mathrm{~nm} \\
\text { secondary: } 582 \mathrm{~nm}\end{array}$ & $\begin{array}{l}\text { Ilse (1928) } \\
\text { Scherer \& Kolb (1987) }\end{array}$ \\
\hline Pararge aegeria & $\begin{array}{l}\text { primary: } 450 \mathrm{~nm} \\
\text { secondary: } 630 \mathrm{~nm}\end{array}$ & Scherer \& Kolb (1987) \\
\hline Heliconius erato hydara & yellow/orange & Crane (1955) \\
\hline $\begin{array}{l}\text { 'tropical Papilionids and } \\
\text { Pierids' }\end{array}$ & $\begin{array}{l}\text { red (observation in field } \\
\text { only) }\end{array}$ & Crane (1955) \\
\hline Pieris brassicae & $\begin{array}{l}\text { orange/red \& blue/purple } \\
\text { primary: } 447 \mathrm{~nm} \\
\text { secondary: } 600 \mathrm{~nm}\end{array}$ & $\begin{array}{l}\text { Ilse (1928) } \\
\text { Scherer \& Kolb (1987) }\end{array}$ \\
\hline Pieris rapae & $\begin{array}{l}\text { primary: purple/blue } \\
\text { secondary: yellow } \\
\text { Some individuals flower- } \\
\text { constant, whilst others not. }\end{array}$ & Kandori \& Ohsaki (1996) \\
\hline Pieris napi & $\begin{array}{l}\text { no difference between } \\
\text { number with preference for } \\
\text { red and blue, but individuals } \\
\text { were extremely flower } \\
\text { constant. }\end{array}$ & Goulson \& Cory (1993) \\
\hline Gonepteryx rhamni & $\begin{array}{l}\text { red/orange/yellow \& } \\
\text { blue/purple }\end{array}$ & Ilse (1928) \\
\hline Vanessa polychloros & red & Ilse (1928) \\
\hline Argynnis paphia & $\begin{array}{l}\text { primary: orange/yellow } \\
\text { secondary: blue/purple }\end{array}$ & Ilse (1928) \\
\hline Macroglossum stellatarum & $\begin{array}{l}\text { primary: } 440 \mathrm{~nm} \\
\text { secondary: } 540 \mathrm{~nm}\end{array}$ & Kelber (1996) \\
\hline Inachis io & yellow \& blue & Ilse (1928) \\
\hline Papilio machaon & blue/purple & Ilse (1928) \\
\hline Papilio demoleus & blue/purple & Ilse \& Vaidya (1956) \\
\hline Papilio troilus & $\begin{array}{l}\text { primary: blue } \\
\text { secondary: orange }\end{array}$ & Swihart (1970) \\
\hline
\end{tabular}

Table 4-1 A summary of the innate flower colour preferences found in Lepidoptera

Butterflies have also been shown to learn to be efficient in their foraging by distinguishing rewarding and non-rewarding flowers, and learning where to probe in a particular species (Kandori \& Ohsaki, 1996; Kandori \& Ohsaki, 1998). They can also learn rapidly to feed from different coloured 
flowers if these are rewarding (Swihart \& Swihart, 1970; Goulson and Cory, 1993; Weiss 1995, 1997; Kandori \& Ohsaki, 1996; Kelber 1996; Lewis \& Lipani, 1990), although increasing the number of species from which they have learnt to feed appears to reduce their efficiency in feeding from the flowers, possibly indicating memory constraints (Lewis, 1986).

It might be expected that butterflies be able to learn which flowers in their particular environment give the best reward, as this may decrease the amount of time they have to spend foraging and therefore increase the amount of time they can spend searching for mates, laying eggs etc. The innate colour preferences may be explained as a way in which the individuals can distinguish flowers for feeding from a predominantly green or spectrally impure background (Klein, 1978; Menzel, 1979) when they first emerge. However, it is not easy to see why different species should have different innate colour preferences when they are not specialised to feed from flowers of a particular colour, so that the nutritional value of the flowers to the butterflies is not related to their colour. This may, therefore, be another case in which butterflies demonstrate a non-functional 'inherent bias' for a particular colour or pattern. In Chapter 2, the flower and mate colour preferences of the polymorphic butterfly Argynnis paphia were investigated, and it was found that the males had a preference for orange females (confirming the work of Magnus, 1958) which was also reflected in their preference for orange flowers (Ilse, 1928). In the case of Argynnis paphia it was not possible to determine whether this preference for orange had evolved for the purpose of mate recognition and simply had the added effect of causing an attraction for orange flowers, or whether the preference was due to an evolutionarily neutral colour bias which was then expressed in both flower and mate choice. Cook et al. (1994) demonstrated a preference in Papilio dardanus for true white morphs (hippocoonides) over yellow morphs (trimeni) over red/orange morphs (lamborni) (see Chapter 3 for an analysis of the colours). Since the white morphs are the most common this could be explained in terms of a rapid learning effect, whereby naïve males mate at random, and then subsequently mate more frequently with females of the same colour. It could also be explained by innate colour preferences, which could be expressed in both mate and flower choice.

\section{$\underline{\text { Aims }}$}

The aims of this chapter are to determine the innate flower colour preferences of Papilio dardanus, and to investigate the degree of constancy it shows to colours, indicating the colour learning capabilities of the species. These can then be compared with the mate choice behaviour studied in Chapter 5 . 


\section{Experiment 4-1 Flower colour preference and constancy in Papilio dardanus}

\section{Introduction}

As summarised in Table 4-1, different species of butterfly have been shown to have different colour preferences in their naïve choice of flower. Crane (1955) described 'tropical Papilionids and Pierids' as feeding predominantly from red flowers at her research station in Trinidad. However, experiments carried out to test the naïve preference of three species of the genus Papilio showed a primary preference for flowers in the blue area of the spectrum (Papilio machaon, 1lse, 1928; Papilio demoleus, Ilse \& Vaidya 1956; Papilio troilus, Swihart, 1970), as did two out of the three experiments carried out on species of the genus Pieris (Pieris brassicae, Scherer \& Kolb, 1987; Pieris rapae, Kandori \& Ohsaki, 1996; equal red/blue preference in Pieris napi, Goulson \& Cory, 1993). This suggests that these butterflies have an initial preference for the blue region of the spectrum which, at least in some geographic regions, may subsequently be altered by learning to allow the butterflies to exploit the most rewarding flowers.

The order of colour preferences found in mate choice in Papilio dardanus by Cook et al. (1994) could be due to an innate bias for certain colours or, since the ranking of morph preferences reflects the proportions of the different morphs, due to a learning effect in males. Some butterflies have been shown to demonstrate a high degree of flower constancy (Goulson \& Cory, 1993; Kandori \& Ohsaki, 1996) - returning again and again to the same colour. This may indicate that they can learn that a certain colour flower is profitable from a single visit, and this type of 'colour imprinting' could explain the mate choice preferences of Papilio dardanus.

\section{Aim}

The aim of this experiment is to ascertain the initial flower colour preference of naïve Papilio dardanus butterflies, and to measure the degree of flower colour constancy shown by individuals in order to assess the rapid colour learning capabilities of the species.

\section{Method and Materials}

Individual Papilio dardanus of either sex which had not fed from flowers before were placed in a flight cage (see Appendix 3 for dimensions and further details) - up to 15 individuals were present in the cage at any one time. In the centre of the cage was placed a $2 \times 3$ array of artificial flowers 
(diameter $5 \mathrm{~cm}$, resembling poppies - see Figure 4-1) each containing sugar water at a concentration of approximately 1 part sugar to three parts water. The flowers were painted red (reflectance $640 \mathrm{~nm}$ and above), blue (reflectance between $400-500 \mathrm{~nm}$ with peak at $460 \mathrm{~nm}$ ) and yellow (reflectance above $580 \mathrm{~nm}$ ) (all enamel paint, Humbrol Ltd., Hull; reflectance spectra from the manufacturer), and placed in an array. None of the paints had any UV reflectance. It was decided not to include a true-white (achromatic) flower, which would represent the most favoured colour in the case of mate preference. This is because discrimination between different hues and different brightnesses of achromatic stimuli are carried out by different neural mechanisms, and butterflies show an almost linear relationship between the brightness and attractiveness of achromatic flower models (Swihart, 1971). Therefore only red and yellow were used in the experiment, and compared with blue, which has been shown to be innately attractive to Papilionids (see Table 4-1). The array was placed across the width of the rectangular cage (East-West), with $12 \mathrm{~cm}$ between adjacent flower centres and adjacent flowers of each of the three pairs being the same colour (See Figure 4-2). Pairs of flowers were used in order to decrease the effect of any individual differences between the flowers which might affect the recorded colour choices of the butterflies.

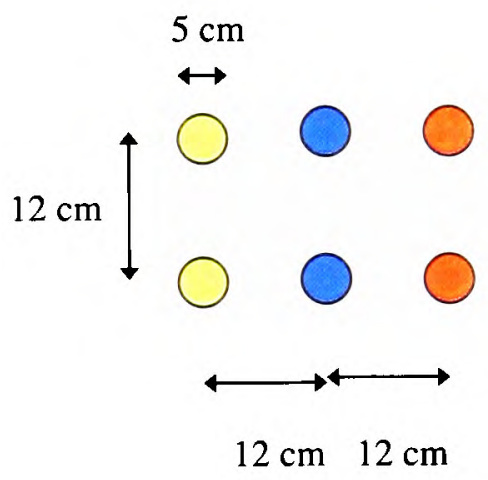

Figure 4-2 The experimental flower array for Experiment 4-1 (position of colours was altered throughout the experiment)

As soon as an individual landed on a particular flower and extended its proboscis, the colour and position (North or South) of the flower on which it landed was recorded. The butterfly was allowed to feed, and the underside of its wings were marked with a permanent marker to allow subsequent visits by the same individual to be recorded. The length of the visit to the flower was also classified as 'brief' (under approximately 3 seconds), 'medium' (approximately 3-10 seconds), or 'long' (over 10 seconds). After five naïve butterflies had been recorded as making an initial colour choice, the order of the colours was changed to average out any spatial effects, and this was repeated after every batch of five initial choices. Subsequent visits by individuals were also recorded to allow an assessment of colour constancy.

\section{Results}

Butterflies approached the flower array from all directions, but mainly from either the North or South as they flew up and down the length of the cage. 
First choices of butterflies:

\begin{tabular}{|l|c|c|c|}
\hline & South & North & Total \\
\hline Blue & 16 & 9 & $\mathbf{2 5}$ \\
\hline Red & 0 & 1 & $\mathbf{1}$ \\
\hline Yellow & 3 & 1 & $\mathbf{4}$ \\
\hline Total & $\mathbf{1 9}$ & $\mathbf{1 1}$ & $\mathbf{3 0}$ \\
\hline
\end{tabular}

Table 4-2 The initial flower choices of naive Papilio dardanus
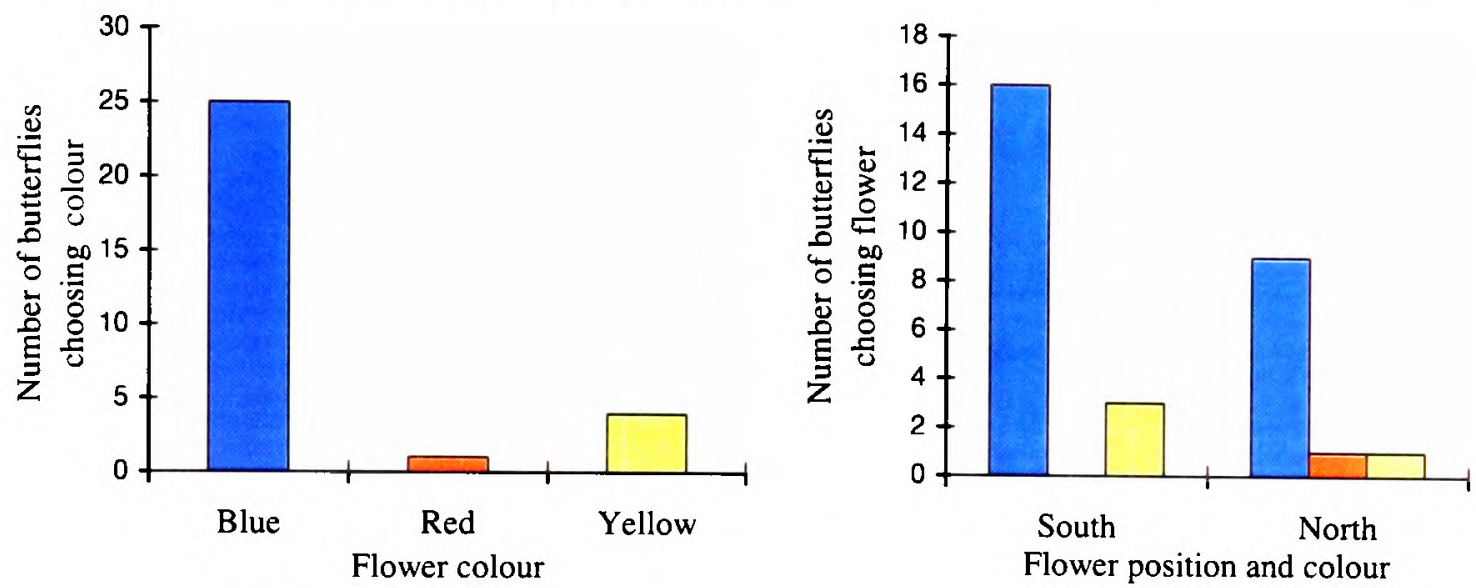

Figure 4-3 The distribution of the initial flower choices of Papilio dardanus

This data indicates that there is no significant preference for flowers at the North or South of the array ( $p=0.100$, binomial test, $n=30$ ), but that there is a significant preference for blue over red and yellow flowers $\left(\mathrm{p}<4 \times 10^{-8}, \chi^{2}=34.2, \mathrm{n}=30\right)$.

All recorded flower visits:

\begin{tabular}{|l|c|c|c|}
\hline & South & North & Total \\
\hline Blue & 117 & 110 & $\mathbf{2 2 7}$ \\
\hline Red & 4 & 1 & $\mathbf{5}$ \\
\hline Yellow & 28 & 25 & $\mathbf{5 3}$ \\
\hline Total & $\mathbf{1 4 9}$ & $\mathbf{1 3 6}$ & $\mathbf{2 8 5}$ \\
\hline
\end{tabular}

Table 4-3 The total recorded flower choices by Papilio dardanus

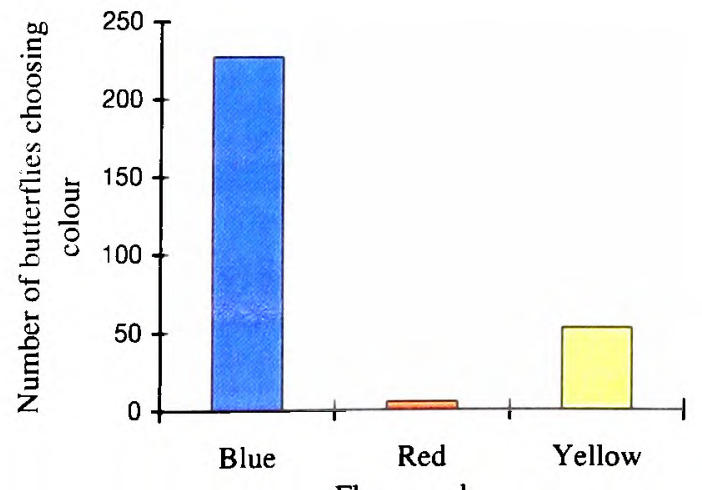

Flower colour

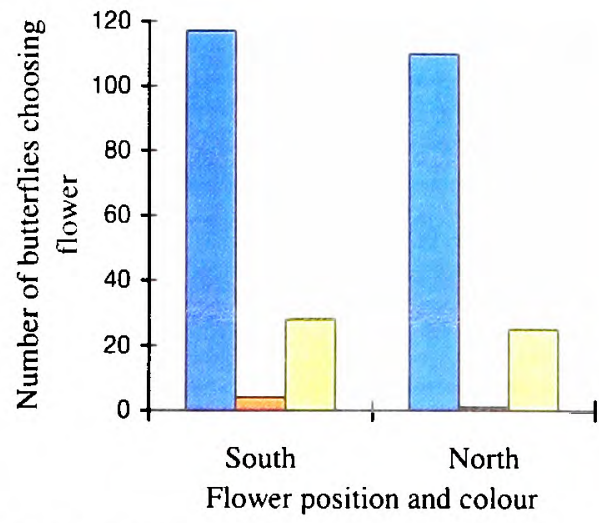

Figure 4-4 Distribution of all the recorded flower choices by Papilio dardanus 
The data for all recorded flower choices shows a distribution which is not significantly different from this $(\mathrm{p}=0.135, \mathrm{n}=285$, Friedman Test).

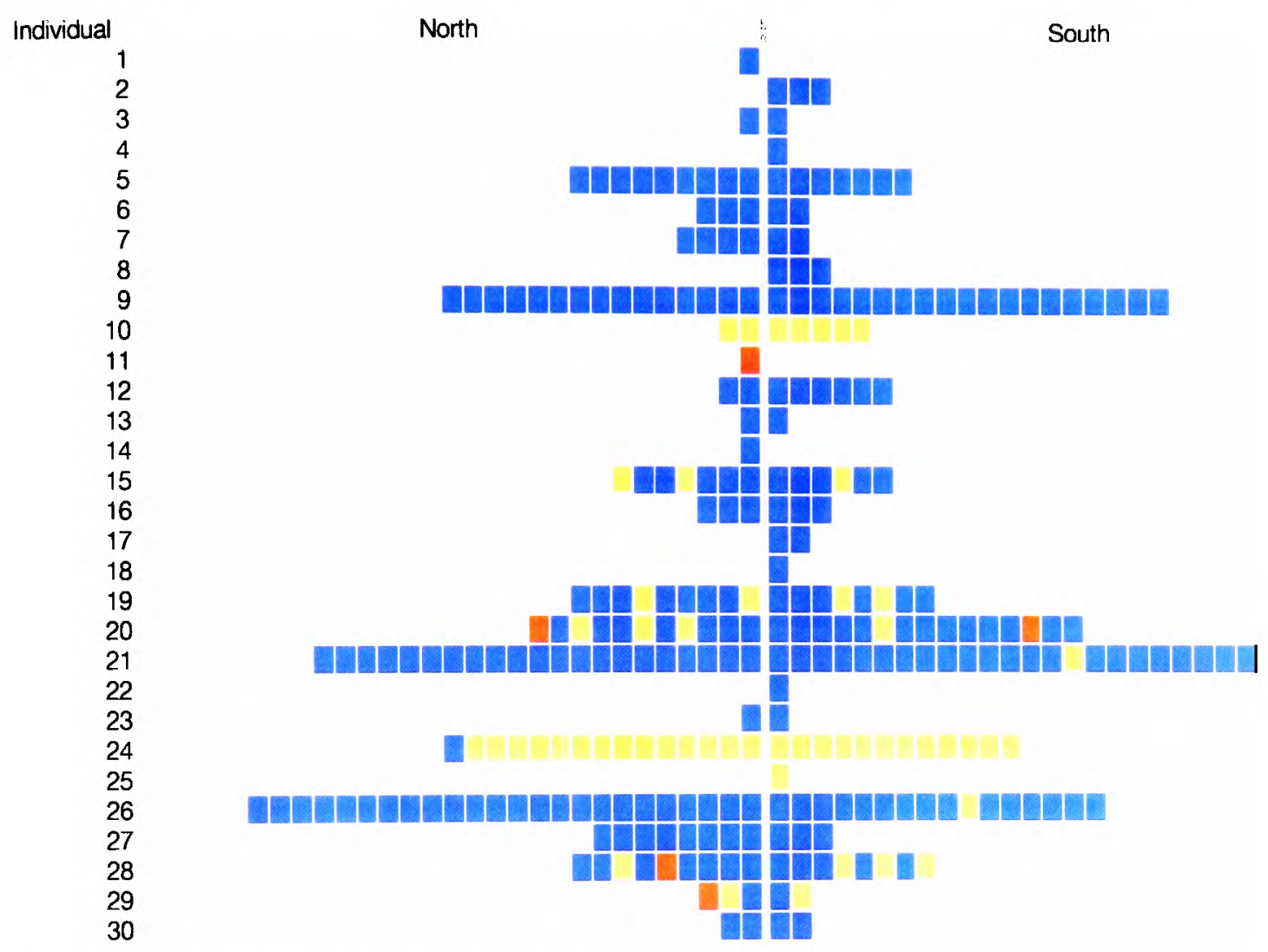

Figure 4-5 Plot of all recorded flower visits by individual Papilio dardanus

A constancy index was used, where:

constancy to first choice of flower $=$ no. visits to same colour as $1^{\text {si }}$ choicel total no. visits

and:

constancy to favourite colour $=$ no. visits to most visited colour/ total no. visits

These were calculated for each butterfly which had visited the flowers more than once and a mean value for each obtained:

constancy to $1^{\text {st }}$ choice $=0.90$ (standard deviation $0.21, \mathrm{n}=30$ )

constancy to favourite colour $=0.927$ (standard deviation $0.149, \mathrm{n}=30$ )

These figures are very similar, as only one butterfly (no. 19, see Figure 4-5) actually visited a flower first which was not subsequently visited the most often. In addition, only two individuals (nos. $10 \& 24)$ had favourite colours which were not blue, but it is interesting that both of these showed 
very high constancy to this colour (100\% in the case of butterfly 10 , and $96 \%$ in the case of butterfly 24). This suggests that the high degree of constancy shown may be due to learning rather than innate preference (unless there is a degree of polymorphism in the innate preference).

Since butterflies were often observed to alternate between the North and South flower of the same colour, the number of visits to the same and the alternative position (either 'North' or 'South' regardless of colour) were counted:

\begin{tabular}{|c|c|}
\hline Number of visits to the same position & Number of visits to the alternative position \\
\hline 83 & 162 \\
\hline
\end{tabular}

Table 4-4 The number of visits of Papilio dardanus to flowers in different spatial positions during feeding

This shows that the butterflies are visiting the flower at the alternative position on the array (despite their high colour constancy) significantly more often than returning to the same position $\left(\mathrm{p}=1.3 \times 10^{-7}\right.$, binomial test, $\mathrm{n}=245$ ).

\section{Discussion}

This experiment demonstrates that Papilio dardanus show a significant preference for blue flowers over yellow and red. They are not significantly changing their behaviour on subsequent visits, and show no significant spatial preferences within the array. They show a high degree of constancy (90\%) to a colour when the flowers provide a reward, and this colour is almost always the first to which the butterfly has made a visit - only one individual had a favourite colour which was not its first choice. It therefore appears that the butterflies are learning a profitable flower colour from their first visit, and subsequently visit the same colour $90 \%$ of the time.

It is interesting to plot a graph to show the length of the feed (classified as 'brief', 'medium' or 'long'), and to compare the overall proportions of the three feeding lengths (representing 'normal feeding') compared with the categorised length of the feed just before a butterfly chooses to feed off a new colour:

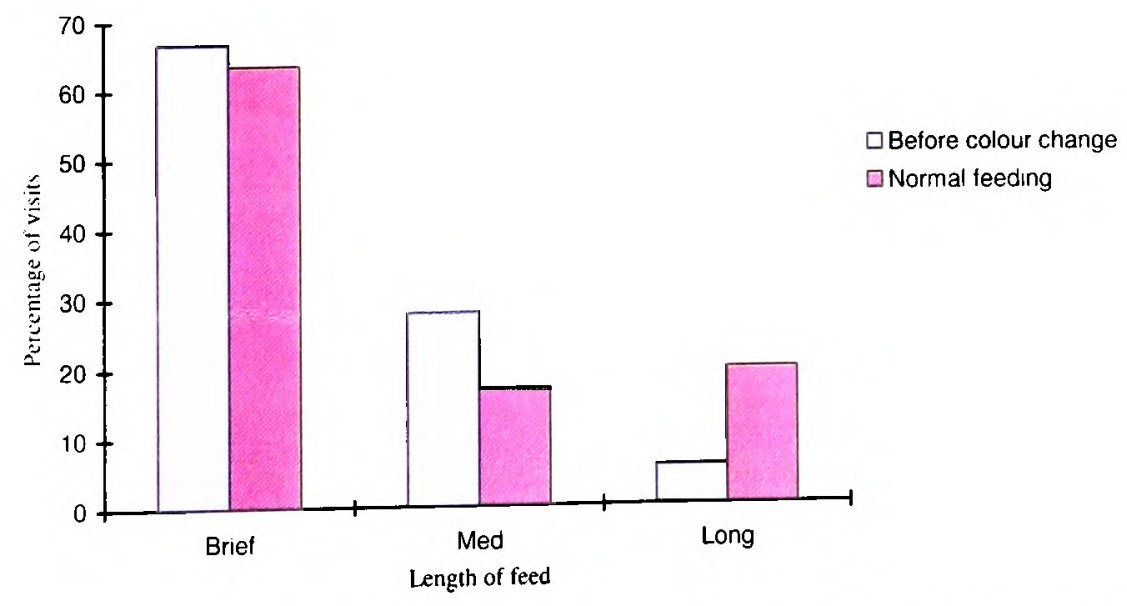

Figure 4-6 Length of visit to flowers by Papilio dardanus during normal feeding, and just before a colour change was recorded 
These data suggest that the butterflies tend to try a new colour when they have failed to feed successfully (indicated by a 'brief' visit) from their initial colour choice. This is backed up by the evidence from one butterfly (who up until that point had fed exclusively off yellow flowers) to an array of empty flowers. This data was gathered when the array was introduced to the flight cage full of butterflies at the start of an experimental session. The butterfly came directly down to the array before the flowers had been filled up with sugar water. Her choices in order were:

\begin{tabular}{|c|c|c|c|c|c|}
\hline \multicolumn{2}{|c|}{ Blue } & \multicolumn{2}{|c|}{ Yellow } & \multicolumn{2}{c|}{ Red } \\
South & North & South & North & South & North \\
\hline & I & & & & \\
\hline & & & 2 & & \\
\hline & & 3 & & & \\
\hline 4 & & & & & \\
\hline & 5 & & & & \\
\hline & & 6 & & & \\
\hline 8 & & & & 7 & \\
\hline
\end{tabular}

Table 4-5 The flower visits made by an individual Papilio dardanus when the flowers contained no sugar water

In this sequence the butterfly showed highly uncharacteristic colour swapping (and it is unusual that she starts the searching with a blue flower) - she had never been to a flower other than yellow, and when the flowers were filled with sugar water she fed almost exclusively off yellow once again (see individual 24 in Figure 4-5). This supports the idea that the butterflies are learning rapidly, from their first profitable feed, and will stick to the first colour from which they feed successfully, only trying other colours when they fail to find food from their original colour choice.

The butterflies also show a significant alternating behaviour between the pairs of flowers of the same colour (see Table 4-4). This presumably increases their chances of finding nectar in a natural situation, as they may have emptied one flower on their last visit and it may need time to refill. In addition, it was also noted that the butterflies appeared to try more colours when there were more individuals feeding - several of the cases of a butterfly choosing a colour other than its usual favourite occurred when other individuals were feeding at the array - and this is investigated in later experiments. 


\section{Experiment 4-2 Investigation of the effect of painted vs unpainted flowers on preference}

\section{Introduction}

In Experiment 4-1, all the flowers had been painted with the same enamel paint (from Humbrol Ltd, Hull). To investigate whether or not the glossy painted surface was more or less attractive to the naïve butterflies than a more natural, matt, surface, a pilot experiment was designed using unpainted artificial flowers, since even matt paint tended to give a gloss finish when used on the woven plastic surface of the artificial flowers. The artificial poppies used in Experiment 4-1 were originally red in colour, before being painted, and had a naturally matt texture. The original red colour was likely, however, to have a slightly different spectrum from the red of the enamel paint, and comparing the two directly in a choice test might cause the butterflies to distinguish between the two colours rather than show a preference for the texture. Since red was significantly less preferred than blue and yellow in Experiment 4-1, it was decided to use the same artificial flowers as before, but to leave one pair unpainted red in order to compare the number of visits to the unpainted red flowers with the number in the previous experiment to the painted red flowers. It was hoped that this method might be more successful at detecting a texture preference than a direct comparison between unpainted and painted red flowers.

\section{Aim}

The aim of this experiment is to determine whether or not naïve Papilio dardanus show a significant preference for red flowers over blue and yellow when the red flowers are left unpainted, with a matt texture, whilst the blue and yellow flowers have a gloss paint finish.

\section{Method \& Materials}

Individual Papilio dardanus of either sex which had not fed from flowers before were placed in the flight cage, with up to 20 individuals in the flight cage at any one time. In the centre of the cage was placed a $2 \times 3$ array of artificial flowers (diameter $5 \mathrm{~cm}$, resembling poppies), as in Figure 4-2. Two artificial flowers were left unpainted, with a matt red surface, and the others were painted blue and yellow (enamel paint, Humbrol Ltd., Hull), and placed in the array, with $12 \mathrm{~cm}$ between adjacent flower centres, adjacent flowers of each of the three pairs being the same colour, as in Experiment 4-1 (see Figure 4-2). As before, pairs of flowers were used to decrease any effects introduced by individual differences between the flowers. Since in this experiment only the initial flower choices of the naive butterflies was being recorded, the flowers were left empty, with no sugar water in them. As soon as an individual landed on and tried to feed from a particular flower, it was removed from the 
cage, and the choice recorded to determine the initial preferences of the butterflies. After every ten data points were recorded, the order of the colours was changed to average out any spatial effects from the positions of the flowers.

\section{Results}

\begin{tabular}{|c|c|c|}
\hline Number choosing blue (gloss) & Number choosing yellow (gloss) & Number choosing red (matt) \\
\hline 1 & 2 & 27 \\
\hline
\end{tabular}

Table 4-6 The texture and colour preferences of naïve Papilio dardanus

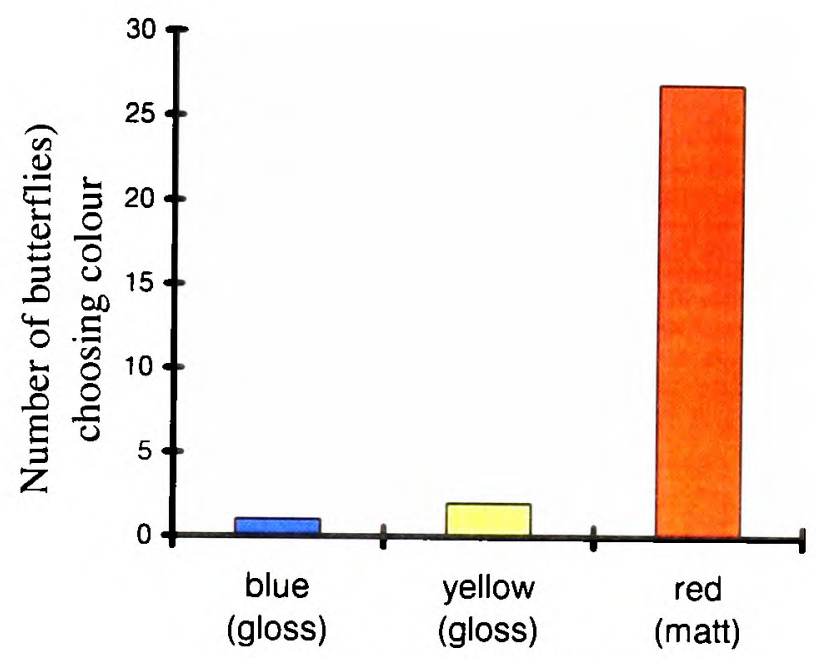

Flower colour/texture
The results indicate that the Papilio dardanus now have a strongly significant preference for the matt red flowers over the gloss blue and yellow $\left(\mathrm{p}<4 \times 10^{-10}, \chi^{2}=\right.$ $43.4, n=30$ ). This is a very big shift in behaviour when compared with the results of Experiment 4-1 where naïve butterflies showed a significant preference for blue flowers when all were painted with colours having the same gloss finish.

Figure 4-7 Flower texture preference in Papilio dardanus.

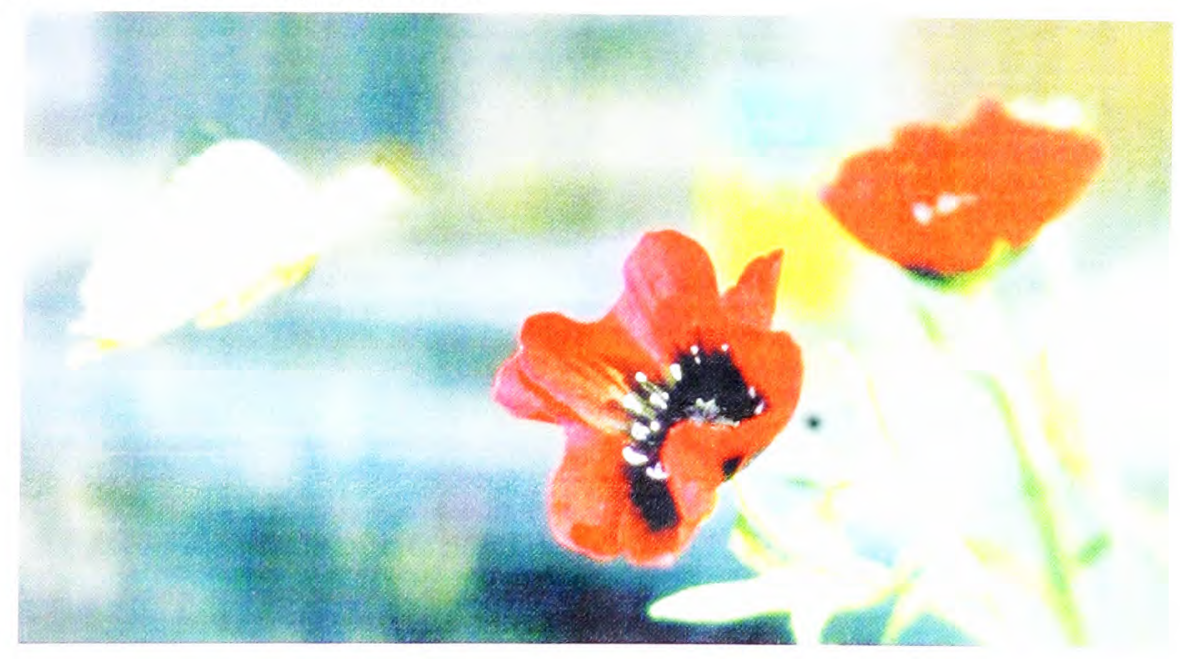

Figure 4-8 A male Papilio dardanus approaching a red unpainted flower of the type used in Experiment 4-2

\section{Discussion}

The results of this experiment indicate that the butterflies are not choosing between the flowers simply on the basis of their colours, and that there is another factor determining their choice 
which appears to be more important to them and can shift their colour preferences dramatically. Unfortunately, there were differences between the painted and unpainted red flowers other than texture in this pilot experiment, such as the darkness of the central area (see Figure 4-8), which had been painted over in Experiment 4-1. A further experiment was therefore planned to investigate the differences between the unpainted red flowers and the painted red flowers which might be affecting the choices of the butterflies.

\section{Experiment 4-3 The effect of matt/gloss finish and colour of flower centre on preference}

\section{Introduction}

The previous experiments showed that the butterflies significantly preferred unpainted red flowers over painted blue and yellow flowers, but significantly preferred blue flowers over red and yellow when all three colours were painted using the same gloss enamel paint. Therefore the choices of the butterflies appear to be influenced by a cue other than colour. Two differences between the painted and unpainted flowers were immediately obvious - firstly the unpainted flowers were made of a plastic fabric which had a matt finish rather than the uniform gloss finish of the painted flowers, and secondly they had black central markings, which could act as honey guides, which had been painted over in the painted flowers. It seemed likely that either of these two factors could be important (Kandori \& Ohsaki have demonstrated the importance of honey guides, 1998), and therefore an experiment was designed to test whether either of these could be influencing the butterflies' choices.

\section{Aim}

The aim of this experiment is to ascertain whether or not the butterflies show a preference for matt flowers over gloss flowers, and whether or not they are attracted to flowers with a dark central area over those without one.

\section{Method \& Materials}

Naïve Papilio dardanus of either sex were placed in the flight cage, with up to 20 individuals in the cage at any one time. In the centre of the cage, as in Experiments 4-1 and 4-2, was placed an array of the same artificial flowers. The array in this experiment was made up of only four flowers, although the distances between the centres of the flowers were still $12 \mathrm{~cm}$ (see Figure 4-9) - two were painted red (using the Humbrol enamel paint), but on one of these black central markings were made with a permanent marker pen similar to the markings on the unpainted red flowers. Two were painted blue - one gloss as before, but the other was made matt by covering the petals with cloth and painting the cloth with the same enamel paint (from Humbrol). This gave a matt finish to the flower. 


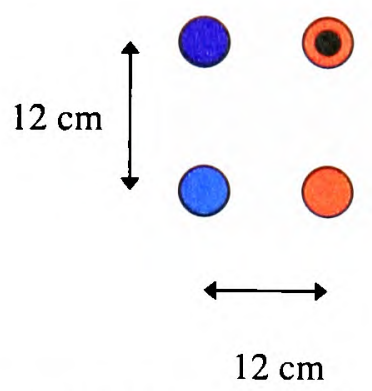

Figure 4-9 The experimental array of flowers used in Experiment 4-3, where dark blue represents a matt blue finish of the same colour as the gloss flower below it

The flowers were filled with sugar water (approximately three parts water to one part sugar), and naïve Papilio dardanus were allowed to feed from them freely as in Experiment 4-1. When a butterfly landed on the array for the first time, its initial choice of flower was recorded, and the underside of its wings was marked with a permanent marker as in Experiment 4-1 without disturbing it from feeding. This allowed the butterflies to be recognised individually, so that subsequent visits to the flowers by the same individuals could be recorded. The flower array was rotated $180^{\circ}$ after 15 data points had been recorded to remove any spatial effects from the results, but the two gloss flowers were always adjacent, as were the matt and the black centred flowers.

\section{Results}

The results of the first choice of butterflies is shown in Table 4-7 and Figure 4-10.

\begin{tabular}{|l|c|c|c|c|}
\hline & Gloss Blue & Matt Blue & Plain Red & Red, Black Centre \\
\hline No. $1^{\text {st }}$ Visits & 17 & 5 & 6 & 1 \\
\hline
\end{tabular}

Table 4-7 The initial flower choices of naïve butterflies in Experiment 4-3

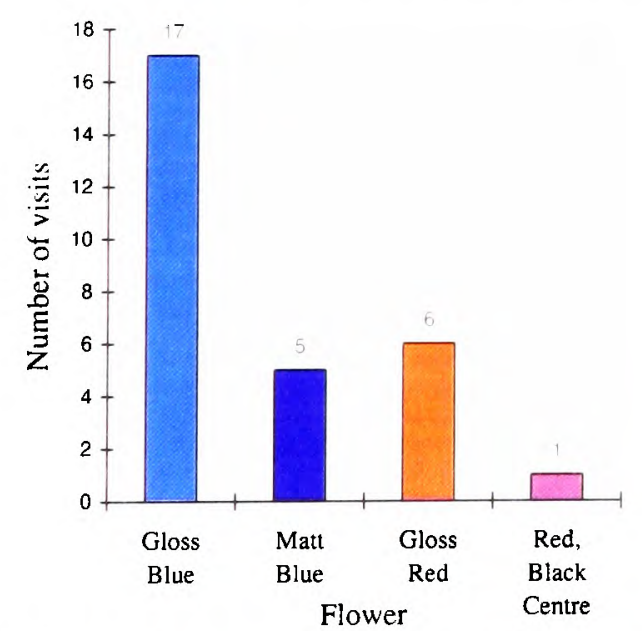

Figure 4-10 Graph showing the number of first visits by Papilio dardanus to different flowers in Experiment 4-3

The distribution of subsequent choices is shown in Figure 4-11: 


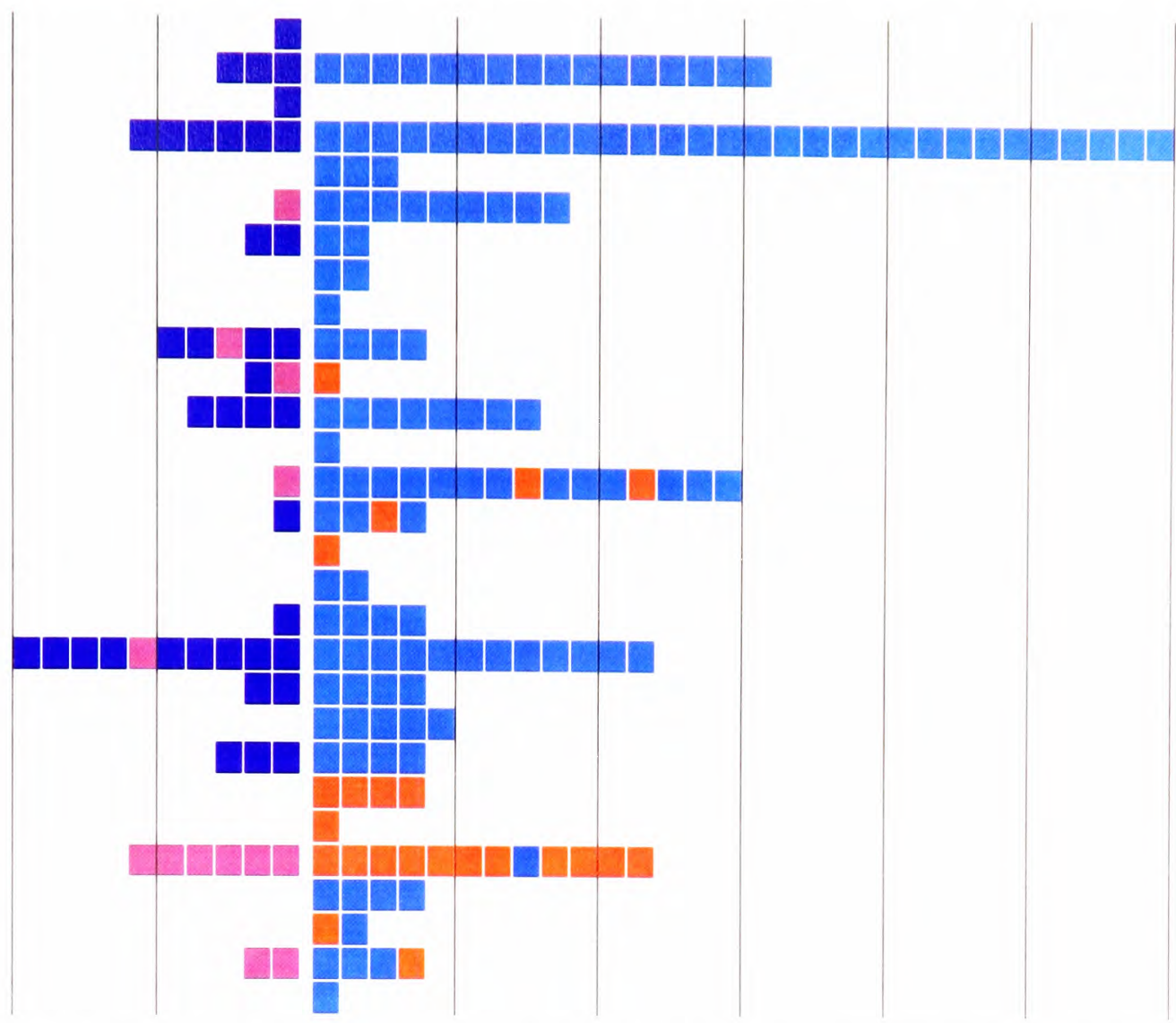

Figure 4-11 Plot of all the recorded flower choices of Papilio dardanus, with bright blue and bright red on the right representing the gloss flowers, the dark blue on the left representing the matt blue, and the dark red on the left representing the gloss red with a black centre.

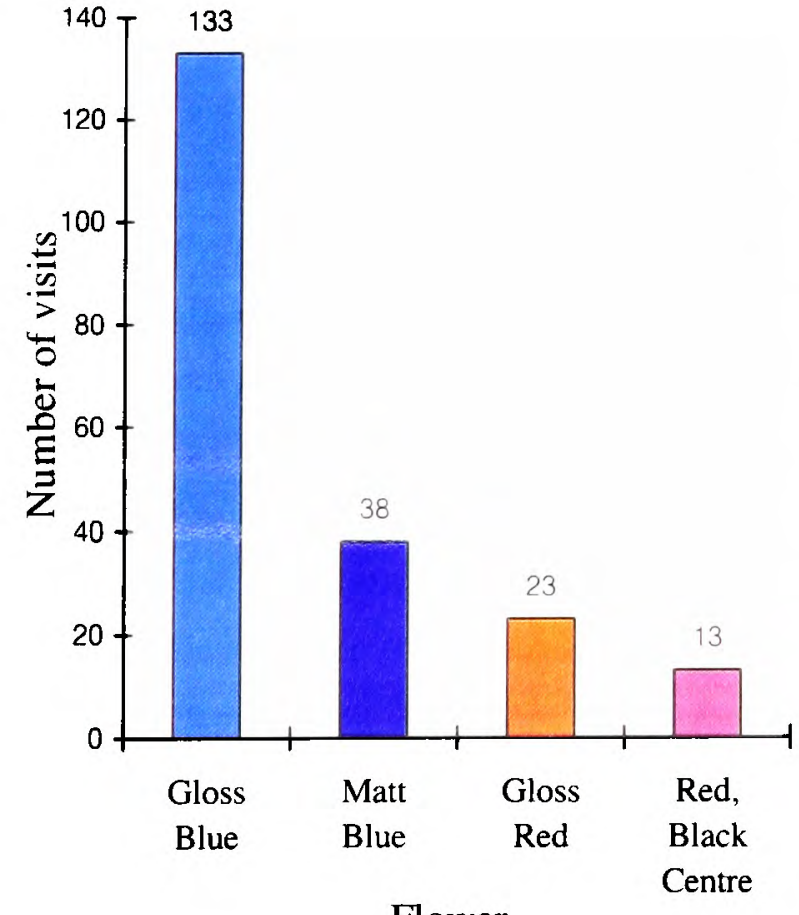

Flower
The results show that the butterflies display a significant preference for gloss blue flowers over any of the other options on their first choice of flower $\left(\mathrm{p}<0.0003, \chi^{2}=19.4\right.$, $\mathrm{n}=29$, ), and an even greater bias for gloss blue on their subsequent choices, although this may well be due to the fact that the fabric of the matt flower soaked up sugar water and thus the flower was less rewarding. There is no significant difference between the overall choices of red flowers and red flowers with a black centre ( $\mathrm{p}=0.066$, binomial test, $\mathrm{n}=36$ ). Calculations of the butterflies' constancy to a particular flower can be calculated as in Experiment 4-1: 
Constancy to $1^{\text {st }}$ flower chosen $=0.660($ standard deviation $=0.28, \mathrm{n}=29)$

Constancy to favourite flower $=0.733$ (standard deviation $=0.22, \mathrm{n}=29$ )

These figures are lower than the constancies recorded in Experiment 4-1, but this is probably due to some individuals failing to distinguish between the matt and gloss blue flowers, and between the red flower and red flower with a black centre. If the choice is reduced to simply red or blue, the constancies recorded are:

Constancy to $1^{\text {st }}$ flower chosen $=0.871$ (standard deviation $=0.234, \mathrm{n}=29$ )

Constancy to favourite flower $=0.908($ standard deviation $=0.158, n=29)$

These are close to the values of 0.90 and 0.927 recorded in Experiment 4-1.

\section{Discussion}

These results suggest that neither the matt texture nor the presence of black central markings in the flowers are significantly attractive to Papilio dardanus. In fact the gloss finish was significantly preferred to the matt finish. The butterflies remain highly constant to a particular flower, but many appear not to discriminate between the matt and gloss and the red flower and red flower with black centre, simply remaining $90 \%$ constant to a particular colour. It therefore appears that the butterflies are preferring a gloss finish to the flowers, and that they do not significantly prefer the red flowers with the black centre to the plain red flowers (in fact they show an insignificant preference for the flower without the black centre). The chemical constituents of the paint itself would not seem to be particularly repellent, as the blue-painted flowers used in Experiment 4-1 were the most freshly painted (only hours before the first experimental session), and were strongly preferred over the red and yellow flowers which had been painted some months previously. Many of the chemicals present in the flowers would also not be detectable to the butterflies until they had landed, when tarsal receptors may be used. It is possible that there is some feature of the artificially matt flower in Experiment 4-3 which is unattractive to them and therefore biasing the results. Unfortunately further experiments to investigate this phenomenon were not possible due to time constraints, and the fact that further artificial flowers of the same design were unobtainable. The preference for the unpainted red flowers in Experiment 4-2 therefore remains unexplained. 


\section{Experiment 4-4 The effect of another individual feeding on choice of colour}

\section{Introduction}

Whilst Experiments 4-1 and 4-3 were being carried out, it was noticed that the Papilio dardanus tended to feed more often, and from a wider range of colours when other individuals were feeding from the array. In fact some of the instances where an individual fed off a flower which was not its 'favourite' colour in Experiment 4-1 coincided with other butterflies being present on the array (e.g. individual 29's final three visits in Figure 4-5). It thus seemed that although Papilio dardanus had an initial preference for blue, they could also learn from other individuals to feed from other colours. This is another aspect of feeding behaviour which appears not to have been investigated before, and could be a very interesting way for naïve butterflies to increase their feeding efficiency rapidly.

An experiment was therefore planned to test whether the presence of another individual feeding on the array would influence the colour choices of other individuals. In order to simulate the presence of an individual feeding at the array, a dead butterfly (with its wings open) was used, pinned on to one of the flowers. This allowed complete control of the appearance of the 'teacher' individual.

\section{Aim}

This experiment aims to determine whether the apparent presence of another individual feeding affects the colour choice of naïve Papilio dardanus.

\section{Method \& Materials}

Since an experiment had already been carried out on the initial colour preferences of Papilio dardanus, without the influence of other individuals feeding (Experiment 4-2, in which individuals were removed as soon as they had alighted on a flower in order to feed), the results of Experiment 4-2 could be used as the control for an experiment on the influence of another individual at the array. Therefore, the same array as had been used in Experiment 4-2 (with unpainted red flowers versus painted blue and yellow flowers) was used in this experiment.

Naïve Papilio dardanus of either sex were placed in the flight cage, with up to 20 individuals in the cage at one time. The array of flowers used in Experiment 4-2 (see Figure 4-2) was placed in the centre of the cage as before. A dead male Papilio dardanus, with wings open, was pinned onto a flower of each colour in turn (being moved after every 5 data points was recorded) so as to be obvious from every approach angle but not obscuring the flower. When a butterfly landed on a flower and 
extended its proboscis, the flower colour and position was recorded, as was the sex of the butterfly concerned. It was then removed immediately so as not to influence the choice of any of the other butterflies in the cage.

\section{Results}

With a male pinned onto a blue flower (order blue, yellow, red):

\begin{tabular}{|c|c|c|}
\hline Number choosing blue & Number choosing yellow & Number choosing red \\
\hline 2 & 1 & 7 \\
\hline
\end{tabular}

Table 4-8 Visits of Papilio dardanus to flowers with a dead male pinned onto blue flower

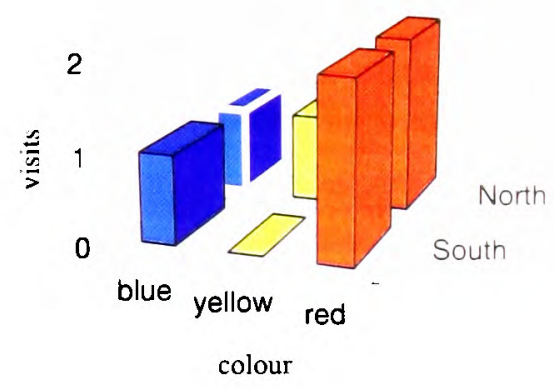

b)

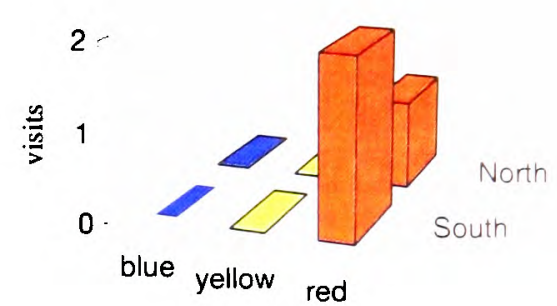

colour

Figure 4-13 Visits of Papilio dardanus to flowers with a dead male pinned onto a) North blue flower; b) South blue flower.

With a male pinned onto a yellow flower (order blue, yellow, red):

\begin{tabular}{|c|c|c|}
\hline Number choosing blue & Number choosing yellow & Number choosing red \\
\hline 4 & 0 & 6 \\
\hline
\end{tabular}

Table 4-9 Visits of Papilio dardanus to flowers with a dead male pinned onto yellow flower

a)

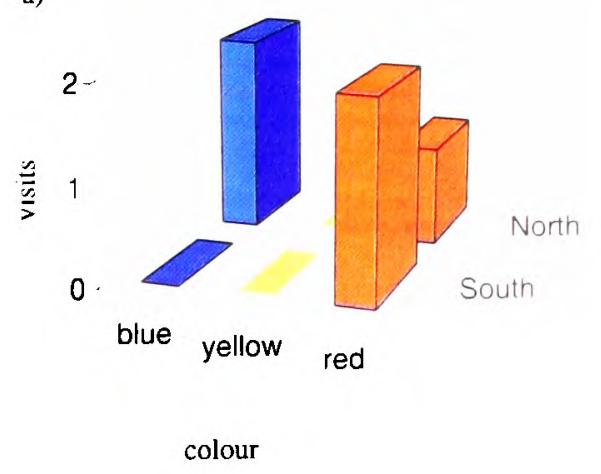

b)

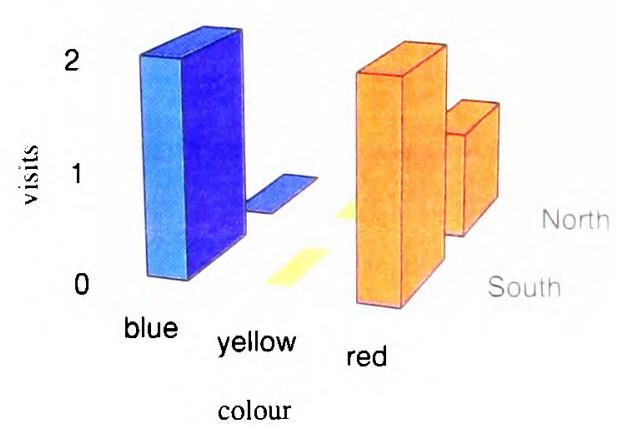

Figure 4-14 Visits of Papilio dardanus to flowers with a dead male pinned onto a) North yellow flower; b) South yellow flower. 
With a male pinned onto a red flower (order blue, yellow, red):

\begin{tabular}{|c|c|c|}
\hline Number choosing blue & Number choosing yellow & Number choosing red \\
\hline 3 & 2 & 5 \\
\hline
\end{tabular}

Table 4-10 Visits of Papilio dardanus to flowers with a dead male pinned onto red flower

a)

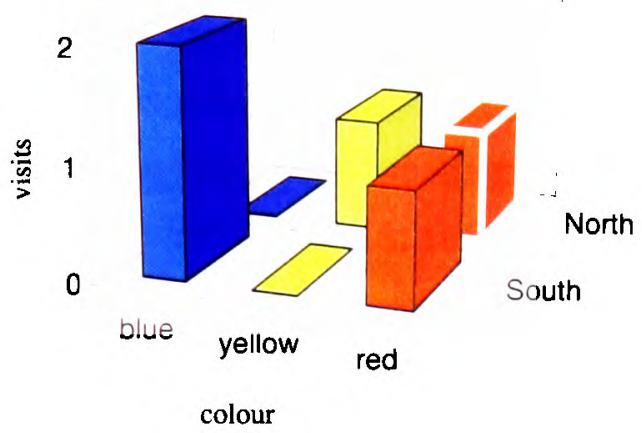

b)

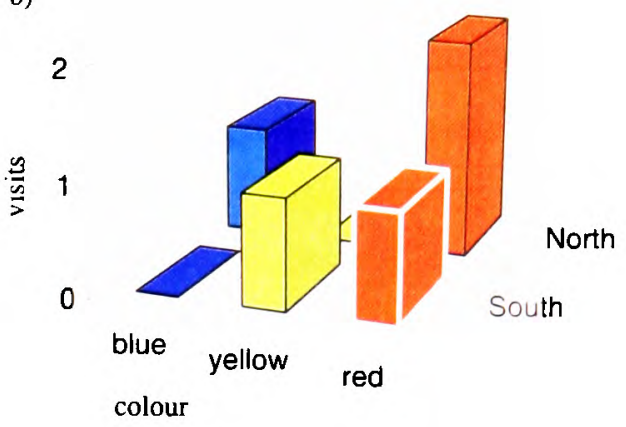

Figure 4-15 Visits of Papilio dardanus to flowers with a dead male pinned onto a) North red flower; b) South red flower.

Taking the data as a whole, the totals are:

\begin{tabular}{|c|c|c|}
\hline Number choosing blue & Number choosing yellow & Number choosing red \\
\hline 9 & 3 & 18 \\
\hline
\end{tabular}

Tabie 4-11 Visits of Papilio dardanus to flowers with another individual feeding

Comparing the results of this experiment, shown in Table 4-11, with those of Experiment 4-2, shown in Table 4-6, indicates that the pinning of a dead male onto various flowers has made a significant difference to the initial choices of the butterflies (using Table 4-6 to generate the probabilities of choosing red or other colour, and then using these to perform a binomial test on the results in Table 4-11: $\mathrm{p}=0.000015, \mathrm{n}=30$ ). The yellow and blue flowers are now visited significantly more often.

Splitting the colour choices into those gathered from males and those from females, the results were:

\begin{tabular}{|l|c|c|c|}
\hline & Number choosing blue & Number choosing yellow & Number choosing red \\
\hline Males & 7 & 2 & 11 \\
\hline Females & 2 & 1 & 7 \\
\hline
\end{tabular}

Table 4-12 The colour preferences of Papilio dardanus of either sex in Experiment 4-4

This data set is difficult to analyse statistically (as the numbers of visits to yellow and blue flowers are so small), but it is possible to see that the two sexes rank the colours in the same order, and appear to show very similar behaviour. 


\section{Discussion}

Although the experiment shows that pinning a dead butterfly onto a flower does alter the choice of other butterflies, they do not simply seem to choose flowers of the same colour as that onto which the dead butterfly is pinned. In fact, when the butterfly is pinned on a blue flower (see Table 48 ), only two individuals chose blue (as opposed to four and three when the butterfly was pinned onto yellow and red respectively - see Table 4-9 and Table 4-10). When the butterfly was pinned on a yellow flower (see Table 4-9), yellow was never chosen (as it was once and twice when the butterfly was pinned onto blue and red respectively - see Table 4-8 and Table 4-10). When the butterfly was pinned on a red flower (see Table 4-10), red was chosen only five times (rather than seven or six times when the butterfly was pinned onto blue and yellow respectively - see Table 4-8 and Table 4-9). The possibility that the butterflies are avoiding the flowers on which the dead one is pinned is ruled out by the fact that out of the seven occasions when the butterflies went to the same colour flower as the one on which a butterfly was pinned, three were visits to the same actual flower as the one on which the individual was pinned, and four were to the other one of the same colour.

On the basis of the data shown in Figure 4-13, Figure 4-14, and Figure 4-15 it is possible that the butterflies are choosing a neighbouring flower to the one on which an individual appears to be feeding. When the butterfly was pinned onto the North blue flower, this effect is clear (although the preference for red is still evident) - when the butterfly was pinned onto the South blue there is not enough data to show any effect. When the butterfly was pinned onto a yellow flower, the adjacent blue flower appears to get more visits, although there is oddly no visit at all to either yellow flower. When the butterfly was pinned on a red flower, the effect on the visits to the adjacent yellow flower seems to follow the same pattern. However, the data sets are very small as this experiment was designed to test for a preference in colour rather than spatial position, and so another experiment was designed in order to test the new hypothesis that the butterflies are attracted to a flower which is spatially close to the one on which another individual appears to be feeding.

\section{Experiment 4-5 Effect of another individual on flower choice}

\section{Introduction}

Experiment 4-4 indicated that the presence of another individual (in the form of a dead male pinned onto a flower) affected the flower choice of other Papilio dardanus. The butterflies did not appear to be choosing flowers of the same colour as the one on which the male was pinned, but there 
was some evidence that they were choosing flowers which were spatially close to that on to which another individual had apparently landed.

'Imitation' has been reported in many vertebrates, but the terminology has been very confused (for reviews, see Galef, 1988 and Whiten \& Ham, 1992). Many terms have been used for the type of behaviour which appears to be present in Papilio dardanus. Galef (1988) suggests 'social enhancement' to refer to the "simple presence of others, presence of behaving others, or presence of residual cues emitted by others .... [which] enhance performance of responses already in an individual's repertoire" (p.13). Within this umbrella term, the butterflies would be showing 'local enhancement' as defined by Thorpe (1963): "apparent imitation resulting from directing the animal's attention to a particular object or to a particular part of the environment" (p.134), or Miller \& Dollard's (1941) 'matched dependent behaviour' in which individuals match their own behaviour to that of other individuals. As Galef (p.19) says, in behaviour of this type, "once the leader (the discriminatory stimulus) departs, those aspects of the follower's behaviour dependent on the presence of the leader are lost. For the pattern of behaviour initiated by the leader to become part of the behavioural repertoire of the follower, independent of the leader, the pattern of behaviour must come under the control of stimuli not dependent on the presence of the leader." So, in this case, by following the feeding behaviour of another butterfly and being rewarded, an individual could increase its repertoire of feeding behaviour. From Experiment 4-4 it appears that this is likely to be through spatial rather than colour learning. This experiment is designed to test this, by removing the colour variation between the flowers and by increasing the size of the array to allow greater sensitivity to spatial positioning.

\section{Aim}

The aim of this experiment is to determine whether or not Papilio dardanus choose flowers spatially close to those from which another individual appears to be feeding.

\section{Method and Materials}

As was mentioned in the discussion to Experiment 4-3, the artificial flowers used in Experiments 4-1, 4-2, 4-3, and 4-4 became no longer available. Therefore, to produce a suitably large array of identical flowers, another design of artificial flower had to be used. Those which were most similar to (but slightly larger than) the artificial poppies used previously were chosen. The flowers were also placed closer together in order to fit a linear array across the width of the flight cage.

Naïve butterflies were introduced to the flight cage (with up to twenty individuals present at one time) in the centre of which was an array of nine red artificial flowers resembling poppies, with an 
average diameter of $8 \mathrm{~cm}$, and with an average separation of $9.5 \mathrm{~cm}$ from the centre of one to the centre of the next in line. The array consisted of nine flowers in a row (see Figure 4-16)

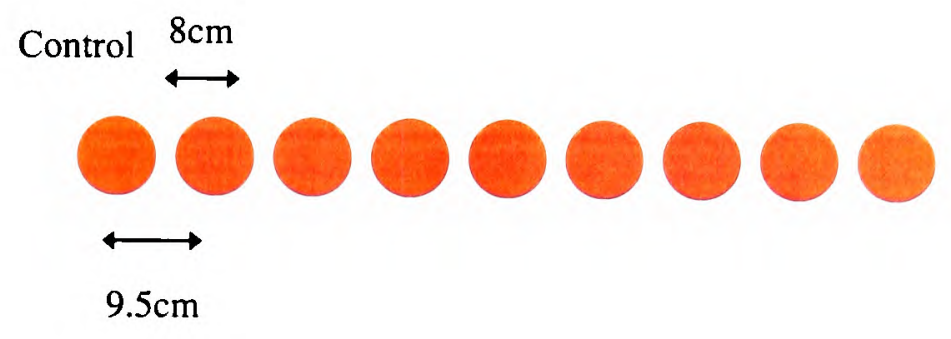

Experiment
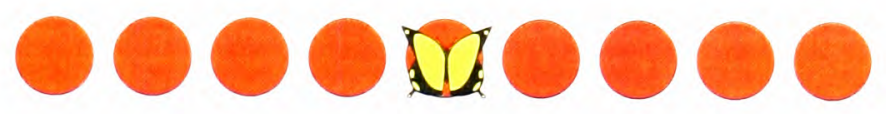

Figure 4-16 The control and experimental arrays used in Experiment 4-5 (the black lines represent the sides of the flight cage)

Since only the initial choice of the butterflies was being recorded, none of the flowers contained sugar water. In the control experiment, the naïve butterflies were allowed to visit the flowers, and as soon as an individual landed on a flower and extended its proboscis, the butterfly was removed, and the position of the flower on which it landed was recorded. For the experimental condition, a dead male Papilio dardanus was pinned onto the central flower, making sure that it was obvious from all angles, and that it did not obscure the flower onto which it was pinned. Visits by naïve butterflies were recorded as in the control experiment, and each butterfly was immediately removed as soon as it had landed on a flower and extended its proboscis so as not to affect the choice of other butterflies. After 15 visits had been recorded under each condition, the flower array was rotated by $180^{\circ}$ (so that the flower which had been number 1 as labelled from west to east became number 9).

\section{Results}

\begin{tabular}{|l|l|l|l|l|l|l|l|l|l|}
\hline Flower & $\mathbf{1}$ & $\mathbf{2}$ & $\mathbf{3}$ & $\mathbf{4}$ & $\mathbf{5}$ & $\mathbf{6}$ & $\mathbf{7}$ & $\mathbf{8}$ & $\mathbf{9}$ \\
\hline Control & 2 & 5 & 3 & 5 & 2 & 7 & 2 & 1 & 3 \\
\hline Exptl. & 2 & 2 & 4 & 3 & 11 & 3 & 2 & 1 & 2 \\
\hline
\end{tabular}

Table 4-13 Visits of Papilio dardanus to a linear array of flowers with or without another individual present on flower 5 
a)

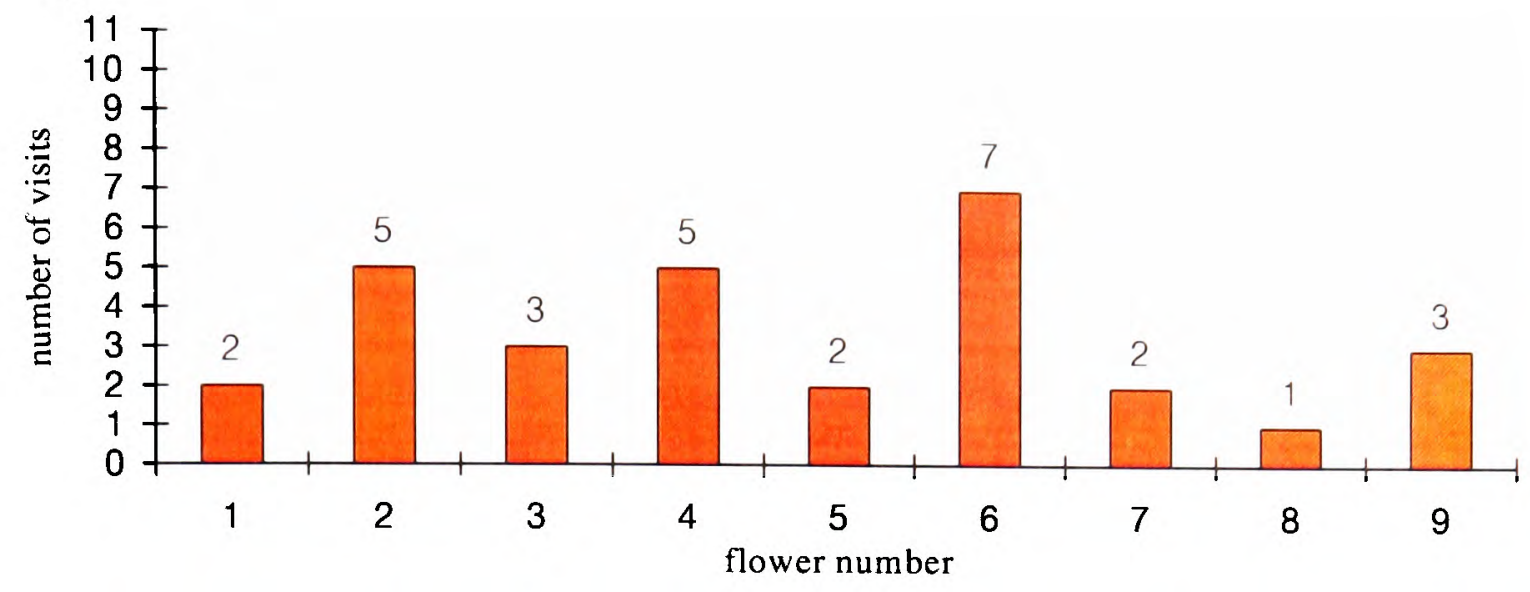

b)

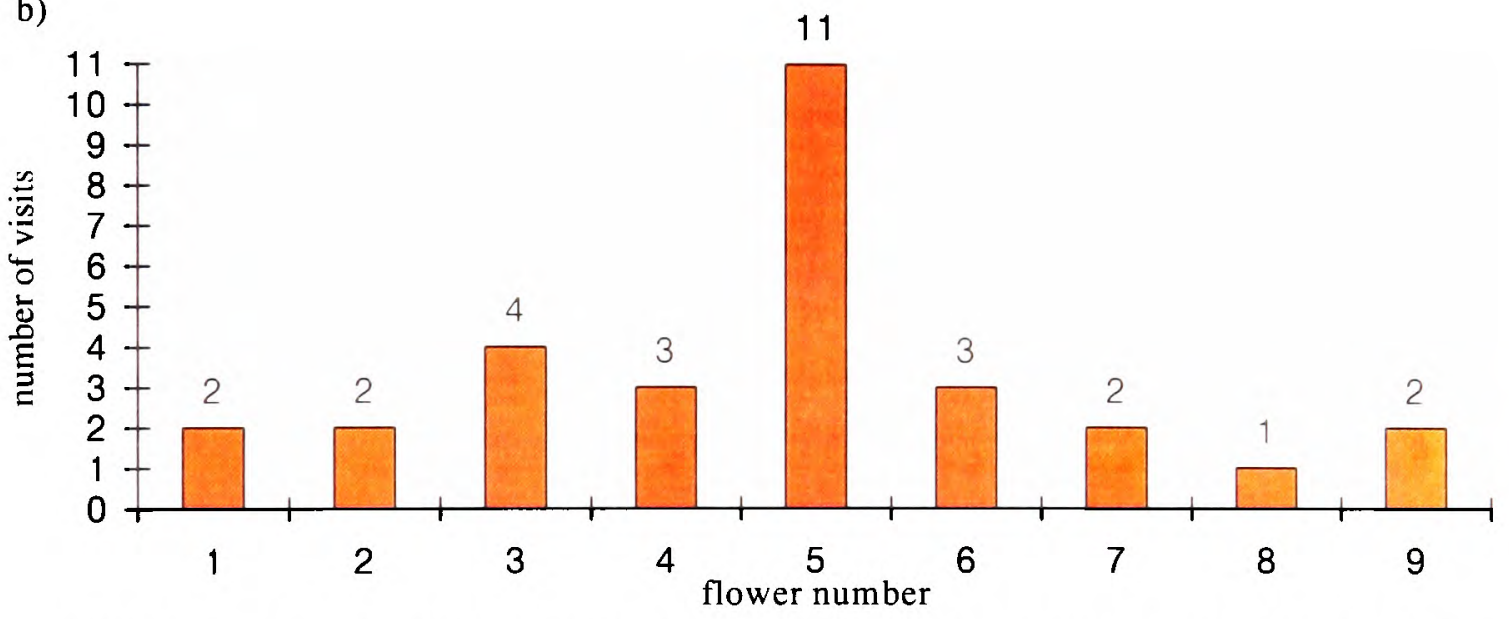

Figure 4-17 Visits of Papilio dardanus to an array of flowers with a) no other individuals present b) dead male pinned on flower 5.

Under control conditions, the preferences of the butterflies were not significantly different from random behaviour ( $p=0.384$, binomial test, $n=30$ ), whilst in the second they were significantly different ( $p=0.0021$, binomial test, $n=30$ ). Of course the probability of getting 11 or more visits to the precise flower on which the other butterfly was pinned is even less. The visits to the other flowers in the experimental array are not significantly different from random ( $p=0.711$, binomial test, $n=19$ ). So the butterflies appear to be choosing flowers at random when they are in an straight line, but when another individual is feeding on a flower they show a significant preference for that flower. It is interesting to note, however, that the preference for neighbouring flowers, seen in Experiment 4-4, appears not to be present. This may be related to the size of the flowers used, as these flowers were bigger than those used in Experiment 4-4. Their large size may indicate that there is likely to be enough nectar for two individuals to feed at the same time, or their size may simply make it easier for this to happen.

An analysis of the sexes of the butterflies involved (see Table 4-14), indicates that both sexes show the same trend. The behaviour of either sex in the control experiment is not significantly different from random (binomial tests, $\mathrm{p}=0.69$ for males, $\mathrm{p}=0.18$ for females, $\mathrm{n}=15$ in both cases). In 
the control experiment, the females' preference for the central flower is significant (binomial test, $\mathrm{p}=0.001, \mathrm{n}=16$ ), and approaching significance for the males (binomial test, $\mathrm{p}=0.06, \mathrm{n}=14$ ). Given the relatively small data sets in each case, it seems safe to conclude that there is not a significant difference between the behaviours of the two sexes.

\begin{tabular}{|c|c|c|c|c|c|c|c|c|c|c|}
\hline \multicolumn{2}{|c|}{ Flower number } & 1 & 2 & 3 & 4 & 5 & 6 & 7 & 8 & 9 \\
\hline Control & male & 0 & 4 & 2 & 3 & 1 & 2 & 1 & 0 & 2 \\
\hline & female & 2 & 1 & 1 & 2 & 1 & 5 & 1 & 1 & 1 \\
\hline Experimental & male & 1 & 0 & 2 & 3 & 4 & 1 & 2 & 0 & 1 \\
\hline & female & $\overline{1}$ & 2 & 2 & 0 & 7 & 2 & 0 & 1 & 1 \\
\hline
\end{tabular}

Table 4-14 The visits of Papilio dardanus of either sex to flowers in the control and experimental arrays of Experiment 4-6

\section{Discussion}

The presence of another individual feeding at an array of flowers appears to influence the feeding behaviour of other individuals of Papilio dardanus. The butterflies appear to match the behaviour of the individual already feeding, having a tendency to land on the same flower without showing any attraction towards the other individual as a mate or rival. It is not simply that the butterflies are being attracted by the sight of others to the feeding patch in general (as would be expected in most kinds of gregarious behaviour), but they are actually noting the exact feeding position of the other individual and copying that behaviour. From these experiments it is not possible to determine whether this has any effect on the butterflies' subsequent behaviour - i.e. whether they can be said to have learnt from the behaviour. However, given the rapid learning capabilities of butterflies as shown by Lewis \& Lipani (1990) and others, it would seem quite possible that the individuals could learn to feed from certain flowers if rewarded when copying feeding behaviour seen in other individuals. This capability could allow them to learn to identify rewarding flowers more quickly, and possibly also to learn how to find nectar within them.

This behaviour may not be true 'imitation', as it has not been shown that the butterflies have actually copied a behaviour and incorporated it into their behavioural repertoire, although they can be said to be showing 'social enhancement' (Galef, 1988). This type of behaviour has been shown once in invertebrates before - Fiorito \& Scotto (1992) showed that Octopus vulgaris will attack coloured balls having first seen a demonstrator do it in an adjacent tank. Although the example of Papilio dardanus feeding is not as impressive as the imitative behaviour of the Octopus, this appears to be the first demonstration of social facilitation in insects. In order to determine whether or not the butterflies are showing true 'imitation' behaviour (Whiten \& Ham, 1992) it would be necessary to design an experiment which would differentiate this from simply having their attention drawn to a food source. This would be difficult since firstly it is not possible to show that the butterflies are actually copying a behaviour pattern - the feeding reaction is innate and is carried out in the absence of other individuals 
- and secondly is not easy to show whether or not the choice of flower has been 'learnt' through the behaviour matching, as a subsequent test of the preferences of a butterfly which appeared to imitate on a first trial (in the presence of the other individual) would be greatly affected by the presence or absence of a reward on that first trial (an effect which would be difficult to remove).

\section{Conclusions}

The experiments in this chapter demonstrate that Papilio dardanus may have a relatively sophisticated feeding technique. They show a strong initial flower colour preference (preferring blue over yellow and red). This preference compares well with the experiments performed by Ilse (1928), Ilse \& Vaidya (1956) and Swihart (1970) on other Papilio species which all demonstrated a primary preference for blue flowers. Comparing the preference with the ERG carried out in Experiment 3-5 it can be seen that the red paint has a reflectance which will barely be visible to the butterflies (longer than $640 \mathrm{~nm}$ ). The yellow (longer than $580 \mathrm{~nm}$ ) and blue $(400-500 \mathrm{~nm}$ ) however, should both be clearly visible, exciting the pigments with peaks at $575 \mathrm{~nm}$ (which would make the 'yellow' pigment appear red-shifted compared with our perception of it, due to the lack of green and UV reflectance) and $475 \mathrm{~nm}$ (which would appear more green-shifted to them due to the lack of UV reflectance) respectively. Both these peaks are of a similar size, so the overwhelming preference for blue does not appear to be explained by the visual sensitivities of the butterfly. The preference, therefore (like the preference for orange found in Argynnis paphia in Chapter 2), must be due to a bias in higher processing. The fact that Crane (1955) observed tropical Papilionids and Pierids feeding mainly from red flowers in the field suggests that this initial preference for blue flowers is not adaptive. The preference for unpainted red flowers remains so far unexplained, but indicates that there is a factor other than colour which governs the innate preferences of the butterflies.

Experiment 4-1 demonstrated a high degree of colour constancy in the butterflies when the flowers offered a sugar water reward. It also presented a little evidence that if this reward was lacking, the butterflies were less constant, and tried other flowers. Experiments 4-4 and 4-5 also revealed that the butterflies could use social cues to improve their feeding success, trying a wider range of colours in Experiment 4-4 when another individual was present. This seemed to be due to butterflies feeding from flowers which were spatially close to that on which another individual appeared to be feeding regardless of the colour of the flower. Experiment 4-5 confirmed that the butterflies were indeed influenced by the presence of another individual feeding, but found that they were significantly more likely to feed off the same flower as another individual, rather than the neighbouring flower. The effect is likely still to be to increase the repertoire of colours from which an individual feeds, and possibly therefore the feeding efficiency, although Lewis (1986) found that increasing the number of species of flower from which an individual learnt to forage actually 
decreased their overall efficiency. This social effect is interesting, as it appears not to have been demonstrated before.

Cook et al. (1994) found a preference in male Papilio dardanus for yellow females over red females. In Experiment 4-1 there appeared also to be a preference for yellow over red (although the overwhelming preference for blue overshadowed this and made the two difficult to compare statistically), but the results of the ERG in Experiment 3-5 made it likely that this colour preference in flower choice was simply due to the visual sensitivities of the butterflies. Since Papilio dardanus shows a strong preference for blue flowers, it would be very interesting to test whether this colour preference could be present in their mate choice. Blue is a relatively unusual colour in nature as there are few blue pigments (although butterflies can use structural colours to produce blues (see Chapter 3)), but it is interesting to note that Papilio phorcas, the sister species of Papilio dardanus (Clarke et al., 1991;Vane Wright \& Smith, 1991; Vane Wright et al., in press) does possess a blue pigment, phorcabilin (Choussy \& Barbier, 1975) which it uses in association with yellow pigment to form its green coloration (Vane Wright et al., in press). This is discussed further in Chapter 5.

\section{Acknowledgements}

I am very grateful to Humbrol Ltd. for supplying the reflectance spectra for their enamel paints, and to Carol \& Tony Alden for the artificial flowers. I would also like to thank Phil Taylor for his help with the flight cage, and John Calvert at Stratford Butterfly Farm for providing the butterflies.

\section{$\underline{\text { References }}$}

Choussy, M. \& Barbier, M. 1975. Ptérobiline et Néoptérobilines, réactivité et structures. Helvetica Chimica Acta 58, 2651-2661.

Darwin, C. 1877. The effects of cross- and self-fertilization in the vegetable kingdom. Murray, London.

Clarke, C.A., Gordon, I.J., Vane-Wright, R.I., Smith, C.R. 1991. Phylogenetic relationships of three African swallowtail butterflies, Papilio dardanus, $P$. phorcas and $P$ constantinus: new data from hybrids (Lepidoptera: Papilionidae). Systematic Entomology 16, 257-273.

Cook, S.E., Vernon, J.G., Bateson, M., Guilford, T. 1994. Mate choice in the polymorphic African swallowtail butterfly, Papilio dardanus: male-like females may avoid sexual harassment. Anim. Behav. 47, 389-397.

Crane, J. 1955. Imaginal behavior of a Trinidad butterfly, Heliconius erato hydara Hewitson, with special reference to the social use of color. Zoologica (N.Y.) 40, 167-196.

Fiorito, G. \& Scotto, P. 1992. Observational learning in Octopus vulgaris. Science 256, 545. 
Frisch, K. Von. 1914. Der Farbensinn und Orientierung der Biene. Zool. J. Physiol. 37, 1-238.

Galef, B.G. 1988. Imitation in animals: history, definition, and interpretation of data from the psychological laboratory. In: Social learning. Psychological and biological perspectives (Ed. Zentall, T. \& Galef, B.). Hillsdale, New Jersey: Lawrence Erlbaum. 3-28.

Goulson, D. \& Cory, J.S. 1993. Flower constancy and learning in foraging preferences of the greenveined white butterfly Pieris napi. Ecol. Entomol. 18, 315-320.

Ilse, D. 1928. Über den Farbensinn der Tagfalter. Z. f. vergl. Physiologie Bd. 8, 658-692.

Ilse, D. \& Vaidya, V.G. 1956. Spontaneous feeding response to colours in Papilio demoleus L.. Proc. Indian Acad. Sci., Sect. B 43, 23-31.

Kandori, I. \& Ohsaki, N. 1996. The learning abilities of the White Cabbage butterfly, Pieris rapae, foraging for flowers. Res. Popul. Ecol. 38(1), 111-117.

Kandori, I. \& Ohsaki, N. 1998. Effect of experience on foraging behavior towards artificial nectar guide in the cabbage butterfly, Pieris rapae crucivora (Lepidoptera: Pieridae). Appl. Ent. and Zoo. 33(1), 35-42.

Kelber, A. 1996. Colour learning in the hawkmoth Macroglossum stellatarum. J. Expt. Biol. 199, 1127-1131.

Klein, R.M. 1978. Plants and near-ultraviolet radiation. Bot. Rev. 44, 1-127.

Lewis, A.C. 1986. Memory constraints and flower choice in Pieris rapae. Science 232, 863-865.

Lewis, A.C. \& Lipani, G.A. 1990. Learning and flower use in butterflies: hypotheses from honey bees. In Insect-Plant Interactions, Vol. II, ed. Bernays, E.A., CRC Press, Boca Raton, Florida. 95110.

Magnus, D. 1958. Experimentelle Untersuchungen zur Bionomie und Ethologie des Kaisermantels. $Z$. Tierpsychol. 15, 397-426.

Menzel, R. 1979. Spectral sensitivity and colour vision in invertebrates. In Handbook of Sensory Physiology Vol. VII/6A "Comparative physiology and evolution of vision in invertebrates" ed. Autrum, H., 503-580. Springer, Berlin.

Miller, N.E. \& Dollard, J. 1941. Social learning and imitation, New Haven: Yale University Press.

Scherer, C. \& Kolb, G. 1987. The influence of color stimuli on visually controlled behaviour in Aglais urticae L. and Pararge aegeria L. (Lepidoptera). J. Comp. Physiol. A 161, 891-898.

Swihart, C.A. \& Swihart, S.L. 1970. Colour selection and learned feeding preferences in the butterfly, Heliconius charitonius L.. Anim. Behav. 18, 60-64.

Swihart, S.L. 1970. The neural basis of colour vision in the butterfly Papilio troilus. J. Insect Physiol. 16, 1623-1636.

Swihart, C.A. 1971. Colour discrimination by the butterfly, Heliconius charitonius L.. Anim Behav. 19, 156-164.

Thorpe, W.H. 1963. Learning and instinct in animals. $2^{\text {nd }}$ Edition. London: Methuen. 
Vane Wright, R.I. \& Smith, C.R. 1991. Phylogenetic relationships of three African swallowtail butterflies, Papilio dardanus, $P$. phorcas and P. constantinus: a cladistic analysis (Lepidoptera: Papilionidae). Systematic Entomology 16, 275-291.

Vane Wright, R.I., Raheem, D.C., Cieslak, A., Vogler, A. In press. Evolution of the mimetic African swallowtail butterfly Papilio dardanus: molecular data confirm relationships with $P$. phorcas and P. constantinus. Biol. J. Linn. Soc.

Weiss, M.R. 1995. Associative colour learning in a nymphalid butterfly. Ecol. Entomol. 20, 298-301.

Weiss, M.R. 1997. Innate colour preferences and flexible colour learning in the pipevine swallowtail. Anim. Behav. 53, 1043-1052.

Whiten, A. \& Ham, R. 1992. On the nature and evolution of imitation in the animal kingdom: reappraisal of a century of research. Advances in the Study of Behaviour 21, 239-283. 


\section{Chapter Five}

\section{Mate choice in Papilio dardanus}

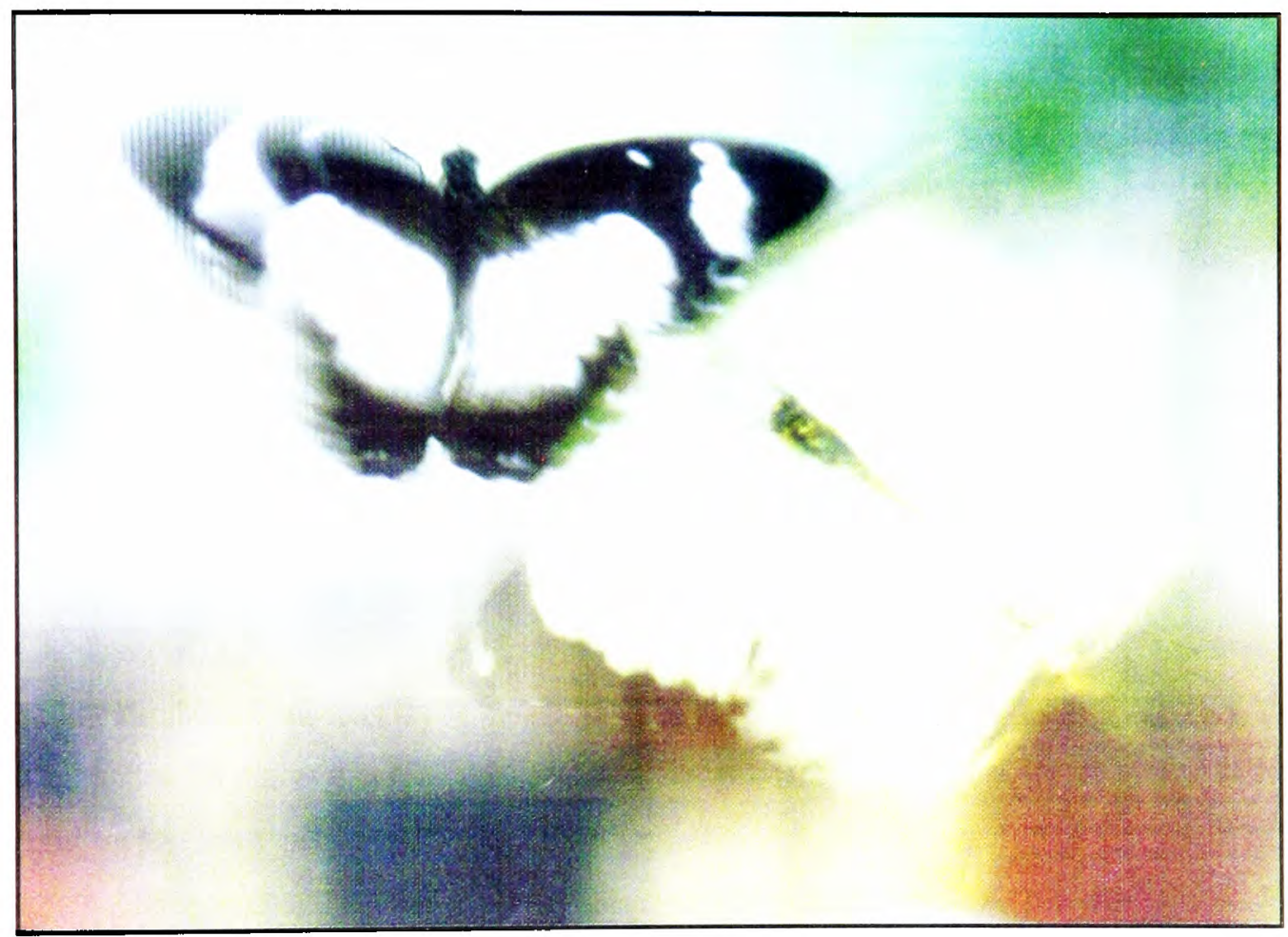

Figure 5-1 A hippocoonides female rejecting a male by landing with wings open, and fluttering them

"I find that a male lepidopteran has almost always beaten me to any given female"

Burns (1968) 


\section{Introduction}

Cook et al. (1994) studied male mate preferences in the tibullus race of Papilio dardanus found on Pemba, Tanzania. This population of the race tibullus has a tail-less male-like female (andromorph) called trimeni, the common black and white morph hippocoonides, which mimics Amauris niavius (Trimen, 1869), and both of these morphs can appear with orange on the hind-wings which are known as yellow and white lamborni, or prototrophonius and trophonius, respectively (Cook et al. 1994). See Appendix 1 for further details of the races and morphs, and Chapter 3 for further details about this population in particular. Cook et al. pinned out pairs of dead female morphs (and also males and Amauris niavius specimens) and recorded the number of 'approaches' by males (defined as when a male came within $30 \mathrm{~cm}$ of a pinned out female) to each morph, the amount of time each spent within $30 \mathrm{~cm}$ of the morph, and whether or not the male attempted to copulate with the female. They also repeated the experiment using only singly presented morphs to avoid possible interaction effects resulting from presenting two females simultaneously. Finally, they followed live females, and recorded the rate of male approaches whilst the different morphs were flying.

The first two experiments gave the same results: the males approached the black and white hippocoonides morph significantly more than trimeni or lamborni, and trimeni females were approached significantly more often than lamborni or old, faded, trimeni females. The difference between the number of approaches to trimeni and lamborni females versus pinned-out males was insignificant. Faded males were approached significantly less frequently than any of the other specimens, and Amauris niavius (the model for hippocoonides) was approached significantly less often than hippocoonides. Males frequently attempted to copulate with the pinned-out females, but never attempted to copulate with males or Amauris niavius. See Appendix 5 for further details on these results. The differences in interaction times that the males spent with each morph followed the same pattern. When Cook et al. followed flying females, they found that hippocoonides females were approached significantly more than trimeni females. The presence of a male preference would have very interesting consequences for the evolution of the species and the morphs, and would affect the balance of morphs in different populations.

Assuming that the polymorphism is balanced, there should be no selective advantage for males which show a preference for a particular morph as each morph must on average leave the same number of offspring. Therefore the results raise two questions. Firstly, how does the population remain in balance? This question is addressed in Chapter 7, but to do that it is necessary to answer the second problem - what is the basis of the observed male preference - and for this there are many possible explanations. Firstly, the black and white females could have been more conspicuous to the males simply due to their coloration contrasting more with the background. To test this, Bateson et al. (unpubl.) pinned hippocoonides and trimeni females on 'cryptic' or 'noncryptic' backgrounds (either 
plain card or card covered in leaf litter). They found that the preference for hippocoonides remained even when it was made apparently less conspicuous than the trimeni morph. However, the results of Chapter 3 show that the white patches on hippocoonides would be 'true white' (reflectant over their entire visual range) to the butterflies, and therefore they are likely to stand out against the non-UV reflective leaf litter. This is because they would differ both by a difference in hue (since Experiment 3-6 indicated that the butterflies possess red receptors which will not be stimulated by the green background) and brightness (Swihart, 1971 demonstrated that butterflies do detect differences in brightness). The trimeni morph, however, may well have the same degree of brightness as the background, and also their hue will appear shifted more towards green to the butterflies (if they possess true colour vision) due to the UV reflectance, whilst the non-UV reflectant background will remain a greenish coloration.

Another explanation could be that the males perceive the hippocoonides females as being more obvious due to some perceptual bias for their coloration in general. For this case to be demonstrated, it would have to be shown that there was no other basis for the preference. In Chapter 4, Papilio dardanus was shown to have an innate preference for blue flowers over red and yellow. It would therefore be interesting to test whether or not this preference was also shown in mate choice (as is the case in Argynnis paphia - see Chapter 2). However, in pilot tests, males would not react to dead or model females, even when made to rotate on a 'merry-go-round' apparatus, and spin on their axis to give a 'fluttering' effect as was done by Magnus (1958) for Argynnis paphia. Pilot tests in which hippocoonides females were artificially coloured using permanent markers also proved unsuccessful, as the females were never mated, even when they initiated vigorous courtship with the males. It is possible that this was due to the marker pen removing scent-scales from the wings of the females which would normally be brought into contact with the male during courtship and elicit a mating reaction. It was therefore not possible to investigate the presence of a 'hidden preference' for artificial blue morphs in the flight cage (although the fact that males reacted to dead pinned-out females in the field experiments by Cook et al. indicates that it might be possible to perform this test in the field).

A further possible explanation for the apparent bias for hippocoonides involves learning. Males have to be able to recognise females of their own species. Cook et al.'s experiments show that males of Papilio dardanus can do this by sight, although possibly only at close range, given that no males attempted to copulate with a pinned out male or female of the wrong species even though they were approached (the dead specimens would probably not have much residue of pheromones on them). It would be difficult for males of a polymorphic (and mimetic) race to recognise females of their own species by sight innately, and it is possible that males might learn to recognise their own species after their first mating. This would be similar to the learnt flower preferences in butterflies discussed in Chapter 4. Virgin males may approach females at random, but after one mating they may 
show a preference for females of the same morph as their first mate. Such a preference would explain the observed bias towards the most common morph, and can be tested experimentally using males with a known history of sexual encounters. So, the males may appear to show a preference for the commonest morph (whichever morph that is) simply because their past experiences are most likely to have been with this morph. Should the morph frequencies change, so would the males' apparent preference.

A final explanation for the popularity of the most common morph is that there is a genetic basis to male choice. Males carrying the genes for a morph may also carry the genes for preference for that morph (similar to that which appears to be present in Danaus chrysippus; Smith, 1984). If a gene controlling morph preference were to exist it would be expected to be in linkage disequilibrium with that for the morph coloration itself due to assortative mating between the carriers of the gene for coloration and that for the preference. If male choice were to be controlled by a gene for a morph preference, then the males would be expected to show the same preference all their life (although this may only be a preference, and they may mate with other morphs, though less frequently), and may provide similar data to the case for learning. The theory could easily be tested by determining the genetics of males with a certain preference. It is also possible that there is a gene for a morph preference which is not associated the gene for the morph itself, and to test this males could be allowed to mate only with one particular morph first, and then their subsequent choices studied.

In addition, there may be an element of female choice. The preferences of the males could be evolutionarily irrelevant if the females often refuse matings and their choice governs the mating patterns in the species (as again appears to be the case in Danaus chrysippus; Smith, 1984).

Butterflies often display female-limited polymorphism but never male-limited polymorphism. This has classically been explained in terms of female choice (Hespenheide, 1975; Turner, 1978; Silberglied, 1984). Females can refuse males, and therefore they exert a selection pressure on the males to conform to a monomorphic state. In the case of Papilio dardanus, the fluorescent yellow pigment, papiliochrome II, found in the males fades in sunlight to a non-fluorescent orange, and the wings also lose their UV reflectance (see Chapter 3 for further details). This would allow females to use an age-cue when selecting a mate. In addition, females may judge a male's fitness by the extent of his wing damage. A preference for younger males, judged by wing damage, has been found in Colias eurytheme (Rutowski, 1985). This is likely to be due to the fact that male butterflies have been found to produce a much larger spermatophore on their first than on any subsequent mating (Svärd \& Wicklund, 1986). Thus female selection for lack of wing damage could be an explanation for the tails found in many swallowtail species. These break off very easily, and thus could be an honest signal of quality - females may prefer symmetrical males with two tails over those with one tail. It is not clear whether females would thus prefer symmetrical males with no tails over males with 1 tail, and this could also be tested using choice tests. Symmetry itself may also be preferred, either as an indicator 
of quality (Parsons, 1990), or as the product of another perceptual bias (Johnstone, 1994; Enquist \& Arak, 1994).

\section{$\underline{\text { Aims }}$}

The aims of this chapter are:

- To determine whether male Papilio dardanus have any initial preferences in their selection of a female morph as a mate (as found in their overall mate choices by Cook et al. 1994).

- To investigate whether there is any correlation between the initial choice of morph by a male and his subsequent choices, and (if there is) to try to determine whether this is due to learning or a genetic effect.

- To determine whether female Papilio dardanus show any preference for males with both swallowtails intact, or for symmetrical males.

The results from this chapter should suggest how the males' preference, as observed by Cook et al. (1994), might come about - whether it is a genetic effect, the result of learning, or whether it is likely to be due to a perceptual bias.

\section{Behavioural Observations}

All behavioural experiments were carried out in a flight cage measuring $3.5 \mathrm{~m} \times 1.5 \mathrm{~m} \times 2.4 \mathrm{~m}$ in a greenhouse at $30-40^{\circ}$ Centigrade. In the cage were placed tall plants (including a Citrus) - see Appendix 3 for further details. Without these plants, and at lower temperatures, the butterflies rarely flew. At higher temperatures, mortality in the butterflies increased.

Butterflies usually took about an hour after being placed in the flight cage before flying around, and females usually refused matings until they had acclimatised for 3 or 4 hours. Males and females often flew at each other as well as members of the opposite sex. Landed males and females were rarely approached, but where they were the approaching butterfly (whether male or female) would fly repeatedly at the landed individual from below, touching the upper surfaces of the landed butterfly's wings with its own. When females approached males (or females) in the air, they flew up at the undersides of the butterfly repeatedly, brushing the upper surfaces of their wings against the lower surfaces of those of the male. Males did the same when approaching females, and if the female accepted, the pair would land together. If accepting, the female curved her abdomen under slightly at the tip, and the males would land next to her, curving his abdomen under to mate with the female. As 
soon as he had clasped her, the male would drop motionless, to be carried in flight by the female if the pair should be disturbed. Often the female would land and curve her abdomen, but the male would appear to lose sight of her once she had stopped flying, and occasionally he would even land next to her, and perhaps curve his abdomen under, but would not proceed any further with copulation if he did not manage to mate with her immediately. Females would sometimes chase and land with other females, and occasionally one would land on the other as the males do, but none actually attempted copulation as males do. If the mating were successful, the pair remained in copula for about one hour before separating. Females which rejected males would either change their wingbeat pattern to a fast, fluttering flight, at which the males would fly off and leave them, or land and flap their wings vigorously to prevent approach. If pestered consistently by several males, a female would, rarely, drop to the ground and remain still. The sight of a pair in mid-air often attracted other males, and usually the males would be distracted by and pursue each other, ignoring the female.

Males appeared uninterested in females on the day that they emerged, although females would sometimes mate on their first day despite being generally less active. On the second day, both males and females were ready to mate, and on the third day both pestered each other vigorously. After about 4 days, however, the females started to lay eggs (whether they had been mated or not). When doing this they adopted a very fluttering flight, and laid up to 70 or so eggs on the underside of Citrus or Choisya leaves.

\section{Experiment 5-1 Initial morph preference of male Papilio dardanus}

\section{Introduction}

Cook et al. (1994) reported morph preferences in wild males. This preference could be innate (due to a genetic or perceptual bias) or learned, so this initial experiment was simply to allow naïve males to mate with a female of their choice having had no previous experience of females and being presented with even numbers of each morph. Matings of Papilio dardanus in suitable flight cages have been reported (Stride, 1958), so such mate choice experiments could be carried out in a suitable heated greenhouse. If a preference for hippocoonides was found in these naive males then it could be assumed that the preference was not learned, but was in fact an innate preference - either a genetically determined preference for a particular morph or due to a perceptual bias for the coloration in general. If no preference was found then it is possible either that the behaviour of the wild males is due to experience or that the behaviour in the flight cage is not the same as that displayed in the field due to factors such as the size of the cage or a difference in the relative conspicuousness of the morphs. 
Aim

The aim of this experiment is to determine whether or not naïve male Papilio dardanus show a morph preference when choosing mates.

\section{Method and Materials}

Since individual Papilio dardanus from race tibullus, as studied by Cook et al. (1994) could not be obtained in England in the quantities necessary for experimentation, the geographically neighbouring race, meseres, was used (see Appendix 1 for details of the races). The butterflies were sent as pupae weekly, and for the major part of the experimental work (June 1997-September 1998) 20-30 pupae were received each week from a total batch of about 400 per week from the supplier in Moshi, Tanzania. This meant that the individuals were as near unrelated as was practically possible. Unfortunately the andromorph, trimeni, which was present in the Pemba population of race tibullus, used by Cook et al., was not present in this race. Instead a mimetic morph, cenea, was relatively common, and was used as the third option in the mate choice experiments (see Appendix 1 for further details of the morphs).

Males and females were kept in separate nets when they emerged. Virgin males (up to 11 at any one time) were placed in the flight cage together with equal numbers of virgin females of each morph. The numbers of females in the net were kept as high as possible (whilst maintaining equal numbers of each morph) so as to increase the chances of a meeting between the sexes and also decrease the possibility of individual differences amongst the females affecting the results. There were generally between 1 and 3 females of each morph present. The ages of the females were kept as close as possible (and certainly there was no more than a 4 day difference between their emergence dates). Where all three morphs of female were not available, experiments were done with males having only a choice of females from two morphs.

Once a mating had occurred between two butterflies, the pair was placed in a separate net, and the numbers of different female morphs made equal again. The two mated individuals were marked on the undersides of the wings with a permanent marker so that they would not be mixed up with unmated individuals. The female morph that was chosen by each male was noted. The mated females were then placed in laying cages with sprigs of Choisya ternata (the Mexican Orange Flower), and the males kept for further experimentation (see Experiment 5-2). 


\section{Results}

Males with a choice of all three morphs:

\begin{tabular}{|c|c|c|}
\hline Hippocoonides chosen & Cenea chosen & Lamborni chosen \\
\hline 7 & 4 & 8 \\
\hline
\end{tabular}

Table 5-1 The morph choices of naïve male Papilio dardanus in a three-way choice test

The results in Table 5-1 indicate that the males show no significant preferences amongst the morphs $\left(\mathrm{p}=0.504, \chi^{2}=1.37, \mathrm{n}=19\right)$.

Males with a choice of hippocoonides and cenea:

\begin{tabular}{|c|c|}
\hline Hippocoonides chosen & Cenea chosen \\
\hline 12 & 8 \\
\hline
\end{tabular}

Table 5-2 The morph choices of naïve male Papilio dardanus given a choice of hippocoonides and cenea females

The males appear not to show a significant preference for either hippocoonides or cenea in a pairwise comparison $(\mathrm{p}=0.252$, binomial test, $\mathrm{n}=20)$.

Males with a choice of cenea and lamborni:

\begin{tabular}{|c|c|}
\hline Cenea chosen & Lamborni chosen \\
\hline 3 & 2 \\
\hline
\end{tabular}

Table 5-3 The morph choices of naïve male Papilio dardanus given a choice of lamborni and cenea females

Males with a choice of lamborni and hippocoonides:

\begin{tabular}{|c|c|}
\hline Lamborni chosen & Hippocoonides chosen \\
\hline 4 & 2 \\
\hline
\end{tabular}

Table 5-4 The morph choices of naive male Papilio dardanus given a choice of lamborni and hippocoonides females

The data in Table 5-3 and Table 5-4 do not show any obvious preferences, but form data sets too small for statistical analysis.

\section{Discussion}

The results show that the males had no significant preference between the different morphs. This is very different from the results obtained in the field by Cook et al. (1994). There are several possible explanations for this difference:

Firstly, these results were obtained in a small flight cage, and it is possible that the behaviour shown in this cage is not representative of the behaviour of the species in the wild. This could be because there is not a significant difference between the conspicuousness of the three morphs in the relatively small, black mesh net, or that the density of butterflies is simply unrealistically high 
(although this latter explanation seems less likely as they did not encounter each other particularly often, and mating was relatively rare).

Secondly, it is possible that since only matings are being recorded in this experiment, and not approaches by males to females (as was recorded in Cook et al.'s experiments), then two different phenomena are being compared. As far as the dynamics of the morph populations are concerned, number of matings are all that matter, but it could still be that the males have a preference for hippocoonides which is over-ridden by the females themselves, who determine whether or not mating occurs.

Thirdly, Cook et al. were studying experienced, wild, males who may well have mated previously. So it is possible that these males have mated at random on their first encounter with a female (which is most likely to have been a hippocoonides since it is most frequent in the study population) and have learned from that mating to recognise hippocoonides females as mates, thus causing the preference for hippocoonides observed in the field experiments. Experiment 5-2 aims to investigate this hypothesis by testing whether or not male Papilio dardanus are influenced by previous experience.

These results also do not include male preferences for the andromorphic females. It is therefore not possible to test the hypothesis that these females are either particularly attractive to males (as suggested by Vane-Wright, 1984, in the "pseudosexual selection" hypothesis) or are not attractive to males (as suggested by Cook et al. 1994). Male behaviour towards other males in the flight cage (as described in the "behavioural observations" section of this chapter) suggests that they may approach male-like females more often than others, as they often followed other males rather than mimetic females. This issue is discussed further in Chapter 7.

\section{Experiment 5-2 Second morph choice of male Papilio dardanus}

\section{Introduction}

As discussed in the introduction to this chapter, the males' preference for the most common morph, as demonstrated by Cook et al. (1994), could be due to a genetic or learnt preference. An influence of experience has been found in many vertebrate and invertebrate species - in some cases mating experience itself appears to increase a male's chance of mating (Cook, 1995; Pomiankowski, 1990; Clutton-Brock et al., 1989; Burley \& Moran, 1979), or non-sexual interactions with other individuals (often parents or siblings) influence the future choice of mate (see Table 5-5). In others an 
individual's past sexual experience actually seems to affect their choice of mate (see summary in Table 5-6).

Experiment 5-1 appears to indicate that the preference might be learnt, as the naïve males seem to be choosing at random, rather than showing the preferences illustrated by Cook et al. If the preference is simply due to the hippocoonides morph being more likely to be noticed by the males, then it would be expected that the morphs would be preferred in the same proportions as in Experiment 5-1, but that there would not

\begin{tabular}{|c|c|}
\hline Animal & Reference \\
\hline rodents & see review by D'Udine \& Alleva, 1983 \\
\hline birds & Aberle et al., 1963 \\
\hline & Cooke \& Davies, 1983 \\
\hline & Immelmann, 1985 \\
\hline & Vos, 1995 \\
\hline fish & Haskins \& Haskins, 1950 \\
\hline & Liley, 1966 \\
\hline & Ferno \& Sjölander, 1973 \\
\hline & Kop \& Heuts, 1973 \\
\hline & Sjölander \& Ferno, 1973 \\
\hline & Barlow et al. 1990 \\
\hline & Breden et al., 1995 \\
\hline
\end{tabular}

Table 5.5 Summary of works relating to the influence of nonsexual encounters on mate choice be a significant correlation between an individual's choice of first and second mates. However, if there is indeed a genetic or learnt preference, there should be an increased probability of males choosing the same morph on their second mating as they did on their first.

\begin{tabular}{|c|c|c|c|}
\hline Species & Common name & Preference & Reference \\
\hline Taeniopygia guttata & zebra finch & $\begin{array}{c}\text { females increased preference for males with } \\
\text { high display rate if exposed previously to low } \\
\text { display rate and vice versa }\end{array}$ & Collins, 1995 \\
\hline Ficedula hypoleuca & pied flycatcher & $\begin{array}{c}\text { females may return to and mate with } \\
\text { previously visited males }\end{array}$ & Dale et al., 1995 \\
\hline Cottus bairdi & mottled sculpin & $\begin{array}{c}\text { females choose male which is at least as large } \\
\text { as previously encountered }\end{array}$ & Brown, 1981 \\
\hline $\begin{array}{c}\text { Gasterosteus } \\
\text { aculeatus }\end{array}$ & stickleback & $\begin{array}{c}\text { females choose male which is more intensely } \\
\text { red than previously encountered }\end{array}$ & $\begin{array}{c}\text { Bakker \& Millinski, } \\
1991\end{array}$ \\
\hline
\end{tabular}

Table 5-6 Summary of effects of past sexual experience found on mate choice in different species

\section{Aim}

This experiment aims to determine whether or not males are more likely to choose the same morph on their second mating as they did on their first than if they were selecting at random.

\section{Method and Materials}

Experiments were carried out in the same net as described in Experiment 5-1 (see Appendix 3 for further details).

Males which had already made one recorded mating (in Experiment 5-1) were placed singly in the flight cage together with equal numbers of virgin females of each morph which was available to the male for his first choice. The numbers of females in the net were kept as high as possible (whilst 
maintaining equal numbers of each morph) so as to increase the chances of a meeting between the sexes and also decrease the possibility of individual differences amongst the females affecting the results (generally between 1 and 3 individuals of each morph were present). The ages of the females were kept as close as possible (and there was never more than a 4 day difference in their ages).

Once a mating had occurred between two butterflies, the pair was placed in a separate net, and the numbers of female morphs made even again. The female morph that was chosen by each individual male was noted, and the pair were marked on the underside of their wings with a permanent marker to ensure that they could be recognised. The females were then placed in laying cages with sprigs of Choisya ternata (the Mexican Orange Flower).

\section{Results}

\begin{tabular}{|l|c|c|}
\hline & Chose same morph & Chose different morph \\
\hline Choice of 3 morphs & 6 & 2 \\
\hline Choice of 2 morphs & 2 & 7 \\
\hline
\end{tabular}

Table 5-7 The morph choices of experienced male Papilio dardanus given a choice of either two or three morphs each time

Using a simple binomial probability test, the data from the choice of three morphs shows that the males choose the same morph on their second mating significantly more often that they choose either of the other two ( $p=0.0197$, binomial test, $n=8$ ). The data from the choice of two morphs shows no significant preference for either the same morph or the other $(\mathrm{p}=0.090$, binomial test, $\mathrm{n}=9$ ), and so more data would be required to demonstrate any preference.

\section{Discussion}

From the data from males who had a choice of all three morphs, it appears that males do show a pattern in their mating preferences and that they choose the same morph on their second mating as on their first significantly more often than choosing at random. This means that learning from a first mating could be the explanation for the difference seen between the field data of Cook et al. (1994) from experienced males and the mate choice data recorded in Experiment 5-1. In Chapter 4 it was shown that individual Papilio dardanus showed $90 \%$ constancy to the colour of the first flower from which they fed, indicating that they could learn from one experience. It appears that this may be a further demonstration of such a capability. The data from males who had a choice of only two morphs is less clear - it even appears to show a (not significant) bias for the morph opposite to that chosen in the first experiment. More data would be required on this experiment to determine whether or not the results for the two-way choice are indeed different from those for the three-way choice. It was unfortunately not possible to gather more data on either of these experiments due to the restraints of time and supply of butterflies. Therefore the significant results of the three-way choice were accepted, although this result may not stand when further data is gathered. 
It is still possible that the consistent choice of one morph by males is genetically determined. In fact, this could even be supported by the difference between the two-way and three-way choices in this experiment as males with a choice of only two morphs may not be able to demonstrate their innate morph preference, and thus may be showing different behaviour from those which have a choice of all three morphs and are therefore always able to choose their preferred morph. This is investigated in Experiment 5-3.

\section{Experiment 5-3 Genetics of males showing morph preferences}

\section{Introduction}

It is possible that the morph preferences demonstrated by Cook et al. (1994) have a genetic component, with the 'gene for preference' in linkage disequilibrium with the genes for the pattern formation (as it is likely to be, due to matings between carriers of the morph gene and those carrying the gene for the preference). This assortative mating could explain why most males preferred females carrying the most common morph gene. It could also explain the morph constancy demonstrated in Experiment 5-2 (and the possible anomaly of the two-way choice data being different from the threeway choice data in Experiment 5-2), although it is rendered less likely by the results of Experiment 51 , where males appeared to be choosing mates at random despite the fact that the hippocoonides morph was most common, indicating that the recessive hippocoonides gene was the most common gene.

Since the genetics of the morphs are well understood (Clarke \& Sheppard 1959, 1960a, 1960b, 1962 and see Appendix 1) it is possible in a lot of cases to calculate the genes carried by the males if the morph of the mother is known (for example, in all cases where the mother is hippocoonides, the bottom recessive). Thus, by allowing males to choose freely, and then noting the morphs present in their offspring it should be possible to assess the presence of any genetic effect associated with the morph genes they carry.

\section{Aim}

The aim of this experiment is to determine whether or not males carrying the genes for a particular morph also show a preference for that particular morph when choosing a mate. 


\section{Method and Materials}

Females which had been chosen as mates by males given a choice of all three morphs in the free-choice experiment, Experiment 5-1, were left to lay in cages containing Choisya ternata. Any resulting offspring were kept in separate fine-mesh cages, and the female morphs which emerged were noted. This allowed the pattern genes carried by the male to be calculated in most cases.

In addition, Ford (1936) collated the results of broods laid by wild-caught females. These can give a clue to behaviour in the wild. Since all the matings were the result of free choice in the wild, this data can also be used to assess the possibility of a genetic influence on the morph choices of males.

\section{Results}

1) Results from flight cage matings:

Only five broods were successfully raised to emergence separately. The results of these broods are shown in Table 5-8.

\begin{tabular}{|c|c|c|c|c|c|c|}
\hline male choices & $\begin{array}{c}\text { female } \\
\text { morph }\end{array}$ & $\begin{array}{c}\text { female } \\
\text { gene 1 }\end{array}$ & $\begin{array}{c}\text { female } \\
\text { gene 2 }\end{array}$ & offspring & $\begin{array}{c}\text { male } \\
\text { gene 1 }\end{array}$ & $\begin{array}{c}\text { male } \\
\text { gene 2 }\end{array}$ \\
\hline $2 \times$ hippocoonides & hippocoonides & $\mathrm{h}$ & $\mathrm{h}$ & $14 \times$ lamborni & $\mathrm{T}$ & $\mathrm{T}(?)$ \\
\hline $2 \times$ lamborni & lamborni & $\mathrm{T}$ & $?$ & $2 \times$ lamborni & $?$ & $?$ \\
\hline $1 \times$ hippocoonides & hippocoonides & $\mathrm{h}$ & $\mathrm{h}$ & $\begin{array}{c}5 \times \text { hippocoonides, } \\
6 \times \text { cenea }\end{array}$ & $\mathrm{C}$ & $\mathrm{h}$ \\
\hline $\mathrm{1}$ × hippocoonides & hippocoonides & $\mathrm{h}$ & $\mathrm{h}$ & $1 \times$ hippocoonides & $\mathrm{h}$ & $?$ \\
\hline $\begin{array}{c}\text { 1 } \times \text { lamborni, } \\
\text { 1 }\end{array}$ & lamborni & $\mathrm{T}$ & $?$ & $8 \times$ lamborni & $?$ & $?$ \\
\hline
\end{tabular}

Table 5-8 The results of broods raised after males had been given free choices of all three morphs. The first column shows the morphs the father of the brood chose in choice experiments, the second gives the morph of the mother of the brood. The third and fourth columns give the morph genes carried by the mother (where it is possible to determine them), the fifth column lists the female offspring of the brood, and hence the morph genes carried by the father can sometimes be determined (the last two columns)

The last two columns of Table 5-8 indicate that this experiment is not very informative. In only one case are both the male genes known for certain, although in the first brood shown in Table 58 both genes are probably the same given that the brood only contained lamborni females. If the father of the first brood did not carry a hippocoonides gene, then the choices cannot be strictly guided by a gene linked to those for morph as the male chose hippocoonides over lamborni twice in the choice experiments. The second and fifth broods in the table are completely uninformative as it is not possible to determine either of the genes carried by the male. The father of the third brood must have carried both a hippocoonides gene and a cenea gene, and that of the fourth brood at least one hippocoonides gene, and both males chose a hippocoonides female in a choice test. However, there is simply not enough data to analyse. 
2) For the results from Ford (1936), see Appendix 4.

This data is very difficult to analyse, as so many of the second genes are not known (due to small broods, or dominance of other genes). It is possible, however, to compare the proportions of the genes calculated to be present in the males who mated with each female morph with the proportions of the genes in the populations from which they came (also recorded by Ford). This is done in Appendix 4 , and the two samples which were large enough to allow a statistical analysis showed that the gene frequencies of the mated males do not differ significantly from those in the overall population.

One further test, which would allow an analysis of a greater proportion of the data, is to compare the ratio of homozygotes to heterozygotes in the wild population. Since the genotypes of the females are known completely in many cases, the proportion of homozygotes represented by them can be compared with that predicted by random mating. This is also done in Appendix 4. For race polytrophus, the predicted numbers of homozygotes versus heterozygotes is 21.7 versus 27.4 , whilst the observed numbers are 16 and 33 . Thus the observed ratio is not significantly different from that predicted by random mating (binomial test; $p=0.919, n=29$ ). For race cenea, the predicted numbers of homozygotes and heterozygotes are 7.5 and 6.5 respectively, and the observed values are 5 and 9 . A binomial test on these results indicates that they do not significantly deviate from those expected if mating was at random (binomial test; $p=0.152, n=14$ ). It can therefore be said that the ratio does not indicate that males preferentially mate with females carrying the same genes (as indicated by their morph) as themselves. The slight bias towards heterozygotes in the observed data as compared with the expected values may have two contributing factors. Firstly, biases in the collecting of the females (for example, not catching as many hippocoonides females as others), would bias the ratio of homozygotes (e.g. hippocoonides) to heterozygotes. Secondly, the cases where the genotype of the female is not known (and therefore the female is left out of the analysis) are more likely to be cases where the female was homozygote (and therefore the second gene was 'masked' by the first) than when it was heterozygote (when both genes may be become obvious from the morphs apparent in the resulting brood).

\section{Discussion}

From the evidence of the random mate choice in Experiment 5-1 and the calculation of the parentage of the broods reported by Ford (1936) it appears very unlikely that there is a genetic component to the males' mate choice which is associated with the genes for the patterning itself. It therefore appears, if the results of the three-way choice in Experiment 5-2 are accepted, that the males do indeed 
learn from their first mating and subsequently choose the same morph with a higher probability. This has implications for the population dynamics of the morphs, which is investigated in Chapter 7.

\section{Experiment 5-4 Female preferences for symmetry and swallowtails}

\section{Introduction}

The presence of swallowtails on the hindwings of many of the species in the genus Papilio has not been explained. In Papilio dardanus, the mimetic females have on the whole completely lost their swallowtails in order to mimic their un-tailed model species (a few tailed mimics have been reported from Kenya (Ford, 1936), and the rare mimetic females in race antinorii from Ethiopia are tailed (Trimen, 1869)), but the maintenance of the tails in the males and the non-mimetic females of races antinorii, meriones and humbloti implies that there is some advantage to having tails. One explanation for the presence of tails would be that they provide an aerodynamic advantage, but little work has been done on butterfly flight (Chai \& Srygley, 1990) and so this has yet to be studied. It is also possible, since the tails are extremely delicate and easily broken off, that they are used in signalling. The presence of strong female choice in sexual selection is often cited as a reason for the abundance of female-limited and absence of male-limited polymorphism in butterflies (Belt, 1874; Turner, 1978; Silberglied, 1984; Hespenheide, 1975). Female choice has only rarely been studied however, and it has been shown that the females appear to choose younger males, possibly on the basis of the extent of their wing damage (Rutowski, 1985).

Females have been shown to prefer symmetry when choosing mates in some species (swallows - Møller, 1992; humans - Thornhill \& Gangestad, 1993; zebra finches - Swaddle \& Cuthill, 1994), and symmetry has been shown to be correlated with mating success is others (peacocks Manning \& Hartley, 1991; scorpionflies - Thornhill, 1992; earwigs - Radesäter \& Halldórsdóttir, 1993; damselflies - Harvey \& Walsh, 1993; midges - McLachlan \& Cant, 1995; field crickets Simmons, 1995; dung flies - Allen \& Simmons, 1995; cerambycid beetles - Møller \& Zamora-Muñoz, 1997). Some studies have shown conflicting evidence, or no symmetry preference (paradise whydahs - Oakes \& Barnard, 1994; red-winged blackbirds - Dufour \& Weatherhead, 1998; fruit flies - Markow \& Ricker, 1992), but a review and analysis by Møller \& Thornhill (1998) concluded that there was an overall correlation between symmetry and female preference. There is much debate as to whether this represents a perceptual bias (Johnstone, 1994; Enquist \& Arak, 1994) or an adaptive choice to avoid 'fluctuating asymmetry' (FA, defined as deviations from perfect symmetry which are normally distributed around a mean of zero: Van Valen, 1962), which can indicate a degree of poor adaptation to the environment (see Parsons, 1990, for a review of the evidence for this). Some insects have been 
shown to have a preference for symmetrical flowers when feeding (Møller \& Sorci, 1998 although Giurfa et al., 1996, did not find this in bees), which is difficult to explain as a selected trait as there is currently no reason to believe that asymmetrical flowers should provide a lesser reward for nectar feeders. In swallowtail butterflies a female preference for symmetry per se might lead to females preferring males either with no tails at all or both tails intact, whereas a preference for a young, healthy male should lead to females preferring only males with both tails present over males with either one or both missing. If females are selecting their mates on the basis of their tail condition, it should therefore be possible to distinguish between these two mechanisms.

\section{Aim}

The aim of this experiment is to determine whether females preferentially mate with males with either both swallowtails intact, or symmetrical wings (either with or without swallowtails).

\section{Method and Materials}

Because of the comparative rarity of matings obtained in the flight cage, matings obtained in Experiment 5-1 were used as the basis for this experiment. When matings occurred in Experiment 51 , where virgin males were placed in the flight cage with virgin females and allowed to mate (see Experiment 5-1 for further details), the wing condition of all the males in the cage as well as the male with which the female mated were assessed and recorded (the wing conditions of the males were not artificially altered in any way). The males were recorded as either being '2-tailed' (where both swallowtails on the males' wings were intact), '1-tailed' (where only one swallowtail was intact) or '0-tailed' (where neither were present). In addition, some experiments were carried out in exactly the same manner when equal numbers of each female morph were not available, which were not part of Experiment 5-1. In these cases the numbers of each condition of male were kept as even as possible, although the fact that the males broke their tails during the experimental time meant that at the time of mating (when their condition was assessed) they were not necessarily evenly distributed amongst the conditions.

\section{Results}

\begin{tabular}{|l|l|l|l|l|}
\hline \multirow{2}{*}{ female number } & male chosen & 2-tailed males & choice \\
& 2-tailed males & 0-tailed males \\
\hline 1 & 2-tailed & 1 & 1 & 1 \\
\hline 2 & 0 -tailed & 0 & 1 & 4 \\
\hline 3 & 1-tailed & 0 & 3 & 2 \\
\hline 4 & 0 -tailed & 0 & 1 & 5 \\
\hline 5 & 0 -tailed & 0 & 1 & 4 \\
\hline 6 & 0 -tailed & 0 & 1 & 3 \\
\hline 7 & 2-tailed & 8 & 3 & 1 \\
\hline 8 & 0 -tailed & 7 & 3 & 1 \\
\hline 9 & 2-tailed & 7 & 3 & 0 \\
\hline
\end{tabular}




\begin{tabular}{|c|c|c|c|c|}
\hline 10 & 2-tailed & 1 & 4 & 2 \\
\hline 11 & 0 -tailed & 3 & 3 & 2 \\
\hline 12 & 2-tailed & 1 & 2 & 2 \\
\hline 13 & 1-tailed & 0 & 2 & 2 \\
\hline 14 & 0 -tailed & 2 & 1 & 1 \\
\hline 15 & 1-tailed & 2 & 1 & 0 \\
\hline 16 & 2-tailed & 2 & 1 & 1 \\
\hline 17 & 1-tailed & 1 & 1 & 1 \\
\hline 18 & 0 -tailed & 3 & 1 & 2 \\
\hline 19 & 0 -tailed & 1 & 1 & 2 \\
\hline 20 & 0 -tailed & 1 & 1 & 3 \\
\hline 21 & 2-tailed & 1 & 1 & 2 \\
\hline 22 & 1-tailed & 1 & 2 & 3 \\
\hline 23 & 2-tailed & 2 & 2 & 3 \\
\hline 24 & 2-tailed & 1 & 1 & 3 \\
\hline 25 & 0 -tailed & 0 & 1 & 3 \\
\hline 26 & 1-tailed & 3 & 2 & 1 \\
\hline 27 & 1-tailed & 0 & 2 & 1 \\
\hline 28 & 1-tailed & 3 & 5 & 2 \\
\hline 29 & 1-tailed & 1 & 2 & 2 \\
\hline 30 & 0-tailed & 3 & 3 & 2 \\
\hline 31 & 1-tailed & 3 & 3 & 2 \\
\hline 32 & 1-tailed & 0 & 1 & 1 \\
\hline 33 & 0 -tailed & 0 & 1 & 2 \\
\hline 34 & 2-tailed & 5 & 2 & 2 \\
\hline
\end{tabular}

Table 5-9 The results of females choosing males with differing numbers of swallowtails on their hindwings

The uneven numbers in each experiment, and the fact that in some cases one class had no representatives, make an analysis of the data more difficult than usual. However, it is possible to analyse it on the basis of males making a binomial choice. This was done using a short computer program written in $\mathrm{C}$ (see Appendix 6) which calculated the probabilities of all the possible outcomes of the experiment, and summed these for all the combinations in which each possible observable result could have been obtained (since the order in which the data points were gained is irrelevant). The probability that exactly these results were gained by chance is thus the number of possible ways in which the results could have been obtained over the number of possible results, and the p-value can be calculated as the probability that this result, or a more extreme one, could have been obtained by chance.

This method gave a p-value of 0.64 for females preferring symmetrical males (either 2-tailed, or 0-tailed) over non-symmetrical males (1-tailed), and $\mathrm{p}=0.44$ for females preferring perfect, 2-tailed males over any other class of male. Thus females do not appear to have a significant preference for symmetrical or intact males. 


\section{Discussion}

Although the statistical method used is not powerful (and therefore it is possible that a trend in the data exists which is not detected), the high p-values indicate that there is unlikely to be any sizeable trend in the data. It therefore appears that females are not mating preferentially with males on the basis of the condition of their swallowtails, or choosing symmetrical males. It thus seems likely that the swallowtails found on the hind-wings of the males are not sexually selected ornaments, and are more likely to be used either as a defence (perhaps as a means of escaping bird attacks) or are present for their aerodynamic effect.

These results do not mean that females are not selecting males on the basis of other characteristics, however. As Rutowski (1985) found, some female butterflies preferentially mate with younger males, who are likely to be more fertile (Svärd \& Wicklund, 1986). In the case of Papilio dardanus, the fading of the males to a non-UV reflectant orange (Cook et al., 1994, and see Chapter 3) could act as a very good cue of age. The colour and patterning of Papilio dardanus differs remarkably from the closely related species, Papilio phorcas and Papilio constantinus (Clarke et al., 1991; VaneWright \& Smith 1991), and it is possible that this change has been due to a sexual selection pressure by the females. It would be very interesting to test the hypothesis that females prefer unfaded males over faded males which appeared to be older. However, to do this it would be necessary to fade males artificially (they do not fade within their own lifespan in normal English weather), and this proved impossible. Experiment 3-7 indicated that the fading was due to wavelengths between $400-475 \mathrm{~nm}$, and it was not possible to find a lamp with high enough power at these wavelengths to cause fast artificial fading in live butterflies, and therefore it was not possible to test this hypothesis.

\section{Conclusions}

The data from Experiments 5-1, 5-2, and 5-3 suggest that male Papilio dardanus mate at random when naïve, but subsequently have an increased probability of mating with a female of the same morph with which they already have experience. Experiment 5-2 suggested that the probability of a male choosing the same morph on his second mating was around 0.75 , but as this figure is based on only 8 recorded second matings (when the male was given the choice of all three morphs) it can only be taken as a rough estimate. Since Cook et al. (1994) carried out mate choice experiments on wild males, some of these are likely to have been experienced, and to have mated previously. By comparing the results of Experiments 5-1 and 5-2 with the results of Cook et al. it might be possible to see to what extent the two sets of data are in accordance, and possibly estimate such unknown parameters as the number of times which a male mates in the wild (which will be valuable data for the construction of a mathematical model of the population in Chapter 7). 
The choice tests of Cook et al. were all performed as pairwise presentations. Either the data from these individual experiments will be additive (i.e. the probabilities of males choosing $A$ over $B$ and $B$ over $C$ in two pairwise tests will predict the probability of males choosing $A$ over $C$ in a further pairwise test), or the different pairings will affect the preferences of the males in a more complicated way (so that the probability of males choosing A over B and B over C will not predict the probability of males choosing A over $\mathrm{C}$ ). By analysing the data from Cook et al. it is possible to assess which of these two is the case, and it appears that the data can be taken to be additive, reflecting the overall preferences of the males (see Appendix 5 for the analysis). The preferences of the males (again see Appendix 5 for the calculations) are similar to the percentage of each female morph reported by Cook et al. from the area in which they were working (see Table 5-10).

\begin{tabular}{|c|c|c|}
\hline Morph & \% in population & \% preference by males \\
\hline hippocoonides & 80 & 63.16 \\
\hline trimeni & 16 & 26.32 \\
\hline lamborni & 4 & 10.52 \\
\hline
\end{tabular}

Table 5-10 Summary of the proportions of the morphs and the males' preferences as reported by Cook et al. (1994) from Pemba

If it is assumed (from the results of Experiment 5-1) that naïve males in the population mate at random, then it is possible to calculate from each of the percentage preferences recorded by Cook et al. the proportion of males which would be expected to have mated once already, using the probability that they choose the same morph on their second mating recorded in Experiment 5-2 (see Appendix 5 for these calculations).

Such a speculative analysis of the data from Cook et al. gives the unlikely result that around $97 \%$ of the males had already mated. This is a very high figure, and it seems very possible that the probability of males choosing the same morph twice, calculated from the results of Experiment 5-2, is inaccurate as it is based only on 8 data points. However, it is possible to calculate both this and the proportion of males who had already mated solely from the data of Cook et al. as three equations can be formed (one for each morph) and solved simultaneously.

By forming these three equations and plotting a surface of the error between the various predicted preferences for each morph (as calculated by changing both the proportion of males who had previously mated and the probability of them choosing the same morph twice in the equations) and the actual preferences calculated from Cook et al. previously it is possible to represent the error between the predicted and measured preferences as both the rate of mating twice and the probability of mating with the same morph twice vary. This is done in Appendix 5, and the minimum point on the surface (reflecting the best fit of the model to the observed values) is when both the proportion of the males who had mated twice and the probability that a male chooses the same morph on both matings is 0.84 . 
This high proportion for the probability of choosing the same morph twice is credible, as the value of 0.75 observed in Experiment 5-2 is based only on 8 data points and therefore has a very high margin of error. However, such a high proportion of second mating seems very unlikely to be accurate, and therefore one of several things may be occurring. Firstly, it is possible that the males on the island of Pemba do not mate at random on their first mating, as the males in Experiment 5-1 did, although it would be difficult to explain such a racial difference. Secondly it is possible that the results of Cook et al. do not simply reflect the underlying preferences of the males, and are affected by the pairwise method employed. Thirdly, the experimental design could be affected by the fact that the subjects are self-selecting. This could mean that only mated males, which have already had experience in recognising a mate, are attracted to the experimental apparatus. This would also explain why naïve males tested in the experimental flight cage did not respond to pinned-out dead females or models.

\section{Acknowledgements}

I would like to thank Phil Taylor for help with the flight cage, and John Calvert of Stratford Butterfly Farm for supplying the butterflies.

\section{References}

Aberle, D.F., Bronfenbrenner, J., Hess, E.H., Miller, D.R., Schneider, D.M. \& Spuhler, J.N. 1963. The incest taboo and the mating patterns of animals. American Anthropologist 65, 153-165.

Allen, G.R. \& Simmons, L.W. 1996. Coercive mating, fluctuating asymmetry and male mating success in the dung fly Sepsis cynipsea. Anim. Behav. 52, 737-741.

Bakker, T.M. \& Milinski, M. 1991. Sequential female choice and the previous male effect in sticklebacks. Behav. Ecol. Sociobiol. 29, 205-210.

Barlow, G.W., Francis, R.C., Baumgartner, J.V. 1990. Do the colors of parents, companions and self influence assortative mating in the polychromatic Midas Cichlid. Anim. Behav. 40, 713-722.

Belt, T. 1874. The naturalist in Nicaragua. Murray, London.

Breden, F., Novinger, D., \& Schubert, A. 1995. The effect of experience on mate choice in the Trinidad guppy Poecilia reticulata. Environmental Biology of Fishes 42, 323-328.

Brown, L. 1981. Patterns of female choice in mottled sculpins (Cottidae, teleleostei). Anim. Behav. 29, 375-382.

Burley, N \& Moran, N. 1979. The significance of age and reproductive experience in the mate preferences of feral pigeons, Columba livia. Anim. Behav. 27, 686-698.

Burns, J.M. 1968. Mating frequency in natural populations of skippers and butterflies as determined by spermatophore counts. Proc. Nat. Acad. Sci. 61, 852-859. 
Chai, P. \& Srygley, R.B. 1990. Predation and the flight, morphology and temperature of Neotropical rain-forest butterflies. Am. Nat. 135, 748-765.

Collins, S.A. 1995. The effect of recent experience on female choice in zebra finches. Anim. Behav. 49, 479-486.

Cook, D.F. 1992. The effect of male size on receptivity in female Lucilia cuprina (Diptera:

Calliphoridae). J. Insect Behav. 5, 365-374.

Cook, S.E., Vernon, J.G., Bateson, M., Guilford, T. 1994. Mate choice in the polymorphic African swallowtail butterfly, Papilio dardanus: male-like females may avoid sexual harassment. Anim.

Behav. 47: 389-397.

Cooke, F. \& Davies, J.C. 1983. Assortative mating, mate choice and reproductive fitness in Snow Geese. In Mate Choice (ed. Bateson, P.), Cambridge University Press, Cambridge. p279-298.

Clarke, C.A., Gordon, I.J., Vane-Wright, R.I., Smith, C.R. 1991. Phylogenetic relationships of three African swallowtail butterflies, Papilio dardanus, $P$. phorcas and P. constantinus: new data from hybrids (Lepidoptera: Papilionidae). Systematic Entomology 16, 257-273.

Clarke, C.A. \& Sheppard, P.M. 1959. The genetics of Papilio dardanus, Brown. I race cenea from South Africa. Genetics 44: 1347-1358.

Clarke, C.A. \& Sheppard, P.M. 1960a. The genetics of Papilio dardanus, Brown. II races dardanus, polytrophus, meseres and tibullus. Genetics 45: 439-457.

Clarke, C.A. \& Sheppard, P.M. 1960b. The genetics of Papilio dardanus, Brown. III race antinorii from Abyssinia and race meriones from Madagascar. Genetics 45: 683-698.

Clarke, C.A. \& Sheppard, P.M. 1962. The genetics of Papilio dardanus, Brown. IV data on race ochracea, race flavicornis, and further information on races polytrophus and dardanus. Genetics 47 : 909-920.

Clarke, C.A. \& Sheppard, P.M. 1963. Interactions between major genes and polygenes in the determination of mimetic patterns of Papilio dardanus. Evolution 17, 404-413.

Clarke, C.A., Gordon, I.J., Vane-Wright, R.I., Smith, C.R. 1991. Phylogenetic relationships of three African swallowtail butterflies, Papilio dardanus, $P$. phorcas and $P$ constantinus: new data from hybrids (Lepidoptera: Papilionidae). Systematic Entomology 16, 257-273.

Clutton-Brock, T.H., Hiraiwa-Hasegawa, M., \& Robertson,'A. 1989. Mate choice on fallow deer leks. Nature 340, 463-465.

Cook, D.F. 1995. Influence of previus mating experience on future mating success in male Lucilia cuprina (Diptera, Calliphoridae). J. Insect Behav. 8, 207-217.

Cook, S.E., Vernon, J.G., Bateson, M., Guilford, T. 1994. Mate choice in the polymorphic African swallowtail butterfly, Papilio dardanus: male-like females may avoid sexual harassment. Anim.

Behav. 47: 389-397.

Dale, S., Amundsen, T., Lifjeld, J.T. \& Slagsvold, T. 1990. Mate sampling behaviours of female pied flycatchers - evidence for active mate choice. Behav. Ecol. Sociobiol. 27, 87-91.

Dawkins, M.S. \& Guilford, T. 1995. An exaggerated preference for simple neural network models of signal evolution? Proc. R. Soc. Lond. B. 261, 357-360. 
D'Udine, B. \& Alleva, E. 1983. Early experience and sexual preferences in rodents. In Mate Choice (ed Bateson, P.) Cambridge University Press, Cambridge. p31 1-327.

Dufour, K.W. \& Weatherhead, P.J. 1998. Reproductive consequences of bilateral asymmetry for individual male red-winged blackbirds. Behav. Ecol. 9, 232-242.

Enquist, M. \& Arak, A. 1994. Symmetry, beauty, and evolution. Nature 372, 169-172.

Ferno, A. \& Sjölander, S. 1973. Some imprinting experiments on sexual preferences for colour variants in the platyfish (Xiphophorus maculatus). Z. Tierpsychol. 33, 417-423.

Ford, E.B. 1936. The genetics of Papilio dardanus Brown (Lep.) Trans. R. Ent. Soc. London 85, 435-465.

Giurfa, M., Eichmann, B. \& Menzel, R. 1996. Symmetry perception in an insect. Nature 382, 458461.

Harvey, I.F. \& Walsh, K.J. 1993. Fluctuating asymmetry and lifetime mating success are correlated in males of the damselfly Coenagrion puella (Odonata, Coenagrionidae). Ecol. Entomol. 18 (3), 198202.

Haskins, C.P. \& Haskins, E.F. 1950. Factors governing sexual selection as an isolating mechanism in the poeciliid fish Lebistes reticulatus. Proc. Nat. Acad. Sci. USA 36, 464-476.

Hespenheide, H.A. 1975. Reverse sex-limited mimicry in a beetle. Evolution 29, 780-783.

Immelmann, K. 1985. Sexual imprinting in zebra finches: mechanisms and biological significance. Proc. Int. Ornithol. Congr. 18, 156-172.

Johnstone, R.A. 1994. Female preference for symmetrical males as a by-product of selection for mate recognition. Nature 372, 172-175.

Kop, P.A.M. \& Heuts, B.A. 1973. An experiment on sibling imprinting in the jewel fish (Hemichromis bimaculatus). Rev. Comp. Animal 7, 63-76.

Liley, N.R. 1966. Ethological isolating mechanisms in four sympatric species of poeciliid fishes. Behav. Suppl. 13, 1-197.

Magnus, D.B.E. 1958. Experimentelle Untersuchungen zur Bionomie und Ethologie des Kaisermantels. Z. Tierpsychol. 15, 397-426.

McLachlan, A. \& Cant, M. 1995. Small males are more symmetrical - mating success in the midge Chironomus plumosus. Anim. Behav. 50, 841-881.

Manning, J.T. \& Hartley, M.A. 1991. Symmetry and ornamentation are correlated in the peacock's train. Anim. Behav. 42, 1020-1021.

Markow, T.A. \& Ricker, J.P. 1992. Male size, developmental stability, and mating success in natural populations of three Drosophila species. Heredity 69, 122-127.

Møller, A.P. 1992. Female swallow preference for symmetrical male ornaments. Nature 357, 238240.

Møller, A.P. \& Sorci, G. 1998. Insect preference for symmetrical artificial flowers. Oecologia 114, 37-42. 
Møller, A.P. \& Thornhill, R. 1998. Bilateral symmetry and sexual selection: a meta-analysis. Am. Nat. 151, 174-192.

Møller, A.P. \& Zamora-Muñoz, C. 1997. Antennal asymmetry and sexual selection in a cerambycid beetle. Anim. Behav. 54, 1509-1515.

Oakes, E.J. \& Barnard, P. 1994. Fluctuating asymmetry and mate choice in paradise whydahs Vidua paradisaea - an experimental manipulation. Anim. Behav. 48, 937-943.

Parsons, P.A. 1990. Fluctuating asymmetry - an epigenetic measure of stress. Biol. Rev. 65, 131-145.

Pomiankowski, A. 1990. How to find the top male. Nature 347, 616-617.

Radesäter, T. \& Halldórsdóttir, H. 1993. Fluctuating asymmetry and forceps size in earwigs, Forficula auricularia. Anim. Behav. 45, 626-628.

Rutowski, R.L. 1985. Evidence for mate choice in a sulphur butterfly (Colias eurytheme). $Z$. Tierpsychol. 70, 103-114.

Simmons, L.W. 1995. Correlates of male quality in the field cricket Gryllus campestris L.: age, size, and symmetry determine pairing success in field populations. Behav. Ecol. 6, 376-81.

Silberglied, R.E. 1984. Visual communication and sexual selection among butterflies. In The biology of butterflies (ed. Vane-Wright, R.I. \& Ackery, P.R.). Acadmeic Press, London. p207-223.

Sjolander, S. \& Ferno, A. 1973. Sexual imprinting on another species in a cichlid fish, Haplochromis burtoni. Rev. Comport. Anim. 7, 77-81.

Smith, D.A.S. 1984. Mate selection in butterflies: competition, coyness, choice and chauvenism. In The Biology of Butterflies (ed. Vane Wright, R.I. \& Ackery, P.R.) Princeton University Press, USA, 225-244.

Stride, G.O. 1958. Further studies on the courtship behaviour of African mimetic butterflies. Anim. Behav. 6, 224-230.

Svärd, L. \& Wicklund, C. 1986. Different ejaculate delivery strategies in first versus subsequent matings in the swallowtail butterfly, Papilio machaon L. Behav. Ecol. Sociobiol. 18, 325-330.

Swaddle, J.P. \& Cuthill, I.C. 1994. Selection for symmetrical males by female zebra finches. Nature 367, $165-6$

Swihart, C.A. 1971. Colour discrimination by the butterfly, Heliconius charitonius L.. Anim Behav. 19, 156-164.

Thornhill, R. 1992. Fluctuating asymmetry and mating success in the Japanese scorpionfly, Panorpa japonica. Anim. Behav. 44, 867-879.

Thornhill, R. \& Gangestad, S.W. 1993. Human facial beauty: averageness, symmetry, and parasite resistance. Human Nature 4, 237-269.

Trimen, R. 1869. On some remarkable mimetic analogies among African butterflies. Transactions of the Linnean Society of London 26, 497-522.

Turner, J.R.G. 1978. Why male butterflies are non-mimetic: natural selection, sexual selection, group selection, modification and sieving. Biol. J. Linn. Soc. 10, 385-432. 
Vane-Wright, R.I. \& Smith, C.R. 1991. Phylogenetic relationships of three African swallowtail butterflies, Papilio dardanus, $P$. phorcas, and P. constantinus: a cladistic analysis (Lepidoptera: Papilionidae). Systematic Entomology 16: 275-291.

Van Valen, L. 1962. A study of fluctuating asymmetry. Evolution 36, 768-776.

Vos, D.R. 1995. The role of sexual imprinting for sex recognition in zebra finches - a difference between males and females. Anim. Behav. 50, 645-653. 


\section{Chapter Six}

\section{The Palatability of Papilio dardanus}

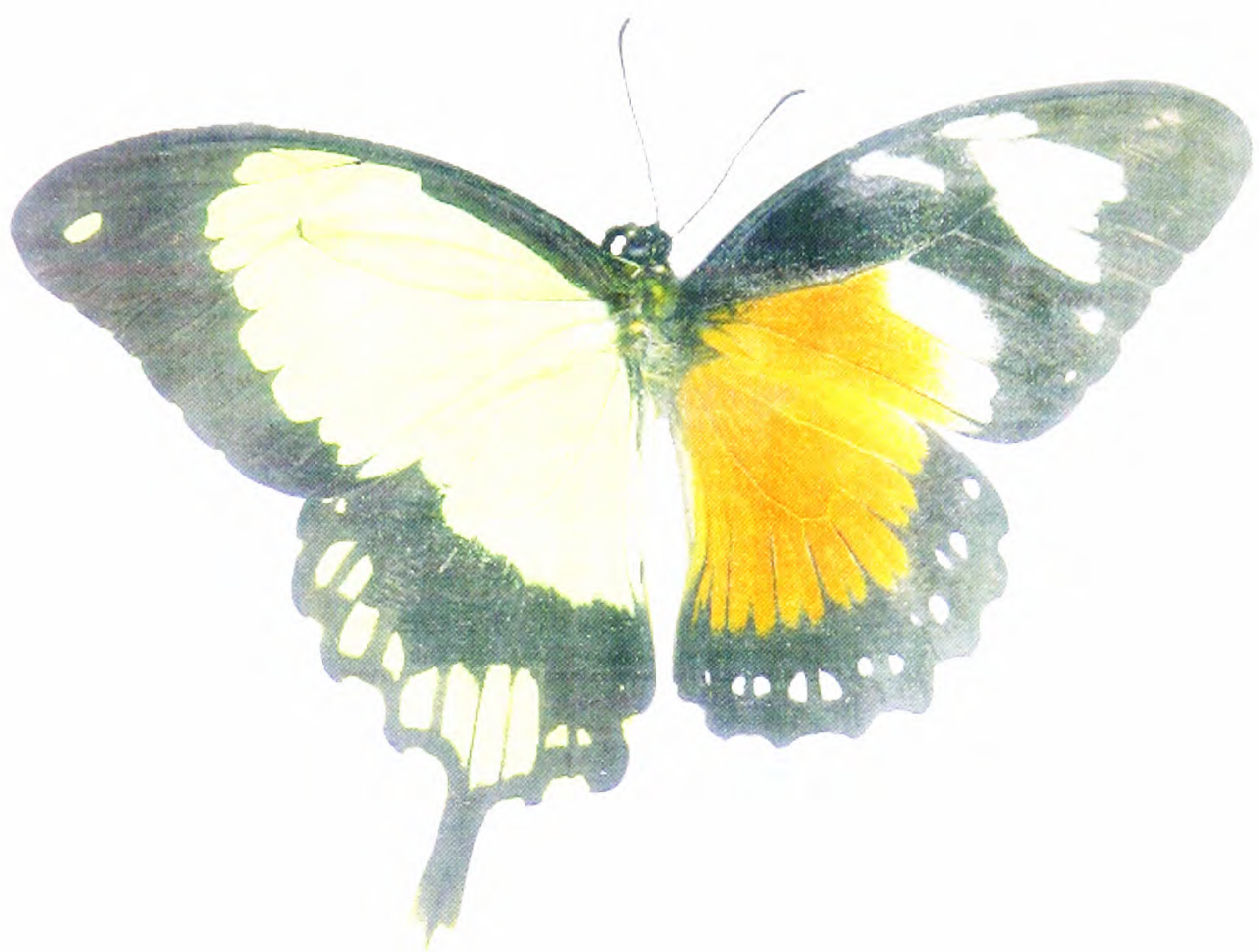

Figure 6-1 A gynandromorph of Papilio dardanus showing the difference in morphology of males and females, used to predict palatability in Experiment 6-6

"I personally have made a habit, which I recommend to other naturalists, of eating specimens of every species which I study'

E.B. Ford (1955), p103 


\section{Introduction}

The theory of mimicry requires that some individuals are less profitable to predators than others. The actual palatability of a species, however, is not easy to ascertain as it depends on many factors. Firstly, different predators may have different tastes. For example, some bird species (Pheucticus melanocephalus and Icterus galbula) and mice (Peromyscus melanotis) have been shown to eat overwintering Monarch butterflies (Danaus plexippus), being resistant to their poisons (Fink \& Brower, 1981, Glendinning \& Brower, 1990). The palatability of a particular prey item to the predator also depends on the psychological state of the predator - for example how hungry it is (Swynnerton, published posthumously by Carpenter, 1941). Secondly, individuals within a species may have differing palatabilities, for example Monarch butterflies which have fed on milkweed species, such as Asclepias curassavica, which contain cardiac glycosides are able to sequester the chemicals (calactin, calotropin and calotoxin), whilst those which have fed on species lacking these emetic chemicals, such as Gonolobus rostratus are completely palatable to bird predators (Brower, 1969). This results in a 'palatability spectrum' (Brower et al., 1968) of individuals within and between species for a particular predator. The point at which the predator distinguishes 'unpalatable' from 'palatable' depends on the state of that individual.

Given these complications, experiments to test the relative palatabilities of different prey items are difficult to plan and carry out. The simple chemical identification of a compound in a prey item which can be shown to be unpalatable to a major predator may not be evidence that the predator would avoid the prey item under natural conditions. The most naturalistic experiments involve releasing captured butterflies to wild birds feeding from a perch (e.g. Swynnerton, 1915, 1919; Carpenter, 1941), but this only tests the perceived palatability of the prey to the predator, which may be mistaking mimetic species for their models, and also needs to be carried out throughout the year to measure the average palatability (e.g. Srygley \& Kingsolver, 1998). In order to overcome this problem, the coloration of the prey could be disguised, and naïve predators used.

Papilio dardanus is a classic example of Batesian mimicry. The palatable females mimic unpalatable species in order to gain protection from predators. However, in none of the experiments by Swynnerton (Carpenter 1941) in which he released wild-caught butterflies to wild birds (mostly the bee eater Merops merops) was a male Papilio dardanus eaten by the birds, although two were caught and immediately released. This suggests that the butterflies may have at least appeared to be distasteful to the birds. In addition, the wings of the species smell strongly of pyrazines, chemicals thought to act as a chemical signal of distastefulness in combination with bright warning colours, such as those found in Papilio dardanus (Rowe \& Guilford, 1996), and which are difficult to isolate and identify chemically, requiring gas chromatography and mass spectrometry (Rothschild et al., 1984). 
There is much debate about the reason why a male-like andromorph is found in some populations of Papilio dardanus (such as the tailed yellow females in races antinorii, meriones, and humbloti, and the tailless morph trimeni in race tibullus). Many argue that the male-like female is ancestral (Poulton, 1924; Ford, 1936; Clarke \& Sheppard, 1963; Turner, 1963; O'Donald \& Barrett, 1973; Clarke et al., 1985), but do not explain how the morph is maintained in a population which includes Batesian mimics which must be at an advantage in avoiding predation. Where the race is entirely monomorphic, such as races meriones and humbloti, it can be argued that the mimetic morphs have simply not evolved (possibly due to a lower degree of predation as these morphs are confined to islands where bird fauna is impoverished) (Trimen, 1868). Others argue that the male-like females may be derived (van Bemmelen, 1922; Bernardi, 1963; Vane-Wright \& Smith, 1991, Vane-Wright et al., in press), possibly mimicking the males in order to avoid sexual harassment (Cook et al., 1994) although this latter hypothesis seems less likely in the light of the observations and results of Chapter 5. The fact that their genes are dominant over those of most other female morphs (excluding lamborni) in the populations in which they exist suggests that they may have a more recent origin (Sheppard, 1967), although, as discussed in the conclusions to Chapter 3, the tendency for new genes to become dominant has been shown mathematically to be true only under certain conditions (Charlesworth \& Charlesworth, 1976). In Chapter 5 it was shown that the males appear to have no morph preferences when mating for the first time but subsequently show a preference for the morph with which they first mated, and therefore that the andromorphic females were unlikely to be at any advantage through sexual selection. Whether they are merely the ancestral morph, maintained in the population, or whether they are a recently evolved male-mimic, there must be some selective pressure resulting in them being present in the polymorphic, mimetic populations.

The presence of a pyrazine odour on the wings of Papilio dardanus is likely to reinforce the warning effect of the coloration on the wings of the mimetic morphs (Rowe \& Guilford, 1996), but all individuals carry the same odour, and so the bright yellow of the males and andromorphs may have the same warning effect in association with the pyrazine odour. Rowe \& Guilford also found, however, that the signal loses its significance if the warning odour and coloration is not backed up by unpalatability. If the species is in fact slightly unpalatable then the yellow coloration and pyrazine odour would act as an honest warning. The effect seen by Swynnerton (Carpenter, 1941) of two birds releasing male Papilio dardanus once they had been caught does accord with the hypothesis that they were deterred from eating the butterflies because of the pyrazine odour they emitted when caught. It is possible therefore that the species is not, in fact, completely palatable to its major predators. The existence of a palatability spectrum (Brower et al., 1968) means that many cases of mimicry might be expected to fall between the standard definitions of Batesian and Mullerian mimicry, and the population dynamics and evolutionary pressures could be very different (Speed, 1993). The males could therefore be honest signallers of unpalatability, and it is conceivable that the mimetic female morphs could still benefit from mimicking more strongly unpalatable species, or that the palatability 
of the species has decreased recently - after the evolution of the female Batesian mimics. If the andromorphs are recently evolved, it is possible that their evolution is due to this decrease in the palatability of the species, and that they are intraspecific Müllerian mimics. If they are in fact the ancestral form, then their presence in coincidence with the other mimetic morphs would be more difficult to explain, in the absence of other balancing factors.

In order to test the hypothesis that Papilio dardanus is not entirely palatable to predators, experiments were planned in which birds were fed Papilio dardanus, one of its unpalatable models, and palatable butterflies for comparison.

In addition, an indirect measure of the palatability of the species was used. It has been shown (Chai \& Srygley, 1990; Srygley \& Dudley, 1993) that certain morphological traits in butterflies are associated with varying degrees of palatability (as they affect the flight and hence escape capabilities of the insect). Therefore morphological data was collected in accordance with Srygley \& Dudley (1993) to assess whether or not Papilio dardanus has the physical structure of an unpalatable butterfly.

\section{$\underline{\text { Aim }}$}

The aim of this chapter is to assess the palatability of Papilio dardanus compared with a model for one of the female morphs, Amauris niavius (model for hippocoonides) or Danaus chrysippus (model for lamborni/trophonius). This data should clarify the evolutionary pressures on the species, and be important for the formation of a mathematical model of the populations in Chapter 7.

\section{Experiment 6-1 Pilot test to determine the suitability of hen chicks for palatability experiments}

\section{Introduction}

Hen chicks (Gallus gallus domesticus) have been shown to be discriminating artificial predators, demonstrating aversion to typical unpalatable substances such as quinine and mustard (Gittleman \& Harvey, 1980). It was therefore decided to carry out an initial pilot test to determine whether or not they showed aversion to crumbs flavoured with known unpalatable butterflies, or to those flavoured with Papilio dardanus in the same way as their aversion was tested to quinine and mustard by Rowe \& Guilford, 1996. If aversion were to be displayed, then further experiments could 
be carried out to test whether or not they failed to show aversion to known palatable butterflies or whether the aversion was more generally to crumbs flavoured with butterflies.

Dried danaine butterflies have been shown to be highly unpalatable to birds (Dixon et al., 1978), and so refrigerated, desiccated specimens were used to flavour the crumbs.

\section{Aim}

The aim of this experiment is to determine whether or not hen chicks (Gallus gallus domesticus) can be used to assess the palatability of Papilio dardanus compared with Amauris niavius.

\section{Materials and Method}

The bodies of two hippocoonides females of Papilio dardanus which had been raised on their natural foodplant (Citrus spp.) in Africa were dessicated over a period of months, and ground into a fine powder. Chick-starter crumbs (Allen \& Page, Norfolk Feeds) were damped with a water spray, and then coated in the powder and left under a hot lamp until dry.

The same procedure was carried out with the wings of two hippocoonides females, and the bodies and wings of two Amauris niavius.

This resulted in four different treatments of crumbs:

1) hippocoonides female bodies

2) hippocoonides female wings

3) Amauris niavius female bodies

4) Amauris niavius female wings

Three day old hen chicks (Gallus gallus domesticus) from a commercial hatchery which had previously been fed on plain chick-starter crumbs (Allen \& Page, Norfolk Feeds), were used in the assessment. The chicks were food deprived for 2-3 hours before the assessments. Two chicks were placed in a circular arena containing a handful of one of the four differently treated groups of crumbs in the centre. Their behaviour was noted, and the time they took to eat the crumbs was recorded. They were then returned to the cage, and their behaviour noted for 30 minutes. This was repeated for each of the four treatments, using different chicks each time. 
A few hours later, the chicks were again food-deprived, and put in the arena in pairs. This time they were given the crumbled body and wings of either a desiccated Amauris niavius or a hippocoonides female. Again their behaviour was observed during their time in the arena and for 30 minutes after being returned to the cage. This experiment was repeated twice more the following day with the same chicks receiving the same treatments to ascertain whether or not avoidance had been learnt.

\section{Results}

In all four cases, the chicks readily ate all the crumbs presented, and showed no ill effects (such as beak-wiping, head shaking, and large bouts of drinking - Brower et al., 1967). When presented with crumbled butterflies, the chicks ate these just as readily, finishing them within ten minutes. Again, they showed no ill-effects at all. When the experiment was repeated the following day, the chicks appeared to show no aversion to either butterfly, and ate them equally fast with all crumbs being eaten within 10 minutes.

\section{Discussion}

Neither desiccated Amauris niavius nor Papilio dardanus appeared to be distasteful to the young chicks. Therefore they are not suitably discriminative predators to be used to ascertain the relative palatability of Papilio dardanus. Although fresh butterflies may be more unpalatable to the birds, it was not possible to acquire a large number of fresh distasteful butterflies.

\section{Experiment 6-2 Pilot test to determine the suitability of starlings (Sturnus vulgaris) for palatability experiments}

\section{Introduction}

Starlings (Sturnus vulgaris) have been used successfully in palatability experiments (Brower, 1960). They were therefore tested in the same way as the hen chicks in Experiment 6-1 to determine whether or not they showed any discrimination between known palatable and unpalatable butterflies.

\section{Aims}

The aim of this experiment is to determine whether or not starlings (Sturnus vulgaris) can be used to determine the relative palatabilities of Papilio dardanus and one of its models (Amauris niavius). 


\section{Materials and Methods}

This experiment used individually caged wild-caught starlings (Sturnus vulgaris) kept in temperature controlled rooms (three cages per room). The starlings were usually fed on turkey crumbs (Allen \& Page, Norfolk Feeds). Throughout the experiments the birds were given free access to water.

Initially three starlings were food-deprived for 18 hours and then given $100 \mathrm{~g}$ turkey crumbs which had been mixed with ground up hippocoonides females of Papilio dardanus (approximately 2 whole butterflies in $100 \mathrm{~g}$ crumbs) in the method described in Experiment 6-1. Their behaviour was observed for an hour through one-way glass. Secondly, six starlings were food deprived for 17 hours. They were then each given $1 \mathrm{lg}$ of turkey crumbs - three were plain crumbs and the other three were flavoured with one whole powdered butterfly, one each of Amauris niavius, Papilio dardanus (hippocoonides female), and Aglais urticae (a British nymphalid butterfly, for which there is no evidence of unpalatability). After 6 hours, the amount of food each had eaten and water they had drunk was measured. Finally, two birds were food-deprived for 18 hours and were then given three whole butterflies, roughly crumbled (either Papilio dardanus cenea females or Amauris niavius). Their behaviour was monitored for several hours.

\section{Results}

The first three starlings showed no symptoms of distress (such as excessive drinking, beak wiping and head shaking) when fed turkey crumbs mixed with powdered Papilio dardanus. They did show some beak-wiping, and drank regularly, but this was also observed when they were fed plain turkey crumbs.

The birds which were given exactly $11 \mathrm{~g}$ of treated crumbs ate and drank the following amounts in 6 hours (the amount drunk was assessed by measuring the change in level of the water in the water bottles, in centimeters):

\begin{tabular}{|l|c|c|}
\hline \multicolumn{1}{|c|}{ Starling fed: } & Amount eaten & Amount drunk \\
\hline plain crumbs & $2.1 \mathrm{~g}$ & 1.7 units \\
\hline plain crumbs & $7 \mathrm{~g}$ & 1.7 units \\
\hline plain crumbs & $1 \mathrm{~g}$ & 5.5 units \\
\hline Amauris niavius crumbs & $0.05 \mathrm{~g}$ & 1.8 units \\
\hline Papilio dardanus crumbs & $5 \mathrm{~g}$ & 1.1 units \\
\hline Aglais urticae crumbs & $6.72 \mathrm{~g}$ & 2 units \\
\hline
\end{tabular}

Table 6-1 The amounts of treated and untreated crumbs eaten, and amounts of water drunk, by 6 individual starlings 
The starling which was given three crumbled Papilio dardanus ate them (including the wings) without signs of particular distress. However, the bird which was given three crumbled Amauris niavius butterflies ate only one piece, then showed prolonged beak-wiping, and did not eat any more of the butterflies.

\section{Discussion}

This small pilot test suggests that the starlings may find turkey crumbs mixed with powdered Amauris niavius unpalatable. It also shows that the starlings will eat 'undisguised' butterflies which have simply been crumbled up, and can thus be induced to take much more concentrated doses of the butterflies (as would a predator in the wild). The reaction of the bird which was given a whole Amauris niavius also suggests that they might find Amauris niavius distasteful. This evidence, although inconclusive, indicates that further experiments should be carried out using starlings as discriminative predators in order to determine whether or not they can be used to place Papilio dardanus on a palatability spectrum ranging from Amauris niavius or Danaus chrysippus to known completely palatable butterflies.

\section{Experiment 6-3 A discrimination test using dried butterflies and starlings (Sturnus vulgaris)}

\section{Introduction}

The pilot test, Experiment 6-2 indicated that starlings (Sturnus vulgaris) may find Amauris niavius distasteful. However, it was unfortunately not possible to obtain further supplies of Amauris niavius. Therefore, Danaus chrysippus (the putative model for the trophonius/lamborni morph) was used as an unpalatable butterfly instead. This experiment is designed to test whether or not the starlings can show a great enough degree of discrimination to be able to place Papilio dardanus in the palatability spectrum between Danaus chrysippus, a known unpalatable butterfly, and Charaxes species, shown to be highly palatable by Swynnerton (Carpenter, 1941).

The experiment was designed to test not only their initial reaction to ingesting a particular butterfly, but to then offer the birds a choice to determine whether or not they avoided the species they had just been exposed to, or preferred it to the new species which they had just been introduced to. They were then left with just those two species and no other food, to measure how much of each species they ate as time went on and they became hungrier. 


\section{Aim}

The aim of this experiment is to determine the relative palatabilities of Papilio dardanus, one of its distasteful models, Danaus chrysippus, and palatable Charaxes butterflies using food deprived starlings (Sturnus vulgaris) as discriminative predators.

\section{Materials and Methods}

8 starlings, which had not been used in palatability studies previously, were food deprived for 18 hours, but given open access to water throughout. The starlings were allocated to one of three groups, and each was then given three individual butterflies (which had been refrigerated post mortem for up to 6 months), broken into three pieces and with the wings crumbled into pieces:

Group A (2 birds) - three Charaxes butterflies each

Group B (2 birds) - three Danaus chrysippus each

Group C (4 birds) - three Papilio dardanus females each (one hippocoonides, one lamborni and one cenea for each bird)

The behaviour of each starling was observed for one hour after feeding. At the end of this time, any remaining butterfly material was assessed and the number of pieces eaten by each bird recorded (the crumbled wings were counted as one piece, making the number of pieces of each species 10). The 2 (or 4 ) birds in each group were then allocated to a sub group, and immediately given a choice of each of two food sources, each prepared in the same way as before:

Group A1 - choice between Charaxes and Danaus chrysippus

Group A2 - choice between Charaxes and Papilio dardanus

Group B 1 - choice between Danaus chrysippus and Charaxes

Group B2 - choice between Danaus chrysippus and Papilio dardanus

Group Cl - choice between Papilio dardanus and Charaxes

Group C2 - choice between Papilio dardanus and Danaus chrysippus

The behaviour of each starling was again observed for one hour after feeding, and at the end of this time remaining pieces of butterfly were counted and the amount of each species eaten by each bird recorded as before. The birds were then left with the same choice for 4 further hours, and at the end of this period (a total of 24 hours) any remaining amounts were again counted. 


\section{Results}

\begin{tabular}{|c|c|c|c|c|c|c|}
\hline Bird & \multicolumn{2}{|c|}{ First presentation } & \multicolumn{4}{c|}{ Second presentation } \\
& Species & Amount eaten & Species 1 & Amount eaten & Species 2 & Amount eaten \\
\hline 1(A2) & Charaxes & 0 & Charaxes & 0 & Papilio & 0 \\
\hline 2(A1) & Charaxes & 8 & Charaxes & 10 & Danaus & 10 \\
\hline 3(C1) & Papilio & 10 & Papilio & 10 & Danaus & 6 \\
\hline $4(\mathrm{C} 2)$ & Papilio & 10 & Papilio & 5 & Charaxes & 7 \\
\hline 5(C1) & Papilio & 2 & Papilio & 10 & Danaus & 10 \\
\hline $6(\mathrm{C} 2)$ & Papilio & 0 & Papilio & 10 & Charaxes & 10 \\
\hline 7(B2) & Danaus & 0 & Danaus & 10 & Papilio & 10 \\
\hline 8(B1) & Danaus & 0 & Danaus & 10 & Charaxes & 10 \\
\hline
\end{tabular}

Table 6-2 The amounts eaten of different butterflies by 8 starlings

\section{Discussion}

The starlings appeared to be indiscriminate in what they ate, most eating everything they were presented with. This could either be because the dried specimens did not differ in palatability to the birds, or because the hunger levels of the birds were so high as to cause them not to distinguish between the palatabilities of what they were presented with. As mentioned previously, large numbers of fresh specimens of the butterflies (other than Papilio dardanus) were very difficult to obtain, and so the experiment was repeated with a shorter food deprivation time in order to decrease the hunger levels of the birds.

\section{Experiment 6-4 A further discrimination test using dried butterflies and starlings (Sturnus vulgaris)}

\section{Introduction}

In Experiment 6-3, the starlings failed to discriminate between known palatable (Charaxes) and known unpalatable (Danaus chrysippus) butterflies. This could be due to the dried butterfly specimens losing their unpalatability. However, it was not possible to obtain a large number of fresh specimens of a known unpalatable species. An alternative is that the hunger levels of the starlings were too high to allow them to discriminate between the species. Therefore, another test was carried out, repeating the general method of Experiment 6-4 but decreasing the food deprivation period of the experimental birds. 


\section{Aim}

The aim of this experiment is to determine whether or not starlings with a lower level of hunger will discriminate between known palatable (Charaxes) and unpalatable (Danaus chrysippus) butterflies, and also to test the relative palatability of Papilio dardanus with respect to these two extremes.

\section{Method and Materials}

The same basic method of Experiment 6-3 was used. 6 starlings, not previously used in palatability experiments, were food deprived for 5 hours, but given free access to water during this period. The starlings were then allocated to one of three groups, and each was then given three individual butterflies (which had been refrigerated post mortem for up to one year), each broken into three pieces and with the wings crumbled into pieces:

Group A - three Charaxes butterflies each

Group B - three Danaus chrysippus each

Group C - three Papilio dardanus females each (one hippocoonides, one lamborni and one cenea)

The behaviour of each starling was observed for one hour after feeding. At the end of this time, any remaining butterfly pieces were counted and the amount eaten by each bird recorded (the crumbled wings were counted as one piece, giving each bird a score out of 10 for each butterfly species). The 2 birds in each group were then allocated to a sub group, and given a choice of each of two butterflies, prepared in the same way as in the first part of the experiment:

Group A1 - choice between Charaxes and Danaus chrysippus

Group A2 - choice between Charaxes and Papilio dardanus

Group B1 - choice between Danaus chrysippus and Charaxes

Group B2 - choice between Danaus chrysippus and Papilio dardanus

Group C1 - choice between Papilio dardanus and Charaxes

Group C2 - choice between Papilio dardanus and Danaus chrysippus

The behaviour of each starling was again observed for one hour after feeding, and at the end of this time the number of pieces of each butterfly remaining and the amount of each species eaten by each bird were recorded as before. The birds were then left with the same choice for 4 further hours, and at the end of this period (a total of 11 hours) any remaining pieces were again counted. 


\section{Results}

\begin{tabular}{|r|c|c|c|c|c|c|}
\hline Bird & \multicolumn{2}{|c|}{ First presentation } & \multicolumn{4}{c|}{ Second presentation } \\
& Species & Amount eaten & Species 1 & Amount eaten & Species 2 & Amount eaten \\
\hline 1(A1) & Charaxes & 0 & Charaxes & 0 & Danaus & 0 \\
\hline 2(A2) & Charaxes & 9 & Charaxes & 10 & Papilio & 10 \\
\hline $3(\mathrm{C} 2)$ & Papilio & 9 & Papilio & 0 & Danaus & 3 \\
\hline 4(C1) & Papilio & 6 & Papilio & 1 & Charaxes & 3 \\
\hline 5(B2) & Danaus & 10 & Danaus & 10 & Papilio & 10 \\
\hline 6(B1) & Danaus & 10 & Danaus & 10 & Charaxes & 9 \\
\hline
\end{tabular}

Table 6-3 The amounts eaten of different species of butterfly by six starlings

\section{Discussion}

As in Experiment 6-3, there appears to be no pattern to the feeding of the starlings. Some ate almost all that was presented to them, and others ate very little. The birds did not appear to avoid the unpalatable Danaus chrysippus. The observed difference in behaviour could be due to differing hunger levels in the individuals. With relatively short periods of food deprivation it is very difficult to ensure that all the birds are in the same state. However, even those that did not eat everything presented to them appeared not to avoid the Danaus chrysippus (e.g. bird 3) and hence it appears that the dried butterfly specimens have lost any unpalatability they may have had for the birds, or that the birds do not find the chemicals contained in Danaus chrysippus (thought to be cardenolides and pyrrolizidine alkaloids - Meinwald et al., 1974) distasteful. Swynnerton (1915, 1919) found some birds very sensitive to the compounds in Danaus chrysippus (one wood hoopoe, Phoeniculus purpureus, vomiting after eating a single Danaus chrysippus, and another after eating a single Amauris echeria - the model for the cenea morph), whilst others seemed completely insensitive (rollers, Coracias garrulus; yellow-billed hornbills, Tockus flavirostrus; crowned hornbills, Tockus alboterminatus; and babblers, Turdoides jardinei ate up to 50 danaines without vomitting). This could be due to cardenolide insensitivity (Brower, 1984) or due to the presence of cardenolide-free danaines in the population (Brower et al., 1975, 1978). The same explanations could be true for the starlings not finding the danaines unpalatable.

\section{Experiment 6-5 A discrimination test using fresh butterflies and starlings (Sturnus vulgaris)}

\section{Introduction}

Experiment 6-4 indicated that starlings (Sturnus vulgaris) did not find dried specimens of either Papilio dardanus or Danaus chrysippus unpalatable, but it was not possible to determine whether this was due to the dried nature of the butterflies, whether the birds were resistant to the 
effects of the cardenolides and pyrrolizidine alkaloids of Danaus chrysippus (Brower, 1984), or whether the individual butterflies were lacking in protection (Brower et al., 1975, 1978). It was possible to obtain a small number of fresh Danaus chrysippus from Malaysia, and so it was possible to design a small experiment to test which of these factors was causing the starlings to be indiscriminate.

$\operatorname{Aim}$

The aim of this experiment is to determine whether or not starlings (Sturnus vulgaris) find fresh Danaus chrysippus or Papilio dardanus distasteful.

\section{Method and Materials}

Experiment 6-4 illustrated how a short food deprivation period could result in different levels of hunger in different individuals, leading to very different degrees of discrimination. A small pilot test indicated that the starlings would eat small pieces of butterfly even without a period of food deprivation, and so the experiment was designed without deprivation, giving the starlings a choice between their normal dried turkey crumbs (Allen \& Page, Norfolk Feeds) and small pieces of butterfly.

Six starlings (the same birds as used in Experiment 6-4), fed normally on turkey crumbs (Allen \& Page, Norfolk Feeds) and given free access to water at all times were allocated randomly to one of two groups. The three birds in Group A were each given one freshly killed Papilio dardanus, the body divided into five pieces, in one half of a dual feeder, and a supply of their normal turkey crumbs in the other half. The birds in Group B were given one freshly killed Danaus chrysippus, divided in the same manner, in one half of the feeder, and a supply of turkey crumbs in the other half.

Both sets of birds were left for four hours, after which time the number of pieces of butterfly they had eaten was counted (recorded as a score out of 5). Any remaining pieces were then removed, and the birds left with just their normal food.

This procedure was then repeated twice more, at 24 hour intervals, with the groups remaining the same as on the previous day, and the number of pieces of the butterflies eaten by each starling on each day recorded. 


\section{Results}

The amounts eaten by each starling on each day are shown in Table 6-4.

\begin{tabular}{|c|c|c|c|c|}
\hline \multicolumn{2}{|c|}{ Bird number } & \multicolumn{3}{|c|}{ Number of pieces eaten } \\
\hline & & Day 1 & Day 2 & Day 3 \\
\hline \multirow{3}{*}{$\begin{array}{c}\text { Group A } \\
\text { (Papilio } \\
\text { dardanus) }\end{array}$} & 1 & 5 & 5 & 5 \\
\hline & 2 & 5 & 5 & 5 \\
\hline & 3 & 5 & 5 & 5 \\
\hline \multirow{3}{*}{$\begin{array}{c}\text { Group B } \\
\text { (Danaus } \\
\text { chrysippus) }\end{array}$} & 4 & 1 & 0 & 0 \\
\hline & 5 & 5 & 5 & 5 \\
\hline & 6 & 1 & 5 & 5 \\
\hline
\end{tabular}

Table 6-4 The number of pieces of either Papilio dardanus or Danaus chrysippus eaten by starlings on each of three consecutive days

\section{Discussion}

The small numbers of birds and butterflies used makes the results difficult to interpret.

However, the results suggest that the fresh Danaus chrysippus may not be quite as palatable as the fresh Papilio dardanus to the starlings although bird 4 in this experiment, which ate very little of the Danaus chrysippus is the same bird as Bird 1 in Experiment 6-4, which ate none of the butterflies presented (although it did eat mealworms presented in the same food hopper between experiments).

The Danaus chrysippus did not have any emetic effect on the starlings, and the birds did not appear to find them particularly distasteful (as they ate the butterflies without any food deprivation, and whilst there was other food available). It thus appears that either the starlings are not affected by the distasteful compounds in Danaus chrysippus or these individual butterflies come from a population which does not contain such compounds. It appears clear, however, that Papilio dardanus is highly palatable to the starlings (it has been eaten in every experiment, and in this experiment it was eaten in preference to the turkey crumbs).

\section{Experiment 6-6 Comparison of the morphology of Papilio dardanus and its models}

\section{Introduction}

It has been shown that some morphological traits, such as thoracic diameter, abdominal length, and the position of the centre of mass of the body can be used as predictors of palatability (Chai \& Srygley, 1990; Srygley \& Dudley, 1993, Srygley, 1994). This is due to the fact that palatable butterflies tend to allocate more resources to flight muscle in the thorax (increasing the thoracic diameter) enhancing flight speed and acceleration in case of attack by birds (Chai \& Srygley, 1990). 
They also have shorter abdomens, possibly in order to decrease the chance of being caught by the abdomen and to increase aerobatic ability (Chai \& Srygley, 1990). Furthermore, they tend to have a centre of mass positioned closer to the wing base than unpalatable butterflies, which increases the manoeuvrability of the butterflies, allowing them to escape from predators (Srygley \& Dudley, 1993). The slender abdominal profile of unpalatable butterflies may additionally be used as a signal of distastefulness (Chai \& Srygley, 1990), and therefore some Batesian mimics may attempt to simulate this (Srygley, 1994).

\section{Aim}

The aim of this experiment is to determine whether or not the hippocoonides morphs or the males are likely to be palatable or unpalatable on the basis of their morphology.

\section{Method \& Materials}

Ten male and nine hippocoonides female Papilio dardanus were used, and these were compared with ten Danaus chrysippus, following the general method of Chai \& Srygley (1990) followed. Measurements of the full body length of each specimen were made using a pair of Manostat measuring callipers (accurate to $0.05 \mathrm{~mm}$ ) by holding the abdomen of the live butterflies out straight, in line with the thorax, and measuring the length from the front of the head (not measuring the antennae) to the end of the genitalia. The width of the thorax was then measured by holding the butterflies' wings together dorsally, and tightening the callipers until they gripped the sides of the thorax at the point where the forewings joined the body. The ratio of body length to thorax width was then calculated for each specimen.

To ascertain the centre of mass for males and six of the hippocoonides females (three did not emerge properly from their pupal cases, and their abdomens were full of fluid), the bodies of freshly killed specimens were first weighed on an Oertling NA264 balance (accurate to $0.1 \mathrm{mg}$ ). Each body was then divided into several parts. First the head length was measured. This was done most accurately by measuring the diameter of the eye, which comprises most of the head. The head was then removed and weighed. The length of the thorax was measured next. The wings were then removed, the abdomen snipped off, and the thorax weighed. Finally, the abdomen was divided into three parts - the first, approximately three segments and containing the crop, the second approximately two segments containing the reproductive tracts, and the final part comprising three segments and containing the sclerotised genetalia. Each length of abdomen was measured, and then weighed.

To calculate the centre of mass of the insect, each section which had been weighed and measured was assumed to be of uniform density, and that the centre of mass lay on the axis of symmetry of the body. This assumption meant that the centre of mass of each section lay halfway down its length, and hence the turning force of each section around the head end could be calculated. 
The distance of the centre of mass of the entire insect from the head was thus the sum of these turning moments divided by the total mass of the insect (see Figure 6-2).

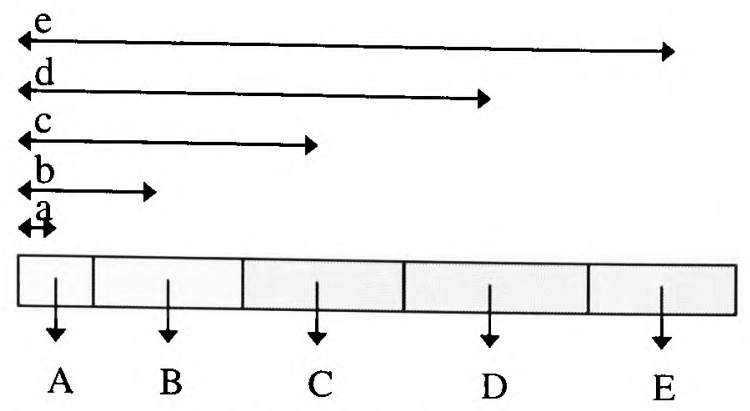

Figure 6-2 Representation of the calculation of the centre of mass of a butterfly

Centre of mass of entire insect is situated a distance $m$ from the head where:

$m=\frac{a A+b B+c C+d D+e E}{A+B+C+D+E}$

If the wing base of the insect is assumed to be in the centre of the thorax (distance $b$ from the head), then the centre of mass of the insect is situated a distance $(m-b)$ from the wing base. This distance can then be expressed as a fraction of the total length of the body.

\section{Results}

Thorax width: body length ratio.

\begin{tabular}{|c|c|c|c|}
\hline $\begin{array}{c}\text { Male } \\
\text { Specimen }\end{array}$ & $\begin{array}{c}\text { Thorax width } \\
(\mathbf{m m})\end{array}$ & $\begin{array}{c}\text { Body length } \\
\text { (mm) }\end{array}$ & Ratio \\
\hline 1 & 4.05 & 27.05 & 6.679 \\
\hline 2 & 3.60 & 24.25 & 6.736 \\
\hline 3 & 4.00 & 24.55 & 6.063 \\
\hline 4 & 4.10 & 28.80 & 7.024 \\
\hline 5 & 3.85 & 25.55 & 6.636 \\
\hline 6 & 3.35 & 24.00 & 7.164 \\
\hline 7 & 4.65 & 28.20 & 6.065 \\
\hline 8 & 4.75 & 27.55 & 5.800 \\
\hline 9 & 5.10 & 26.00 & 5.098 \\
\hline 10 & 4.45 & 27.95 & 6.281 \\
\hline MEAN & $\mathbf{4 . 1 9}$ & $\mathbf{2 6 . 3 6}$ & $\mathbf{6 . 3 5 5}$ \\
\hline
\end{tabular}

\begin{tabular}{|c|c|c|c|}
\hline $\begin{array}{c}\text { Female } \\
\text { Specimen }\end{array}$ & $\begin{array}{c}\text { Thorax width } \\
(\mathbf{m m})\end{array}$ & $\begin{array}{c}\text { Body length } \\
(\mathbf{m m})\end{array}$ & Ratio \\
\hline 1 & 5.10 & 29.05 & 5.696 \\
\hline 2 & 4.80 & 27.05 & 5.635 \\
\hline 3 & 4.85 & 27.10 & 5.588 \\
\hline 4 & 3.70 & 26.10 & 7.054 \\
\hline 5 & 4.35 & 31.45 & 7.230 \\
\hline 6 & 4.65 & 39.80 & 8.559 \\
\hline 7 & 4.85 & 26.10 & 5.381 \\
\hline 8 & 4.20 & 25.15 & 5.988 \\
\hline 9 & 4.40 & 24.20 & 5.500 \\
\hline MEAN & $\mathbf{4 . 5 4}$ & $\mathbf{2 8 . 4 4}$ & $\mathbf{6 . 2 9 2}$ \\
\hline
\end{tabular}

Table 6-5 The ratio of body length to thorax width in ten males and nine hippocoonides females of Papilio dardanus 


\begin{tabular}{|c|c|c|c|c|}
\hline Specimen & Sex & $\begin{array}{c}\text { Thorax width } \\
(\mathbf{m m})\end{array}$ & $\begin{array}{c}\text { Body length } \\
(\mathbf{m m})\end{array}$ & Ratio \\
\hline 1 & $\mathrm{~F}$ & 4.15 & 23.40 & 5.639 \\
\hline 2 & $\mathrm{~F}$ & 3.95 & 22.35 & 5.658 \\
\hline 3 & $\mathrm{M}$ & 4.1 & 23.40 & 5.707 \\
\hline 4 & $\mathrm{~F}$ & 3.85 & 23.60 & 6.130 \\
\hline 5 & $\mathrm{M}$ & 4.2 & 24.20 & 5.762 \\
\hline 6 & $\mathrm{M}$ & 3.8 & 25.05 & 6.592 \\
\hline 7 & $\mathrm{~F}$ & 3.7 & 22.1 & 5.973 \\
\hline 8 & $\mathrm{M}$ & 3.95 & 25.15 & 6.367 \\
\hline 9 & $\mathrm{M}$ & 4.15 & 23.55 & 5.675 \\
\hline 10 & $\mathrm{M}$ & 3.7 & 22.2 & 6.000 \\
\hline \multicolumn{2}{|c|}{ MEAN } & $\mathbf{3 . 9 6}$ & $\mathbf{2 3 . 5 0}$ & $\mathbf{5 . 9 2 3}$ \\
\hline
\end{tabular}

Table 6-6 The ratio of body length to thorax width in ten Danaus chrysippus

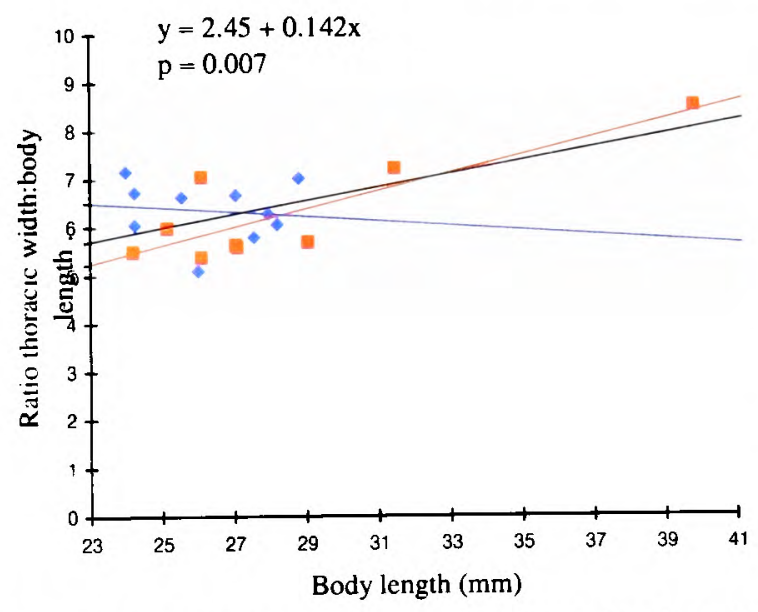

Figure 6-3 Graph of the ratio thoracic width:body length against body length in male (blue diamonds) and female (red squares) Papilio dardanus. Linear regression lines shown for males and females (in colour) and for the data as a whole (black)

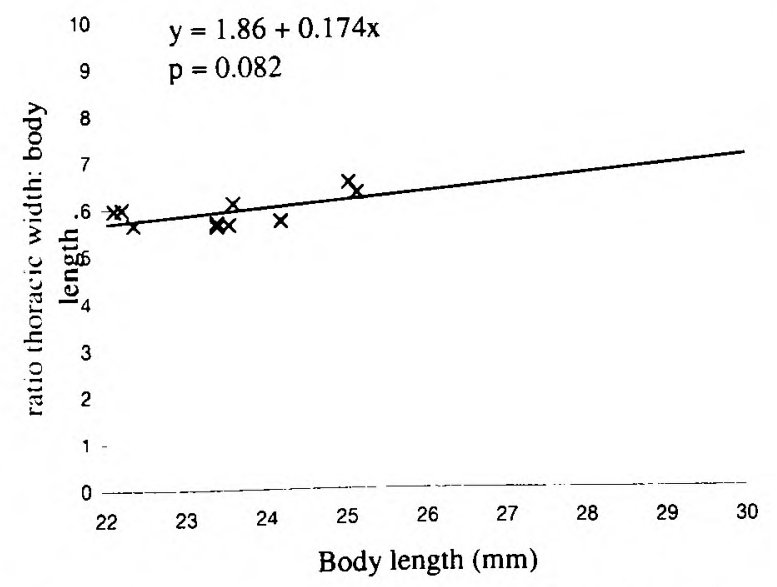

Figure 6-4 The ratio of body length to thoracic width against body length for Danaus chrysippus
A graph of the ratio of thoracic width to body length for males and females of Papilio dardanus shows that the ratio does increase slightly with body length ( $\mathrm{p}=0.007$, see Figure $6-3$ ), but there is no significant difference between the sexes $(p=0.487$, Mann-Whitney test, two-tailed $)$.

For Danaus chrysippus, there is a similar increase in ratio with body length, although this is not significant ( $\mathrm{p}=0.082$ ), and the body length shows much less variation. There is no significant difference between the sexes $(p=0.337$, MannWhitney test, two-tailed).

Chai \& Srygley (1990) plot body shape (the ratio of body length to thoracic width) against palatability (as measured by percentage eaten in feeding experiments) for 53 species of butterfly. This graph shows that highly palatable butterflies tend to have a ratio of between 4 and 6 , and unpalatable butterflies tend to have ratios of 8.5 and above (with a couple of exceptions). At intermediate values, where both Papilio dardanus and Danaus chrysippus appear to lie, the butterflies tend to have palatabilities of below $50 \%$. Figure 6-3 however, indicates that body length must be taken into account, as, at least in some species, butterflies 
3 however, indicates that body length must be taken into account, as, at least in some species, butterflies with longer bodies tend to have a higher ratio. Therefore comparing two species with differing overall body length may give misleading results. In this case, Danaus chrysippus is a smaller butterfly, and thus the ratio of body length to thoracic width may be lower than expected for an unpalatable species.

\begin{tabular}{|c|c|c|c|c|c|c|c|}
\hline & \multicolumn{9}{|c|}{ Turning force (mmm) around head of centre of mass of: } & \multicolumn{2}{c|}{ distance centre mass from: } \\
Male & Head & Thorax & Abdomen 1 & Abdomen 2 & Abdomen 3 & Head & Wing base \\
\hline 1 & 18.59 & 393.36 & 141.44 & 403.66 & 423.15 & 0.411 & 0.167 \\
\hline 2 & 14.5 & 259.55 & 86.45 & 305.61 & 277.38 & 0.417 & 0.168 \\
\hline 3 & 23.40 & 484.22 & 202.06 & 614.56 & 473.67 & 0.429 & 0.173 \\
\hline 4 & 16.06 & 544.36 & 243.89 & 962.32 & 279.38 & 0.379 & 0.164 \\
\hline 5 & 13.51 & 363.20 & 141.74 & 478.98 & 373.36 & 0.394 & 0.172 \\
\hline 6 & 8.19 & 274.80 & 68.15 & 280.79 & 274.3 & 0.411 & 0.172 \\
\hline 7 & 20.13 & 590.29 & 186.73 & 928.25 & 393.53 & 0.409 & 0.174 \\
\hline 8 & 23.03 & 580.97 & 490.78 & 827.52 & 528.41 & 0.442 & 0.187 \\
\hline 9 & 14.15 & 405.38 & 231.42 & 398.36 & 1045.23 & 0.402 & 0.130 \\
\hline 10 & 21.17 & 697.50 & 293.15 & 605.01 & 864.97 & 0.333 & 0.083 \\
\hline MEAN & $\mathbf{1 7 . 2 7}$ & $\mathbf{4 5 9 . 3 6}$ & $\mathbf{2 0 8 . 5 8}$ & $\mathbf{5 8 0 . 5 1}$ & 493.34 & $\mathbf{0 . 4 0 3}$ & $\mathbf{0 . 1 5 9}$ \\
\hline \hline Female & & & & & & & \\
\hline 1 & 23.00 & 680.21 & 716.94 & 1343.65 & 1293.86 & 0.478 & 0.245 \\
\hline 2 & 15.93 & 578.10 & 585.09 & 1113.81 & 971.19 & 0.525 & 0.264 \\
\hline 3 & 16.25 & 509.72 & 308.36 & 1058.64 & 818.35 & 0.521 & 0.260 \\
\hline 4 & 10.35 & 401.79 & 417.15 & 645.44 & 713.45 & 0.458 & 0.232 \\
\hline 5 & 15.89 & 857.21 & 1156.32 & 2155.43 & 2169.14 & 0.486 & 0.281 \\
\hline 6 & 9.90 & 539.28 & 810.65 & 2049.27 & 1127.30 & 0.386 & 0.228 \\
\hline MEAN & $\mathbf{1 5 . 2 2}$ & $\mathbf{5 9 4 . 3 8}$ & $\mathbf{6 6 5 . 7 5}$ & $\mathbf{1 3 9 4 . 3 7}$ & $\mathbf{1 1 8 2 . 2 1}$ & $\mathbf{0 . 4 7 6}$ & $\mathbf{0 . 2 5 2}$ \\
\hline
\end{tabular}

Table 6-7 Calculation of the centre of mass of male and female Papilio dardanus. The distances of the centre of mass from the head and wing base are given as proportions of the total length of the body.

\begin{tabular}{|c|c|c|c|c|c|c|c|c|}
\hline \multicolumn{6}{|c|}{ Specimen } & \multicolumn{6}{c|}{ Turning force (mgmm) around head of centre of mass of: } & \multicolumn{2}{c|}{ distance centre mass from: } \\
& Sex & Head & Thorax & Abdomen 1 & Abdomen 2 & Abdomen 3 & Head & Wing base \\
\hline 1 & F & 9.0 & 461.76 & 418.5 & 934.95 & 174.3 & 0.432 & 0.210 \\
\hline 2 & F & 6.6 & 289.44 & 226.4 & 493.65 & 154.8 & 0.403 & 0.389 \\
\hline 3 & M & 8.4 & 445.72 & 203.4 & 487.5 & 359.9 & 0.389 & 0.174 \\
\hline 4 & F & 7.91 & 357.64 & 233.2 & 680.52 & 207.6 & 0.387 & 0.189 \\
\hline 5 & M & 8.50 & 495.33 & 255.0 & 433.92 & 402.6 & 0.406 & 0.170 \\
\hline 6 & M & 7.65 & 516.88 & 308.73 & 716 & 322.64 & 0.414 & 0.190 \\
\hline 7 & F & 7.28 & 293.76 & 172.37 & 422.30 & 273.75 & 0.408 & 0.190 \\
\hline 8 & M & 9.18 & 608.06 & 363.05 & 719.82 & 475.58 & 0.437 & 0.203 \\
\hline 9 & M & 11.99 & 563.55 & 326.12 & 689.27 & 341.94 & 0.414 & 0.179 \\
\hline \multicolumn{2}{l}{ MEAN } & $\mathbf{8 . 5 0}$ & $\mathbf{4 4 8 . 0 2}$ & $\mathbf{2 7 8 . 5 5}$ & $\mathbf{6 1 9 . 7 7}$ & $\mathbf{3 0 1 . 4 6}$ & $\mathbf{0 . 4 1 0}$ & $\mathbf{0 . 1 8 8}$ \\
\hline
\end{tabular}

Table 6-8 The position of the centre of mass (in body length units) of Danaus chrysippus 


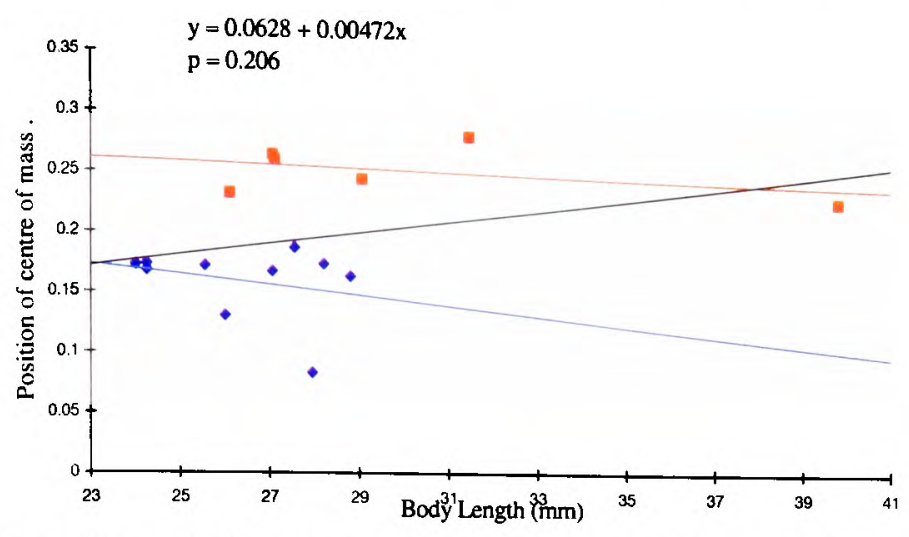

Figure 6-5 Graph of the distance of the centre of mass from the wing base of male (blue diamonds) and female (red squares) Papilio dardanus (in body length units) against body length. Linear regression lines shown in colour for each sex and in black for both taken together.

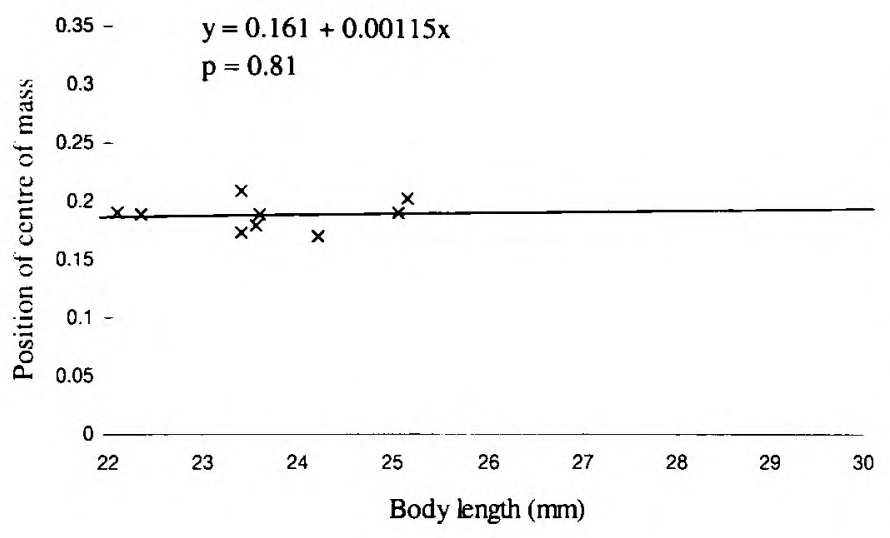

Figure 6-6 Graph of the distance of the centre of mass (in body length units) from the wing base of Danaus chrysippus.
A graph of the data for Papilio dardanus (see Figure 6-5) shows that the position of the centre of mass for the species does not vary significantly with the length of the body ( $p=0.206)$, and shows that females have a centre of mass set consistently further posterior than males ( $p=0.0014$; Mann-Whitney test, two-tailed).

A graph of the data for Danaus chrysippus (see Figure 6-6) indicates that again the centre of mass does not vary significantly with body length $(p=0.81$ ), and also that the position of the centre of mass in females is not significantly different from that in males ( $\mathrm{p}=0.27$; Mann-Whitney test, two-tailed). The females of Papilio dardanus, however, have a centre of mass positioned significantly further back than Danaus chrysippus $(\mathrm{p}=0.0018$; MannWhitney test, two-tailed).
Srygley and Dudley (1992), and Srygley (1994) give data for the percentage palatability and the position of the centre of mass for 51 species of butterfly. A graph of this can be seen in Figure 6-7. The highly palatable butterflies tend to cluster with a centre of body mass between 0.15 and 0.19 bodylength units from the wing base. The mid- to unpalatable species tend to have centres of mass between 0.19 and 0.25 (the Batesian mimics are reported to be at the extreme end of this

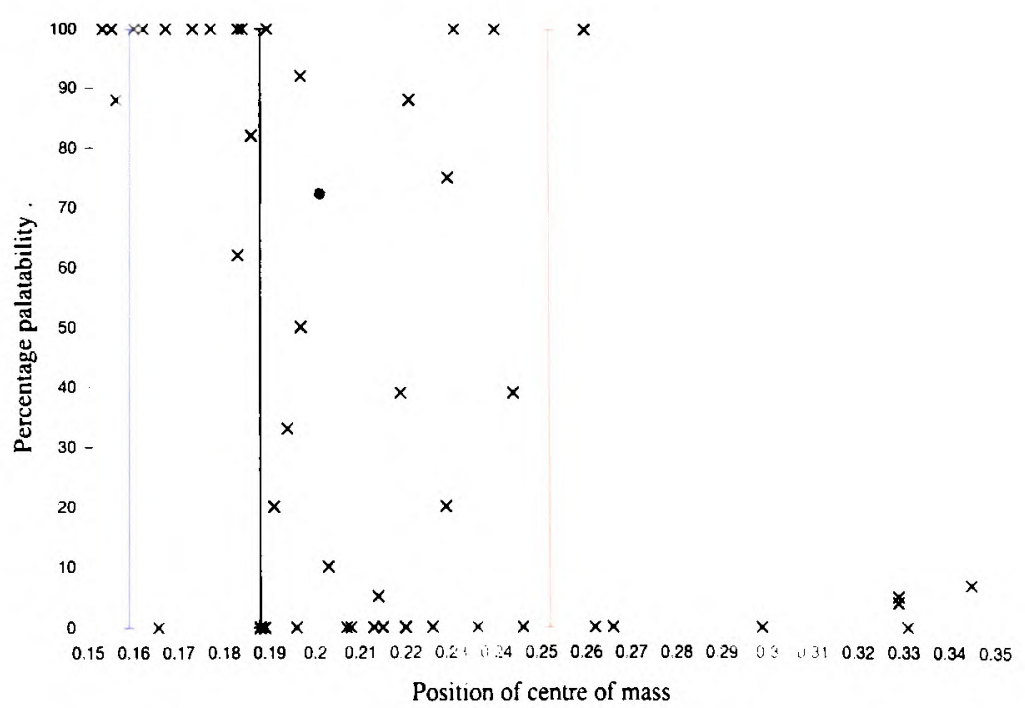

Figure 6.7 A graph of position of centre of mass against palatability in butterflies, from data by Srygley \& Dudley (1992) and Srygley (1994). Position of male Papilio dardanus shown as blue line, female as red, Danaus chrysippus as black 
distribution, with centres of mass between 0.2-0.25 body-units from the wing base). There is another cluster of unpalatable butterflies with a centre of body mass greater than 0.3 body lengths from the wing base.

The overall species mean position of the centre of mass $(0.194$ body lengths from the wing base) for Papilio dardanus therefore falls within the distribution of the mid- to unpalatable butterflies. The males are in the range for highly palatable butterflies and the females being in the normal range for palatable, Batesian mimics. The mean position of the centre of mass in Danaus chrysippus also falls in the range for mid- to unpalatable butterflies.

\section{Discussion}

The results of the both sets of data gathered for Papilio dardanus indicates that it is a Batesian mimic. From the evidence of the position of the centre of mass, it appears that the females of Papilio dardanus may not have shifted their centre of mass slightly in order to mimic their unpalatable models (Srygley, 1994), since their centre of mass is actually positioned further back than that of one of their models, Danaus chrysippus, hence putting them at greater risk than would be necessary to mimic the shape of their models. This effect is likely to be due simply to the heavy load of eggs carried by the females. Srygley (1994) did not analyse data for males and females separately, and so in order to compare the data for Papilio dardanus to that presented in that paper (Srygley, 1994) it is necessary to take a mean value for the species.

The fact that the centre of mass is set further posterior in female Papilio dardanus means that they will not be as aerobatically manoeuvrable as males (Srygley \& Dudley, 1993). This disadvantage could be a contributory factor causing female butterflies to be mimetic in species where males are not. Not only is there an evolutionary pressure for them to survive longer and lay more eggs (whilst males only need to mate), but the egg load they carry, by moving the centre of mass away from the wing base, causes them to be more prone to attack from birds (Srygley \& Dudley, 1993). This is in accordance with data on wing damage collected by Ohsaki (1995), who found that non-mimetic females of Papilio polytes appeared to suffer more bird attacks than males (although mimetic females appeared to receive as few attacks as males and their models, Pachiliopta aristolochiae). The fact that females of Danaus chrysippus did not have a significantly different positioning of the centre of mass is interesting. It is possible that different egg loads carried by females of different species can create different evolutionary pressures on the sexes in some species, but not in others. 


\section{$\underline{\text { Conclusions }}$}

Papilio dardanus appears to be completely palatable to starlings. Danaus chrysippus, however, also appeared to be palatable to the starlings, although it is possible that the individual butterflies used were from a population which did not sequester cardiac glycosides efficiently. The measurements of the morphology of the two species indicated that they both lie in a region where it is difficult to predict the palatability with any accuracy. However, the males of Papilio dardanus had a centre of mass which indicated that they were completely palatable, and therefore it seems likely that the species as a whole is palatable.

The morphology of the butterflies also demonstrated that the females of Papilio dardanus are likely to be less manoeuvrable than the males in flight, due to the position of their centre of mass. This is likely to make them much easier prey for birds to catch (Srygley \& Dudley, 1993), and hence increases the pressure on females to avoid attack. This could be a contributing factor to the phenomenon of female-limited mimicry in butterflies. Turner (1978) reviewed this topic of debate, and concluded that there was no evidence that female butterflies were subject to more predation than males, but the evidence from Ohsaki (1995), and the sex difference in the degree of flight manoeuvrability now suggests that they are. The theory that female choice maintains male polymorphism (Belt, 1874), which was favoured by Turner (1978) has very little evidence to support it (female choice in butterflies appears only to have been demonstrated in Danaus chrysippus, in which both sexes are in fact polymorphic; Smith, 1984). In Papilio dardanus, the female-limited mimicry is spectacular. Male sexual selection appears to be operating (Cook et al., 1994 and see also Chapter 5), whilst female choice has not yet been demonstrated (see Chapter 5). The females fly under thicker cover (Nigel Venters ${ }^{*}$, pers. comm.), and at different times of day from the males (Cook et al., 1994), and the measurements taken in Experiment 6-6 indicate that they are likely to be more easily caught by birds. The evidence, therefore, for Papilio dardanus, suggests that an increased predation risk for females may be a more important factor in the evolution of female-limited mimicry than the action of female choice on male coloration. The evidence of the position of the centre of mass in Danaus chrysippus indicates that in this species there is no difference between the aerobatic ability of the males and the females. Therefore in this species the evolutionary pressures on the two sexes are likely to be much more similar. Differences in the egg laying strategies of different species may therefore have a profound effect on the evolutionary pressures on the two sexes, and may be an explanation for the existence of sex-limited mimicry in some species and its absence in others.

Measurements by Srygley (1994) from mimetic and non-mimetic species showed that Batesian mimics on the whole tended to have a centre of mass positioned slightly further back than non-mimics. $\mathrm{He}$ interpreted this as possible evidence of 'locomotor mimicry'. However, it is also possible that the 
position of the centre of mass of those species had meant that they were more likely to evolve mimicry, due to the fact that they were slightly less agile in flight. Further measurements of both sexes in a range of sex-limited and sex-unlimited mimetic species and their models may help clarify this issue.

\section{Acknowledgements}

Many thanks to everyone who helped supply butterflies for this experiments, especially John Calvert at Stratford Butterfly Farm and Pierre Le Roux in South Africa. Many thanks also to Dave Wilson for providing and looking after the starlings, and to Julian Howe for the chicks.

I am also very grateful to Bob Srygley for suggesting the method for taking measurements of the butterflies, and for the loan of the calipers, and to Nicky Warren for the use of the balance.

\section{References}

Belt, T. 1874. The naturalist in Nicaragua. Murray, London.

Bernardi, G. 1963. Quelques aspects zoogéographiques du mimétisme chez les Lépidpidoptères. Proceedings, $16^{\text {th }}$ International Congress of Zoology, Washington 4, 161-166.

Brower, J.V.Z. 1960. Experimental studies of mimicry. IV The reactions of starlings to different proportions of models and mimics. Am. Nat. 94, 271-282.

Brower, L.P. 1969. Ecological chemistry. Scientific American, 22-29.

Brower, L.P. 1984. Chemical defence in butterflies. In The Biology of Butterflies (ed. Vane-Wright, R.I. \& Ackery, P.R.) Princetown University Press, New Jersey.109-134.

Brower, L.P., Brower, J.V.Z. \& Corvino, J.M. 1967. Plant poisons in a terrestrial food chain. Proc. Natn. Acad. Sci. U.S.A. 57, 893-898.

Brower, L.P., Edmunds, M. \& Moffitt, C.M. 1975. Cardenolide content and palatability of a population of Danaus chrysippus butterflies from West Africa. J. Ent. (A) 49, 183-196.

Brower, L.P., Gibson, D.O., Moffitt, C.M. \& Panchen, A.L. 1978. Cardenolide content of Danaus chrysippus butterflies from three areas of East Africa. Biol. J. Linn. Soc. 10, $251-273$.

Brower, L.P., Ryerson, W.N., Coppinger, L.L., \& Glazier, S.C. 1968. Ecological chemistry and the palatability spectrum. Science 161, 1349-51.

Carpenter, G.D.H. 1941. Observations and experiments in Africa by the late C.F.M. Swynnerton on wild birds eating butterflies and the preference shown. Proc. Linn. Soc. Lond. 154, 10-46.

Chai, P. \& Srygley, R.B. 1990. Predation and the flight, morphology and temperature of Neotropical rain-forest butterflies. Am. Nat. 135, 748-765.

Charlesworth, D. \& Charlesworth, B. 1976. Theoretical genetics of Batesian mimicry III Evolution of dominance. J. Theor. Biol. 55, 325-337. 
Clarke, C.A., Clarke, F.M.M., Collins, S.C., Gill, A.C.L., Turner, J.R.G. 1985. Male-like females, mimicry, and transvestism in swallowtail butterflies. Systematic Entomology 10, 257-283.

Clarke, C.A. \& Sheppard, P.M. 1963. Interactions between major genes and polygenes in the determination of the mimetic patterns of Papilio dardanus. Evolution 17, 404-413.

Cook, S.E., Vernon, J.G., Bateson, M., Guilford, T. 1994. Mate choice in the polymorphic African swallowtail butterfly, Papilio dardanus: male-like females may avoid sexual harrassment. Animal Behaviour 47, 389-397.

Dixon, C.A., Erikson, J.M., Kellett, D.N. \& Rothschild, M. 1978. Some adaptations between Danaus plexippus and its food plant, with notes on Danaus chrysippus and Euploea core. J. Zool., Lond. 185, 437-467.

Fink, L.S. \& Brower, L.P. 1981. Birds can overcome the cardenolide defences of Monarch butterflies in Mexico. Nature 291, 67.

Ford, E.B. 1936. The genetics of Papilio dardanus Brown (Lep.). Transactions of the Royal Entomological Society of London 85, 435-466.

Ford, E.B. 1955. Moths. London.

Gittleman, J.L. \& Harvey, P.H. 1980. Why are distasteful prey not cryptic? Nature 286, 149-150.

Glendinning, J.I. \& Brower, L.P. 1990. Feeding and breeding responses of five mice species to overwintering aggregations of the Monarch butterfly. Journal of Animal Ecology 59, 1091-1112.

Meinwald, J., Boriack, C.J., Schneider, D., Boppré, M., Wood, W.F. \& Eisner, T. 1974. Volatile ketones in the hairpencil secretion of danaid butterflies (Amauris \& Danaus), Experientia 30, 721722.

O'Donald, P. \& Barrett, J.A. 1973. Evolution of dominance in polymorphic Batesian mimicry. Theoretical Population Biology 4, 173-192.

Ohsaki, N. 1995. Preferential predation of female butterflies. Nature 378, 173-5.

Poulton, E.B. 1924. Papilio dardanus. The most interesting butterfly in the world. Journal of the East African and Ugandan Natural History Society 20, 4-22.

Rothschild, M., Moore, B.P. \& Brown, W.V. 1984. Pyrazines as warning odour components in the monarch butterfly, Danaus plexippus, and in moths of the genera Zygaena and Amata. Biol. J. Linn. Soc. 23, 375-380.

Rowe, C. \& Guilford, T. 1996. Hidden colour aversions in domestic chicks triggered by pyrazine odours of insect warning displays. Nature 383, 520-522.

Sheppard, P.M. 1967. Natural selection and heredity ( $3^{\text {rd }}$ edn.). Hutchinson, London.

Speed, M.P. 1993. Müllerian mimicry and the psychology of predation. Anim. Behav. 45, 571-580.

Srygley, R.B. 1994. Locomotor mimicry in butterflies? The associations of positions of centres of mass among groups of mimetic, profitable prey. Phil. Trans. R. Soc. Lond. B 343, 145-155.

Srygley, R.B. \& Dudley, R. 1993. Correlations of the position of center of body mass with butterfly escape tactics. J. Exp. Biol. 174, 155-166. 
Srygley, R.B. \& Kingsolver, J.G. 1998. Red-wing blackbird reproductive behaviour and the palatability, flight performance, and morphology of temperate pierid butterflies (Colias, Pieris, and Pontia). Biol. J. Linn. Soc. 64 (1), 41-55.

Swynnerton, C.F.M. 1915. Birds in relation to their prey. Experiments on wood-hoopoes, small hombillsa, and a babbler. Jl. S. Afr. Orn. Un. 1915, 22-108.

Swynnerton, C.F.M. 1919. Experiments and observations bearing on the explanation of form and colouring, 1908-1913, Africa. Zool. J. Linn. Soc. 33, 203-385.

Trimen, R. 1868. On some remarkable mimetic analogies among African butterflies. Transactions of the Linnean Society of London 26, 497-522.

Turner, J.R.G. 1963. Geographical variation and evolution in the males of the butterfly Papilio dardanus Brown (Lepidoptera: Papilionidae). Trans. Roy. Ent. Soc. Lond. 115, 239-259.

Turner, J.R.G. 1978. Why male butterflies are non-mimetic: natural selection, sexual selection, group selection, modification and sieving. Biol. J. Linn. Soc. 10, 385-432.

Van Bemmelen, J.F. 1922. The wing-design of mimetic butterflies. Proceedings, Section of Sciences, Koninklijke Nederlandse Akademie Wetensschappen 23, 877-886.

Vane-Wright, R.I., Raheem, D.C., Cieslak, A., Vogler, A.P. In press. Evolution of the mimetic African swallowtail butterfly Papilio dardanus: molecular data confirms relationships with $P$. phorcas and P. constantinus. Biol. J. Linn. Soc.

Vane-Wright, R.I. \& Smith, C.R. 1991. Phylogenetic relationships of three African swallowtail butterflies, Papilio dardanus, $P$. phorcas, and P. constantinus: a cladistic analysis (Lepidoptera: Papilionidae). Systematic Entomology 16: 275-291. 


\section{Chapter Seven}

\section{Mathematical model and general conclusions}

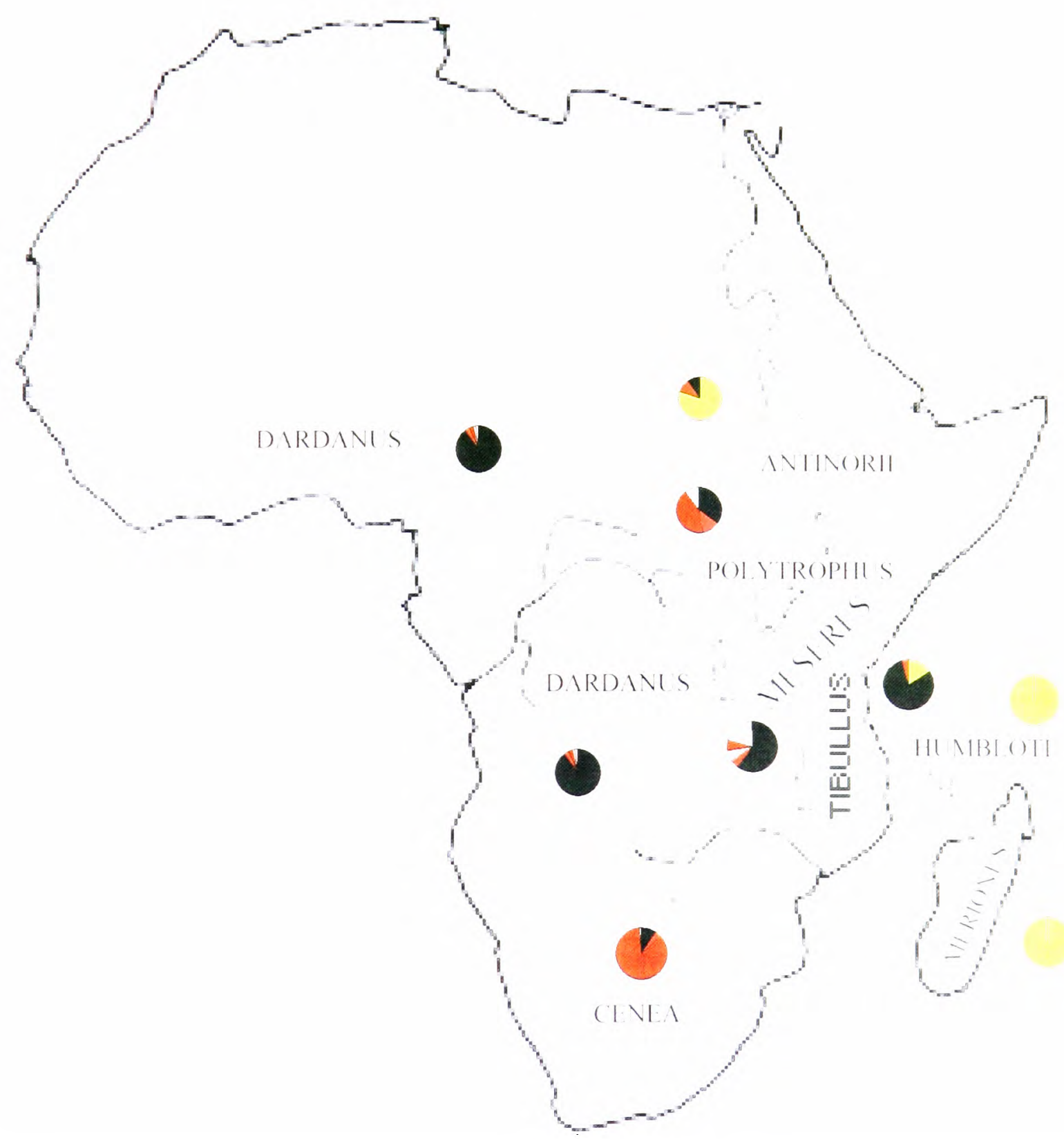

Figure 7-1 A map showing the distributions of the different geographical races of Papilio dardanus, and the approximate proportions of different morphs recorded from each race (yellow = andromorphic, black = hippocoonides, brown = cenea, red = trophonius, orange $=$ mainly orange morphs, white $=$ other, non-mimetic forms). Data from Appendix 1.

"The genetics of Papilio dardanus should in the future prove an exceedingly fruitful study..."

E.B. Ford (1936) 


\section{Introduction}

Each population of Papilio dardanus has a different balance of morphs. If it is assumed that the proportions of the morphs in the various races of Papilio dardanus are at equilibrium, then the various factors which affect the frequencies of the morphs must balance each other.

The factors which might need to be taken into consideration are:

1) Survival without predation (e.g. heat tolerance) - assumed to be constant for each morph.

2) Predation - for mimetic morphs, dependent on the relative frequencies of models and mimics.

3) Mate Choice - two components:

a) Male mate choice - possibly frequency dependent, from the results of Chapter 5 .

b) Female mate choice - assumed to be constant.

4) Fecundity - assumed to be constant for each morph.

5) Survival of offspring - assumed to be constant for each morph.

For the polymorphism to be balanced, the sum of all these factors must be equal for all morphs.

Chapters 5 and 6 of this thesis have been concerned with two of these factors: mate choice and predation, and therefore the results of the experiments in these chapters can be used to construct a speculative mathematical model of the populations of Papilio dardanus. Comparing the results of such a model with the population balances observed in the different races of Papilio dardanus may help demonstrate whether the factors investigated are likely to have been correctly assessed. Such a model should demonstrate the general effects on the population balance of factors such as learning in mate choice. It can also be used to explore different ways in which the populations may have evolved, through introducing new morphs to a theoretical ancestral population.

\section{$\underline{\text { Aims }}$}

The aim of this chapter is to construct a mathematical model of a polymorphic population of Papilio dardanus using all the available information about the factors affecting the survival and reproduction of the different morphs. By doing this, the strength of factors which have not been evaluated can be assessed, and conclusions drawn about the evolution and maintenance of the different morphs in various populations. 


\section{Method}

Each of the factors listed in the Introduction to this chapter is evaluated below, and where possible, values obtained for the variables to allow them to be incorporated into a mathematical model of the population. Those parameters for which values are not available are then varied until the population balance seen in the different races is achieved. This gives an estimate of the unknown parameters, and the model can then be used to investigate the effect of changing conditions and the evolution of new morphs.

\section{Factor 1 Survival without predation, fecundity, and survival of offspring}

\section{Introduction}

Sir Cyril Clarke hand-paired many generations of Papilio dardanus whilst researching the genetic basis of the morph patterns. He kept detailed brood books in the form of diaries, with daily information about every individual, allowing the number of eggs laid by each female, the length each individual spends at each stage of the lifecycle, and the mortality to be extracted. This huge amount of data can be utilised to search for significant differences in fecundity between morphs, and his data also may suggest whether or not there is a difference in the survival rates of the adults and larvae without predation (although in an artificial situation).

\section{Method}

The information from a number of Sir Cyril Clarke's brood books (between March 1981 and October 1997) was entered into a database (Microsoft Access 95), and was then queried to look for significant differences between the fecundity and survival at different stages of the lifecycle of different morphs. With Sir Cyril Clarke's permission, the database is available over the Web at:

http:Ilwww.linacre.ox.ac.uklresearchldardanus_genetics

(This site requires Internet Explorer, Version 4 or above)

The mean values of longevity of the adults, the number of days spent at each stage of the lifecycle, the number of eggs laid, the number of resulting larvae, and the number of resulting pupae were calculated for each morph (across all the races studied). This was done using the 'Analyse by Morph' pages on the website. 


\section{Results}

The mean values for each parameter for each morph are shown in Table 7-1.

\begin{tabular}{|c|c|c|c|c|c|}
\hline Morph & hippocoonides & cenea/protocenea & natalica & lamborni & trimeni \\
\hline $\begin{array}{c}\text { no. days as } \\
\text { adult }\end{array}$ & $\begin{array}{c}8.56 \\
(\sigma=3.92, \mathrm{n}=71)\end{array}$ & $\begin{array}{c}9.07 \\
(\sigma=4.25, \mathrm{n}=45)\end{array}$ & $\begin{array}{c}7.14 \\
(\sigma=2.55, \mathrm{n}=7)\end{array}$ & $\begin{array}{c}5.38 \\
(\sigma=1.92, \mathrm{n}=8)\end{array}$ & $\begin{array}{c}3.50 \\
(\sigma=3.10, \mathrm{n}=4)\end{array}$ \\
\hline $\begin{array}{c}\text { no. days as } \\
\text { egg }\end{array}$ & 5.40 & 5.15 & 5.46 & 5.40 \\
$(\sigma=1.24, \mathrm{n}=76)$ & $(\sigma=0.73, \mathrm{n}=72)$ & $(\sigma=1.61, \mathrm{n}=13)$ & $(\sigma=1.65, \mathrm{n}=10)$ & $\begin{array}{c}5.33 \\
(\sigma=2.07, \mathrm{n}=6)\end{array}$ \\
\hline $\begin{array}{c}\text { no. days as } \\
\text { larva }\end{array}$ & 30.23 & 33.56 & 30.00 & 32.6 & 32.33 \\
$(\sigma=4.69, \mathrm{n}=62)$ & $(\sigma=3.81, \mathrm{n}=63)$ & $(\sigma=5.16, \mathrm{n}=13)$ & $(\sigma=6.80, \mathrm{n}=10)$ & $(\sigma=4.93, \mathrm{n}=6)$ \\
\hline $\begin{array}{c}\text { no. days as } \\
\text { pupa }\end{array}$ & 13.77 & 14.02 & 13.15 & 13.5 & 13.17 \\
\hline $\begin{array}{c}\text { no. eggs } \\
\text { laid }\end{array}$ & $(\sigma=2.12, \mathrm{n}=88)$ & $(\sigma=2.36, \mathrm{n}=92)$ & $(\sigma=2.34, \mathrm{n}=13)$ & $(\sigma=1.78, \mathrm{n}=10)$ & $(\sigma=3.60, \mathrm{n}=6)$ \\
\hline $\begin{array}{c}\text { no. larvae } \\
\text { emerged }\end{array}$ & $(\sigma=10.39, \mathrm{n}=132)$ & $(\sigma=12.52, \mathrm{n}=111)$ & $(\sigma=20.90, \mathrm{n}=7)$ & $(\sigma=6.89, \mathrm{n}=17)$ & $(\sigma=11.44, \mathrm{n}=7)$ \\
\hline $\begin{array}{c}\text { no. larvae } \\
\text { pupated }\end{array}$ & 2.06 & 3.60 & 11.71 & 2.65 & 5.71 \\
$(\sigma=5.97, \mathrm{n}=132)$ & $(\sigma=9.33, \mathrm{n}=111)$ & $(\sigma=17.71, \mathrm{n}=7)$ & $(\sigma=6.27, \mathrm{n}=17)$ & $(\sigma=7.14, \mathrm{n}=7)$ \\
\hline
\end{tabular}

Table 7-1 The mean values of the parameters for each morph, with standard deviations, as taken from Sir Cyril Clarke's data

It can be seen that the fecundity measurements (the last three rows) have very large standard deviations. This is because the distribution is very odd, with many females not laying at all, but those that do having many offspring. This distribution makes the data very difficult to analyse (and removing the females who did not lay would influence the result considerably). Therefore it was not possible to look for differences in fecundity. The means of the other categories, however, were compared using a Welch-Satterthwaite solution (which does not assume equal variances in the two groups), shown in Table 7-2, Table 7-3, and Table 7-4.

\begin{tabular}{|c|c|c|c|c|}
\hline & $\begin{array}{c}\text { hippocoonides } \\
\text { vs. cenea }\end{array}$ & $\begin{array}{c}\text { hippocoonides } \\
\text { vs. natalica }\end{array}$ & $\begin{array}{c}\text { hippocoonides } \\
\text { vs. lamborni }\end{array}$ & $\begin{array}{c}\text { hippocoonides } \\
\text { vs. trimeni }\end{array}$ \\
\hline no. days as adult & $\mathrm{p}=0.197$ & $\mathrm{p}=0.059$ & $\mathrm{p}=0.0001$ & $\mathrm{p}<0.00001$ \\
\hline no. days as egg & $\mathrm{p}=0.139$ & $\mathrm{p}=0.861$ & $\cdot \mathrm{p}=0.990$ & $\mathrm{p}=0.923$ \\
\hline no. days as larva & $\mathrm{p}<0.00001$ & $\mathrm{p}=0.747$ & $\mathrm{p}=0.020$ & $\mathrm{p}=0.067$ \\
\hline no. days as pupa & $\mathrm{p}=0.266$ & $\mathrm{p}=0.191$ & $\mathrm{p}=0.555$ & $\mathrm{p}=0.477$ \\
\hline
\end{tabular}

Table 7-2 The p-values for comparisons between the values for various parameters for hippocoonides and the other morphs

\begin{tabular}{|c|c|c|c|}
\hline & $\begin{array}{c}\text { cenea vs. } \\
\text { natalica }\end{array}$ & $\begin{array}{c}\text { cenea vs. } \\
\text { lamborni }\end{array}$ & cenea vs. trimeni \\
\hline no. days as adult & $\mathrm{p}=0.019$ & $\mathrm{p}<0.00001$ & $\mathrm{p}=0.001$ \\
\hline no. days as egg & $\mathrm{p}=0.413$ & $\mathrm{p}=0.567$ & $\mathrm{p}=0.774$ \\
\hline no. days as larva & $\mathrm{p}=0.0001$ & $\mathrm{p}=0.291$ & $\mathrm{p}=0.241$ \\
\hline no. days as pupa & $\mathrm{p}=0.074$ & $\mathrm{p}=0.270$ & $\mathrm{p}=0.329$ \\
\hline
\end{tabular}

Table 7-3 The p-values for comparisons between the values for various parameters for cenea and the other morphs 


\begin{tabular}{|c|c|c|c|}
\hline & $\begin{array}{c}\text { natalica vs. } \\
\text { lamborni }\end{array}$ & $\begin{array}{c}\text { natalica vs. } \\
\text { trimeni }\end{array}$ & $\begin{array}{c}\text { lamborni vs. } \\
\text { trimeni }\end{array}$ \\
\hline no. days as adult & $\mathrm{p}=0.042$ & $\mathrm{p}=0.014$ & $\mathrm{p}=0.122$ \\
\hline no. days as egg & $\mathrm{p}=0.910$ & $\mathrm{p}=0.856$ & $\mathrm{p}=0.927$ \\
\hline no. days as larva & $\mathrm{p}=0.022$ & $\mathrm{p}=0.061$ & $\mathrm{p}=0.831$ \\
\hline no. days as pupa & $\mathrm{p}=0.569$ & $\mathrm{p}=0.989$ & $\mathrm{p}=0.715$ \\
\hline
\end{tabular}

Table 7-4 The p-values for comparisons between the values for various parameters for natalica and the other morphs, and lamborni with trimeni

So many pair-wise comparisons cause a statistical problem, as the probability that one of them is unrepresentative by chance increases with the number of p-values calculated. Therefore, the threshold of significance should be taken to be much lower than 0.05 . Using a simple Bonferroni adjustment, for this test, the significance level was increased to 0.00125 . This means that out of the pvalues calculated from means without very large standard deviations, only six are significant. Two of these are the comparisons between the lengths of time spent as a larva by cenea females and natalica and hippocoonides females. The others are the comparisons of longevity of the adults between both trimeni and lamborni morphs and both cenea and hippocoonides females.

\section{Discussion}

There are very few significant differences in the lifecycles of the different morphs of Papilio dardanus as recorded in an artificial breeding situation by Sir Cyril Clarke. One difference, between the time spent as a larva by cenea females and both natalica and hippocoonides females will make no difference to the mathematical model built in this chapter. The fact that lamborni and trimeni females appear to live slightly shorter in an artificial, predator-free environment may be significant, but the actual values, shown in Table 7-1 show that the value for the longevity of lamborni was based only on 8 individuals, and that for trimeni on only 4 individuals and therefore the sample size is very small. The longevity difference is also quite small in terms of days, and hence this difference has been disregarded as far as the mathematical model is concerned.

It is unfortunate that the figures on fecundity had distributions which made it difficult to make meaningful comparisons. Dissection of females of different morphs would allow the numbers of eggs carried by each to be counted, which might be a better estimate of their fecundity. However, fresh specimens of the morphs were not available, and so for this model, the fecundity of the morphs has also been assumed to be equal. 


\section{Factor 2 Mate choice}

\section{Introduction}

The experiments of Chapter 5, together with data from Cook et al. (1994) on male mate choice behaviour in the wild, give an idea of how the males choose females. As there is no evidence that females can determine the genotype of males, females are assumed to be mating at random. From the results of Experiment 5-3 it seems unlikely that there is a genetic factor governing the males' choice which is in linkage disequalibrium with the pattern-causing genes they carry, therefore the genotype of the males can be ignored.

From Experiments 5-1 and 5-2 it appears that males choose at random on their first encounter with a female, but subsequently their choice is affected by previous experience. This hypothesis fits the general trend recorded by Cook et al (1994) of the males preferring the most common morph (see Appendix 5 for a mathematical analysis of the data). Using this hypothesis, together with the data of Chapter 5, the probabilities of each possible mating can be calculated.

\section{Calculations}

See Appendix 1 for a summary of the notation used for the genes for pattern formation.

1) The probability of mating with a female of a given genotype on the first mating is simply the chance of meeting that genotype (proportion of the genotype in the population). Because of the dominance hierarchy of the genes, the phenotype rather than the genotype must be considered.

$$
\begin{aligned}
& \text { e.g. } \left.\quad \mathrm{p} \text { (mating with } \mathrm{H}^{\mathrm{c}} \mathrm{H}^{\mathrm{c}} \text { female on first mating }\right)=\left(\text { proportion of } \mathrm{H}^{\mathrm{c}} \mathrm{H}^{\mathrm{c}}\right) \\
& \mathrm{p}(\text { mating with cenea female on first mating })=\left(\text { proportion of } \mathrm{H}^{\mathrm{c}} \mathrm{H}^{\mathrm{c}}+\mathrm{h} \mathrm{H}^{\mathrm{c}}\right)
\end{aligned}
$$

2) The probability of mating with a female of a given genotype on the second mating is the probability of meeting that genotype multiplied by the probability that the male mated with a female of the same morph the first time (calculated from the results above) multiplied by the probability that the male chooses the same morph again (taken to be 0.75, from the results of Experiment 5-2) plus the probability that he mated with a female of one of the other morphs first time multiplied by the probability that the male would choose a different morph plus the same for the final morph ALL multiplied by the proportion of the given genotype in the population. 
e.g. p(mating with $\mathrm{H}^{\mathrm{c}} \mathrm{H}^{\mathrm{c}}$ female on second mating)

$$
\begin{aligned}
& =[\mathrm{p}(\text { mated cenea on first mating }) \times \mathrm{p}(\text { choosing cenea twice }) \\
& +\mathrm{p}(\text { mated hippocoonides on first mating }) \times \mathrm{p}(\text { choosing different morph }) \\
& +\mathrm{p}(\text { mated trophonius on first mating }) \times \mathrm{p}(\text { choosing different morph })] \\
& \left.\mathrm{x} \text { (proportion of } \mathrm{H}^{\mathrm{c}} \mathrm{H}^{\mathrm{c}} \text { in population }\right)
\end{aligned}
$$

To combine these two, the probability of mating with a given genotype in total is:

$p($ mating given genotype $)=[p($ this is first mating $) \times p($ mating with genotype on first mating) $]$

$+[\mathrm{p}$ (this is second mating) $\mathrm{x}$ (mating with genotype on second mating) $]$

\section{Results}

The probabilities of a male mating with each genotype, calculated above, can then be used in a

\begin{tabular}{|c|c|c|c|c|c|c|c|}
\hline & & hh & $\mathrm{hH}^{\mathrm{c}}$ & $\begin{array}{l}\text { male } \\
\mathrm{H}^{\mathrm{c}} \mathrm{H}^{\mathrm{c}}\end{array}$ & $\mathrm{hH}^{\mathrm{T}}$ & $\mathrm{H}^{\mathrm{c}} \mathrm{H}^{\mathrm{T}}$ & $\mathrm{H}^{\mathrm{T}} \mathrm{H}^{\mathrm{T}}$ \\
\hline \multirow{6}{*}{ female } & \multirow{6}{*}{$\begin{array}{c}\text { hh } \\
h H^{\mathrm{c}} \\
\mathrm{H}^{\mathrm{c}} \mathrm{H}^{\mathrm{c}} \\
\mathrm{h} \mathrm{H}^{\mathrm{T}} \\
\mathrm{H}^{\mathrm{c}} \mathrm{H}^{\mathrm{T}} \\
\mathrm{H}^{\mathrm{T}} \mathrm{H}^{\mathrm{T}}\end{array}$} & $\mathrm{M}_{\mathrm{hh}}$ & $\mathrm{M}_{\mathrm{hh}}$ & $\mathrm{M}_{\mathrm{hh}}$ & $\mathbf{M}_{\mathrm{hh}}$ & $\mathrm{M}_{\mathrm{hh}}$ & $\mathrm{M}_{\mathrm{hh}}$ \\
\hline & & $\mathbf{M}_{\mathrm{hc}}$ & $\mathrm{M}_{\mathrm{hc}}$ & $\mathrm{M}_{\mathrm{hc}}$ & $\mathrm{M}_{\mathrm{hc}}$ & $\mathrm{M}_{\mathrm{hc}}$ & $\mathrm{M}_{\mathrm{hc}}$ \\
\hline & & $\mathbf{M}_{\mathrm{cc}}$ & $\mathrm{M}_{\mathfrak{c c}}$ & $\mathrm{M}_{\mathrm{cc}}$ & $\mathrm{M}_{\mathrm{cc}}$ & $\mathrm{M}_{\mathrm{cc}}$ & $\mathrm{M}_{\mathrm{cc}}$ \\
\hline & & $\mathrm{M}_{\mathrm{hT}}$ & $\mathrm{M}_{\mathrm{hT}}$ & $\mathrm{M}_{\mathrm{hT}}$ & $\mathrm{M}_{\mathrm{hT}}$ & $\mathrm{M}_{\mathrm{hT}}$ & $\mathrm{M}_{\mathrm{hT}}$ \\
\hline & & $\mathbf{M}_{\mathrm{cT}}$ & $\mathrm{M}_{\mathrm{cT}}$ & $\mathbf{M}_{\mathrm{cT}}$ & $\mathrm{M}_{\mathrm{cT}}$ & $\mathrm{M}_{\mathrm{cT}}$ & $\mathrm{M}_{\mathrm{cT}}$ \\
\hline & & $\mathrm{M}_{\Pi \mathrm{T}}$ & $\mathrm{M}_{\mathrm{TT}}$ & $\mathbf{M}_{\mathrm{TT}}$ & $\mathbf{M}_{\mathrm{TT}}$ & $\mathbf{M}_{\mathrm{TT}}$ & $\mathbf{M}_{\Pi \mathrm{T}}$ \\
\hline
\end{tabular}
mating matrix to calculate the proportions of each genotype in the next generation. This consists of constructing a table of all the possible matings and the probabilities of each occurring (given the mate choice, calculated above):

Where $\mathbf{M}_{\mathrm{hh}}=\mathbf{p}($ male choosing hh female $)$

$\mathrm{M}_{\mathrm{hc}}=\mathrm{p}$ (male choosing $\mathrm{hH}^{\mathrm{c}}$ female)

$\mathbf{M}_{\mathrm{cc}}=\mathrm{p}\left(\right.$ male choosing $\mathrm{H}^{\mathrm{c}} \mathrm{H}^{\mathrm{c}}$ female)

$\mathrm{M}_{\mathrm{hT}}=\mathrm{p}$ (male choosing $\mathrm{hH}^{\mathrm{T}}$ female)

$M_{c T}=p\left(\right.$ male choosing $H^{\mathrm{c}} H^{\mathrm{T}}$ female)

$\mathbf{M}_{\mathrm{TT}}=\mathrm{p}\left(\right.$ male choosing $\mathrm{H}^{\mathrm{T}} \mathrm{H}^{\mathrm{T}}$ female)

Note the assumption that females do not choose males on the basis of their phenotype, and that males do not have a genetic morph preference which is linked to their own morph genotype, hence the genotype of the males makes no difference to the probability of them choosing a particular morph. 
From this matrix the proportions of each genotype in the next generation can be calculated by multiplying the probability of a given pairing occurring by the proportion of the offspring from that pairing which will be of each genotype and summing all the probabilities for each genotype.

For example, the proportion of hh in the next generation will be:

$\mathrm{M}_{h h}\left(\mathrm{P}_{h h}+0.5 \mathrm{P}_{\mathrm{hc}}+0.5 \mathrm{P}_{\mathrm{hT}}\right)+0.5 \mathrm{M}_{\mathrm{hc}}\left(\mathrm{P}_{\mathrm{hh}}+0.5 \mathrm{P}_{\mathrm{hc}}+0.5 \mathrm{P}_{\mathrm{hT}}\right)+0.5 \mathrm{M}_{\mathrm{hT}}\left(\mathrm{P}_{\mathrm{hh}}+0.5 \mathrm{P}_{\mathrm{hc}}+0.5 \mathrm{P}_{\mathrm{hT}}\right)$

where $\mathrm{P}$ is the proportion of that genotype in the population (representing the probability of the male being of that genotype).

The probability of a given mating occurring requires a knowledge of the probability that the mating is not the first for the male. It is very difficult to tell how many times a male mates in the wild, but an estimate can be made from discovering the number of times a female mates.

There are two ways in which this can be done without carrying out field work. Firstly, given that the dominance hierarchies of the alleles involved in morph coloration is known (from the work of Clarke \& Sheppard - see Appendix 1), it is possible to estimate roughly how many of the broods reported from wild females by Ford (1936) are likely to have been the result of multiple parentage. This method can only really be used to give a minimum proportion which have mated more than once due to the fact that females which mate with males whose genotype is either the same as, or have a genes which are recessive to those of, her other mate will have broods which only appear to have one father. This estimate is done in the final part of Appendix 4.

The second, and much more reliable way of assessing the number of times females mate in the wild is by counting the number of spermatophores present in their bursa copulatrix. The sprematophores are generally proteinaceous, and provide an accurate assessment of the number of times a female has mated successfully as only one is transferred per mating(Burns, 1968). A comprehensive review by Drummond (1984) details all the lepidopteran species for which spermatophore counts have been done. Within the genus Papilio, five species have been studied, and the results of those counts are shown in Table 7-5. 


\begin{tabular}{|c|c|c|c|c|c|c|}
\hline Species & $\begin{array}{c}\text { No. } \\
\text { investigated }\end{array}$ & $\begin{array}{c}\text { Mean no. } \\
\text { sperm's per } \\
\text { mated female }\end{array}$ & $\begin{array}{c}\text { Max no. } \\
\text { sperm's per } \\
\text { female }\end{array}$ & $\begin{array}{c}\text { \% } \\
\text { mated } \\
\text { once }\end{array}$ & $\begin{array}{c}\text { \% } \\
\text { mated } \\
\text { multiply }\end{array}$ & Reference \\
\hline Papilio glaucus & 220 & 1.16 & 3 & 84.1 & 15.0 & Pliske (1972) \\
\hline$“$ & 150 & 1.14 & 3 & 86.7 & 13.3 & Pliske (1973) \\
\hline$“$ & 84 & 1.75 & 5 & 46.4 & 53.6 & Burns (1968) \\
\hline$“$ & 200 & 1.54 & 3 & 51.5 & 46.5 & Makielski (1972) \\
\hline$“$ & 29 & 1.72 & 3 & 41.4 & 58.6 & Burns (1968) \\
\hline "“ & 92 & 1.73 & 3 & 37.0 & 63.0 & Burns (1968) \\
\hline Papilio palamedes & 32 & 1.47 & 3 & 65.5 & 34.4 & Pliske (1973) \\
\hline Papilio polyxenes & 171 & 1.33 & 3 & 67.3 & 30.4 & Lederhouse (1981) \\
\hline Papilio troilus & 358 & 1.23 & 4 & 79.9 & 19.6 & Pliske (1973) \\
\hline Papilio zelicaon & 97 & 1.23 & 3 & 75.3 & 19.5 & Sims (1979) \\
\hline " & 84 & 1.21 & 3 & 93.3 & 6.0 & Shields (1967) \\
\hline
\end{tabular}

Table 7-5 The results of spermatophore counts in five Papilio species, from Drummond (1984)

These results indicate that multiple mating is in fact quite common among Papilionids. In order to assess how common it

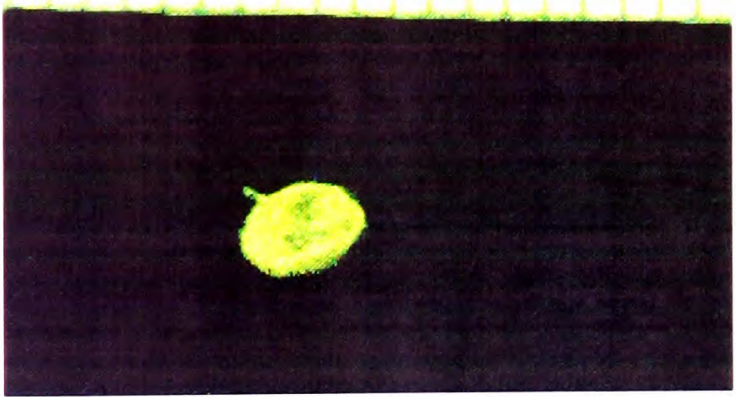

Figure 7-2 A large spermatophore from Papilio dardanus. The ruler above shows millimeters is in Papilio dardanus, the abdomens of wildcaught females from the collection by Cook et al. were dissected under water, and the number of spermatophores present counted. The spermatophores were easy to recognise being large, pale, and very hard. They were roughly classified as 'large' (>3mm diameter) 'small' (2$3 \mathrm{~mm})$, and 'very small' $(<2 \mathrm{~mm})$, as

spermatophore size is an indicator of whether or not they are likely to have come from a first, second, or third mating (Svärd \& Wicklund, 1986). Some spermatophores were categorised as 'very small' when it is possible they had been mostly used by the female, and that only the stem and part of the main body of the spermatophore remained. The results of the spermatophore count are shown in Table 7-6. 


\begin{tabular}{|c|c|c|c|c|}
\hline Morph & No. large sperm's & No. small sperm's & No. v. small sperm's & Total \\
\hline hippocoonides & 1 & 1 & 0 & 2 \\
\hline hippocoonides & 1 & 0 & 0 & 1 \\
\hline hippocoonides & 1 & 0 & 0 & 1 \\
\hline hippocoonides & 1 & 0 & 0 & 1 \\
\hline hippocoonides & 1 & 0 & 0 & 1 \\
\hline hippocoonides & 3 & 0 & 0 & 3 \\
\hline hippocoonides & 0 & 3 & 0 & 3 \\
\hline hippocoonides & 0 & 1 & 0 & 1 \\
\hline MEAN & 1 & 0.625 & $\mathbf{0}$ & 1.625 \\
\hline trimeni & 1 & 0 & 0 & 1 \\
\hline trimeni & 0 & 1 & 0 & 1 \\
\hline trimeni & 1 & 0 & 1 & 2 \\
\hline trimeni & 1 & 0 & 1 & 2 \\
\hline trimeni & 2 & 0 & 1 & 3 \\
\hline trimeni & 0 & 0 & 1 & 1 \\
\hline trimeni & 2 & 0 & 0 & 2 \\
\hline MEAN & 1 & 0.143 & 0.571 & 1.714 \\
\hline white lamborni & 2 & 0 & 0 & 2 \\
\hline white lamborni & 1 & 2 & 0 & 3 \\
\hline white lamborni & 1 & 1 & 0 & 2 \\
\hline MEAN & 1.333 & 1 & $\mathbf{0}$ & 2.333 \\
\hline yellow lamborni & 1 & 0 & 0 & 1 \\
\hline yellow lamborni & 1 & 1 & 0 & 2 \\
\hline yellow lamborni & 1 & 1 & 0 & 2 \\
\hline yellow lamborni & 1 & 1 & 0 & 2 \\
\hline MEAN & 1 & 0.75 & $\mathbf{0}$ & 1.75 \\
\hline OVERALL MEAN & 1.045 & 0.545 & 0.182 & 1.773 \\
\hline
\end{tabular}

Table 7-6 The results of spermatophore counts in Papilio dardanus females collected from Pemba by Cook et al.

This gives a mean number of matings by females to be 1.773 , which appears to be high for Papilio species (see Table 7-5). 40.9\% mated once, $40.9 \%$ mated twice, and $18.2 \%$ mated three times. $59 \%$ of the spermatophores were 'large', suggesting that they came from males who had not previously mated, $30.8 \%$ were 'small' suggesting that they came from males' second matings, and $10.3 \%$ were 'very small' suggesting that they may be from males' third matings. Since only second matings have been investigated experimentally (in Chapter 5), it seems best to assume that second and third matings can be treated the same. Therefore the percentages can be simplified to $40.9 \%$ mating once and $59.1 \%$ mating multiply for females. $59 \%$ of the spermatophores appear to be from a first mating for the males, and $41 \%$ are possibly from a subsequent mating (although the categorisation of spermatophores as from a first or subsequent mating is likely to be inaccurate). Since (to a good approximation) all females will be mated, and the sex ratio is unlikely to deviate from 50:50, either approximately the same number of males mate once and more than once as females, or only a few males mate at all, and most of them multiply. The spermatophore count does not represent either of these scenarios and so the spermatophore categorisation appears to be inaccurate - too many appear to 
be from a first mating. Since there is no evidence that most of the spermatophores are from subsequent matings, it seems best to assume that the number of males mating once and multiply is approximately equal to that of females, and hence the proportion that mate more than once is taken to be 0.59 in the model.

The differences between the morphs are interesting, although the dataset is too small to perform statistical tests. There is a suggestion that lamborni morphs are in fact mated more often than other morphs, and hippocoonides females have the lowest mean spermatophore count, contrary to the number of approaches to hippocoonides females recorded by Cook et al. (1994).

\section{Factor 3 Predation}

\section{Introduction}

Once the proportions of each genotype which will be present in the next generation have been calculated, the effect of predators on the offspring can be assessed. It has to be assumed, in the absence of evidence to the contrary, that predation at stages other than the adult is not influenced by the morph genotype of the individual. Hence predation is only considered at the adult stage.

\section{Method}

Huheey (1988) produced an equation describing the relationship between the frequency of a model and its mimic, and the probability that an individual mimic is attacked by a predator:

$$
P=1 /(p+n q)
$$

where $\mathrm{P}$ is the probability of an individual being attacked, $\mathrm{n}$ is the 'memory parameter' of the predator concerned (which depends on the palatabilities of the model and mimic), $p$ is the frequency of the mimic, and $q$ the frequency of the model.

He plots this equation in the form:

$$
(1 / P)-p=n q
$$

which is a straight line, and fits it to experimental data from Brower (1960 - experiments on starlings eating mealworms with or without distasteful quinine), Huheey (1980 - experiments on toads and treefrogs eating honeybees with or without stings), and Avery (1985 - two experiments on house finches eating seeds with or without the emetic chemical methiocarb). 
The whole area of the dynamics of mimicry is very little understood at present, and a comprehensive paper by Turner and Speed (1996) describes the problems very well. One of the most important aspects of mimicry is how the predator learns, and forgets, patterns. This will govern the frequency which the mimics have to reach with respect to their models in order to achieve protection, how close the mimicry has to be for it to be successful etc. Turner and Speed ran computer simulations of mimicry systems using many proposed learning and forgetting rules, and plotted the probabilities of a mimetic individual being eaten by the proposed predator as a function of the mimic frequency, producing graphs in the same form as the straight line predicted by Huheey above. These graphs vary in their shape depending on the learning and forgetting rules applied: out of 116 investigated possibilities, $61 \%$ were straight lines, or virtually indistinguishable from them, $32 \%$ were slightly concave, $5 \%$ were slightly convex, and $2 \%$ were irregular. Unfortunately, there is very little experimental evidence to help distinguish which rules are actually used by different predators. The only experiments which bear any light on the situation are those mentioned above which do not significantly deviate from a straight line.

Therefore, due to a lack of any other evidence, Huheey's model has been used in this simulation, with a variable memory parameter. This is the parameter which controls how long the memory of the distasteful model, once tried, remains with the predator, causing it to avoid models and mimics. It varies depending not only on the memory capacity and feeding strategy of the predator (which itself depends on the physiological state of the individual), but also on the relative toxicities of the model and mimic. Since no evidence exists on the learning and memory capacities of the major butterfly predators in Africa, this parameter remains unknown. Danaus chrysippus is not as efficient a sequesterer of cardiac glycosides as Danaus plexippus (Rothschild et al. 1975), and so the learning parameter might be expected to be relatively low. Brower (1960) found $n=11$ for starlings feeding on quinine models (which are also not particularly distasteful). This parameter will be varied and discussed with the results in the 'mathematical model' section of this chapter.

Another factor involved with predation is the number of individual predators feeding on the population. Huheey's model predicts the outcome of an individual encounter, but the number of encounters itself is a further variable. This is unpredictable, but may cause very large differences between the morph balances in different populations of Papilio dardanus. Therefore this parameter will also be varied, and discussed in the 'model' section of the chapter.

Using this method, the proportion of each morph surviving predation can be calculated, and this can be multiplied by the proportion of the morph in the population. This figure is the proportion of adults of each morph in the second generation surviving to breed. By repeating the calculations 
using these figures, the changes in morph frequency in the population under various conditions can be modelled.

\section{Results}

Chapter 6 indicated that the mimetic female forms of Papilio dardanus were indeed palatable, Batesian mimics. This means that the non-mimetic forms will have no protection from predators, and the mimetic ones will have protection which varies with the memory capacity and feeding strategy of the predators (affecting the parameter ' $n$ ').

$\mathrm{p}$ and $\mathrm{q}$, the frequencies of the model (as assessed by the collector) and mimic, can be taken from Ford (1936) for the races meseres and polytrophus, where he cites data from random collections by van Someren in 1923 and Wiggins some time before 1910. These are shown in Table 7-7.

\begin{tabular}{|c|c|c|c|c|}
\hline Race & Morph & Number & Model & Number \\
\hline Meseres & hippocoonides & 70 & Amauris niavius & 104 \\
\hline & $\begin{array}{c}\text { planemoides, } \\
\text { carpenteri }\end{array}$ & 25 & $\begin{array}{c}\text { Bematistes poggei, \% Bematistes macarista, } \\
\text { Amauris alciope (\&form aurivilli) }\end{array}$ & 781 \\
\hline & cenea & 8 & Amauris echeria, Amauris albimaculata & 232 \\
\hline & niobe & 8 & $\begin{array}{c}\text { Bematistes tellus, Amauris jodutta, Amauris } \\
\text { althoffi }\end{array}$ & 832 \\
\hline Polytrophus & cenea & 65 & $\begin{array}{c}\text { Amauris echeria, Amauris albimaculata, } \\
\text { Pseudacrea leucretia }\end{array}$ & 32 \\
\hline & hippocoonides & 53 & Amauris niavius & 0 \\
\hline & salaami & 15 & NONE & 0 \\
\hline
\end{tabular}

Table 7-7 Table showing the proportions of model and mimic reported by Ford (1936) for races polytrophus and meseres

Of course, the non-mimetic forms (such as the andromorphic trimeni) will have no models at all, so will gain no protection from predators.

These parameters (the relative proportions of model and mimic, and the 'memory' parameter affected by the relative palatabilities of model and mimic) will be varied and the results discussed in the 'mathematical model' section of this chapter which follows.

\section{The mathematical model}

\section{Introduction}

The factors listed above were all incorporated into a model of the population in the form of a computer program written in C (see Appendix 6 for a program listing). The model simulated the change in morph proportions through successive generations under the conditions imposed. Each 
parameter which has been assessed above was entered into the model, leaving the unknown value of Huheey's 'memory parameter' and the rate of predation to be varied.

\section{Method}

The first population to be modelled was the race polytrophus, with the values of the ratio of model to mimic as reported in Ford (1936) and shown in Table 7-7. Ford gave values for only three morphs (although trophonius is reported as being present in the race - see Appendix 1), two mimetic (although he reported no models of hippocoonides, so the ratio of model:mimic was taken to be 5:53) and one he reports to be non mimetic (he calls it salaami, although it no doubt equates to the form poultoni as described in Appendix 1 as present in race polytrophus). The proportions reported for these three morphs in Table 7-7 were therefore entered as the starting proportions, and the values of the memory and predation pressure parameters varied in an attempt to find a stable balance of the morphs.

The second population to be modelled was that from Pemba, Tanzania - studied by Cook et al. (1994). Unfortunately for this population the ratio of the models to the mimics was not assessed accurately, although the model of the rarer mimic (trophonius) was reported to be rare also (pers. comm.), and so the ratios were set to 1:1 for each of the two mimetic morphs (the non mimetic morph trimeni had no model, of course). Once the optimum parameters for stability had been found, then the ability of the different morphs to invade a monomorphic or dimorphic starting population were investigated.

\section{Results}

In the polytrophus race, it is clear from looking at the starting proportions that salaami is never going to be maintained in the population under the conditions imposed by the model. The mate choice favours the most common morphs, and the predator pressure favours the mimetic morphs. Therefore a non-mimetic, rare morph is always going to be excluded from the population whatever the variable parameters are set to. This is shown by the model in Figure 7-2. 


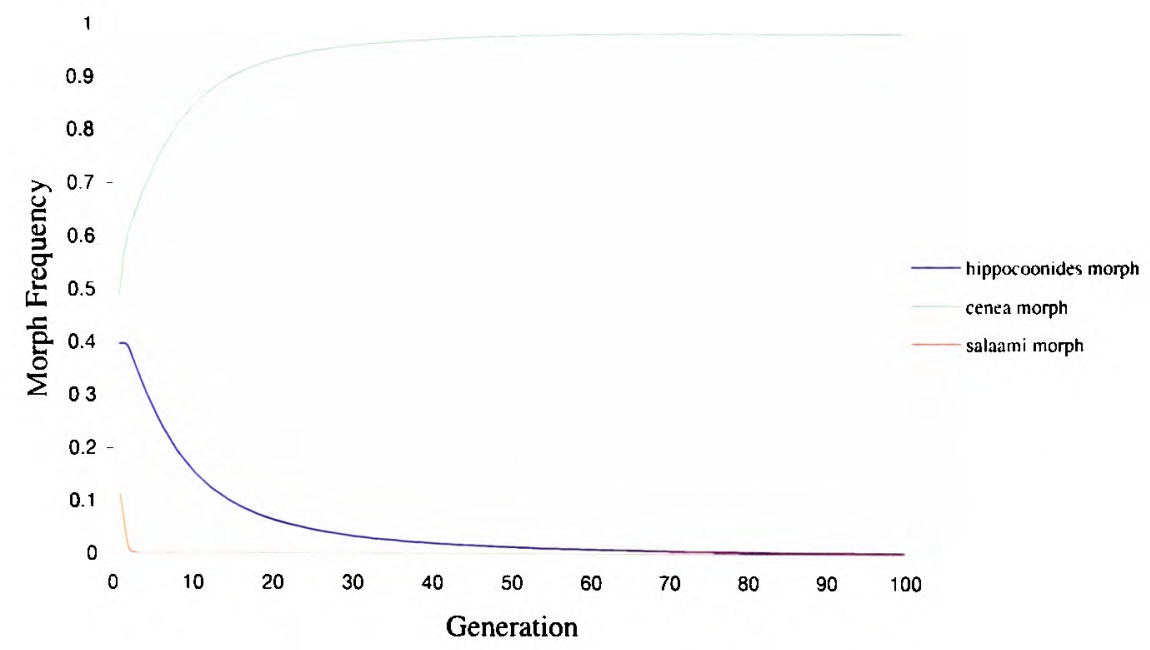

Figure 7-3 Mathematical model of the polytrophus population showing that the rare, non-mimetic morph (salaami) cannot be maintained in the population (parameters: probability second mating $=0.59$, probability choosing same morph twice $=0.75$, probability encounter predator before mating $=0.95$, memory of predator $=40$, abundance of models and mimics as in Table $7-7$ ).

The hippocoonides morph declines as it has much less protection from predators then cenea, and also starts at a lower frequency, and thus is slightly disadvantaged with respect to mate choice.

In the race meseres, Ford (1936) reported no non-mimetic morphs. However, using the figures from Table 7-7 (and removing niobe from the population, since the model is only written to simulate three morphs in a population) it is still not possible to balance the morphs in the population (see Figure 7-4). This is because the only variables are the encounter rate and memory of the predators, and this will only alter the amount of advantage in natural selection of the most protected morph. Since the sexual selection pressure is so strong, it is impossible to counter the effect on the most common morph, even though it is the least protected in terms of the initial ratio of model to mimic, and its increasing frequency decreases the ratio of model to mimic.

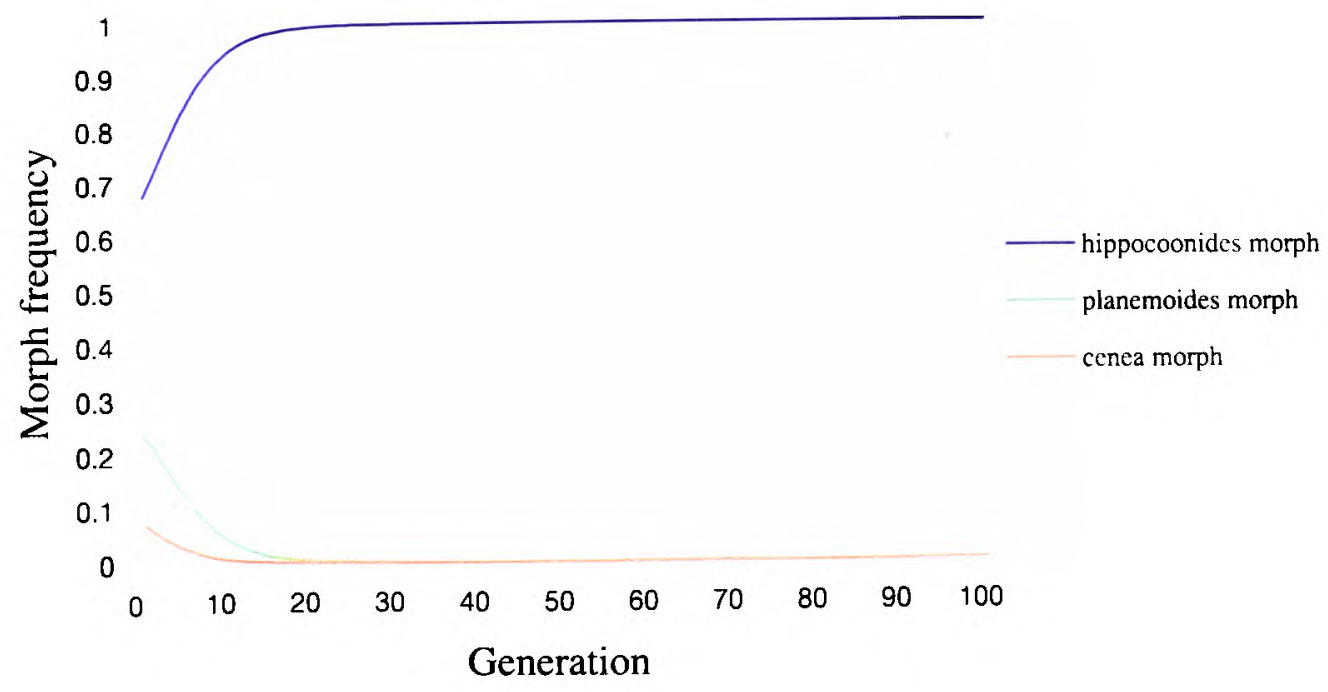

Figure 7-4 Mathematical model of the meseres population showing that the rare morphs cannot be balanced in the population (parameters: probability second mating $=0.59$, probability choosing same morph twice $=0.75$, probability encounter predator before mating $=0.95$, memory of predator $=40$, abundance of models and mimics as in Table 7-7). 
In the Pemba population, the relatively rare andromorph will again be at a disadvantage both in natural and sexual selection according to this model, and hence it would be expected to be eliminated from the population. With the strong sexual selection advantage conferred upon the most common morph (in this case, hippocoonides), it is also not possible to keep the rare trophonius in the population, even by reducing the probability of choosing the same morph twice to 0.5 (see Figure 7-5).

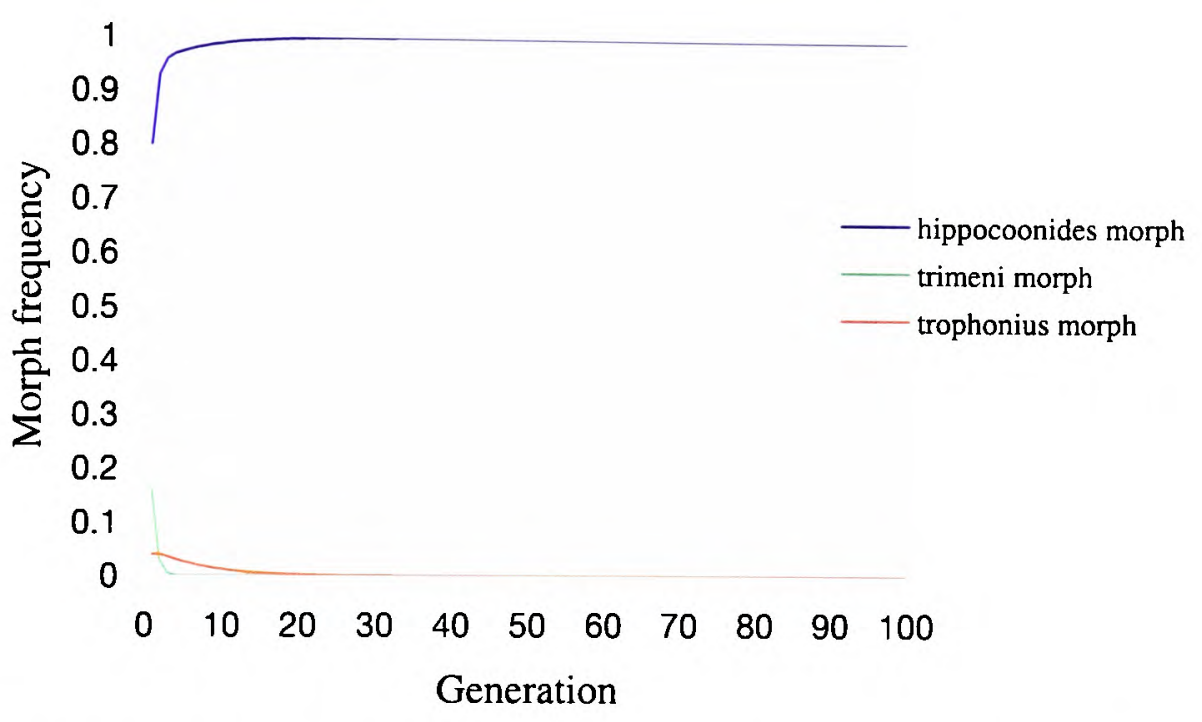

Figure 7-5 Mathematical model of the Pemba population, showing that trimeni and trophonius can never be maintained under this model (parameters: probability second mating $=0.59$, probability choosing same morph twice $=0.5$, probability encounter predator before mating $=0.9$, memory of predator $=1000$, initial frequencies of each morph as in Appendix 5 , initial ratio model: mimic for hippocoonides $=1$, for trophonius $=1000$ ).

Even by decreasing the advantages of hippocoonides by increasing massively the protection of trophonius (by increasing the ratio of models to mimics) and the memory of the predators (to strengthen the protection to trophonius), hippocoonides is too common at the outset to be maintained.

It is possible to investigate the evolution of the different morphs by running the simulation with a monomorphic starting population (and a tiny proportion of new mutant morphs). The initial starting population was first taken to be monomorphic andromorphs. With different parameters, the final outcome of the population can be very different. In some cases, one or other of the mimetic morphs goes to fixation in the population (although the dynamics of this can be very interesting, as in Figure 7-6). 


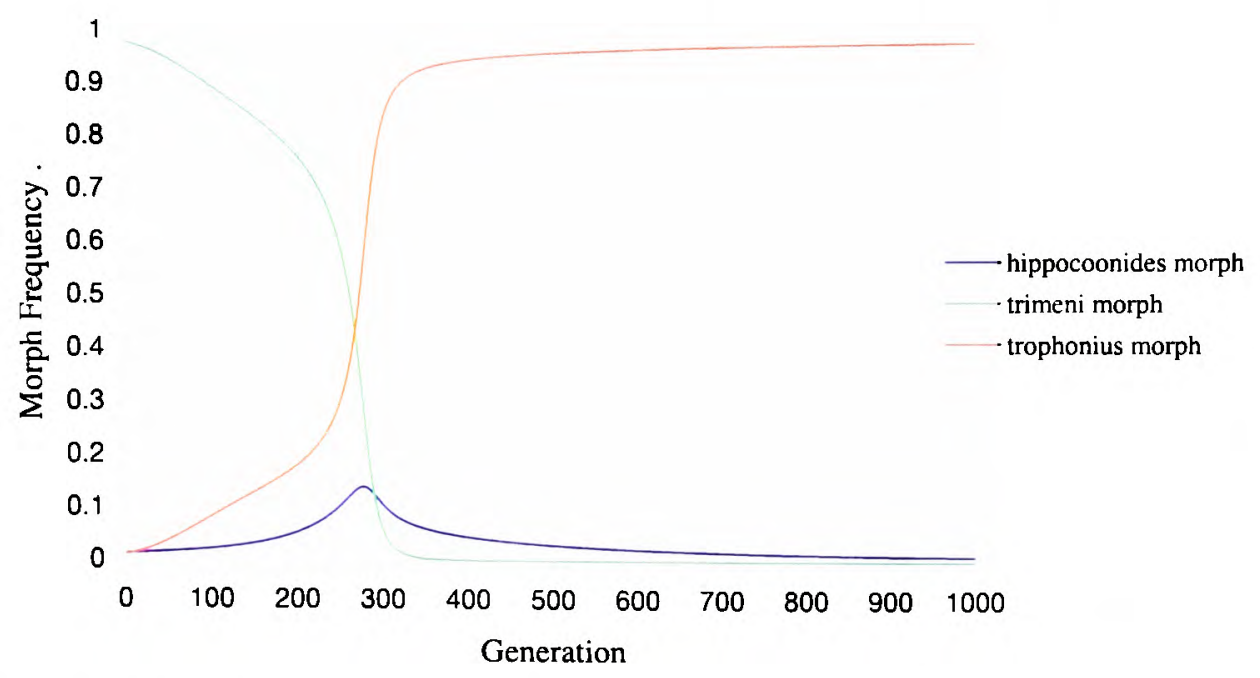

Figure 7-6 Mathematical model of invasion of hippocoonides and trophonius morphs in a monomorphic population of trimeni showing one of the mimetic morphs going to fixation (parameters: probability second mating $=0.59$, probability choosing same morph twice $=0.5$, probability encounter predator before mating $=0.3$, memory of predator $=11$, initial frequency trimeni $=0.98$, frequencies of other morphs $=0.01$, initial ratio model:mimic for hippocoonides $=4$, for trophonius $=2.5$ ).

Here, the andromorphs immediately start to decline, due to predator pressure, and their place is taken by both trophonius and hipppocoonides. The dominant trophonius, however, does slightly better than hippocoonides initially, and as the decline of the andromorphs starts to accelerate (as they lose the sexual selection advantage), trophonius starts to increase rapidly, and hippocoonides again goes into decline as trophonius has an advantage in both natural and sexual selection.

Another alternative is that the two invasive mimetic morphs come to balance, whilst eliminating the non-mimetic andromorph (see Figure 7-7 and Figure 7-8).

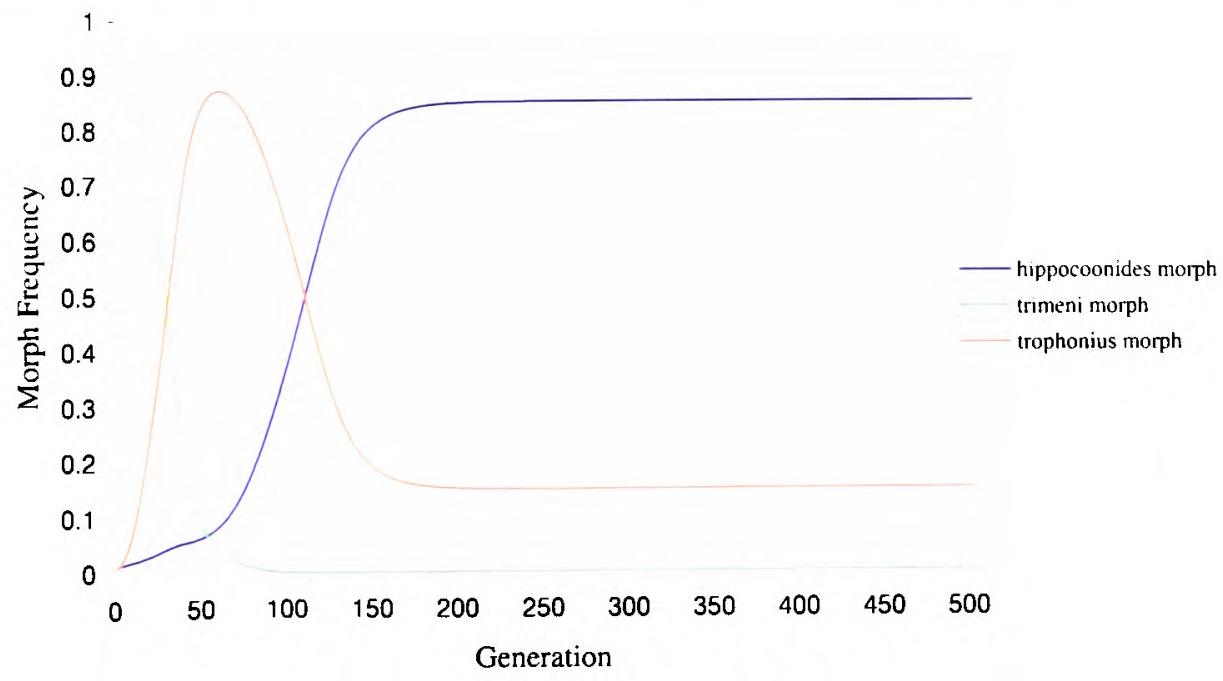

Figure 7.7 Mathematical model of invasion of hippocoonides and trophonius morphs in a monomorphic population of trimeni showing hippocoonides and trophonius coming to balance (parameters: probability second mating $=0.59$, probability choosing same morph twice $=0.5$, probability encounter predator before mating $=0.5$, memory of predator $=11$, initial frequency trimeni $=$ 0.98 , frequencies of other morphs $=0.01$, initial ratio model: mimic for hippocoonides $=4$, for trophonius $=2.2$ ). 


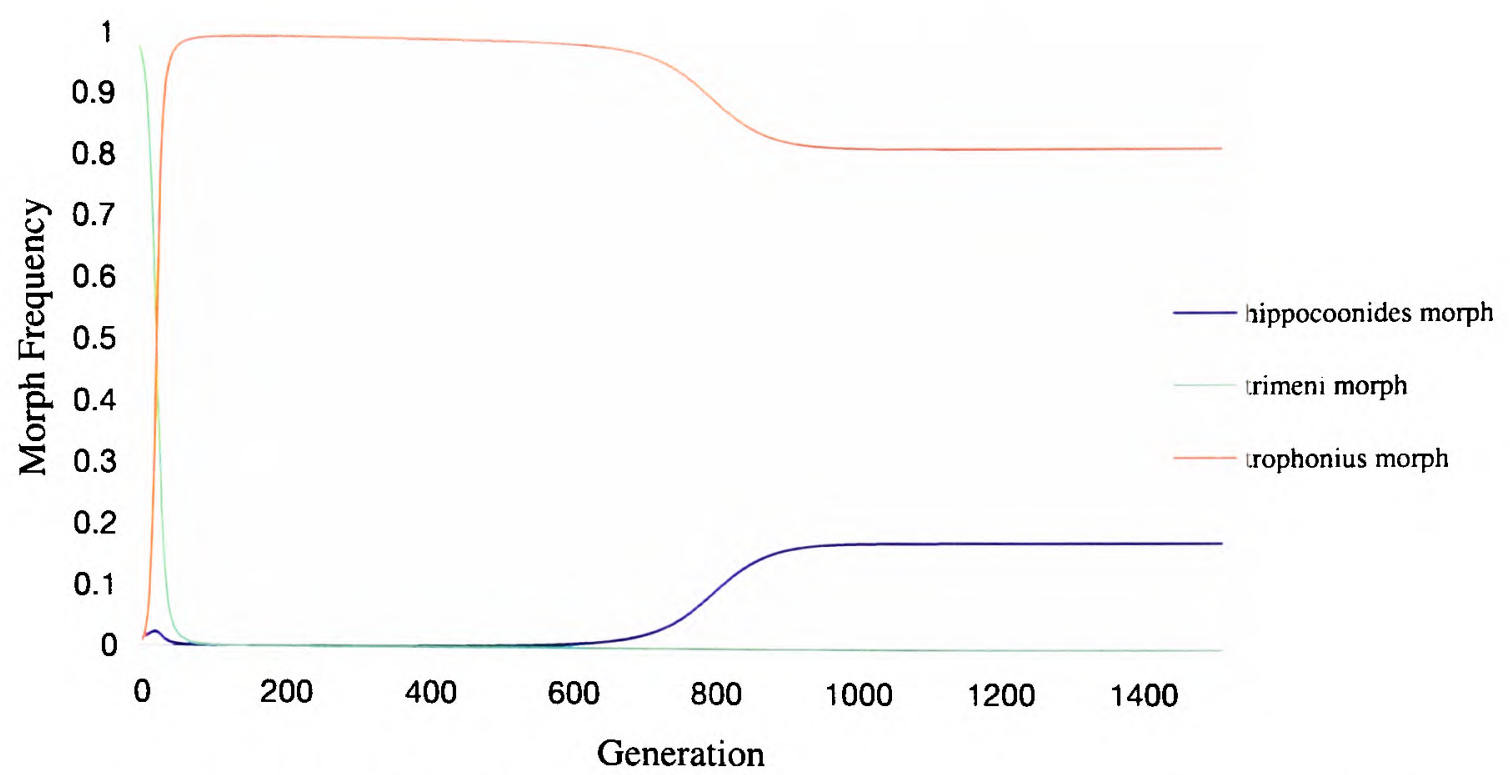

Figure 7-8 Mathematical model of invasion of hippocoonides and trophonius morphs in a monomorphic population of trimeni showing hippocoonides and trophonius coming to balance (parameters: probability second mating $=0.59$, probability choosing same morph twice $=0.5$, probability encounter predator before mating $=0.5$, memory of predator $=11$, initial frequency trimeni $=$ 0.98 , frequencies of other morphs $=0.01$, initial ratio model:mimic for hippocoonides $=4$, for trophonius $=6$ ).

This alteration of the fortunes of each mimetic morphs is achieved simply by slight increases or decreases in the initial ratio of model to mimic (which, of course, varies as the morph frequencies vary), to which the mathematical model is very sensitive, and this demonstrates why the morph balance changes so rapidly across areas where the balance of the different models changes.

By altering the parameters, however, it is possible to balance the factors ending in a balanced polymorphism of all three morphs, with varying proportions of each morph (see Figure 7-9 and Figure 7-10).

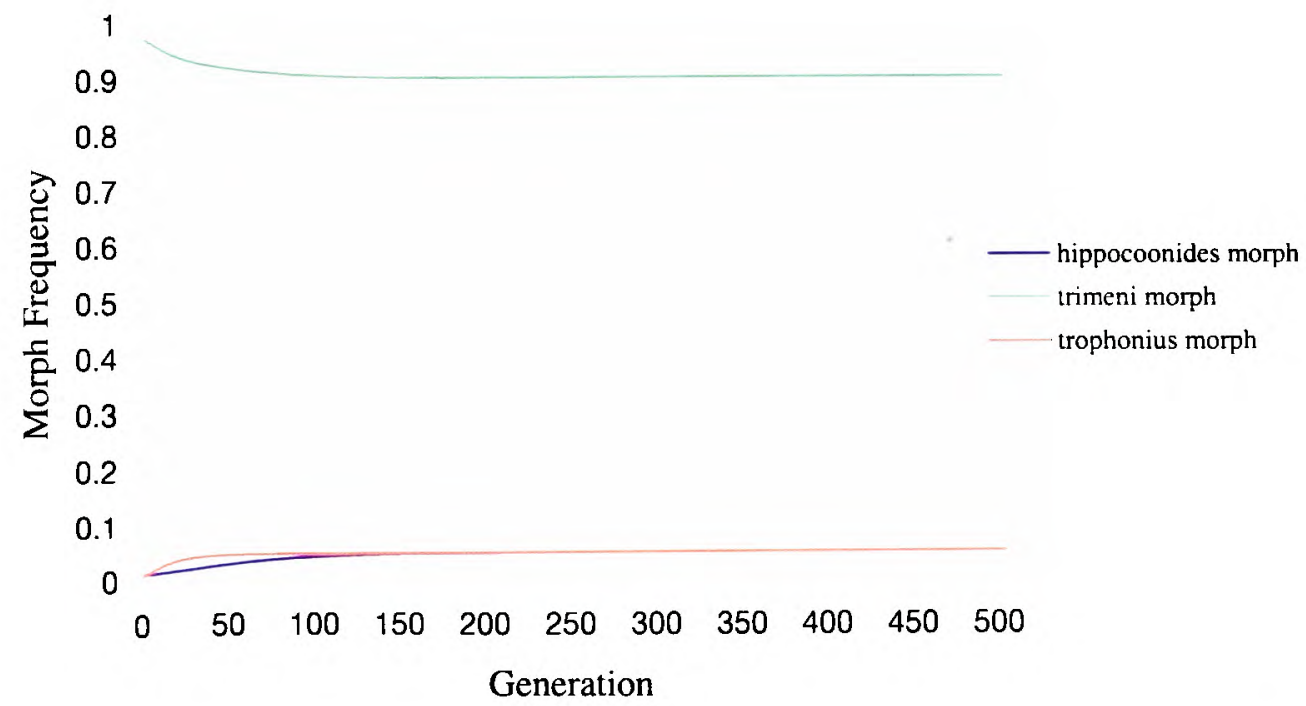

Figure 7-9 Mathematical model of the invasion of hippocoonides and trophonius morphs into an andromorphic population resulting in a balanced situation (parameters: probability second mating $=0.59$, probability choosing same morph twice $=0.5$, probability encounter predator before mating $=0.5$, memory of predator $=3$, initial frequency trimeni $=0.98$, frequencies of other morphs $=0.01$, initial ratio model: mimic for hippocoonides $=1$, for trophonius $=1$ ). 


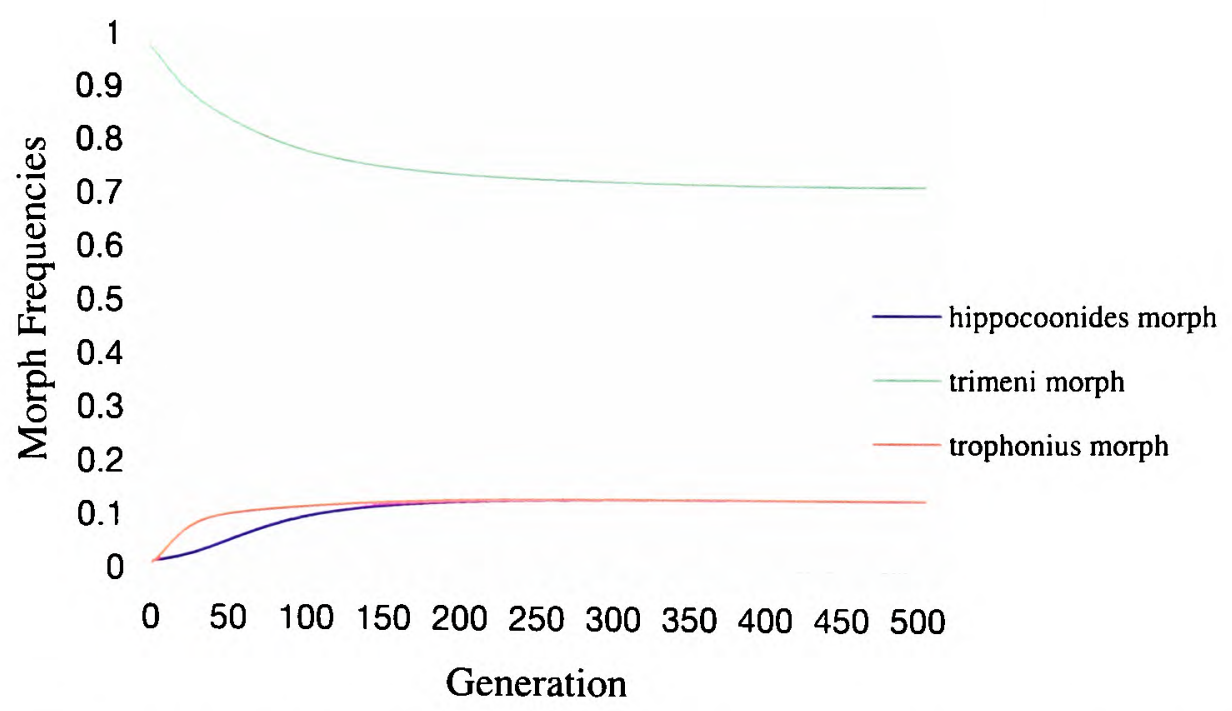

Figure 7.10 Mathematical model of the invasion of hippocoonides and trophonius morphs into an andromorphic population resulting in a different polymorphism (parameters: probability second mating $=0.59$, probability choosing same morph twice $=$ 0.5 , probability encounter predator before mating $=0.5$, memory of predator $=5$, initial frequency trimeni $=0.98$, frequencies of other morphs $=0.01$, initial ratio model: mimic for hippocoonides $=1$, for trophonius $=1$ ).

This change in the population structure was achieved by altering the memory of the predator (which is affected not only by the predators, but also by the relative distastefulness of the models and mimics). It shows how andromorphs may be maintained in a balanced polymorphism as long as they remain above a certain threshold level. If they fall below about $50 \%$ in the population, they lose their sexual selection advantage, and are rapidly eliminated from the population.

If the population does not include andromorphs there is, of course, no way in which they can invade, and even if the starting population is dimorphic, with a high proportion of andromorphs to mimetic morphs, the added advantage of the mimicry leads to the andromorphs becoming rapidly extinct (see Figure 7-11). Therefore in the current model, the only way in which andromorphs can be maintained in the population is for them to be the ancestral, monomorphic condition and for them to remain at a relatively high proportion in the population when mimetic morphs invade. 


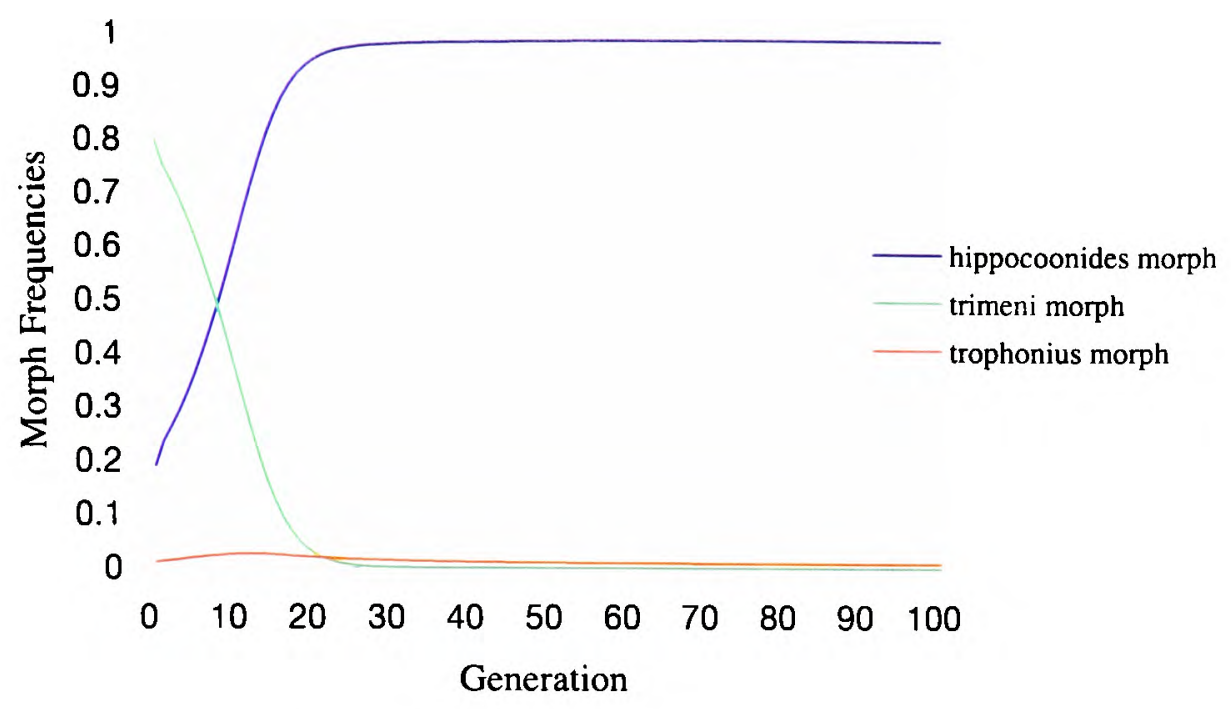

Figure 7-11 Mathematical model showing the demise of the andromorphs in an initially dimorphic population with a high proportion of andromorphs (parameters: probability second mating $=0.59$, probability choosing same morph twice $=0.5$, probability encounter predator before mating $=0.5$, memory of predator $=5$, initial frequency trimeni $=0.80$, frequency hippocoonides $=0.19$, freqency trophonius $=0.01$, ratio model mimic for hippocoonides $=1$, for trophonius $=1$ ).

Cook et al. (1994) and Vane Wright (1984) suggest that the andromorphs may have an advantage in sexual selection, although Vane Wright's 'pseudosexual selection' hypothesis maintains that this advantage is through increased matings due to males approaching andromorphs as if they were males (for aggressive encounters), whilst Cook et al. suggest that the andromorphs are approached less often, and that this lack of sexual harrassment provides the andromorphs with an advantage. Although the naïve males in behavioural experiments in Chapter 5 appeared to mate at random, no andromorphs were available to test the either hypothesis, and so a preference or avoidance of andromorphs cannot be ruled out in this species.

It is possible to alter the mathematical model to alter the probability of a male mating with each morph on his first mating. This allows the pseudosexual selection hypothesis to be investigated. Cook et al.'s sexual harrassment hypothesis effectively amounts to the same thing - in reality the andromorphs are not being mated more frequently (as the model suggests), but the result of them leaving more offspring is the same.

If Cook et al.'s frequencies of approaches to each morph are used as the frequencies of initial matings (although the inverse is taken, as they assume increased approach to be detrimental), it is not possible to achieve a balanced polymorphism of all three morphs (by altering the predation parameters), but it is easy to balance hippocoonides and trophonius (see Figure 7-12). 


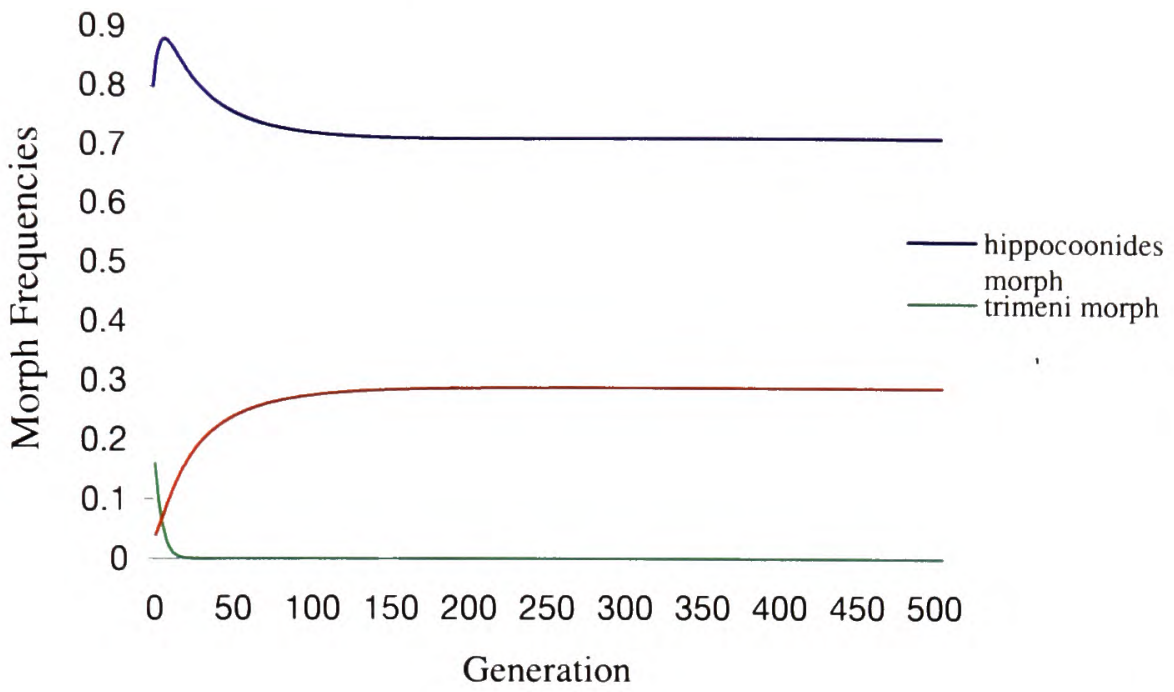

Figure 7-12 Mathematical model using initial mating frequencies from Cook et al. (1994), showing that andromorphs still cannot be maintained (parameters: probability second mating $=0.59$, probability choosing same morph twice $=0.5$, probability encounter predator before mating $=0.5$, memory of predator $=5$, initial frequencies of morphs, and initial mating frequencies as in Appendix 5 , ratio model: mimic for hippocoonides $=1$, for trophonius $=1$ ).

By altering these initial mating frequencies, it is almost possible to achieve the balanced polymorphism seen in the wild population. However, due to the strong frequency-dependent mate choice (and the frequency-dependent predator pressure), this balance is on a knife edge, and any slight perturbation causes it to break down very rapidly (see Figure 7-13).

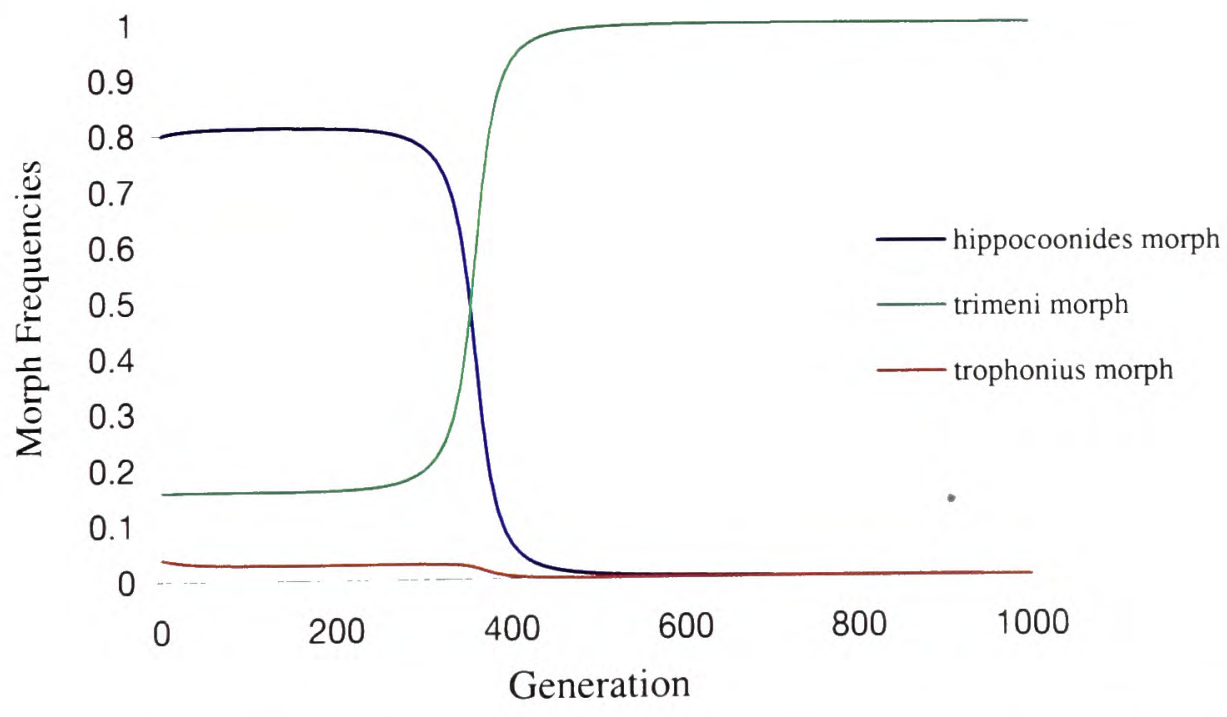

Figure 7-13 Mathematical model demonstrating a knife-edge polymorphic balance, achieved by altering initial mating frequencies and predator parameters (parameters: probability second mating $=0.59$, probability choosing same morph twice $=0.5$, probability encounter predator before mating $=0.5$, memory of predator $=5$, initial frequencies of morphs as in Appendix 5 , initial mating frequency for hippocoonides $=0.45$, for trimeni $=1$, for trophonius $=0.722$, ratio model : mimic for hippocoonides $=1.8587$, for trophonius $=1.8587$ ) . 


\section{Discussion}

The model clearly illustrates that under the conditions imposed by the factors discussed earlier in the chapter, the low proportions of non-mimetic morphs reported from some populations of Papilio dardanus cannot be stable. Therefore these populations are either not balanced, and are undergoing the loss of non-mimetic morphs, or there are other factors acting which have not been taken into account. The first of these explanations seems unlikely as, although the population balance may well shift through time, the model shows a very rapid extinction of non-mimetic forms, and the forms reported over 50 years ago by Ford (1936) and others are still present in the populations, several hundred generations later.

It seems, therefore, that pressures other than those considered in this thesis may be acting. In the mate choice experiments it was not possible to obtain enough non-mimetic morphs to carry out choice tests using them. This is unfortunate, as it has therefore not been possible to test the 'pseudosexual selection' hypothesis (Vane Wright, 1984) in which andromorphic females are approached more often by males as they are mistaken for males and thus approached aggressively or the opposite view put forward by Cook et al. (1994), who suggested that excess male attention may be a handicap to females. Both theories suggest an advantage to andromorphs through a varying initial approach rate, which has been shown in this model to be unlikely to hold the polymorphism in a stable balance if there is a learning effect in the male mate choice due to the magnifying effect this has on small perturbations in the proportions of the morphs. Behavioural observations (see Chapter 5) suggest that males do approach other males more often than females, running counter to the observations of Cook et al. (1994) who found trimeni females to be approached less often than hippocoonides females by wild males.

By altering the model to take into account varying frequencies of initial matings for each morph it is possible to obtain a balanced polymorphism as seen in the wild populations, but the strong frequency-dependent selection pressures in both natural and sexual selection make this balance very easily perturbed, and therefore the wild populations might be expected to show morph fluctuations. Since no regular morph frequency assessments have been reported for the species, it is not possible to say whether or not this does actually occur. However, the model does illustrate how sensitive the morph frequencies are to changes in the ratio of model to mimic, and this is seen in wild populations where morph frequencies change to match those of the models (Ford, 1936).

The model shows how it is possible under the conditions imposed for a monomorphic nonmimetic population to be invaded by mimetic morphs, but for the non-mimetic morphs still to be maintained (as long as they do not fall below a certain threshold of frequency). This may be similar to the conditions seen in race antinorii, where the andromorphs are very frequent in the population, and 
the mimetic morphs are kept relatively rare. It is, however, much more difficult for andromorphs to invade a mimetic population - without the added advantage of differing rates of initial matings it is impossible, and the advantage which would need to be conferred to allow it would be very great indeed. It therefore seems more likely, on the basis of this population model, that the andromorphic females are ancestral to the mimetic morphs.

\section{Conclusions}

This thesis has examined the ways in which butterflies receive and perceive visual signals. In the case of Argynnis paphia, the males appear to show a preference for orange when choosing mates and flowers which is not related to their signal reception. This implies that the preference is due to their perception of the colour - either they have evolved a preference which allows them to recognise the orange females as mates which also has the effect of causing them to prefer orange flowers, or they have an unselected 'hidden preference', which might even have been exploited subsequently by the orange female morph. It has not been possible to distinguish between these two possibilities, although mate choice work carried out in a population which was dominated by the green female morph may be illuminating. It would also be necessary to carry out experiments to determine whether or not the males are learning the preference for orange females.

The work on Papilio dardanus has brought to light several behaviour patterns which have not been reported before. Firstly, the butterflies have been shown to have a preference for blue flowers over red and yellow (a pattern which is seen in other Papilionids: Ilse, 1928; Ilse \& Vaidya, 1956; Swihart, 1970). The lack of interest in red flowers can be explained by their lack of spectral sensitivity in this area of the spectrum, but this does not explain their preference for blue over yellow. Further experiments suggested that colour may not be the most important factor for the butterflies as their preference could change dramatically to red if the flowers were left unpainted. What caused this switch in behaviour has still to be discovered. It was also shown that individual butterflies tended to be $90 \%$ constant to a particular colour when they were rewarded for visiting that colour (but some data suggested that they tended to try other colours if their first choice did not provide food). For the first time it was demonstrated that the presence of other butterflies feeding could influence the behaviour of individuals, causing them to choose flowers which were spatially close to that on which another butterfly was feeding, regardless of its colour.

It was not possible to test whether or not male Papilio dardanus would show a preference for blue female morphs, as they could not be made to react to models or altered females. However, naïve males appeared to mate at random when first encountering females, not showing any colour preferences at all. When mated males were subsequently given the same choice of females, however, 
they tended to prefer females of the morph with which they had already successfully mated. This apparent colour constancy and rapid learning is similar to that seen in the flower choice experiments, but the initial preference is lacking.

The model of random mating followed by rapid learning from a first mating would, in general, explain the results of Cook et al. (1994) who found that male Papilio dardanus showed a significant preference for the most common morph. The actual data acquired by Cook et al. cannot be explained simply by such a model of behaviour, but discrepancies could be due to the pair-wise presentation methods employed and the self-selecting nature of the experimental butterfly group.

It has not been possible to assess accurately the palatability of Papilio dardanus relative to its distasteful models, although starlings appeared to find the species entirely palatable (see Chapter 6). An indirect measure of the palatability, the morphology of the butterflies (also assessed in Chapter 6), suggests that Papilio dardanus is indeed a Batesian mimic, as the centre of mass of the males is in the position expected for palatable species. The females, however, were shown to have a centre of mass further from the wingbase than both males and both sexes of Danaus chrysippus, which would cause them to be more prone to capture by birds than males (Srygley \& Dudley, 1993), and it is possible that this has been a factor causing female-limited mimicry in this species. This was not true of Danaus chrysippus, where males and females showed similar positions of the centre of mass. In addition, Srygley (1994) found that mimetic species tended to have centres of mass placed further back from the wingbase than non-mimetic species. Taken together, this evidence suggests that the position of the centre of mass, and its effect on the agility of a butterfly in flight, may be a factor affecting the evolution of mimicry in a species, or (where the egg load of the females causes their centre of mass to be placed further back) in only the females of a species.

When this mate choice and palatability information is assessed by means of creating a mathematical model of the balance of the morphs in a population, it can be seen that for the model to explain the current morph balances in different populations arf initial mating advantage for nonmimetic morphs has to be introduced. This could be investigated by continuing mate choice experiments similar to those in Chapter 5, but using andromorphic and non-mimetic females as well as those already studied. It appears likely that andromorphic females are gaining an advantage in sexual selection, although there are two opposing hypotheses to explain this (Cook et al., 1994 and Vane Wright, 1984). From behavioural observations in the flight cage (see Chapter 5) it appears likely that the males would approach andromorphic females more often than mimetic females, in accordance with the pseudosexual selection theory (Vane Wright, 1984), although this has yet to be tested. Some of the morphs described as non-mimetic which are not andromorphic may well in fact be mimetic, at least to some degree, of unidentified butterflies, which would give them some protection from 
predators, and the presence of a pyrazine odour is likely to reinforce this protection (Rowe \& Guilford, 1996).

The model also suggests that the andromorphic females are most likely to be ancestral to the mimetic females, as the frequency-dependent sexual selection makes it very difficult for them to invade a mimetic population. A molecular genetic approach, however, is likely to shed further light on the evolution of the species and its morphs.

\section{Acknowledgements}

I am very grateful to John Alden for help with C programming and to Yan Wong for help with modelling techniques, and to both for help in creating the Papilio dardanus population genetics Website. I would also like to thank Nigel Venters for discussions about wild populations of Papilio dardanus.

\section{$\underline{\text { References }}$}

Avery, M.L. 1985. Applications of mimicry theory to bird damage control. J. Wildl. Mgmt. 49, 116121.

Brower, J.V.Z. 1960. Experimental studies of mimicry. Part IV. The reactions of starlings to different proportions of models and mimics. Am. Nat. 94, 271-282.

Burns, J.M. 1968. Mating frequency in natural populations of skippers and butterflies as determined by spermatophore counts. Proc. Nat. Acad. Sci. 61, 852-859.

Cook, S.E., Vernon, J.G., Bateson, M., Guilford, T. 1994. Mate choice in the polymorphic African swallowtail butterfly, Papilio dardanus: male-like females may avoid sexual harassment. Anim. Behav. 47, 389-397.

Drummond, B.A. 1984. Multiple mating and sperm competition in the Lepidoptera. In Sperm competition and the evolution of animal mating systems (ed. Şmith, R.L.). Academic Press, London. P291-370.

Ford, E.B. 1936. The genetics of Papilio dardanus Brown (Lep.) Trans. R. Ent. Soc. London 85, 435-465.

Huheey, J.E. 1980. Studies in warning coloration and mimicry. VIIl. Further evidence for a frequency-dependent model of predation. J. Herpetol. 14, 223-230.

Huheey, J.E. 1988. Mathematical models of mimicry. Am. Nat. 131, S22-S41.

Ilse, D. 1928. Über den Farbensinn der Tagfalter. Z. f. vergl. Physiologie Bd. 8, 658-692.

Ilse, D. \& Vaidya, V.G. 1956. Spontaneous feeding response to colours in Papilio demoleus L.. Proc. Indian Acad. Sci., Sect. B 43, 23-31. 
Lederhouse, R.C. 1981. The effect of male mating frequency on egg fertility in the black swallowtail, Papilio polyxenes asterius (Papilionidae). J. Lepid. Soc. 35, 266-277.

Makielski, S.K. 1972. Polymorphism in Papilio glaucus L. (Papilionidae): maintenance of the female ancestral form. J. Lepid. Soc. 26, 109-111.

Pliske, T.E. 1972. Sexual selection and dimorphism in female tiger swallowtails, Papilio glaucus L. (Lepidoptera: Papilionidae): A reappraisal. Ann. Entomol. Soc. Am. 65, 1267-1270.

Pliske, T.E. 1973. Factors determining mating frequencies in some New World butterflies and skippers. Ann. Entomol. Soc. Am. 66, 146-169.

Rothschild, M., Von Euw, J., Reichstein, T., Smith, D.A.S., \& Pierre, J. 1975. Cardenolide storage in Danaus chrysippus with additional notes on D. plexippus. Proc. R. Soc. B 190, 1-31.

Rowe, C. \& Guilford, T. 1996. Hidden colour aversions in domestic chicks triggered by pyrazine odours of insect warning displays. Nature 383, 520-522.

Shields, O. 1967. Hilltopping: an ecological study of summit congregation behaviour of butterflies on a southern California hill. J. Res. Lepid. 6, 69-178.

Sims, S.R. 1979. Aspects of mating frequency and reproductive maturity in Papilio zelicaon. Am. Midl. Nat. 102, 36-50.

Srygley, R.B. 1994. Locomotor mimicry in butterflies? The associations of positions of centres of mass among groups of mimetic, profitable prey. Phil. Trans. R. Soc. Lond. B 343, 145-155.

Srygley, R.B. \& Dudley, R. 1993. Correlations of the position of center of body mass with butterfly escape tactics. J. Exp. Biol. 174, 155-166.

Svärd, L. \& Wicklund, C. 1986. Different ejaculate delivery strategies in first versus subsequent matings in the swallowtail butterfly, Papilio machaon L. Behav. Ecol. Sociobiol. 18, 325-330.

Swihart, S.L. 1970. The neural basis of colour vision in the butterfly Papilio troilus. J. Insect Physiol. 16, 1623-1636.

Turner, J.R.G. \& Speed, M.P. 1996. Learning and memory in mimicry. I. Simulations of laboratory experiments. Phil. Trans. R. Soc. Lond. B 351, 1157-1170.

Vane Wright, R.I. 1984. The role of pseudosexual selection in the evolution of butterfly colour patterns. In The Biology of Butterflies (ed. Vane Wright, R.I. \& Ackery, P.R.) Princeton, USA. 


\section{Appendix 1}

\section{The races and morphs of Papilio dardanus}

\section{Introduction}

Papilio dardanus (Brown), the Mocker Swallowtail, is widespread across sub-Saharan Africa, and about 13 geographical races have been described. This is a brief summary of the main races and morphs described for Papilio dardanus by Clarke \& Sheppard (1959, 1960a, 1960b, 1962) and Ford (1936). The genetics of Papilio dardanus have been studied extensively by Sir Cyril Clarke and the late Philip Sheppard by forming hybrids between races. They found that the mimetic patterns were controlled by closely linked genes at a single locus and that they had a distinct order of dominance. The dominance is almost always complete, preventing intermediate forms from arising. There are, however, modifier genes on other chromosomes which perfect the mimicry. These are not present in the monomorphic races meriones and humbloti, and therefore hybrids between these races and mainland polymorphic races results in imperfect mimetic patterns (Clarke \& Sheppard 1963) The morphs are represented by a single allele notation, and these are listed together with the description of each morph. Further information and colour photographs of some of the morphs are available on the Web site:

http://www.linacre.ox.ac.uk/research/dardanus_genetics/

(This site requires Internet Explorer Version 4 or above).

\section{The Races}

\section{Race Humbloti}

Race humbloti is restricted to the island of Grande Comore in the volcanic Comoro Island group. It is monomorphic, the females being tailed and resembling the males. Both sexes have quite heavy black markings, with a single, very thick black border to the hindwings and pure black tails. The females have much larger black 'epaulette' markings on the forewings than the males.

The butterflies live on the lower slopes of the volcanoes on Grande Comore, and are becoming increasingly scarce as the natural forest is turned to agriculture. This makes individuals particularly hard to get hold of, and it has also proved very hard to breed in this country. 
There is much debate as to whether this race represents the primitive condition for the species (Trimen, 1869 and subsequently Poulton, 1924; Ford, 1936; Clarke \& Sheppard, 1963; Turner, 1963; O'Donald \& Barrett, 1973; Clarke et al., 1985), or whether the monomorphic state is derived (van Bemmelen, 1922; Bernardi, 1963; Vane-Wright \& Smith, 1991). It is interesting that two of the butterflies which act as models for the species on the mainland, Danaus chrysippus and Amauris niavius, are plentiful on the island (pers. obs.). However, it may be that the adults are not under enough predation pressure to lead to mimicry, as the bird fauna is rather restricted and their main predators are likely to be other insects, and lizards.

\section{Race Meriones}

The race meriones is found on the island of Madagascar. It is rather similar to race humbloti, with monomorphic females resembling the males. The black markings are not as heavy as in humbloti, however, especially on the hindwings, where they differentiate into the thicker inner markings and the thin outer black line seen in the mainland races. The tails are also orange towards the tip as on the mainland. The females can be distinguished from the males by the presence of black 'epaulettes' on the forewings.

Individuals from this race are much easier to obtain from breeders than those from humbloti, and are therefore often used in inter-race crosses in order to determine whether or not modifiers are present in the monomorphic races associated with a mimetic ancestor for the race.

\section{Race Dardanus}

This is the most widespread race, its range extending from Sierra Leone to Angola in West Africa, and then east to Uganda and Lake Victoria in Tanzania, where it merges with race meseres.

The females are almost exclusively form hippocoon in the western part of its range, which closely resembles the form hippocoonides in the other races, mimicking Amauris niavius niavius. In the eastern part of its range, the females show increasing proportions of other morphs. In order of decreasing gene dominance, these are:

trophonissa (a variation on trophonius, which forms 'synthetic niobe' or form capenteri when heterozygote with form planemoides)

niobe (occasionally forms 'red-brown cenea' heterozygote with form cenea)

cenea

planemoides (which forms swynnertoni when heterozygote with form cenea) hippocoon 


\section{Race Cenea}

This race is found in South Africa northwards to Delagoa Bay, and merges into race dardanus to the north east and race tibullus to the north west.

The females show six morphs. In order of descending gene dominance, the morphs are:

$\begin{array}{lll}\text { trophonius } & (4 \%) & \text { (can form heterozygote with leighi, salaami which is very rare) } \\ \text { leighi } & (1 \%) & \\ \text { cenea } & (85 \%) & \text { (sometimes co-dominant with leighi) } \\ \text { natalica } & \text { (very rare) } & \text { (can form an unnamed hetrozygote with trophonius) } \\ \text { hippocoonides } & (10 \%) & \end{array}$

\section{Race Tibullus}

This race is found on the east African coast, from Delagoa Bay until somewhere around Mombasa, and on the island of Pemba off the coast of Tanzania. It merges with the form meseres to the west, form cenea to the south, and form polytrophus to the north.

The race is not documented as well as the others, but forms that are known to exist in it include:

$\begin{array}{ll}\text { trophonius } & \text { (uncommon) } \\ \text { prototrophonius } & \text { (uncommon) } \\ \text { salaami } & \text { (extremely rare) } \\ \text { leighi } & \text { (very rare) } \\ \text { trimeni } & \text { (rarely recorded from the mainland) } \\ \text { cenea } & \text { (uncommon) } \\ \text { natalica } & \text { (very rare) } \\ \text { hippocoonides } & \text { (very common) }\end{array}$

On the island of Pemba Cook et al. (1994) report that the forms cenea, salaami, leighi and natalica are not present, and it may be that these forms are only present where the race tibullus meets the adjoining races in which they are present.

\section{Race Meseres}

This race, found in Uganda, and Tanzania east of Lake Victoria, is also known as 'the transitional race', as it forms an intermediate between the race polytrophus to the north and east, race dardanus to the west, and race tibullus to the south (and a little east).

The females show six morphs, some of which are imperfect mimetic forms. Where this is the case, the prefix 'proto' has been used. In order of descending gene dominance, the morphs are: 
prototrophonius

(=lamborni)

planemoides

protosalaami

leighi

protocenea

hippocoonides
$(5 \%)$

(7\%) (also formed as a hetrozygote between planemoides and prototrophonius) (very rarely)

$(60 \%)$

\section{Race Polytrophus}

The race polytrophus is found in the mountains of Tanzania and Kenya east of Lake Victoria, meeting race meseres on the south west and race tibullus on the south east, and race antinorii to the north. It is an unusual race, as the mimicry of the females is often imperfect. This could be because its montane habitat in this region separates it from the model species.

The females show seven morphs. In descending order of genetic dominance, these are:

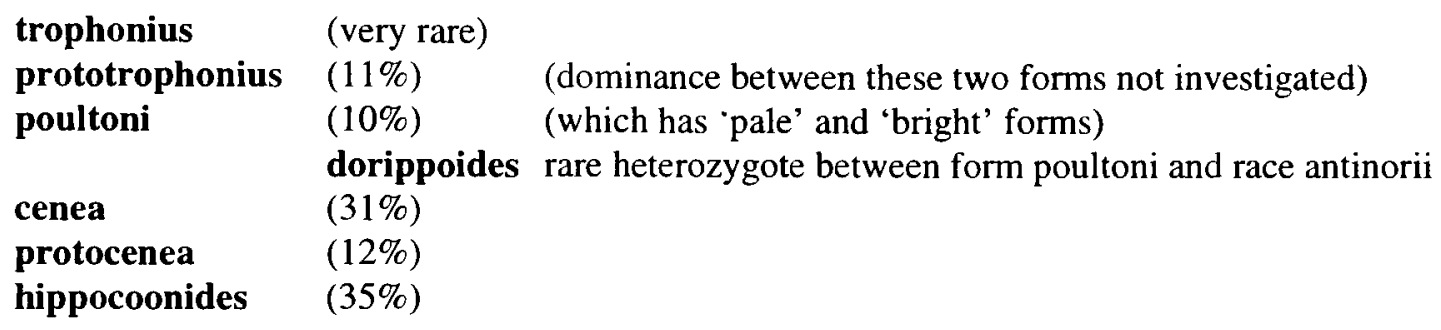

\section{Race Ochracea}

The race ochracea is found on the top of Mount Marsabit in northern Kenya. The females show two morphs only:

$\begin{array}{ll}\begin{array}{l}\text { ochracea } \\ \text { hippocoonides }\end{array} & \text { (dominant, and about 80\%) } \\ \text { (about 20\%) }\end{array}$

\section{Race Flavicornis}

Race flavicornis inhabits the top of Mount Kulal, also in Northern Kenya. It resembles race ochracea except that the tips of the antennae of both sexes are a striking red. The female forms are as in race ochracea.

\section{Race Antinorii}

This race inhabits the region north of Kenya, formerly Abyssinia, meeting race polytrophus to the south. It is an unusual race in that the females all possess tails like the males even though some of them are mimics of tailless species. The race is difficult to obtain, and has only been studied a little. 
The females are all tailed, but show several different morphs:

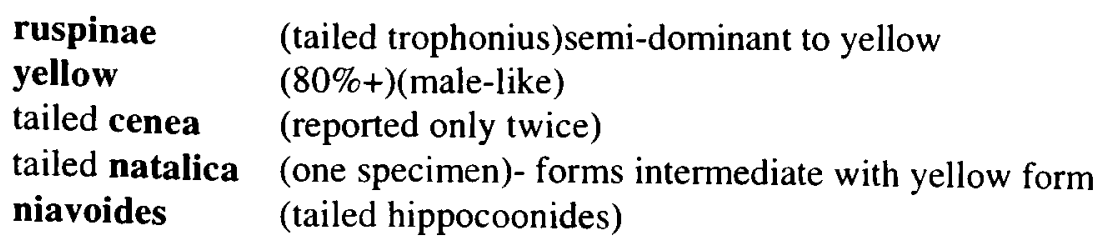

The tails are controlled by a single major gene with modifiers, tailless ( $\mathrm{T}$ ) being semidominant to tailed $(t)$. The locus is independent of wing pattern.

\section{The morphs}

Males

The males of Papilio dardanus are similar throughout the range of the species, although the extent of the black markings varies. In race humbloti, the black border to the hindwings is solid and the tails are also completely black. The females in races humbloti and meriones, and the yellow form in race antinorii resemble the males (gene $\mathrm{H}^{\mathrm{y}}$ ).

\section{Trimeni}

This morph resembles a tail-less male and is found in race tibullus on the east African coast.

\section{Hippocoonides /Hippocoon}

This widespread black and white morph mimics Amauris niavius. Hippocoon (which has more obvious rays on the hindwings than hippocoonides) is found in race dardanus in West Africa, and mimics Amauris niavius niavius, and the very similar hippocoonides mimics Amauris niavius dominicanus, and is found in races cenea, tibullus, meseres, polytrophus, ochracea, and flavicornis (where it has red tips to the antennae). It is genetically the bottom recessive (gene h).

\section{Niavoides}

This is the tailed form of hippocoonides, found in the northern race antinorii, where it roughly mimics Amauris niavius.

\section{Natalica}

This is a non-mimetic form which resembles hippocoonides except that the white areas are all buff. It is found in race cenea, and a tailed version exists in race antinorii. (gene $\mathrm{H}^{\mathrm{na}}$ ). 


\section{Cenea}

This is a mimic of Amauris echeria echeria and Amauris albimaculata, and is found in races cenea, dardanus, polytrophus, and probably tibullus. A tailed form is found in race antinorii. (gene $\mathrm{H}^{\mathrm{c}}$ ).

\section{Protocenea}

This is an incomplete version of cenea, where fluorescent yellow pigment (Papiliochrome II) is present to some extent. It is found in races polytrophus and meseres.

\section{Ochracea}

This larger form of cenea, with more heavily spotted hindwings and all the whites replaced by buff, is a mimic of Amauris echeria septentrionalis, and is found in races ochracea and flavicornis (where it has red-tipped antennae). (gene $\mathrm{H}^{\circ}$ ).

\section{Trophonius (White lamborni)}

This form mimics Danaus chrysippus, and is found in races cenea, polytrophus and tibullus, and as form trophonissa (with more obvious rays on the hindwings) in race dardanus. It is dominant to other forms (gene $\mathrm{H}^{\mathrm{T}}$ ).

\section{Prototrophonius (Yellow Lamborni)}

This is the incomplete mimetic form, in which the white areas on the forewings are replaced with fluorescent yellow. It is found in races polytrophus, tibullus and meseres.

\section{Ruspinae}

This is the tailed form of trophonius found in race antinorii.

\section{Leighi}

This is a non-mimetic form with light orange markings on the forewing and buff on the hindwing. It occurs in races cenea and meseres. $\left(\right.$ Gene $\mathrm{H}^{\mathrm{L}}$ ).

\section{Planemoides}

This morph rather resembles leighi, but with slightly more orange on the forewings, and the buff hindwing colour replaced with white. It is a mimic of Bematistes poggei, and is found in races dardanus and meseres. (Gene $\mathrm{H}^{\mathrm{Pl}}$ ). 


\section{Swynnertoni}

This is an incompletely developed form of planemoides in which the bases of the hindwings are buff and the black bar on the forewings is just about complete. It is a heterozygote between planemoides and cenea (genotype $\mathrm{H}^{\mathrm{Pl}} \mathrm{H}^{\mathrm{c}}$ ).

\section{Niobe}

This entirely bright orange morph mimics Bermatistes tellus. It occurs in race dardanus. (Gene $\mathrm{H}^{\mathrm{Ni}}$.

\section{Salaami}

This is a non-mimetic form very like niobe, but in which the orange on the forewings is slightly lighter than that on the hindwings. It is found in race cenea as a heterozygote of trophonius and leighi (genotype $\mathrm{H}^{\mathrm{T}} \mathrm{H}^{\mathrm{L}}$ ). A form called protosalaami, in which the black bar across the forewings is less distinct, is found in race polytrophus, and occasionally in meseres.

\section{Dorippoides}

This is a form of salaami in which the black bar across the forewings is completely absent, making the forewings almost entirely orange. It is formed in race polytrophus by interbreeding between race antinorii and form poultoni of race polytrophus.

\section{Poultoni}

This is also very similar to niobe, but the hindwings are slightly less rayed and the body is grey or fawn with black dots rather than orange. It has two forms - pale and bright - although the relationship between these forms in uncertain. It occurs in race polytrophus (genes $\mathrm{H}^{\mathrm{pp}}$ or $\mathrm{H}^{\mathrm{bp}}$ ).

\section{$\underline{\text { References }}$}

Bernardi, G. 1963. Quelques aspects zoogéographiques du mimétisme chez les Lépidpidoptères. Proceedings, $16^{\text {th }}$ International Congress of Zoology, Washington 4, 161-166.

Clarke, C.A. \& Sheppard, P.M. 1959. The genetics of Papilio dardanus, Brown. I race cenea from South Africa. Genetics 44: 1347-1358.

Clarke, C.A. \& Sheppard, P.M. 1960a. The genetics of Papilio dardanus, Brown. II races dardanus, polytrophus, meseres and tibullus. Genetics 45: 439-457.

Clarke, C.A. \& Sheppard, P.M. 1960b. The genetics of Papilio dardanus, Brown. IIl race antinorii from Abyssinia and race meriones from Madagascar. Genetics 45: 683-698.

Clarke, C.A. \& Sheppard, P.M. 1962. The genetics of Papilio dardanus, Brown. IV data on race ochracea, race flavicomis, and further information on races polytrophus and dardanus. Genetics 47 : 909-920. 
Clarke, C.A. \& Sheppard, P.M. 1963. Interactions between major genes and polygenes in the determination of mimetic patterns of Papilio dardanus. Evolution 17, 404-413.

Cook, S.E., Vernon, J.G., Bateson, M., Guilford, T. 1994. Mate choice in the polymorphic African swallowtail butterfly, Papilio dardanus - male-like females may avoid sexual harassment. Animal Behaviour 47 (2), 389-397.

Ford, E.B. 1936. The genetics of Papilio dardanus Brown (Lep.). Transactions of the Royal Entomological Society of London 85, 435-466.

O'Donald, P. \& Barrett, J.A. 1973. Evolution of dominance in polymorphic Batesian mimicry. Theoretical Population Biology 4, 173-192.

Poulton, E.B. 1924. Papilio dardanus. The most interesting butterfly in the world. Journal of the East African and Ugandan Natural History Society 20, 4-22.

Trimen, R. 1869. On some remarkable mimetic analogies among African butterflies. Transactions of the Linnean Society of London 26, 497-522.

Turner, J.R.G. 1963. Geographical variation and evolution in the males of the butterfly Papilio dardanus Brown (Lepidoptera: Papilionidae). Transactions of the Royal EntomologicalSociety of London 115, 239-259.

Van Bemmelen, J.F. 1922. The wing-design of mimetic butterflies. Proceedings, Section of Sciences, Koninklijke Nederlandse Akademie Wetensschappen 23, 877-886.

Vane-Wright, R.I. \& Smith, C.R. 1991. Phylogenetic relationships of three African swallowtail butterflies, Papilio dardanus, P. phorcas, and P. constantinus: a cladistic analysis (Lepidoptera: Papilionidae). Systematic Entomology 16: 275-291. 


\section{Appendix 2}

\section{Electroretinogram calibration}

\section{$\underline{\text { Introduction }}$}

This appendix concerns the calibration of the initial response spectrum measured for butterflies using an electroretinogram (Experiment 2-4 and Experiment 3-5) to take into consideration the intensity of the mercury lamp at different wavelengths, and to create a 'response surface' with varying wavelengths and intensities.

\section{$\underline{\text { Method \& Materials }}$}

Firstly the intensity spectrum of the lamp was measured using a photodiode, RS stock number 305-462 (RS Electronics ${ }^{*}$ ) in the light-level sensor circuit (see Figure A2-1). The lamp beam was passed through the monochromator, exactly as in the ERG experiment, and the photodiode was placed in the focused, monochromated beam. The response to flashes of light of different wavelengths from the photodiode circuit was recorded on the Lloyd Instruments PL3 chart recorder.

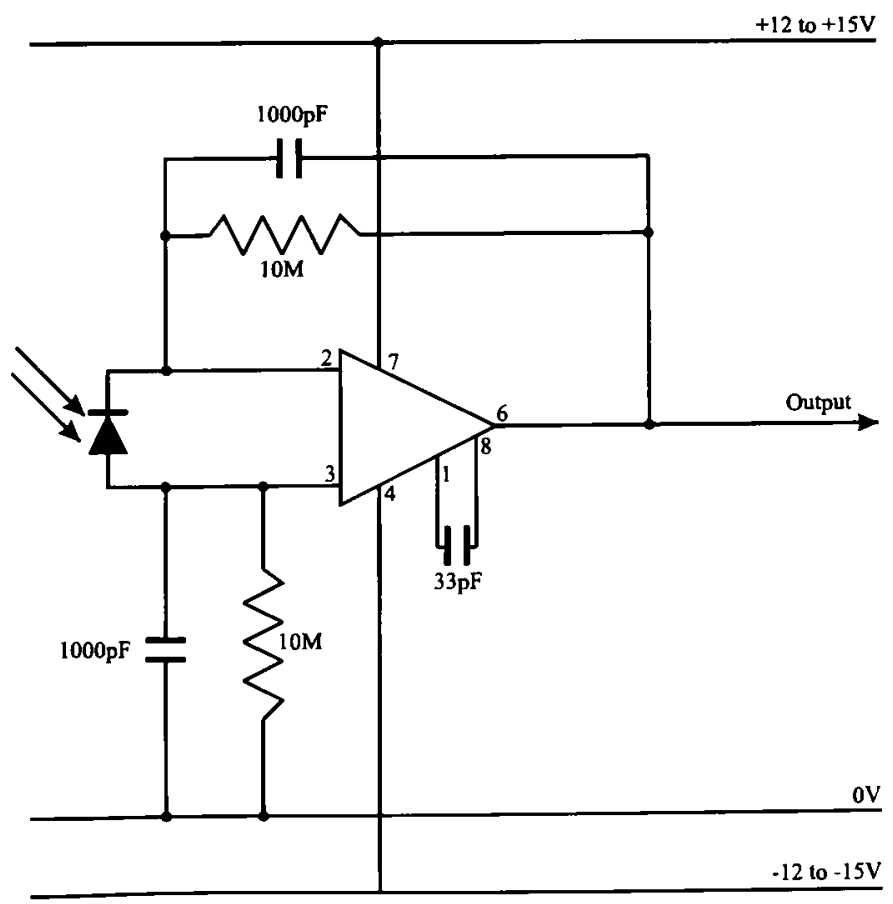

Figure A2-1 Photodiode circuit used for measurement of lamp intensity at different wavelengths.

The height of the resulting peaks was measured and normalised, and plotted as a graph of photodiode response against wavelength (see Figure A2-2). 


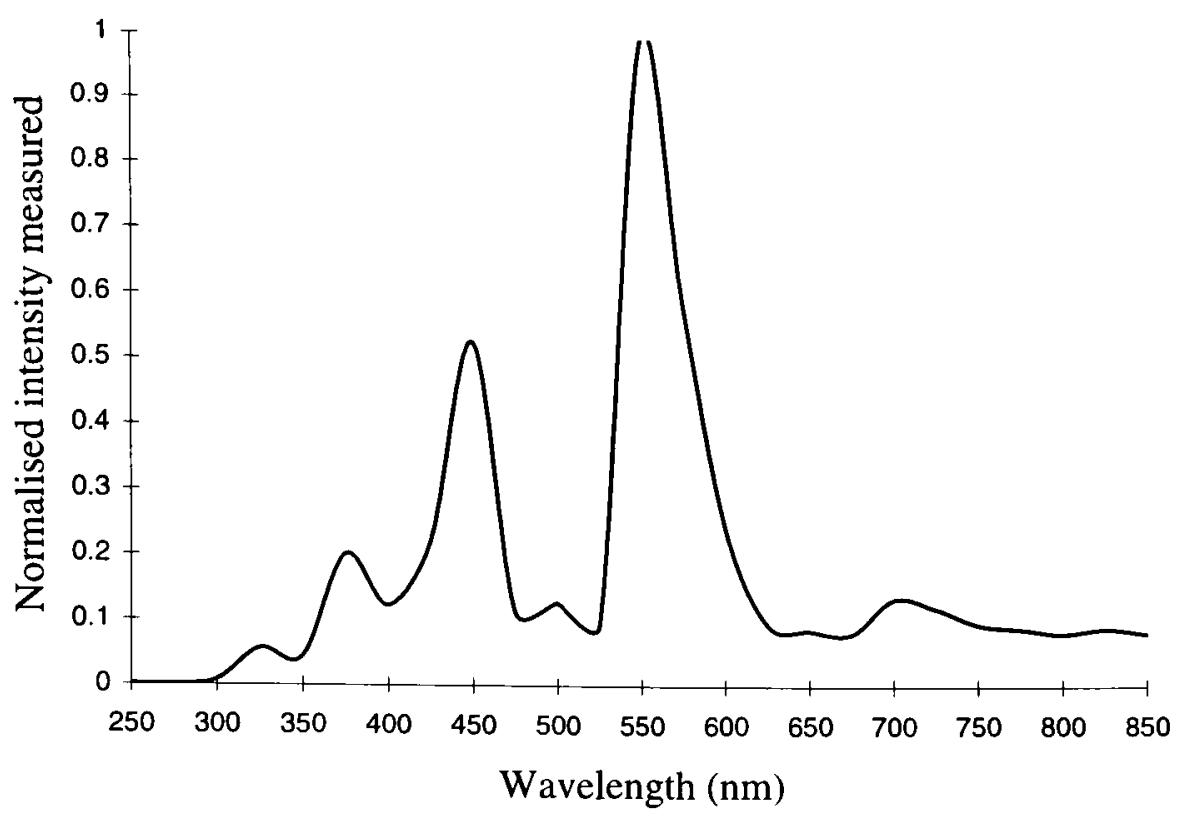

Figure A2-2 Graph of response of photodiode against wavelength for the mercury lamp.

This graph then had to be adjusted for the wavelength-dependent sensitivity of the photodiode itself, as given by the manufacturer, and normalised to give the true lamp power at different wavelengths (see Figure A2-3).

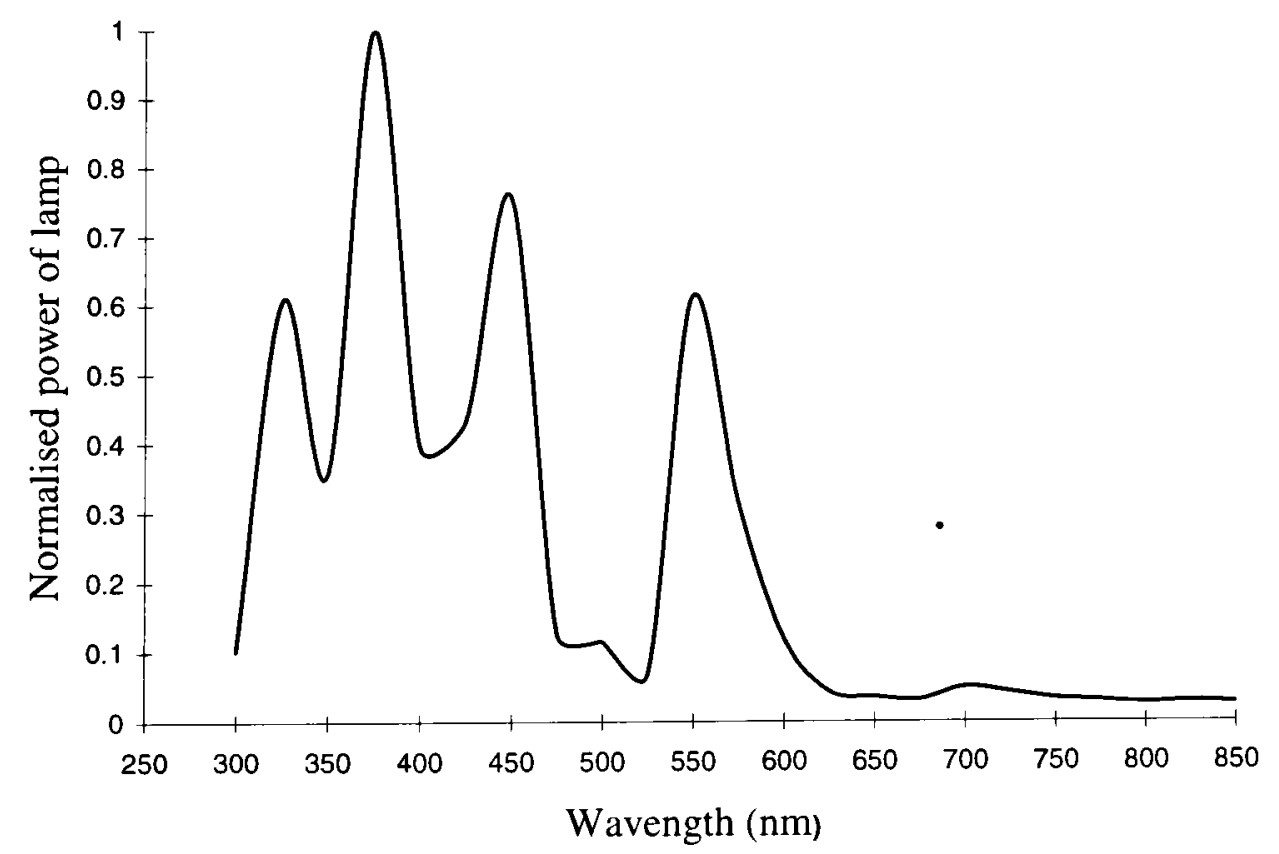

Figure A2-3 Graph of the relative intensity of the mercury lamp at the different wavelengths used.

The photodiode measured the power of the lamp at these wavelengths, but the actual quantum flux is what affects the visual pigments of the butterfly. The energy of the photons and hence the power is a function of the wavelength of the light, and a relative quantum flux can be obtained at a given wavelength simply by multiplying the relative power by the wavelength (in $\mathrm{nm}$ ), and then 
renormalising. This produces a final graph of the relative quantum flux at each wavelength (see Figure A2-4)

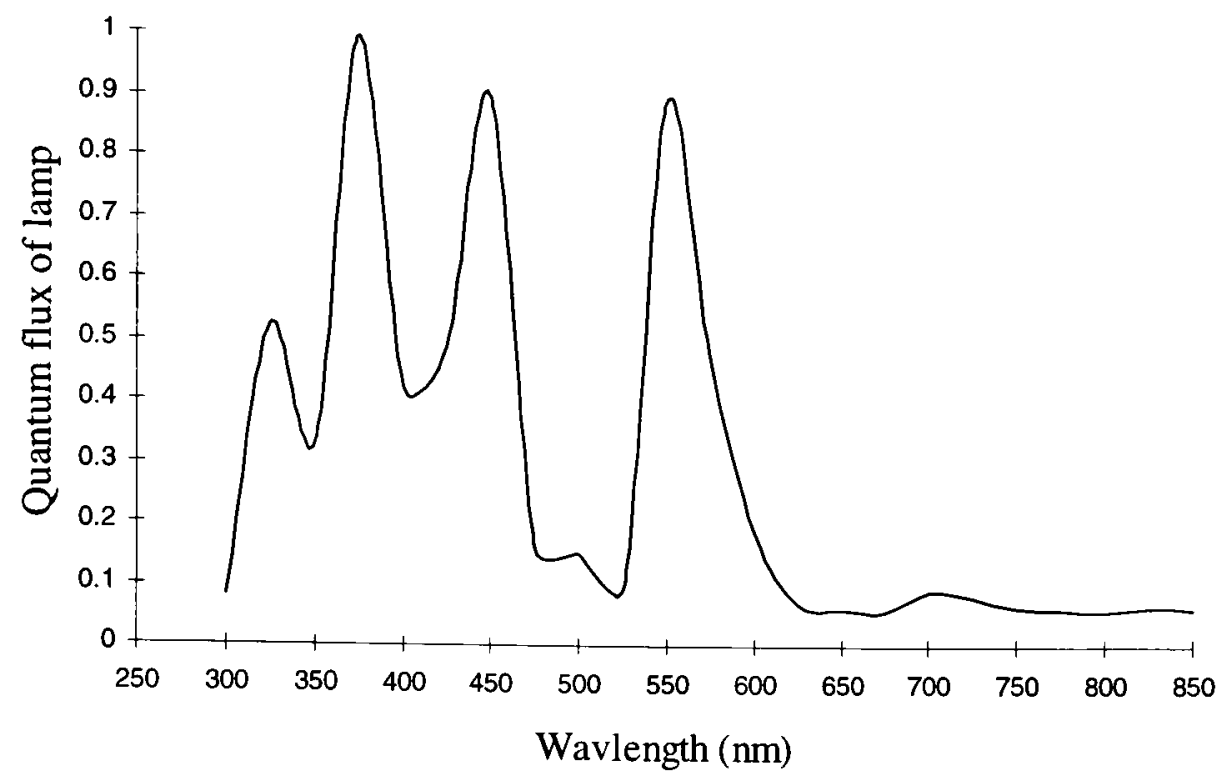

Figure A2-4 Graph of the relative quantum flux at each wavelength.

Having determined the intensity of the lamp at the different wavelengths, it was necessary to measure the intensity-response function of the butterfly at the wavelengths where the higher lamp intensity needed to be accounted for. The wavelengths which had the highest intensity were $375 \mathrm{~nm}$, $450 \mathrm{~nm}$ and $550 \mathrm{~nm}$. These were close to the sensitivity peaks of the three main photoreception pigments found in insects (Chittka \& Menzel, 1992, Backhaus \& Menzel, 1992, Bennett et al., 1997, Bernard \& Remington, 1991, Arikawa et al., 1987), at approximately $360 \mathrm{~nm}, 460 \mathrm{~nm}$, and $520 \mathrm{~nm}$. Therefore measurements of the intensity-response function at the $375 \mathrm{~nm}, 450 \mathrm{~nm}$, and $550 \mathrm{~nm}$ done on several individuals were used to assess the similarity of the intensity function from different individuals.

A series of filters (Supergel swatch set, Rosco Filters*; Lee numerical swatch set, Lee Filters $^{* *}$ ) were used to achieve a range of intensities at these three wavelengths. The intensity of the light once passed through the filter was measured using the photodiode circuit, and a suitable series of filters selected for each wavelength to give a good spread of intensities (see Table A2-1 at end of Appendix for list of filters used).

The response of between three and five individual butterflies was then recorded as an ERG (for method, see Experiments 2-4 and 3-5). Unfortunately, only Papilio dardanus specimens could be obtained. However, the similarity of the response curves of Argynnis paphia and Papilio dardanus (shown in the results sections of Experiment 2-4 and 3-5 respectively) indicate that they may indeed 
have similar intensity-response functions, and therefore that using the intensity-response curves of Papilio dardanus to calibrate the spectral efficiency curve of Argynnis paphia may be a reasonable approximation.

a) Mean intensity response function at $550 \mathrm{~nm}$

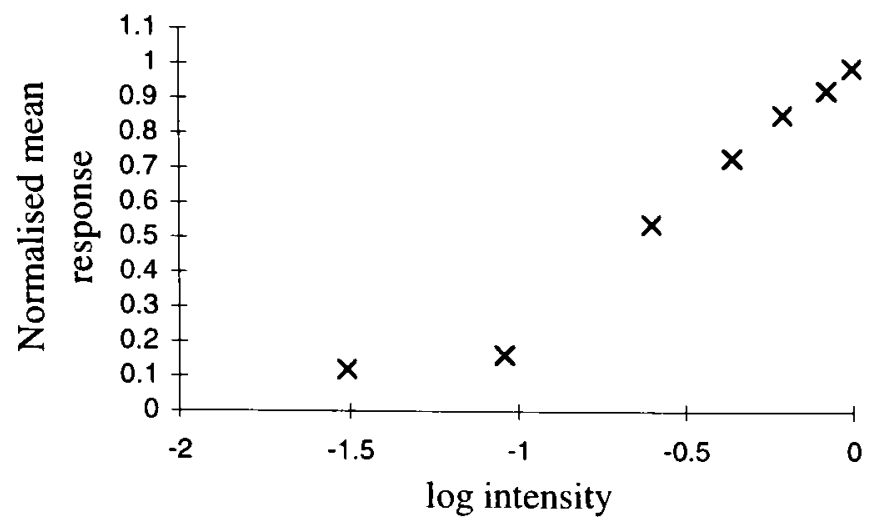

b) Mean intensity response function at $450 \mathrm{~nm}$

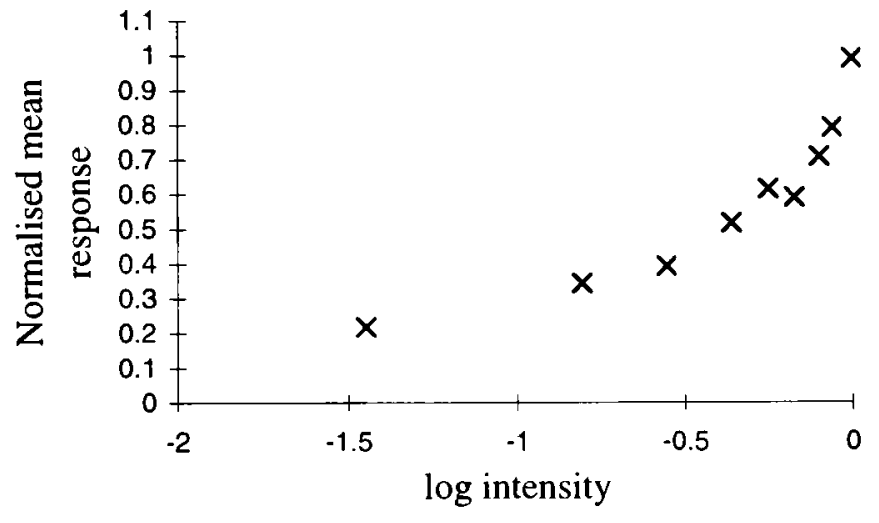

c) Mean intensity response function at $375 \mathrm{~nm}$

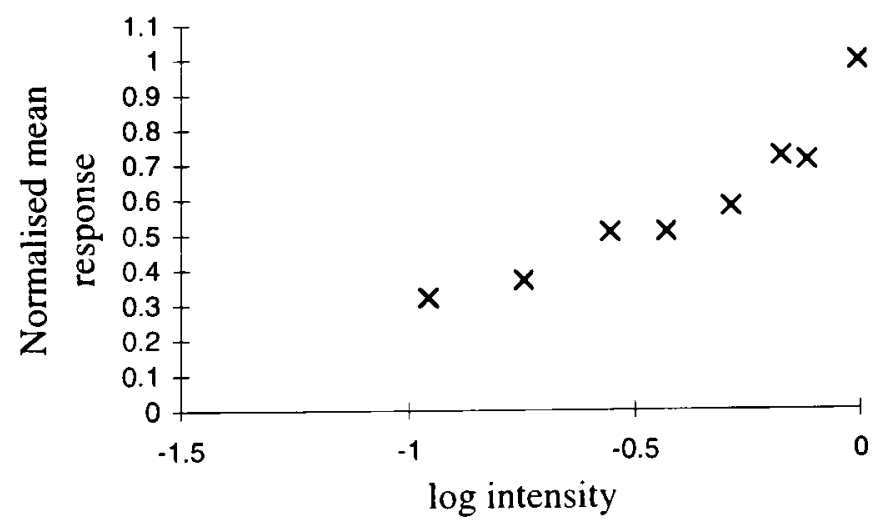

Figure A2-5 The mean intensity-response functions of 3 to 5 individual Papilio dardanus at a) $550 \mathrm{~nm}$, b) $450 \mathrm{~nm}$, and c) $375 \mathrm{~nm}$.

This data indicated that the intensity response functions were smooth curves (plotted on a log scale), and that data of sufficient quality could be obtained to predict the curve with reasonable accuracy from the data of one individual. This was important, as different individuals are inevitably illuminated to different degrees due to their placement in the light beam, and thus each would give slightly shifted intensity functions. Therefore data from several individuals cannot be combined.

Therefore one individual was used, and about 6 measurements of the response were taken for both the full and filtered intensity in each case. The graphs show the ratio of the mean value of the response to the filtered intensity to the mean value of the response to the unfiltered intensity as measured immediately prior to it.

The functions obtained from a single individual were mostly relatively smooth curves (see Figure A2-6), and the function:

$$
y=\frac{a x}{(1-b x)+1}
$$


was fitted to each (where $a$ and $b$ are variable parameters controlling the scale and curvature of the function respectively).

a) Intensity response at $325 \mathrm{~nm}$

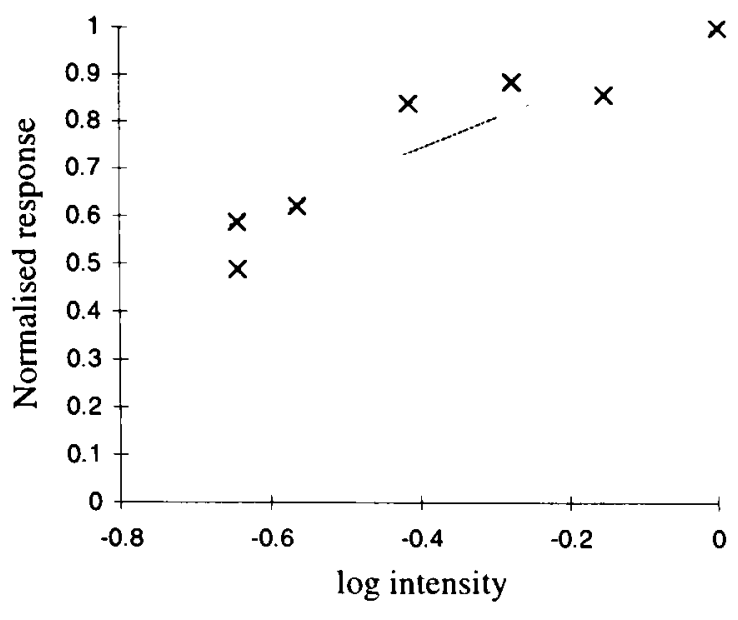

c) Intensity response at $375 \mathrm{~nm}$

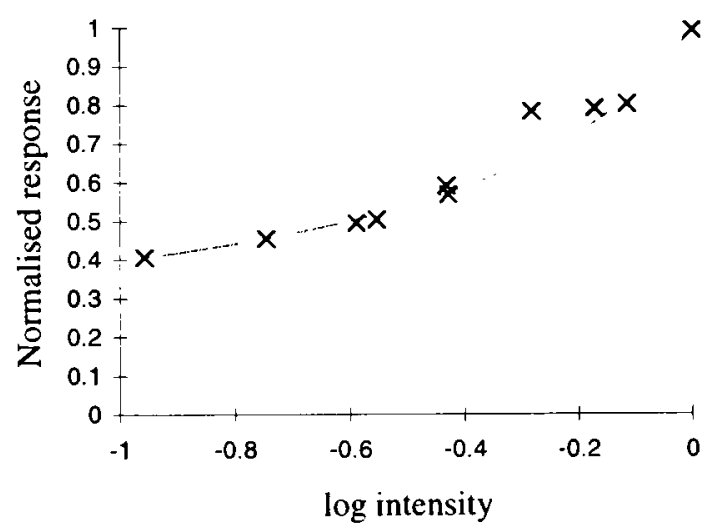

e) Intensity response at $425 \mathrm{~nm}$

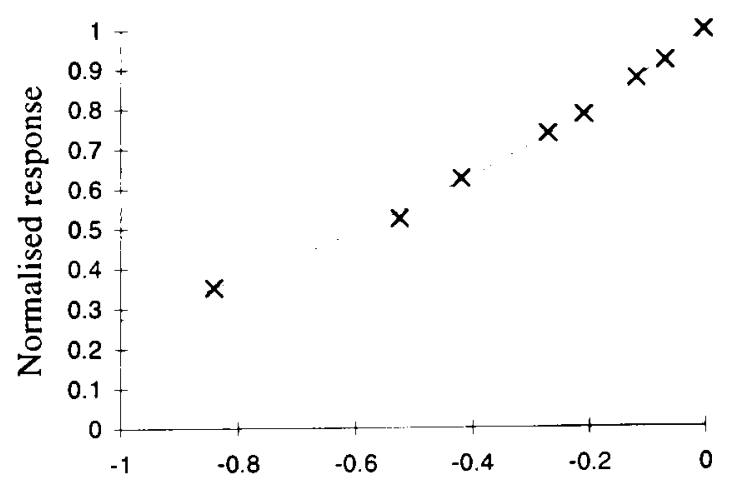

b) Intensity response at $350 \mathrm{~nm}$

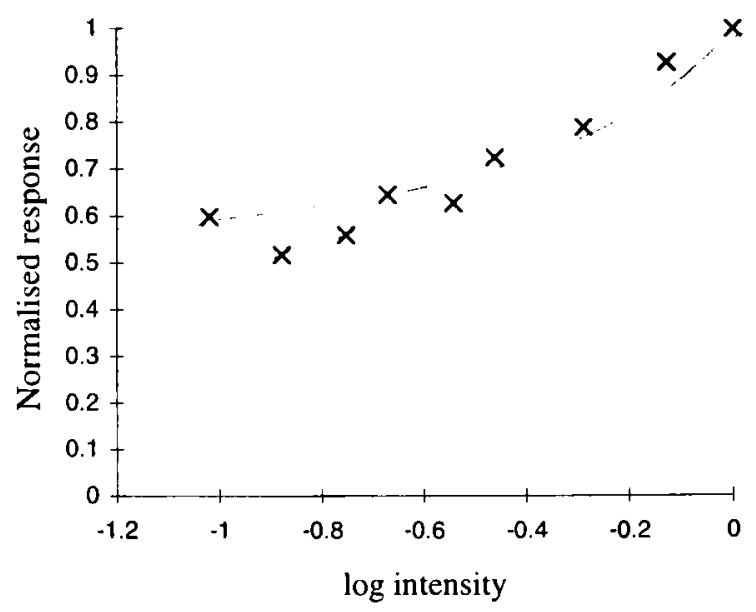

d) Intensity response at $400 \mathrm{~nm}$

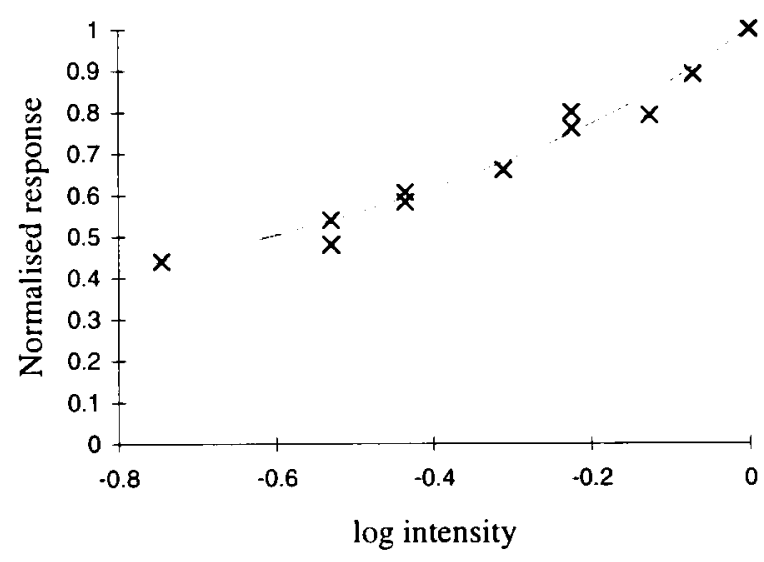

f) Intensity response at $450 \mathrm{~nm}$

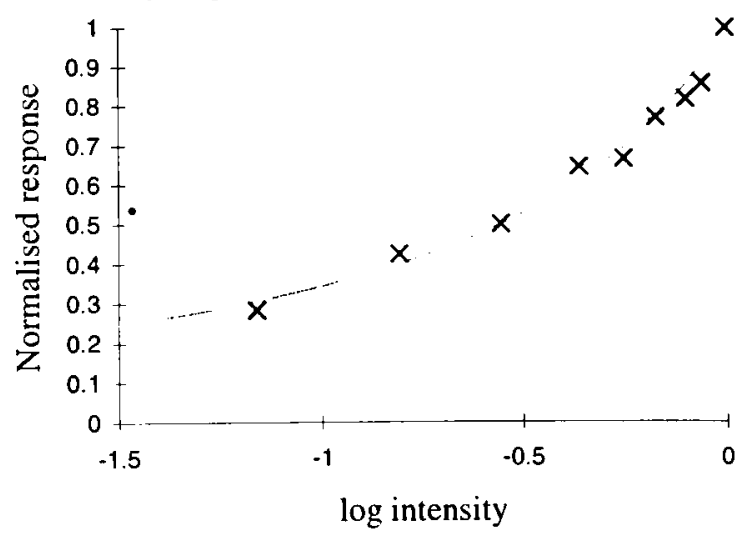


Appendix 2 Calibration of the electroretinogram data

g) Intensity response at $475 \mathrm{~nm}$

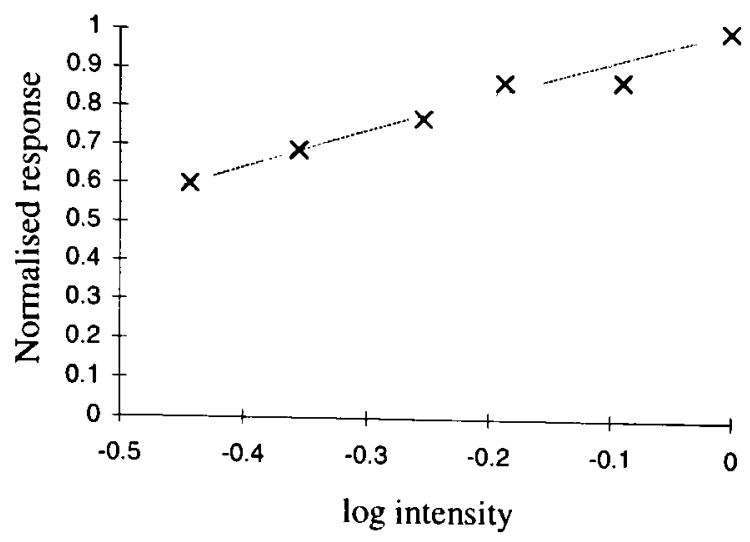

h) Intensity response at $500 \mathrm{~nm}$

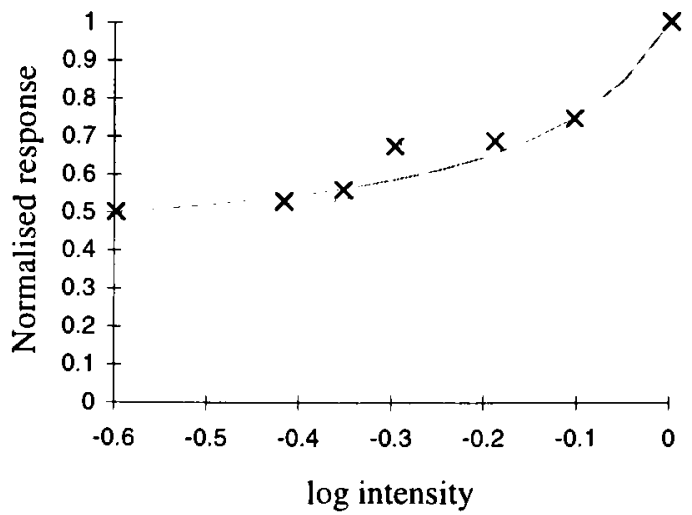

i) Intensity response at $525 \mathrm{~nm}$

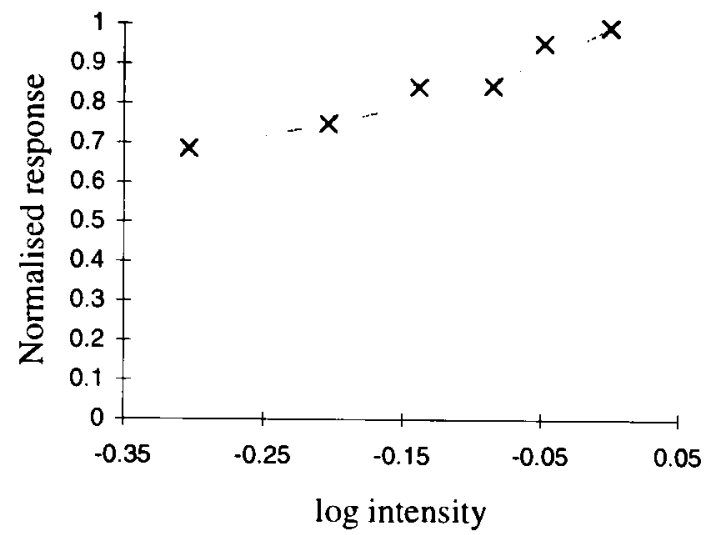

k) Intensity response at $575 \mathrm{~nm}$

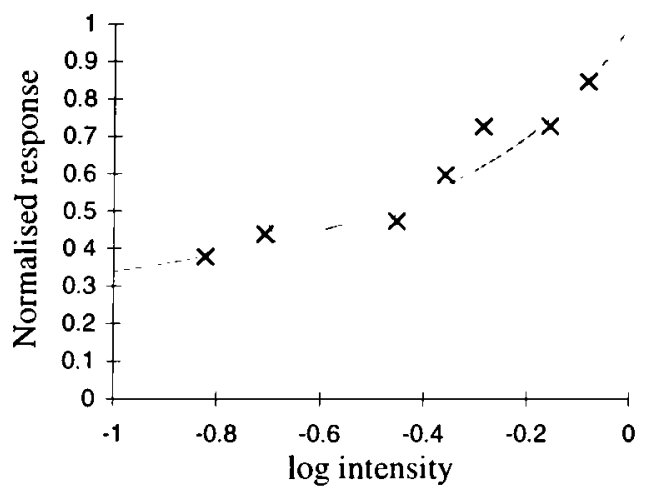

j) Intensity response at $550 \mathrm{~nm}$

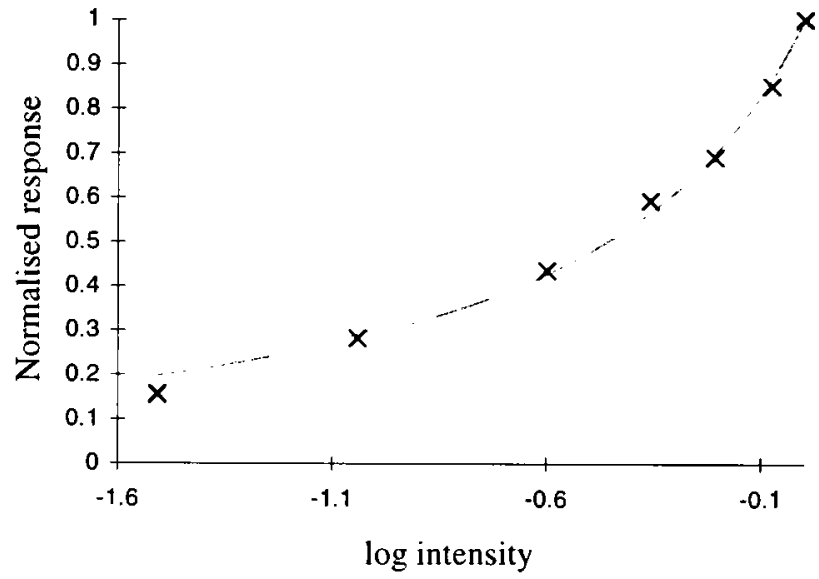

1) Intensity response at $600 \mathrm{~nm}$

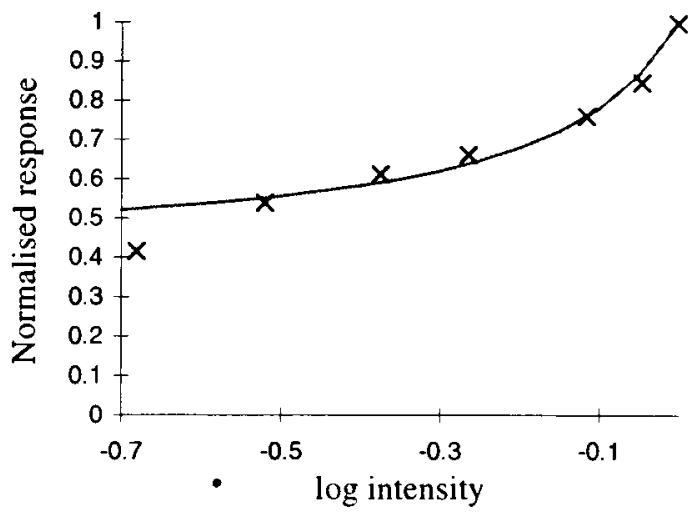

Figure A2-6 The relative intensity functions for Papilio dardanus at a) $325 \mathrm{~nm}$, b) $350 \mathrm{~nm}$, c) $375 \mathrm{~nm}$, d) $400 \mathrm{~nm}$, e) $425 \mathrm{~nm}$, f) $450 \mathrm{~nm}$, g) $475 \mathrm{~nm}$, h) $500 \mathrm{~nm}$, i) $525 \mathrm{~nm}$, j) $550 \mathrm{~nm}$, k) $575 \mathrm{~nm}$, l) $600 \mathrm{~nm}$.

Therefore, a corrected response figure was calculated for each wavelength for which the intensity was higher than the minimum for both species, giving corrected spectral efficiency functions (shown in Figure A2-7). 
a)

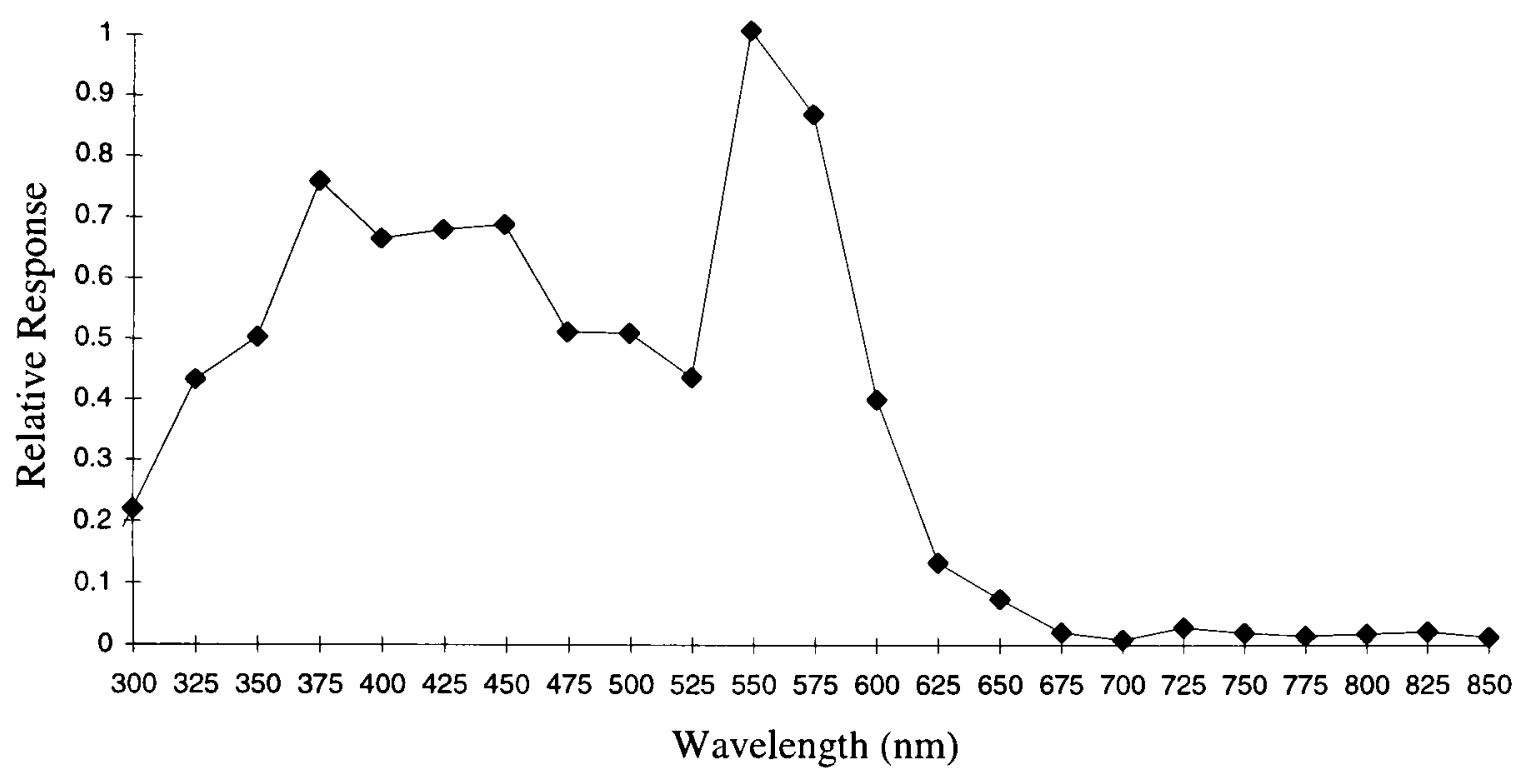

b)

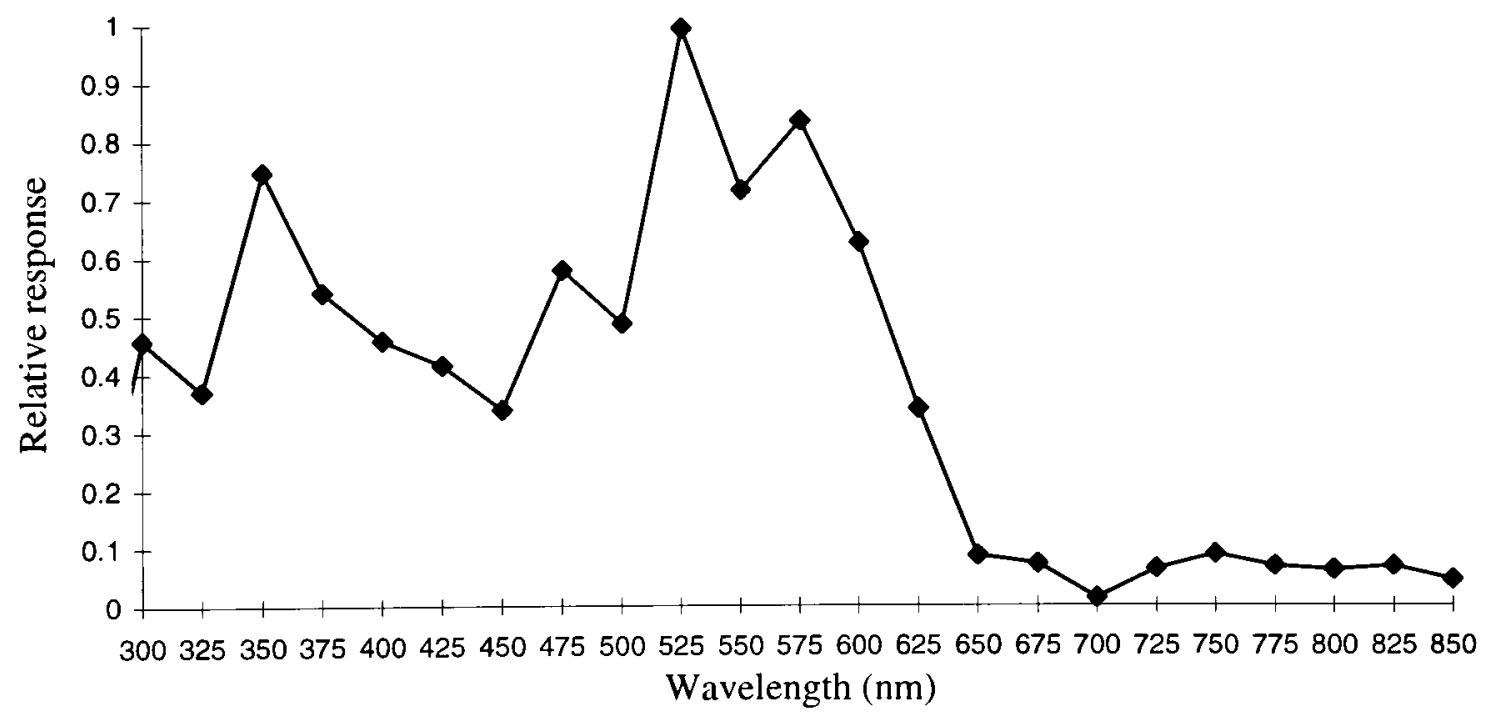

Figure A2-7 The corrected spectral efficiency functions of a) Papilio dardanus and b) Argynnis paphia (using the intensityresponse functions of Papilio dardanus).

Figure A2-6, however, demonstrates how the spectrabefficiency function may vary depending on the intensity at which it is measured. Therefore a more useful representation of the visual spectrum of a butterfly would be represented as a surface, taking into account both wavelength and intensity. Surfaces were therefore plotted for Papilio dardanus representing both the raw data, and a surface composed of the smoothed intensity functions shown in Figure A2-6. These surfaces are shown in the results of Experiment 3-5. 


\begin{tabular}{|c|c|c|c|c|c|c|c|c|c|c|c|c|}
\hline \multirow{2}{*}{$\begin{array}{c}\text { Filter } \\
\text { Number }\end{array}$} & \multicolumn{12}{|c|}{ Relative log intensity at wavelength ( $\mathrm{nm}$ ) (uncalibrated for photodiode sensitivity): } \\
\hline & 325 & $350^{\circ}$ & 375 & 400 & 425 & 450 & 475 & $\mathbf{5 0 0}$ & 525 & 550 & 575 & 600 \\
\hline Rosco 00 & -0.153 & -0.129 & & & & & & & & & & \\
\hline Rosco 052 & -0.643 & & & & & & & & & & & \\
\hline Rosco 053 & -0.404 & & & & & & & & & & & \\
\hline Rosco 054 & -0.414 & & & & & & & & & & & \\
\hline Rosco 372 & -0.425 & & & & & & & & & & & \\
\hline Rosco 373 & -0.276 & & & & & & & & & & & \\
\hline Lee 002 & & -1.878 & -0.365 & & & -0.172 & -0.253 & -0.416 & & & -0.823 & -0.368 \\
\hline Lee 003 & & -0.378 & & & & & -0.089 & -1.04 & -0.139 & & & \\
\hline Lee 004 & & -0.833 & & & & & & & & & & \\
\hline Lee 007 & & & & & & & -0.186 & & -0.085 & -0.059 & -0.051 & \\
\hline Lee 008 & & & & & -0.841 & -1.021 & & & & & & \\
\hline Lee 009 & & -0.753 & -0.429 & -0.435 & -0.419 & -0.428 & & \begin{tabular}{l|l}
-0.297 \\
\end{tabular} & & & & -0.073 \\
\hline Lee 013 & & -1.020 & -0.554 & -0.530 & -0.524 & -0.553 & -0.537 & & & -0.170 & & \\
\hline Lee 015 & & & & & & & & & & -0.206 & -0.081 & -0.074 \\
\hline Lee 017 & & & & & & -1.447 & & & & & -0.708 & \\
\hline Lee 021 & & & & & & & & & & & -0.380 & \\
\hline Lee 022 & & & & & & & & & & & & -0.352 \\
\hline Lee 024 & & & & & & -1.160 & & & & & 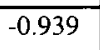 & \\
\hline Lee 025 & & & & & & & & & & -1.040 & & \\
\hline Lee 035 & & & & & & & & -0.271 & & & & \\
\hline Lee 036 & & & & & & & & & & -0.556 & -0.411 & \\
\hline Lee 039 & -0.644 & -0.288 & & & & & & -0.189 & & & -0.286 & \\
\hline Lee 048 & & & & & & -0.361 & -0.443 & & & -1.507 & & \\
\hline Lee 052 & & & & & & -0.136 & & & & & & -0.520 \\
\hline Lee 053 & & -0.370 & -0.172 & -0.126 & -0.118 & -0.098 & & & & & -0.276 & \\
\hline Lee 055 & & & & & & & & & & & & -0.258 \\
\hline Lee 058 & & & -0.957 & & & -0.249 & -0.355 & & & & & \\
\hline Lee 061 & & & -0.480 & & & -0.059 & & & & & -0.245 & \\
\hline Lee 063 & & & & & & -0.065 & -0.077 & & & & & -0.331 \\
\hline Lee 068 & & & & & & & & -0.352 & & & & \\
\hline Lee 071 & & & & & & -0.699 & & & & & & \\
\hline Lee 075 & & & & & & & \begin{tabular}{|c|}
-0.219 \\
\end{tabular} & & & & & \\
\hline Lee 079 & & & & -0.745 & & & & & & & & \\
\hline Lee 088 & & & & & & & & & & & -0.155 & \\
\hline Lee 089 & & & & & & & & & & -0.357 & -0.666 & \\
\hline Lee 100 & & -1.092 & & & & & $\cdot$ & & & & & \\
\hline Lee 102 & & & -0.528 & & & & & & & & & \\
\hline Lee 104 & & & & & & & & & & & & -0.046 \\
\hline Lee 105 & & & & & & & & & & & -0.147 & \\
\hline Lee 107 & & & -0.576 & & & -0.286 & & -0.598 & & & & \\
\hline Lee 109 & & & -0.746 & -0.311 & -0.271 & -0.286 & & & & & & -0.115 \\
\hline Lee 111 & & & & & & & & & & & & -0.264 \\
\hline Lee 116 & & & & & & -1.047 & & & & & & \\
\hline Lee 130 & -0.563 & -0.214 & -0.115 & -0.071 & -0.068 & & -0.065 & & -0.048 & & & \\
\hline Lee 131 & & & & & & & & & -0.204 & & & \\
\hline Lee 134 & & & & & & & & & & -0.598 & -0.359 & -0.242 \\
\hline Lee 135 & & & & & & & & & & & -0.696 & \\
\hline Lee 136 & & -0.671 & -0.365 & & & & & & & & & \\
\hline Lee 137 & & & & & & & & & & & & -0.681 \\
\hline Lee 151 & & & & & & & & & & -0.273 & & \\
\hline
\end{tabular}


Appendix 2 Calibration of the electroretinogram data

\begin{tabular}{|l|l|l|l|l|l|l|l|l|l|l|l|l|}
\hline Lee 153 & & & -0.432 & & & & & & & & & \\
\hline Lee 154 & & -0.542 & -0.284 & -0.224 & -0.209 & & & & & & & \\
\hline Lee 162 & & & -0.256 & & & & & & & & & \\
\hline Lee 169 & & & -0.256 & & & & & & & & & \\
\hline Lee 170 & & & & & -0.301 & & & & & & \\
\hline Lee 198 & & & & & & -0.805 & & & & & & \\
\hline Lee 209 & & & -0.589 & & & & & & & & & \\
\hline Lee 241 & & & & & & & & & & & -0.625 & \\
\hline Lee 242 & & & & & & & & & & -0.453 & \\
\hline Lee 244 & & & & & & & & & & & -0.118 & \\
\hline Lee 246 & & -0.461 & -0.245 & & & & & & -0.082 & -0.076 & & -0.095 \\
\hline Lee 279 & & & -0.126 & & & & & & & & & \\
\hline
\end{tabular}

Table A2-1 The measured intensity of the filtered light using different filters at each wavelength (not adjusted for the sensitivity curve of the photodiode itself) 


\section{Appendix Three}

\section{The flight cage used in behavioural experiments}

All behavioural experiments with Papilio dardanus were carried out in a flight cage in a heated greenhouse, kept at between $30-40^{\circ}$ Centigrade. A diagram of the cage is shown in Figure A31. The high temperature and the introduction of plants were necessary for the butterflies to fly around the cage and to encounter each other. The plants included a Citrus, on which females were allowed to lay. The $1^{\text {st }}$ instar larvae were removed, and reared on Choisya ternata. The flowers in the centre of the cage were either used in Experiments (in Chapter 4), or when flower choice experiments were not being carried out, they were kept filled with sugar water.

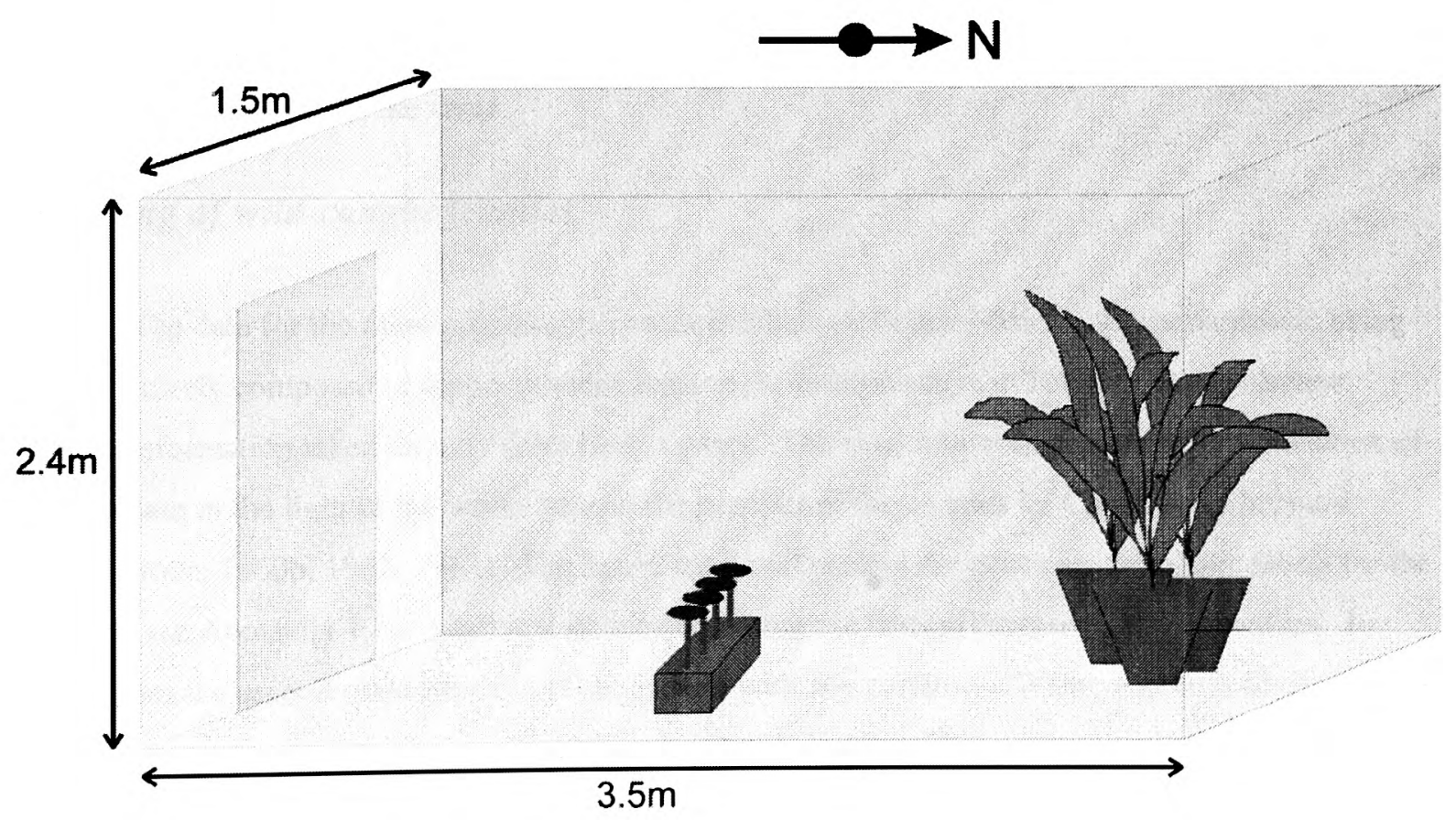

Figure A3-1 The flight cage used in behavioural experiments with Papilio dardanus

The cage was made of very fine nylon netting, with the walls black and the roof white. A door was made at the South end of the cage, kept sealed with Velcro, to allow entry and exit. The cage had no floor, but was weighted to the concrete flooring of the greenhouse. 


\section{Appendix Four}

\section{Analysis of data from Ford (1936)}

\section{The data}

Ford collated records of female offspring of wild-caught females from various sources (see Ford. 1936 for references), together with approximate percentages of the morphs found in the populations from which the females came.

These appear to be the only records of the offspring from wild-caught females, who had mated in the wild, and therefore represent the only way in which the degree of assortative mating and multiple mating occurring in the wild may be assessed without making further collections.

Throughout this appendix the prefix 'proto-' usually given to imperfectly mimetic forms is dropped, and the categories of 'imperfect' and 'perfect' are merged, as the major mimetic genes carried by both forms are the same.

\section{Offspring of wild-caught females}

The data for the more polymorphic races is tabulated below (data from race dardanus, being almost entirely composed of hippocoonides females, is uninformative). The first three columns contain information taken directly from Ford's paper. The final four columns are an interpretation of the raw data in the light of the work done on the genetics of the morphs by Clarke and Sheppard $(1959,1960 \mathrm{a}, 1960 \mathrm{~b}, 1962,1963)$. The letters used to represent the genes are the allelic labels for the locus $\mathrm{H}$ (see Appendix 1 for details), with $\mathrm{h}$ representing the bottom recessive, hippocoonides. In many cases the second male gene cannot be known with any certainty. Where a gene is likely, it is labelled with a '?', and where one alternative is possible but less likely, it is put in brackets. Where more than two genes are possible, the cell is left blank. The minimum number of matings possible to achieve the observed brood has been assumed in all cases, but where a second mating must have occurred to produce the number of morphs seen, a second line has been inserted for the second male. In these cases, the second gene of one male is often known, but not which male carried it, so it again has a '?' after it. 


\begin{tabular}{|c|c|c|c|c|c|c|}
\hline Race & Morph & Offspring & female gene 1 & female gene 2 & male gene 1 & male gene 2 \\
\hline \multirow[t]{2}{*}{ Meseres } & Planemoides & $\begin{array}{l}2 \times \text { cenea } \\
2 \times \text { planemoides } \\
2 \times \text { hippocoonides }\end{array}$ & $\mathrm{Pl}$ & $h$ & $\mathrm{~h}$ & $\mathrm{c}$ \\
\hline & & $\begin{array}{l}3 \times \text { planemoides } \\
7 \times \text { hippocoonides }\end{array}$ & $\mathrm{Pl}$ & $\mathrm{h}$ & $\mathrm{h}$ & $\mathrm{h}(\mathrm{Pl} ?)$ \\
\hline \multirow[t]{2}{*}{ Tibullus } & Cenea & $\begin{array}{l}4 \times \text { cenea } \\
13 \times \text { hippoconides }\end{array}$ & $\mathrm{c}$ & $\mathrm{h}$ & h & $c(h ?)$ \\
\hline & & $\begin{array}{l}2 \times \text { cenea } \\
1 \times \text { hippocoonides }\end{array}$ & $\mathrm{c}$ & $\mathrm{h}$ & $h$ & $\mathrm{c} / \mathrm{h}$ \\
\hline \multirow[t]{22}{*}{ Cenea } & Cenea & $\begin{array}{l}16 \times \text { cenea } \\
1 \times \text { hippocoonides }\end{array}$ & $\mathrm{c}$ & $\mathrm{h}$ & $\mathrm{h}$ & $c ?$ \\
\hline & & $\begin{array}{l}24 \times \text { cenea } \\
3 \times \text { hippocoonides }\end{array}$ & c & $\mathrm{h}$ & $\mathrm{h}$ & $\mathrm{c} ?$ \\
\hline & & $\begin{array}{l}3 \times \text { cenea } \\
2 \times \text { leighi } \\
1 \times \text { hippocoonides }\end{array}$ & $\mathrm{c}$ & $\mathrm{h}$ & $\mathrm{h}$ & $\mathrm{L}$ \\
\hline & & $\begin{array}{l}21 \times \text { cenea } \\
2 \times \text { hippocoonides }\end{array}$ & $\mathrm{c}$ & $\mathrm{h}$ & h & $c ?$ \\
\hline & Trophonius & $\begin{array}{l}6 \times \text { cenea } \\
1 \times \text { trophonius }\end{array}$ & $\mathrm{T}$ & & c & \\
\hline & & $2 \times$ cenea & $\mathrm{T}$ & & $\mathrm{c}$ & \\
\hline & & $\begin{array}{l}22 \times \text { cenea } \\
4 \times \text { trophonius } \\
2 \times \text { leighi } \\
2 \times \text { hippocoonides }\end{array}$ & $T$ & $\mathrm{~h}$ & $\mathrm{c}$ & h? \\
\hline & & & $2^{\text {nd }}$ mating & & $\mathrm{L}$ & $\mathrm{h}$ ? \\
\hline & & $\begin{array}{l}9 \times \text { cenea } \\
4 \times \text { trophonius } \\
1 \times \text { leighi } \\
2 \times \text { hippocoonides } \\
\end{array}$ & $\mathrm{T}$ & $\mathrm{h}$ & c & h? \\
\hline & & & $2^{\text {nd }}$ mating & & $\mathrm{L}$ & $\mathrm{h}$ ? \\
\hline & & $\begin{array}{l}7 \times \text { cenea } \\
11 \times \text { hippocoonides }\end{array}$ & $\mathrm{T}$ & $\mathrm{h}$ & $\bar{c}$ & $\mathrm{~h}$ \\
\hline & & $\begin{array}{l}12 \times \text { cenea } \\
1 \times \text { trophonius } \\
5 \times \text { hippocoonides }\end{array}$ & $\mathrm{T}$ & $\mathrm{h}$ & $\mathrm{c}$ & $\mathrm{h}$ \\
\hline & & $\begin{array}{l}2 \times \text { cenea } \\
23 \times \text { trophonius }\end{array}$ & $\mathrm{T}$ & & $\mathrm{c}$ & \\
\hline & Hippocoonides & $\begin{array}{l}8 \times \text { cenea } \\
3 \times \text { trophonius } \\
3 \times \text { hippocoonides }\end{array}$ & $\mathrm{h}$ & $\mathrm{h}$ & c & h? \\
\hline & & & $2^{\text {nd }}$ mating & & $\mathrm{T}$ & $\mathrm{h}$ ? \\
\hline & & $13 \times$ cenea & $\mathrm{h}$ & $\mathrm{h}$ & c & $c(?)$ \\
\hline & & $\begin{array}{l}14 \times \text { cenea } \\
2 \times \text { trophonius } \\
4 \times \text { hippocoonides }\end{array}$ & $\mathrm{h}$ & $\mathrm{h}$ & c & $\mathrm{h} ?$ \\
\hline & & & $2^{\text {nd }}$ mating & & $\mathrm{T}$ & $\mathrm{h}$ ? \\
\hline & & $\begin{array}{l}17 \times \text { cenea } \\
8 \times \text { trophonius } \\
11 \times \text { hippocoonides } \\
\end{array}$ & $\mathrm{h}$ & $\begin{array}{l}\mathrm{h} \\
.\end{array}$ & $\mathrm{T}$ & h? \\
\hline & & & $2^{\text {nd }}$ mating & & $c$ & $\mathrm{~h}$ ? \\
\hline & & $\begin{array}{l}13 \times \text { cenea } \\
2 \times \text { hippocoonides }\end{array}$ & $\mathrm{h}$ & $\mathrm{h}$ & $\bar{c}$ & $\mathrm{~h}$ \\
\hline & Leighi & $1 \times$ hippocoonides & $\mathrm{L}$ & $\mathrm{h}$ & $\mathrm{h}$ & \\
\hline \multirow[t]{9}{*}{ Polytrophus } & Cenea & $\begin{array}{l}3 \times \text { cenea } \\
3 \times \text { hippocoonides }\end{array}$ & $\begin{array}{ll}\mathrm{c} \\
\mathrm{n}\end{array}$ & $\mathrm{h}$ & $\mathrm{h}$ & $h(c ?)$ \\
\hline & & $\mathrm{I} \times$ hippocoonides & $\mathrm{c}$ & $\mathrm{h}$ & $\mathrm{h}$ & \\
\hline & & $\begin{array}{l}12 \times \text { cenea } \\
2 \times \text { hippocoonides }\end{array}$ & $\bar{c}$ & $\mathrm{~h}$ & $\mathrm{~h}$ & $c(h ?)$ \\
\hline & & $\begin{array}{l}4 \times \text { cenea } \\
4 \times \text { hippocoonides }\end{array}$ & c & $\mathrm{h}$ & $\mathrm{h}$ & $h(c ?)$ \\
\hline & & $2 \times$ cenea & $\mathrm{c}$ & & & \\
\hline & & $\begin{array}{l}1 \times \text { cenea } \\
1 \times \text { hippocoonides }\end{array}$ & c & $\mathrm{h}$ & $\mathrm{h}$ & $h / c$ \\
\hline & & $\begin{array}{l}9 \times \text { cenea } \\
4 \times \text { hippocoonides }\end{array}$ & c & $\mathrm{h}$ & $\mathrm{h}$ & $\mathrm{c} / \mathrm{h}$ \\
\hline & & $18 \times$ cenea & $\mathrm{c}$ & & & \\
\hline & & $\begin{array}{l}6 \times \text { cenea } \\
2 \times \text { salaami } \\
1 \times \text { hippocoonide }\end{array}$ & $\mathrm{c}$ & $\mathrm{h}$ & $\mathbf{s}$ & $\mathrm{h}$ \\
\hline
\end{tabular}


Appendix 4 Analysis of data from Ford (1936)

\begin{tabular}{|c|c|c|c|c|c|c|}
\hline & & $\begin{array}{l}6 \times \text { cenea } \\
1 \times \text { hippocoonides }\end{array}$ & c & h & $\mathrm{h}$ & $\mathrm{c}(\mathrm{h} ?)$ \\
\hline & & $\begin{array}{l}1 \times \text { cenea } \\
4 \times \text { trophonius }\end{array}$ & $\mathrm{c}$ & & $T$ & \\
\hline & & $20 \times$ cenea & c & & & \\
\hline & & $8 \times$ cenea & $c$ & & & \\
\hline & & $\begin{array}{l}3 \times \text { cenea } \\
2 \times \text { hippocoonides }\end{array}$ & $\mathrm{c}$ & $\mathrm{h}$ & $h$ & $\mathrm{c} / \mathrm{h}$ \\
\hline & & $\begin{array}{l}7 \times \text { cenea } \\
2 \times \text { hippocoonides }\end{array}$ & $\mathrm{c}$ & h & $\mathrm{h}$ & $\mathrm{c}(\mathrm{h} ?)$ \\
\hline & & $2 \times$ cenea & $\mathrm{c}$ & & & \\
\hline & & $\begin{array}{l}24 \times \text { cenea } \\
1 \times \text { salaami } \\
4 \times \text { hippocoonides } \\
\end{array}$ & c & $\mathrm{h}$ & s & $\mathrm{h}$ \\
\hline & & $7 \times$ trophonius & $c$ & & $\mathrm{~T}$ & \\
\hline & & $\begin{array}{l}1 \times \text { cenea } \\
2 \times \text { trophonius } \\
1 \times \text { hippocoonides }\end{array}$ & c & $\mathbf{h}$ & $T$ & $\mathrm{~h}$ \\
\hline & & $\begin{array}{l}1 \times \text { cenea } \\
11 \times \text { hippocoonides }\end{array}$ & c & h & $\mathrm{h}$ & $h(c ?)$ \\
\hline & & $\begin{array}{l}3 \times \text { cenea } \\
6 \times \text { hippocoonides }\end{array}$ & $\mathrm{c}$ & h & $\mathrm{h}$ & $h(c ?)$ \\
\hline & & $8 \times$ cenea & $c$ & & & \\
\hline & & $\begin{array}{l}3 \times \text { cenea } \\
1 \times \text { hippocoonides }\end{array}$ & $\mathrm{c}$ & h & $\mathrm{h}$ & $\mathrm{c} / \mathrm{h}$ \\
\hline & & $\begin{array}{l}15 \times \text { cenea } \\
1 \times \text { hippocoonides }\end{array}$ & c & h & $\mathrm{h}$ & $\mathrm{c}(\mathrm{h} ?)$ \\
\hline & & $\begin{array}{l}9 \times \text { cenea } \\
5 \times \text { hippocoonides }\end{array}$ & $\mathrm{c}$ & $\mathrm{h}$ & $\mathrm{h}$ & $\mathrm{c} / \mathrm{h}$ \\
\hline & & $\begin{array}{l}9 \times \text { cenea } \\
1 \times \text { hippocoonides }\end{array}$ & c & $\mathrm{h}$ & $\mathrm{h}$ & $c(h ?)$ \\
\hline & & $\begin{array}{l}4 \times \text { cenea } \\
7 \times \text { salaami } \\
4 \times \text { hippocoonides } \\
\end{array}$ & $\mathrm{c}$ & $\mathrm{h}$ & $\mathrm{s}$ & $\mathrm{h}$ \\
\hline & & $\begin{array}{l}6 \times \text { cenea } \\
7 \times \text { hippocoonides }\end{array}$ & $\mathrm{c}$ & $\mathrm{h}$ & h & $h(c ?)$ \\
\hline & & $\begin{array}{l}13 \times \text { cenea } \\
1 \times \text { salaami } \\
4 \times \text { hippocoonides }\end{array}$ & $\mathrm{c}$ & $\mathrm{h}$ & $\mathrm{h}$ & $\mathrm{s}$ \\
\hline & & $4 \times$ cenea & $c$ & & & \\
\hline & & $\begin{array}{l}5 \times \text { cenea } \\
8 \times \text { trophonius }\end{array}$ & $\bar{c}$ & & $T$ & \\
\hline & Trophonius & $\begin{array}{l}18 \times \text { cenea } \\
12 \times \text { trophonius } \\
12 \times \text { hippocoonides }\end{array}$ & $T$ & $\mathrm{~h}$ & c & $\mathrm{h}$ \\
\hline & & $\begin{array}{l}8 \times \text { cenea } \\
10 \times \text { trophonius } \\
27 \times \text { salaami } \\
19 \times \text { hippocoonides }\end{array}$ & $T$ & $\mathrm{~h}$ & $\mathrm{c}$ & $\mathrm{h}$ \\
\hline & & & $2^{\text {nd }}$ mating & & $\mathrm{s}$ & h \\
\hline & & $\begin{array}{l}\times \text { cenea } \\
6 \times \text { trophonius } \\
5 \times \text { hippocoonides }\end{array}$ & $T$ & $\mathrm{~h}$ & $\mathrm{c}$ & h \\
\hline & & $\begin{array}{l}6 \times \text { trophonius } \\
5 \times \text { hippocoonides }\end{array}$ & $\mathrm{T}$ & h. & $\mathrm{h}$ & $\mathrm{h}(\mathrm{T}$ ?) \\
\hline & Salaami & $\begin{array}{l}1 \times \text { cenea } \\
1 \times \text { salaami } \\
1 \times \text { hippocoonides }\end{array}$ & $s$ & $\mathrm{~h}$ & $\mathrm{c}$ & $\mathrm{h}$ \\
\hline & & $\begin{array}{l}6 \times \text { salaami } \\
7 \times \text { hippocoonides }\end{array}$ & $\mathrm{s}$ & $\mathrm{h}$ & $\mathrm{h}$ & $\mathrm{h}(\mathrm{s} ?)$ \\
\hline & & $\begin{array}{l}6 \times \text { cenea } \\
7 \times \text { salaami } \\
\end{array}$ & $\mathrm{s}$ & & c & \\
\hline & & $18 \times$ salaami & $s$ & $s$ & $s / \mathrm{h}$ & $s / h$ \\
\hline & & $\begin{array}{l}2 \times \text { cenea } \\
8 \times \text { salaami } \\
13 \times \text { hippocoonides } \\
\end{array}$ & $s$ & $\mathrm{~h}$ & $\mathrm{c}$ & $\mathrm{h}$ \\
\hline & & $\begin{array}{l}2 \times \text { salaami } \\
2 \times \text { hippocoonides }\end{array}$ & $\mathrm{s}$ & $\mathrm{h}$ & $\mathrm{h}$ & $h(s ?)$ \\
\hline & & $\begin{array}{l}2 \times \text { salaami } \\
1 \times \text { hippocoonides }\end{array}$ & $\mathrm{s}$ & $\mathrm{h}$ & $\mathrm{h}$ & h (s?) \\
\hline & & $\begin{array}{l}2 \times \text { trophonius } \\
5 \times \text { salaami } \\
1 \times \text { hippocoonides }\end{array}$ & $s$ & $\mathrm{~h}$ & $T$ & $\mathrm{~h}$ \\
\hline & & $\begin{array}{l}2 \times \text { salaami } \\
2 \times \text { hippocoonides }\end{array}$ & $\mathrm{s}$ & $\mathrm{h}$ & h & $h(s ?)$ \\
\hline
\end{tabular}




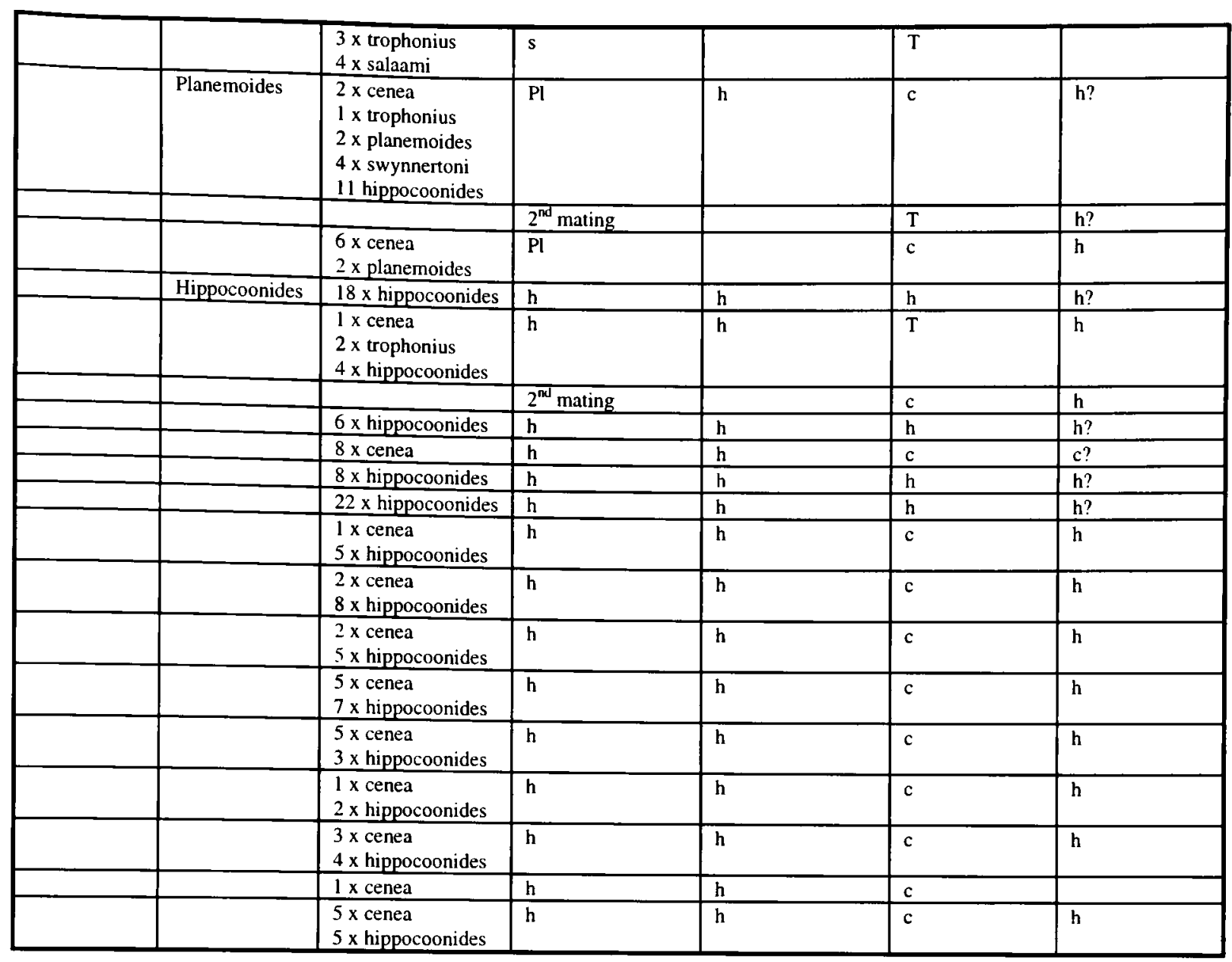

Table A4-1 Summary of the genes carried by parents of broods from wild-caught females, from data gathered by Ford (1936)

\section{Proportions of morphs in the races}

Ford also published estimates of the proportions of the morphs as assessed by 'random sampling' in various areas (for references, again see Ford, 1936; data also in Appendix 1). For the races above, these are (listed in order of gene dominance):

\begin{tabular}{|c|c||c|c||c|c|}
\hline \multicolumn{2}{|c||}{ Race Cenea } & \multicolumn{2}{c||}{ Race Meseres } & \multicolumn{2}{c|}{ Race Polytrophus } \\
\hline Trophonius & $4 \%$ & Trophonius & $5 \%$ & Trophonius & $12 \%$ \\
\hline Leighi & $1 \%$ & Planemoides & $21 \%$ & Poultoni (Salaami) & $10 \%$ \\
\hline Cenea & $85 \%$ & Salaami & $7 \%$ & Cenea & $43 \%$ \\
\hline Natalica & very rare & Leighi & very rare & Hippocoonides & $35 \%$ \\
\hline Hippocoonides & $10 \%$ & Cenea & $7 \%$ & & \\
\hline & & Hippocoonides & $60 \%$ & & \\
\hline
\end{tabular}

Table A4-2 The proportions of morphs recorded for populations of Papilio dardanus

Exact proportions of the morphs in the mainland population of race tibullus are not recorded. 


\section{Assessment of assortative mating (Chapter 5)}

Given the proportions of the various phenotypes (morphs) it is possible to calculate the frequency of each gene in each race (since the frequency of the h gene is simply the square root of the proportion of hippocoonides morphs, and the others can be calculated from this):

\begin{tabular}{|c|c||c|c||c|c|}
\hline \multicolumn{2}{|c||}{ Race Cenea } & \multicolumn{2}{c||}{ Race Meseres } & \multicolumn{2}{c|}{ Race Polytrophus } \\
\hline $\mathrm{T}$ & 0.020 & $\mathrm{~T}$ & 0.0253 & $\mathrm{~T}$ & 0.062 \\
\hline $\mathrm{L}$ & 0.005 & $\mathrm{Pl}$ & 0.114 & $\mathrm{~s}$ & 0.055 \\
\hline $\mathrm{c}$ & 0.658 & $\mathrm{~s}$ & 0.042 & $\mathrm{c}$ & 0.292 \\
\hline $\mathrm{h}$ & 0.316 & $\mathrm{c}$ & 0.0439 & $\mathrm{~h}$ & 0.592 \\
\hline & & $\mathrm{h}$ & 0.775 & & \\
\hline
\end{tabular}

Table A4-3 Calculated gene frequencies in various races of Papilio dardanus

Therefore it is possible to test whether or not the gene frequencies found in the males mating with a certain morph deviate significantly from these expected values for each race. The fact that dominant genes often cover the effect of others makes this analysis difficult. For race meseres, there is not enough data to analyse statistically, and for race tibullus the gene frequencies are not known, so only the data for races cenea and polytrophus could be analysed. The observed gene frequencies in the males for each morph are shown in Table A4-4.

\begin{tabular}{|c|c|c|c|c|c|c|c|}
\hline Race & Morph & T & Pl & S & L & c & h \\
\hline Cenea & cenea & 0 & - & & 0.125 & 0.375 & 0.5 \\
\hline & trophonius & 0 & - & & 0.154 & 0.385 & 0.462 \\
\hline & hippocoonides & 0.188 & & & 0 & 0.375 & 0.438 \\
\hline & leighi & & & & & & \\
\hline Polytrophus & cenea & 0.111 & & 0 & & 0.25 & 0.75 \\
\hline & trophonius & 0 & 0 & 0.1 & & 0.3 & 0.6 \\
\hline & salaami & 0.667 & 0 & 0.056 & & 0.167 & 0.667 \\
\hline & planemoides & 0.167 & 0 & 0 & & 0.333 & 0.5 \\
\hline & hippocoonides & 0.032 & 0 & 0 & & 0.387 & 0.581 \\
\hline
\end{tabular}

Table A4-4 The observed gene frequencies in males mating with each morph in races cenea and polytrophus

Unfortunately, most of the data sets are too small to analyse statistically. Two can be analysed using a chi-squared test with some degree of certainty - race polytrophus, cenea and hippocoonides broods. For these, the data for salaami and trophonius need to be combined to make large enough expected values. For both sets the observed values are not significantly different from the expected proportions in the population (cenea: $\mathrm{p}=0.56, \chi^{2}=1.18, \mathrm{n}=40$; hippocoonides: $\mathrm{p}=0.24, \chi^{2}$ $=2.87, \mathrm{n}=31$ ). It therefore appears that there is no assortative mating

One further test that can be done involves analysing the genes carried by the females. They are themselves the offspring of wild pairings, and many of their genotypes are fully known - more so 
than the males. One outcome of assortative mating is that it results in more homozygotes than would otherwise be expected.

The expected ratio of homozygotes to heterozygotes for races polytrophus and cenea can be calculated from the frequencies of the genes, shown in Table A4-3. To do this, it is necessary to calculate the expected frequencies of each possible genotype (the frequency of one gene multiplied by the frequency of the other). These expected frequencies are shown in Table A4-5.

\begin{tabular}{|c|c||c|c|}
\hline Race polytrophus & Expected frequency & Race cenea & Expected frequency \\
\hline $\mathrm{hc}$ & 0.346 & $\mathrm{hc}$ & 0.416 \\
\hline $\mathrm{hs}$ & 0.065 & $\mathrm{hL}$ & 0.003 \\
\hline $\mathrm{hT}$ & 0.073 & $\mathrm{hT}$ & 0.013 \\
\hline $\mathrm{cs}$ & 0.032 & $\mathrm{cL}$ & 0.007 \\
\hline $\mathrm{cT}$ & 0.036 & $\mathrm{cT}$ & 0.026 \\
\hline sT & 0.007 & $\mathrm{LT}$ & 0.0002 \\
\hline $\mathrm{TT}$ & 0.004 & $\mathrm{TT}$ & 0.0004 \\
\hline $\mathrm{ss}$ & 0.003 & $\mathrm{LL}$ & 0.00003 \\
\hline $\mathrm{cc}$ & 0.085 & $\mathrm{cc}$ & 0.433 \\
\hline $\mathrm{hh}$ & 0.350 & $\mathrm{hh}$ & 0.100 \\
\hline
\end{tabular}

Table A4-5 The expected frequencies of each genotype in races cenea and polytrophus

For race polytrophus, the genotypes of 39 females are known with relative certainty, whilst in race cenea, 14 are known (see Table A4-1). This gives expected ratios of heterozygotes:homozygotes in each race to be:

Race polytrophus

$27.41: 21.69$

Race cenea

$6.51: 7.47$

The observed ratios are:

Race polytrophus

$33: 16$

Race cenea

$9: 5$

A binomial test on each of these results indicates that neither observed ratio deviates significantly from the expected (polytrophus: $p=0.919, n=49$; cenea: $p=0.152, n=14$ ). Therefore it appears that the males are not choosing female morphs assortatively on the basis of the genes that they themselves carry.

\section{Assessment of the frequency of multiple matings (Chapter 7)}

Some wild-caught females had offspring which must have had multiple parentage (see Table A4-1). However, many which had mated more than once would be expected to have offspring which 
would not show this - particularly when one male had genes which were either exactly the same, or recessive to those of the other.

Considering the females which can be seen to have mated more than once, there is a different pattern in different races and morphs (shown in Table A4-6)

\begin{tabular}{|c|c|c|c|}
\hline Race & Morph & No. mated once & No. mated twice \\
\hline Cenea & cenea & 4 & 0 \\
\hline & trophonius & 5 & 2 \\
\hline & hippocooonides & 2 & 3 \\
\hline & leighi & 1 & 0 \\
\hline & cenea & 31 & 0 \\
\hline Polytrophus & trophonius & 3 & 1 \\
\hline & salaami & 10 & 0 \\
\hline & planemoides & 1 & 1 \\
\hline & hippocoonides & 14 & 1 \\
\hline
\end{tabular}

Table A4-6 The frequencies of multiple mating in different morphs and races as assessed from the data of Ford (1936)

Overall, the two races show a mean frequency of double matings to be $10 \%$, and as explained previously, this can be taken to be a minimum value. Race cenea shows a minimum double mating frequency of $41 \%$, which is surprisingly high as the real frequency is likely to be much higher. Race polytrophus, on the other hand, shows a minimum frequency of $5.1 \%$, which is quite low. The fact that the bottom recessive hippocoonides gene is so common in race polytrophus (frequency $=0.592$ ) means that many matings will involve hh males. Therefore many second matings will not be recognisable, as the genes from one male will be the same as or dominant over the hh male. By contrast, in race cenea, the more dominant cenea gene is much more frequent (frequency $=0.658$ ), and so the common gene $\mathrm{c}$ will show up in broods with double parentage much more frequently. This data therefore suggests that, at least in some races, the frequency of double matings might be quite high.

\section{$\underline{\text { References }}$}

Ford, E.B. 1936. The genetics of Papilio dardanus Brown (Lep.) Trans. R. Ent. Soc. London 85, 435-465.

Clarke, C.A. \& Sheppard, P.M. 1959. The genetics of Papilio dardanus, Brown. I race cenea from South Africa. Genetics 44: 1347-1358.

Clarke, C.A. \& Sheppard, P.M. 1960a. The genetics of Papilio dardanus, Brown. 11 races dardanus, polytrophus, meseres and tibullus. Genetics 45 : 439-457.

Clarke, C.A. \& Sheppard, P.M. 1960b. The genetics of Papilio dardanus, Brown. III race antinorii from Abyssinia and race meriones from Madagascar. Genetics 45: 683-698. 
Clarke, C.A. \& Sheppard, P.M. 1962. The genetics of Papilio dardanus, Brown. IV data on race ochracea, race flavicornis, and further information on races polytrophus and dardanus. Genetics 47 : 909-920.

Clarke, C.A. \& Sheppard, P.M. 1963. Interactions between major genes and polygenes in the determination of mimetic patterns of Papilio dardanus. Evolution 17, 404-413. 


\section{Appendix Five}

\section{Analysis of data from Cook et al. (1994)}

\section{Assessment of whether or not the data is additive}

Cook et al. (1994) carried out pairwise choice tests on Papilio dardanus (race tibullus on the island of Pemba) by pinning out pairs of dead butterflies and counting the number of first approaches by males which were made to each butterfly. They used all three females morphs present (hippocoonides, trimeni, and lamborni), and also compared each of these with pinned-out males. In addition they compared hippocoonides and its model Amauris niavius, males with faded males, and trimeni and lamborni morphs with faded trimeni morphs. These comparisons are not used in the analysis, as the faded specimens and Amauris niavius were not compared in every possible combination.

The number of approaches recorded by Cook et al. for each comparison used in this analysis therefore is:

\begin{tabular}{|c|c|c|c|c|}
\hline $\begin{array}{c}\text { Number of approaches to } \rightarrow \\
\text { When compared with } \downarrow\end{array}$ & hippocoonides & trimeni & lamborni & male \\
\hline hippocoonides & - & 11 & 7 & 11 \\
\hline trimeni & 54 & - & 16 & 30 \\
\hline lamborni & 42 & 40 & - & 43 \\
\hline male & 41 & 48 & 26 & - \\
\hline
\end{tabular}

Table A5-1 Numbers of approaches to each morph in pair-wise choice comparisons by Cook et al.

Converting these to percentages gives:

\begin{tabular}{|c|c|c|c|c|}
\hline $\begin{array}{c}\text { \%of approaches to } \rightarrow \\
\text { When compared with } \downarrow\end{array}$ & hippocoonides & trimeni & lamborni & male \\
\hline hippocoonides & - & 16.9 & 14.3 & 21.2 \\
\hline trimeni & 83.1 & - & 28.6 & 38.5 \\
\hline lamborni & 85.7 & 71.4 & - & 62.3 \\
\hline male & 78.8 & 61.5 & 37.7 & - \\
\hline
\end{tabular}

Table A5-2 Percentages of approaches to each morph in pair-wise comparisons by Cook et al. 
For the data to be additive (i.e. for $\mathrm{A}: \mathrm{B}$ and $\mathrm{B}: \mathrm{C}$ to predict $\mathrm{A}: \mathrm{C}$ ), the ratio of $\mathrm{A}: \mathrm{B}$ multiplied by the ratio of $B: C$ multiplied by the ratio of $A: C$ must equal 1. Therefore each combination of three morphs (including the male) can be tested for consistency with the data being additive:

hippocoonides:trimeni $\mathrm{x}$ trimeni:lamborni $\mathrm{x}$ hippocoonides:lamborni $=2.03$

hippocoonides:trimeni $\mathrm{x}$ trimeni:male $\mathrm{x}$ hippocoonides:male $=2.1$

hippocoonides:lamborni $\mathrm{x}$ lamborni:male $\mathrm{x}$ hippocoonides:male $=0.98$

trimeni:lamborni $\mathrm{x}$ lamborni:male $\mathrm{x}$ trimeni:male $=0.94$

From this analysis it can be seen that all the data, excluding that found for hippocoonides versus trimeni, can be taken to be additive. It is possible that the hippocoonides versus trimeni data is a statistical anomaly, or that this particular pairing causes a more complex effect on the males' preferences.

If the data as a whole is taken to represent the underlying preferences of the males, the expected preferences in the choice test between hippocoonides and trimeni can be calculated (by setting the top two equations above to one and substituting the values of the other ratios).

This gives the new percentage preferences for hippocoonides and trimeni to be $70.59 \%$ and $29.41 \%$ respectively.

\section{Calculation of the percentage preferences for each morph}

Having taken the data to be additive, and calculated new values for the preferences of hippocoonides versus trimeni in order to make the data self-consistent, it is now possible to calculate the overall percentage preferences for each morph represented by the data.

The preference for hippocoonides (h) over lamborni $(1)$ and trimeni $(t)$ can be represented as:

$$
\frac{h}{t+1}=\frac{1}{t / h+1 / h}
$$

Since $t / h$ and $1 / h$ are known, the overall preference can be calculated. This can be done for each morph (and to check that these are correct, they should all add up to 100\%).

This gives the percentage preferences for each morph to be: 
hippocoonides: 63.16

trimeni: $\quad 26.32$

lamborni: $\quad 10.53$

\section{Calculation of the probability of a male mating twice}

If it is assumed that males only mate either once or twice (no broods from wild females reported by Ford (1936) indicate that a female had mated more than twice and so it seems a reasonable assumption that it is rare for males to mate more than twice), then the probability of a male choosing a hippocoonides $(\mathrm{h})$ female in the mate choice experiments can be written as:

$\mathrm{p}($ choosing $\mathrm{h}$ in experiment $)=\mathrm{p}($ this is 1 st mating $) \times \mathrm{p}\left(\right.$ choosing $\mathrm{h}$ in experiment on $1^{\text {st }}$ mating $)$ $+\mathrm{p}$ (this is $2^{\text {nd }}$ mating) $\times \mathrm{p}$ (choosing $\mathrm{h}$ in experiment on $2^{\text {nd }}$ mating)

The probability that this is the first mating is taken to be $1-p$ (this is $2^{\text {nd }}$ mating) since males are assumed to mate a maximum of two times.

If the males mate randomly on their first mating, as suggested by the results of Experiment 51 , then the probability of mating with the hippocoonides female in the experiment is $1 / 3$, as each morph was presented an equal number of times.

The probability that a male chooses hippocoonides on the second mating can be broken down:

$\begin{aligned} \mathrm{p}\left(\text { choosing h on } 2^{\text {nd }} \text { mating }\right)= & \mathrm{p}\left(\text { chose } \mathrm{h} \text { on } 1^{\text {st }} \text { mating }\right) \times \mathrm{p}(\text { choosing same morph }) \\ & +\mathrm{p}\left(\text { chose } \mathrm{t} \text { on } 1^{\text {st }} \text { mating }\right) \times \mathrm{p}(\text { choosing } \mathrm{h} \text { if chose } \mathrm{t}) \\ & +\mathrm{p}\left(\operatorname{chose} 1 \text { on } 1^{\text {st }} \text { mating }\right) \times \mathrm{p}(\text { choosing } \mathrm{h} \text { if chose } \mathrm{l})\end{aligned}$

Again, if the males mate randomly when naive, then the probability of choosing each morph on the first mating is equal to its proportion in the population (which was estimated by Cook et al.). If it is assumed that if a male does not choose the same morph on his second mating as on his first then he chooses randomly between the other two, then the $p$ (choosing $h$ if chose $t$ ) and $p$ (choosing $h$ if chose 1) are both 0.5 (1-p(choosing same morph).

Thus: 
$\mathrm{p}\left(\right.$ choosing $\mathrm{h}$ on $2^{\text {nd }}$ mating $)=\mathrm{p}(\mathrm{h}$ in population $) \times \mathrm{p}($ choosing same morph $)$

$+\mathrm{p}(\mathrm{t}$ in population $) \times 0.5(1-\mathrm{p}$ (choosing same morph))

$+\mathrm{p}(1$ in population $) \times 0.5(1-\mathrm{p}($ choosing same morph $))$

And overall:

$\mathrm{p}($ choosing $\mathrm{h}$ in experiment $)=\left[1-\mathrm{p}\left(\right.\right.$ this is $2^{\text {nd }}$ mating $\left.)\right] \times 1 / 3$

$+\mathrm{p}$ (this is $2^{\text {nd }}$ mating) $x[\mathrm{p}(\mathrm{h}$ in population $) \mathrm{x}$ (choosing same morph)

$+\mathrm{p}(\mathrm{t}$ in population $) \times 0.5(1-\mathrm{p}($ choosing same morph $))$

$+\mathrm{p}(1$ in population $) \times 0.5(1-\mathrm{p}($ choosing same morph $))]$

Since the $\mathrm{p}$ (choosing $\mathrm{h}$ in the experiment) has been calculated from the data of Cook et al., and they also calculated the proportions of each morph in each population, this equation contains only two unknowns - the probability that the males mate twice and the probability of mating with the same morph twice. By substituting in the value for the probability of mating with the same morph on a second mating obtained from Experiment 5-2 it is possible to calculate the proportion of males which must have mated previously to give the results obtained by Cook et al. for each of the morphs.

\begin{tabular}{|c|c|c|}
\hline Parameter & Value & Source \\
\hline proportion hippocoonides (h) & 0.8 & Cook et al. (1994) \\
\hline proportion trimeni ( $\mathrm{t})$ & 0.16 & Cook et al. (1994) \\
\hline proportion lamborni (l) & 0.04 & Cook et al. (1994) \\
\hline probability choosing h in expt. & 0.63 & Calculated from Cook et al. (1994) \\
\hline probability choosing t in expt. & 0.26 & Calculated from Cook et al. (1994) \\
\hline probability choosing l in expt. & 0.1 & Calculated from Cook et al. (1994) \\
\hline probability choosing same morph & 0.75 & Experiment 5-2 \\
\hline
\end{tabular}

Table A5-3 Parameters and their values used to calculate the probability of mating twice from the results of Cook et al. and Experiment 5-2

This substitution gives the proportion of males which must have already mated to give the results of Cook et al.'s choice tests for each morph:

hippocoonides: $102.26 \%$

trimeni: $\quad 64.78 \%$

lamborni: $\quad 124.4 \%$

Giving an average of $97.14 \%$.

It is possible that the probability of choosing the same morph twice recorded in Experiment 52 is inaccurate, since this value is based on only 8 data points. However, if this value is treated as 
'unknown' in the equations above, it is possible to solve the three equations simultaneously, to calculate the proportions of males expected to have mated already solely from the data of Cook et al. The easiest way in which to do this is to create a surface of the error between the results predicted by the three equations with varying values for the probability of mating twice and the probability of choosing the same morph twice, and to find the minimum value on the surface, representing the values of these two parameters which give the closest prediction to the observed results.

The surface produced is shown in Figure A5-1. From these results a minimum value for the error can be found, and it is where both the probability of mating twice and the probability of choosing the same morph on both those matings are 0.84 .

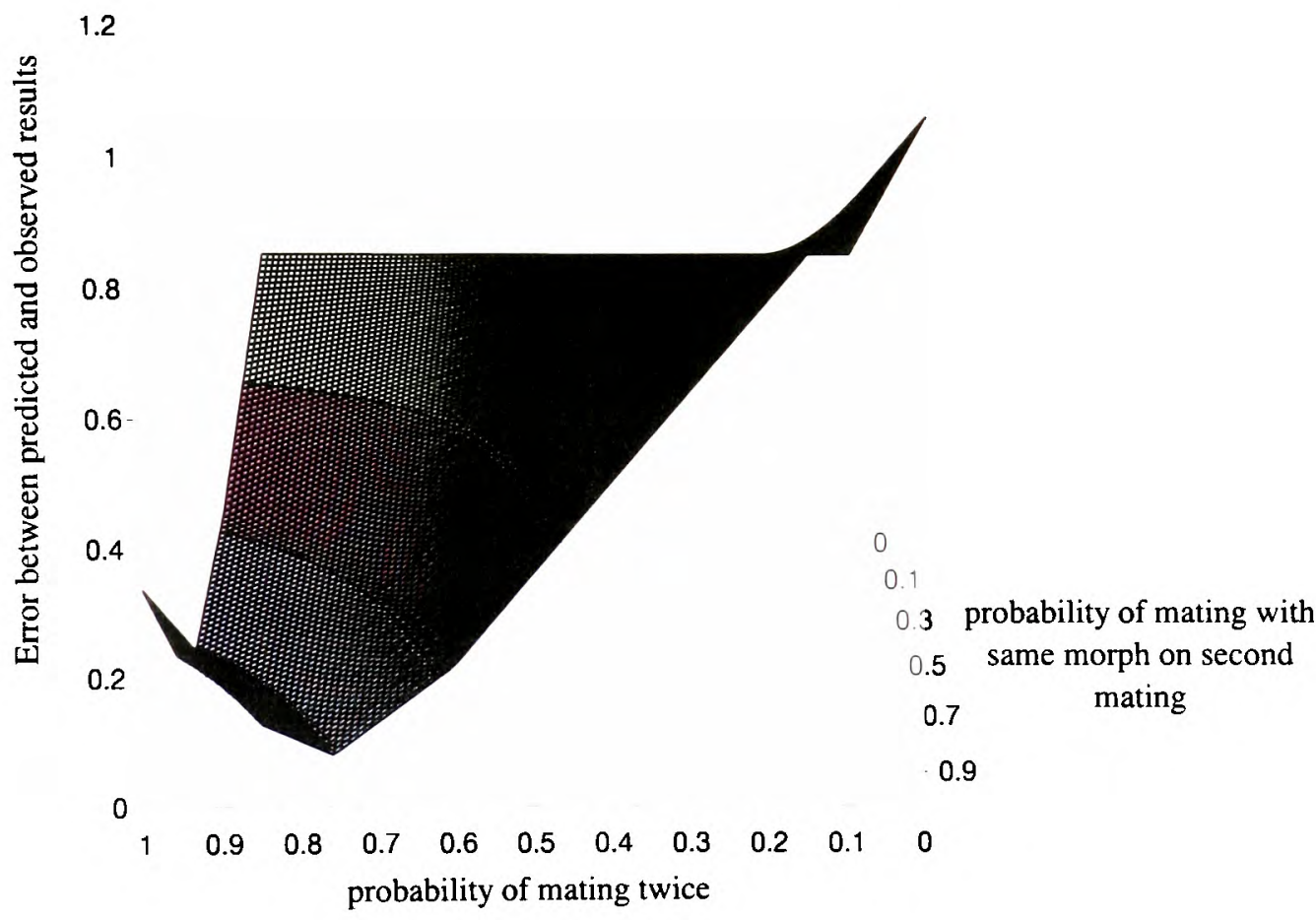

Figure A5-1 Surface plot showing the changes in error between the observed data of Cook et al. and the predicted model of learning with changing values of the probability of mating twice and the probability of mating with the same morph twice

\section{$\underline{\text { References }}$}

Cook, S.E., Vernon, J.G., Bateson, M., Guilford, T. 1994. Mate choice in the polymorphic African swallowtail butterfly, Papilio dardanus: male-like females may avoid sexual harassment. Anim. Behav. 47: 389-397. 


\section{Appendix Six}

\section{Program listing}

\section{Program 1}

/*

*A C program to calculate the p-value of a certain result in Experiment 5-4, written by John Alden"

*

*

*/

\#include < math.h>

\#include <stdio.h>

\#define MAX 100

int power[MAX+1];

double sum[MAX+1],p[MAX];

void main(void)

\{

/*Define variables */

char filename[100],description[200];

register int $\mathrm{j}$;

register long $i$;

FILE *fp;

int nexp,nobs;

int idx;

double temp,cumul1[MAX+1],cumul2[MAX+1];

\section{$1 *$ Read file *}

printf("InEnter filename:"); scanf("\%s",filename);

$\mathrm{fp}=$ fopen (filename, "rt");

if $(\mathrm{fp}==\mathrm{NULL})\{$ printf("Couldn't open file !!!" ); exit(1);\}

fgets(description,200,fp);

fscanf(fp,"\%d",\&nexp); fgets(filename,100,fp);

fscanf(fp,"\%d",\&nobs); fgets(filename, 100,fp);

fgets(filename, $100, f p$ );

for $(j=0 ; j<=n \exp ; j++)$ fscanf(fp,"\%lf",p+j);

fclose(fp); 


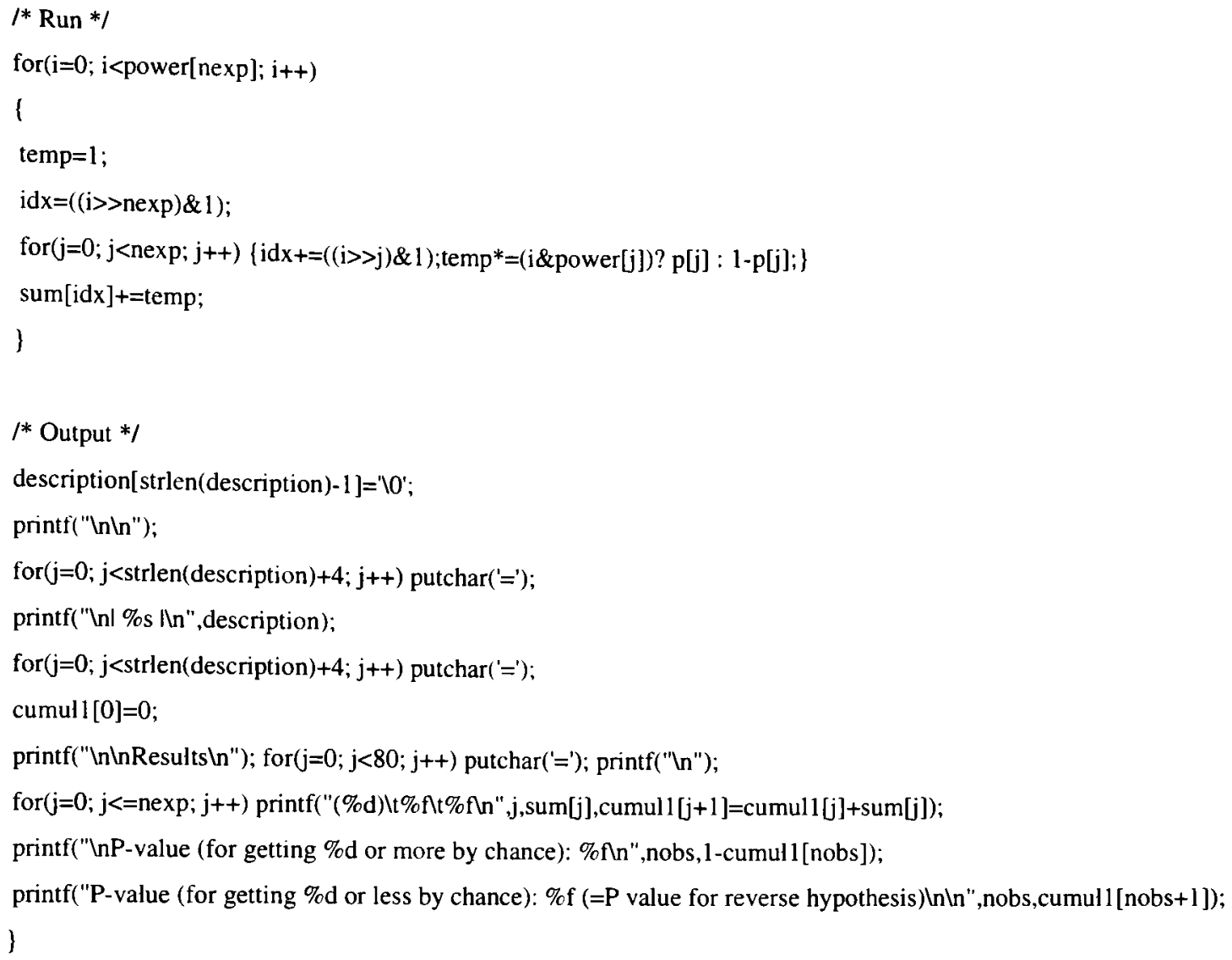

\section{Program 2}

\section{/*}

* A C program to simulate the population dynamics of three morphs in Papilio dardanus written by Alex

\section{Freeman}

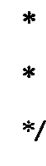

\#include <stdio.h>

\#include < math.h>

\section{/* Declare subroutines */}

void PrintHeadings();

void Display(int GEN, const char *comment, double Phh, double Phc, double Pcc, double Pht, double Pct, double $P t t)$

double sigmoid(double $\mathrm{x}$, double a, double lxmin, double lxmax, double lymin, double lymax);

double predation(double Pmodel, double Pmimic, double n_memory);

void Normalise (double ${ }^{*} \mathrm{a}$, double ${ }^{*} \mathrm{~b}$, double ${ }^{*} \mathrm{c}$, double ${ }^{*}$, double ${ }^{*}$, double ${ }^{*} \mathrm{f}$ );

void solve_GF(double H,double C,double T, double ${ }^{*} \mathrm{~h}$, double ${ }^{*} \mathrm{c}$, double ${ }^{*} \mathrm{t}$ );

void solve_MF(double ${ }^{*} \mathrm{H}$, double ${ }^{*} \mathrm{C}$, double ${ }^{*} \mathrm{~T}$, double $\mathrm{h}$, double $\mathrm{c}$, double $\mathrm{t}$ );

double solve_quad(double a, double b, double c); 
void main(void)

\{

/* declare variables */

double PH, PC, PT;

/* frequency hippo, cenea, lamborni - calculated */

double $\mathrm{Phh}, \mathrm{Phc}, \mathrm{Pcc}, \mathrm{Pht}, \mathrm{Pct}, \mathrm{Ptt} ; \quad$ * frequency of each genotype in 1st generation - calculated */

double Ihh, Ihc, Icc, lht, lct, Itt; $\quad / *$ frequency of each genotype in next generation before predation */

double $\mathrm{Ph}, \mathrm{Pc}, \mathrm{Pt} ; \quad \quad / *$ frequency of each gene in population - defined $* /$

double Mhh, Mhc, Mcc, Mht, Mct, Mtt; I* probability of mating with each genotype - calculated*/

double Ml hh, M1hc, Mlcc, MIht, M1ct, M1tt; /* probability of mating with each genotype on first mating calculated $*$

double $\mathrm{CH}, \mathrm{CC}, \mathrm{CT}$;

/* probability of choosing each morph - defined*/

double $\mathrm{F}, \mathrm{N}$;

/* probability of follow-up mating or not a follow-up mating - defined*/

double $\mathrm{S}, \mathrm{D}$;

/* probability of choosing same or different morph on follow-up - defined*/

int GEN;

/* generation counter */

double N_MEM, Pencounter;

/* Predator Memory function and encounter likelihood */

double RmodelH,RmodelC,RmodelT;

/* Ratios of model to mimics [at start] */

double AmodelH,AmodelC,AmodelT;

$f^{*}$ Abundance of models relative to dardanus morphs -

calculated*/

$\mathrm{GEN}=0$

/*probabilities for follow-up matings */

$\mathrm{F}=0.59 ; / /$ number of females mating more than once

$\mathrm{N}=1-\mathrm{F}$;

$\mathrm{S}=0.75 ; / /$ probability of choosing same morph twice

$\mathrm{D}=1-\mathrm{S}$;

/* morph proportions */

$\mathrm{PH}=70 . /(70+25+8) ; \quad / /$ These are Ford's data for race meseres

$\mathrm{PC}=25 . /(70+25+8)$;

$\mathrm{PT}=8 . /(70+25+8)$;

/* probabilities of choosing each morph on first mating - can be varied*/

$\mathrm{CH}=\mathrm{CC}=\mathrm{CT}=1$;

// Relative abundance of mimics \& models $=>$ predation

Pencounter=0.95; // Chance of encountering a predator before mating (VARIABLE)

N_MEM=40; $\quad$ // Memory function of predators (VARIABLE)

RmodelH=104/70.; // Model abundance (initially) - data from Ford for meseres

$\mathrm{RmodelC}=232 / 8$;

RmodelT=781/25;

/* initial gene proportions */

solve_GF(CH,CC,CT,\&Ph,\&Pc,\&Pt); 


\section{/* calculate genotype proportions from gene proportions */}

$\mathrm{Phh}=\mathrm{Ph} * \mathrm{Ph}$;

$\mathrm{Phc}=2 * \mathrm{Ph}^{*} \mathrm{Pc}$;

$\mathrm{Pcc}=\mathrm{Pc} * \mathrm{Pc}$;

$\mathrm{Pht}=2 * \mathrm{Ph}^{*} \mathrm{Pt}$;

$\mathrm{Pct}=2 * \mathrm{Pc} * \mathrm{Pt}$;

$\mathrm{Ptt}=\mathrm{Pt} * \mathrm{Pt}$

\section{/*print out initial proportions */}

PrintHeadings( ):

Display(0,"Initial proprtions",Phh,Phc,Pcc,Pht,Pct,Pt);

//

\section{// Generation LOOP}

//

for $(\mathrm{GEN}=1 ; \mathrm{GEN}<100 ; \mathrm{GEN}++)$

l

//PH,PC and PT are current morph proportions

//Compute these from the genotypes

$\mathrm{Ph}=\mathrm{Phh}$;

$\mathrm{Pc}=\mathrm{Phc}+\mathrm{Pcc}$;

$\mathrm{Pt}=\mathrm{Pht}+\mathrm{Pct}+\mathrm{Ptt}$;

solve_MF(\&PH,\&PC,\&PT,Ph,Pc,Pt);

${ }^{*}$ calculate probabilities of mating with each genotype */

* Ist mating */

$\mathrm{M} 1 \mathrm{hh}=\mathrm{Phh} * \mathrm{PH}$;

$\mathrm{Mlhc}=\mathrm{Phc} * \mathrm{PC} ;$

$\mathrm{Mlcc}=\mathrm{Pcc} * \mathrm{PC}$;

$\mathrm{Mlht}=\mathrm{Pht} * \mathrm{PT}$

Mlct $=$ Pct $*$ PT;

$\mathrm{M} 1 \mathrm{tt}=\mathrm{Ptt} * \mathrm{PT}$

\section{/* Normalise */}

Normalise(\&Mlhh,\&Mlhc,\&Mlcc,\&Mlht,\&Mlct,\&Mltt);

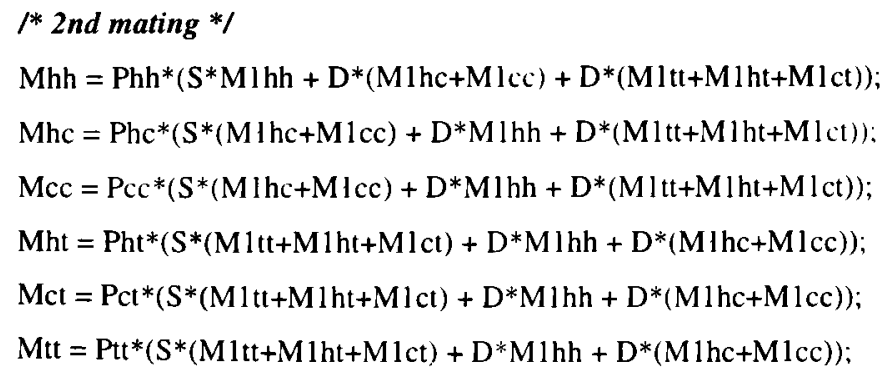

\section{/* Normalise */}

Normalise(\&Mhh,\&Mhc,\&Mcc,\&Mht,\&Mct,\&Mtt); 


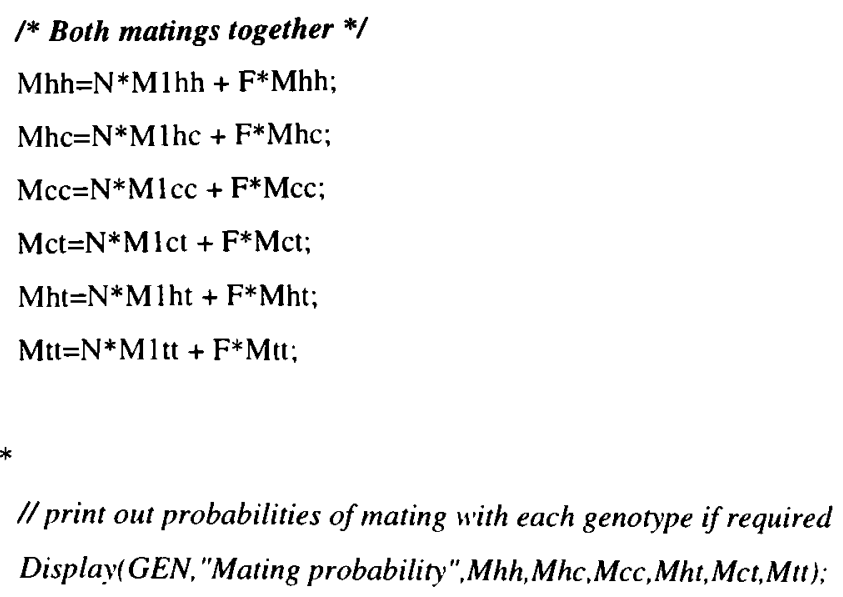

\section{/*Normalise */}

Normalise(\&lhh,\&Ihc,\&Icc,\&Iht,\&lct,\&Itt);

\section{/*PREDATION */}

/*

// Sigmoidal predation: not used

$P h h=I h h * \operatorname{sigmoid}(I h h / 2, a, x \min , x \max , y \min , y \max )$;

$P h c=I h c *$ sigmoid $((I h c+I c c) / 2, a, x \min , x \max , y \min , y \max ) ;$

$P c c=I c c *$ sigmoid $((I h c+l c c) / 2, a, x \min , x \max , y \min , y \max )$;

$P h t=I h t^{*}$ sigmoid $((I h t+I c t+I t t) / 2, a, x \min , x \max , y \min , y \max )$;

$P_{c t}=I c t{ }^{*} \operatorname{sigmoid}((I h t+I c t+I t t) / 2, a, x \min , x \max , y \min , y \max )$;

$P t t=I t{ }^{*} \operatorname{sigmoid}((l h t+I c t+l t t) / 2, a, x \min , x \max , y \min , y \max ) ;$

*/

/* predation function for Batesian mimics - Huheey's function used*/

AmodelH=RmodelH ${ }^{*} \mathrm{CH} /(\mathrm{Ihh})$;

AmodelC $=\mathrm{RmodelC}^{*} \mathrm{CC} /(\mathrm{Ihc}+\mathrm{Icc})$;

Amodel T=RmodelT $* \mathrm{CT} /(\mathrm{Iht}+\mathrm{Ict}+\mathrm{Itt})$;

$\mathrm{Phh}=\mathrm{Ihh}{ }^{*}\left((1-\right.$ Pencounter $)+$ Pencounter ${ }^{*}$ predation(AmodelH, I-AmodelH,N_MEM)); 
Phc $=$ Ihc* $((1-$ Pencounter $)+$ Pencounter* predation(AmodelC,1-AmodelC,N_MEM));

$P c c=I c{ }^{*}\left((1-P e n c o u n t e r)+\right.$ Pencounter ${ }^{*}$ predation(AmodelC,1-AmodelC,N_MEM));

$\mathrm{Pht}=\mathrm{Iht}^{*}((1-$ Pencounter $)+$ Pencounter*predation(AmodelT,1-AmodelT,N_MEM $\left.)\right)$;

Pct $=$ Ict $^{*}\left((1-\right.$ Pencounter $)+$ Pencounter ${ }^{*}$ predation $($ AmodelT,1-AmodelT,N_MEM));

$P t t=I t{ }^{*}((1-$ Pencounter $)+$ Pencounter*predation(AmodelT, I-AmodelT,N_MEM));

\section{/* predation of males */}

Phh+=Ihh* ((1-Pencounter $)+$ Pencounter*predation(0,1,N_MEM));

Phc+=Ihc* ((1-Pencounter $)+$ Pencounter* ${ }^{*}$ redation $(0,1$, N_MEM $\left.)\right)$;

Pcc+=Icc* ((1-Pencounter $)+$ Pencounter*predation $(0,1$, N_MEM $))$;

Pht+=Iht*((1-Pencounter $)+$ Pencounter*predation $\left.\left(0,1, N \_M E M\right)\right)$;

Pct+=Ict* $\left((1-\right.$ Pencounter $)+$ Pencounter* ${ }^{*}$ predation $(0,1$, N_MEM $\left.)\right)$;

Ptt+=Itt*((1-Pencounter $)+$ Pencounter*predation $\left.\left(0,1, N \_M E M\right)\right)$;

\section{"Normalise */}

Normalise $(\& \mathrm{Phh}, \& \mathrm{Phc}, \& \mathrm{Pcc}, \& \mathrm{Pht}, \& \mathrm{Pct}, \& \mathrm{Ptt})$;

\section{$I^{*}$ print out frequencies from subsequent generations */}

Display(GEN,"Gene frequencies",Phh,Phc,Pcc,Pht,Pct,Ptt);

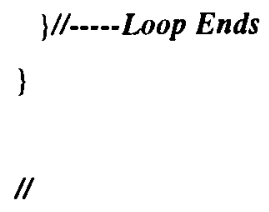


void Display(int GEN, const char *comment, double Phh, double Phc, double Pcc, double Pht, double Pct, double $\mathrm{Ptt})$

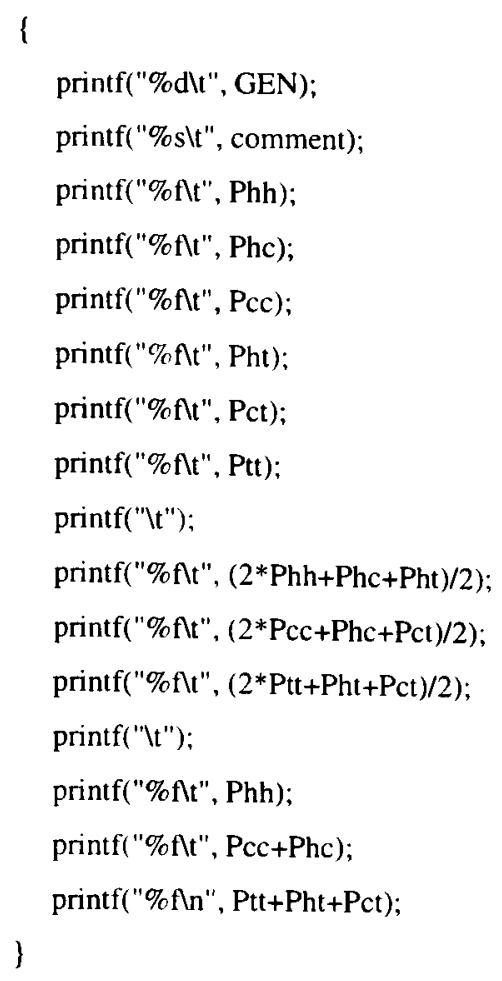

\section{* Subroutine to normalise morph frequencies */} void Normalise $\left(\right.$ double $*$ a, double $*$ b, double ${ }^{*}$ c, double ${ }^{*}$, double ${ }^{*}$, double ${ }^{*}$ f)

\section{/*Batesian mimicry predation function */}

double predation(double Pmodel, double Pmimic, double n_memory)

f

return 1-(1/(n_memory*Pmodel+Pmimic $))$;

\}

\section{* Subroutine to calculate a sigmoidal predation function - not eventually used in this model */} double sigmoid(double $x$ dash, double a, double lxmin, double lxmax, double lymin, double lymax) \{

double fmax, fmin, $\mathbf{x} ; / *$ function for predator selection */ $\mathrm{fmin}=\exp \left(\mathrm{a}^{*} \mathrm{lx \operatorname {min }}\right) /\left(1+\exp \left(\mathrm{a}^{*} \mathrm{l} \operatorname{xmin}\right)\right)$; $f \max =\exp \left(a^{*} \operatorname{lxmax}\right) /\left(1+\exp \left(a^{*} \operatorname{lxmax}\right)\right)$; $\mathrm{x}=\mathrm{xdash} *(\mathrm{x} \max -\mathrm{lx} \min )+\mathrm{lx} \min$; return $\left(1-\left(\operatorname{lymin}+(\operatorname{lymax}-\operatorname{lymin})^{*}\left(\exp \left(\mathrm{a}^{*} \mathrm{x}\right) /\left(1+\exp \left(\mathrm{a}^{*} \mathrm{x}\right)\right)-\mathrm{fmin}\right) /(\mathrm{fmax}-\mathrm{fmin})\right)\right)$; \} 


\section{Appendix 6 Program listing}

void solve_GF(double H,double C,double T, double ${ }^{*} \mathrm{~h}$, double ${ }^{*} \mathrm{c}$, double ${ }^{*} \mathrm{t}$ )

\{

*h=sqrt $(\mathrm{H})$;

${ }^{*} \mathrm{c}=$ solve_quad $(1,2 * * \mathrm{~h},-\mathrm{C})$;

${ }^{*} \mathrm{t}=\operatorname{solve}$ _quad $\left(1,2 *\left({ }^{*} \mathrm{~h}+{ }^{*} \mathrm{c}\right),-\mathrm{T}\right)$;

\}

\section{/* Subroutine to solve morph frequencies from gene frequencies */}

void solve_MF(double ${ }^{*} \mathrm{H}$, double ${ }^{*} \mathrm{C}$, double ${ }^{*} \mathrm{~T}$, double $\mathrm{h}$, double $\mathrm{c}$, double $\mathrm{t}$ ) \{

${ }^{*} \mathrm{H}=\mathrm{h} * \mathrm{~h}$;

${ }^{*} \mathrm{C}=\mathrm{c} * \mathrm{c}+2{ }^{*} \mathrm{~h} * \mathrm{c}$;

$* \mathrm{~T}=\mathrm{t} * \mathrm{t}+2 * \mathrm{~h} * \mathrm{t}+2 * \mathrm{c} * \mathrm{t}$

)

double solve_quad(double a, double b, double c)

\{

//return the positive root of the quadratic

return $\left(\operatorname{sqrt}\left(b^{*} b-4 * a * c\right)-b\right) /(2 * a)$;

\} 